Marcelo Tanaka Hayashi

\title{
Estudo Conceitual do Problema Adjunto Baseado nas EquaÇões de Euler Para APLICAÇõES DE OTIMIZAÇÃo AERODINÂMICA
}

Dissertação apresentada à Escola Politécnica da Universidade de São Paulo para obtenção do Título de Mestre em Engenharia Mecânica. 
Marcelo Tanaka Hayashi

\section{Estudo Conceitual do Problema AdJunto BASEAdo NAS EquAÇÕES DE EULER PARA APLICAÇÕES DE OTIMIZAÇÃO AErodinÂMICA}

Dissertação apresentada à Escola Politécnica da Universidade de São Paulo para obtenção do Título de Mestre em Engenharia Mecânica.

Área de concentração:

Engenharia Mecânica

Orientador:

Prof. Dr. Ernani Vitillo Volpe 
Este exemplar foi revisado e alterado em relação à versão original, sob responsabilidade única do autor e com a anuência de seu orientador.

São Paulo, 5 de Março de 2009

Assinatura do autor

Assinatura do orientador

Ficha Catalográfica

Hayashi, Marcelo Tanaka

Estudo Conceitual do Problema Adjunto Baseado nas Equações de Euler para Aplicações de Otimização Aerodinâmica. São Paulo, 2009. $114 \mathrm{p}$.

Dissertação (Mestrado) - Escola Politécnica da Universidade de São Paulo. Departamento de Engenharia Mecânica.

1. Método Adjunto. 2. Otimização. 3.Aerodinâmica. 4. Dinâmica dos Fluidos Computacional. 5. Condições de Contorno. I. Universidade de São Paulo. Escola Politécnica. Departamento de Engenharia Mecânica. II. Título. 
Aos meus pais, Julio e Nobuco, que nunca pouparam esforços em prol da minha educação.

E à minha irmã, Ana Paula, pelo carinho e incentivo durante todos estes anos de intensa dedicação às atividades de pesquisa. 


\section{AgRADECIMENTOS}

Primeiramente agradeço ao Prof. Dr. Ernani Vitillo Volpe, pela intensa dedicação ao trabalho desenvolvido, pela confiança no meu potencial acadêmico e pela excelente orientação ao me ajudar a formar uma base sólida de conhecimentos em engenharia durante este período de convivência.

Ao Prof. Dr. Luis Carlos de Castro Santos, pelas excelentes sugestões dadas durante a fase de implementação do código computacional, que foram indispensáveis para superar algumas das inúmeras dificuldades enfrentadas no desenvolvimento deste trabalho.

Ao Prof. Dr. João Luiz Filgueiras de Azevedo, por apresentar os fundamentos da Dinâmica dos Fluidos Computacional no curso Métodos Numéricos para Escoamento Compressível I. Os conhecimentos adquiridos na disciplina foram, certamente, imprescindíveis para o estudo do método adjunto e suas condições de contorno.

Aos membros da banca do exame de qualificação, Prof. Titular José Augusto Penteado Aranha e Prof. Livre Docente Fábio Saltara, pelas valiosas sugestões apresentadas para a melhora do texto desta dissertação.

Ao Conselho Nacional de Desenvolvimento Científico e Tecnológico (CNPq) que, através da concessão da bolsa de estudos, propiciou a minha dedicação integral para o desenvolvimento da pesquisa.

Ao Núcleo de Dinâmica dos Fluidos (NDF-EPUSP), particularmente ao Prof. Livre Docente Julio Romano Meneguini, que autorizou a utilização de computadores, sem os quais não seria possível realizar as simulações numéricas contidas neste trabalho.

Meus agradecimentos se estendem ao agora doutorando, Marco Antônio de Barros Ceze, que contribuiu muito para a realização deste trabalho com a sua experiência em otimização aerodinâmica, ao já engenheiro Bruno Galelli Chieregatti, pela dedicação à implementação de parte do código desenvolvido, e aos companheiros de pós-graduação Iago Barbeiro, Alessandro Lima, Herval Tortorelli, Carlos Ilário e Reinaldo Orselli. 
A todos os professores que contribuiram para minha formação nestes anos de mestrado. Em especial ao mestre Andrei Agostinho, Felipe Dias, Josué Agulha, Eduardo Araújo, Rodrigo da Matta, Daniela Amato, Cecile Loyer, Iara Garcia, Andrea Kirst, Mayumi e Mitie.

Por fim, aos meus grandes amigos Bruno Roberti, Thales Albuquerque, Sergio Carneiro, Danilo Micheletto, Marcos Aivazoglou, Ricardo Nakasone, Fabio Carneiro, Vitor Carrara, Roberto Sakaki e Miori Takahashi, presentes em todos os momentos importantes da minha vida. 
“...é impossivel explicar honestamente as belezas contidas nas leis da natureza, de uma forma que as pessoas possam senti-las, sem que elas tenham uma boa compreensão da matemática."

(Richard Feynman) 


\section{RESUMO}

Ao longo da última década o método adjunto tem sido consolidado como uma das mais versáteis e bem sucedidas ferramentas de otimização aerodinâmica e projeto inverso na Dinâmica dos Fluidos Computacional. Ele se tornou uma área de pesquisa por si só, criando uma grande variedade de aplicações e uma literatura prolífica. Entretanto, alguns aspectos relevantes do método permanecem ainda relativamente pouco explorados na literatura, como é o caso das condições de contorno adjuntas e, mais especificamente, com respeito a fronteiras permeáveis. Esta dissertação discute detalhadamente uma nova forma de tratar o problema de contorno, que tem como objetivo assegurar que as equações adjuntas sejam bem-postas.

O principal objetivo da otimização aerodinâmica consiste na tentativa de minimizar (ou maximizar) uma determinada medida de mérito. As aplicações de projeto inverso são desenvolvidas para escoamentos Euler 2-D ao redor de aerofólios, representados com a parametrização CST (Class-Shape function Transformation) proposta por Kulfan e Bussoletti (2006), em regime de vôo transônico e com domínio discretizado por malhas não-estruturadas de triângulos através de um ciclo de projeto, que utiliza o método steepest descent como algoritmo de busca da direção que minimiza (ou maximiza) a função de mérito.

As equações adjuntas são derivadas na sua formulação contínua e suas condições de contorno são determinadas por equações diferenciais características adjuntas e relações de compatibilidade compatíveis com as variações realizáveis da física do escoamento. As variáveis adjuntas são, então, vistas como forças de vínculo generalizadas, que asseguram a realizabilidade de variações do escoamento.

Palavras chave: método adjunto, otimização, aerodinâmica, dinâmica dos fluidos computacional, condições de contorno. 


\section{ABStRaCT}

Over the last decade the adjoint method has been consolidated as one of the most versatile and successful tools of aerodynamic optimization and inverse design in Computational Fluid Dynamics. It has become a research area of its own, spawning a large variety of applications and a prolific literature. Yet, some relevant aspects of the method remain relatively less explored in the literature. Such is the case with the adjoint boundary conditions and, more specifically, with regard to permeable boundaries. This dissertation discusses at length a novel approach to the boundary problem, which aims at ensuring the well-posedness of the adjoint equations.

The main goal of aerodynamic optimization consists in attempting to minimize (or maximize) a certain mesure of merit. The inverse design applications are developed for 2-D Euler flows around airfoils, represented with the CST (ClassShape function Transformation) parameterization proposed by Kulfan and Bussoletti (2006), in the transonic flight regime and domain discretized by triangle unstructured meshes in a design loop which makes use of the steepest descent method as search direction that minimizes (or maximizes) the mesure of merit.

Adjoint equations are derived in the continuous formulation and their boundary conditions are determined by adjoint characteristic differential equations and compatibility relations. The latter are derived so as to be compatible with the realizable variations of physical quantities. The adjoint variables are seen as generalized constraint forces, which ensure the realizability of flow variations.

Key words: adjoint method, optimization, aerodynamics, computational fluid dynamics, boundary conditions. 


\section{SUMÁRIO}

\section{Lista de Figuras}

Lista de Tabelas

Acrônimos

Nomenclatura

1 Introdução 1

1.1 Cenário Atual e Escopo do Trabalho . . . . . . . . . . . . . . . 4

1.2 Diferenças Finitas Versus Adjunto. . . . . . . . . . . 6

1.3 Ciclo de Projeto . . . . . . . . . . . . . . . . . . 10

2 Modelo Matemático do Escoamento 12

2.1 Formulação Característica das Equações de Euler . . . . . . . . . 15

2.2 Adimensionalização das Equações de Euler . . . . . . . . . . . . . 20

2.3 Método Numérico . . . . . . . . . . . . . . . . . . 23

2.3.1 Definição dos Volumes de Controle . . . . . . . . . . . 24

2.3.2 Discretização das Equações de Euler . . . . . . . . . . . 26

2.3.3 Avaliação de Derivadas Espaciais . . . . . . . . . . . 27

2.3.4 Dissipação Artificial . . . . . . . . . . . . . . . 28

2.3.5 Esquema de Marcha no Tempo . . . . . . . . . . . 32

2.4 Condições de Contorno . . . . . . . . . . . . . . . . . . . 34

2.4 .1 Os Volumes "Fantasmas" . . . . . . . . . . . . . . . . 37

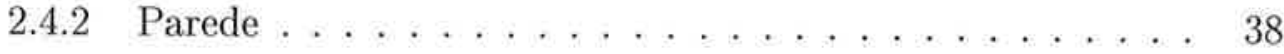

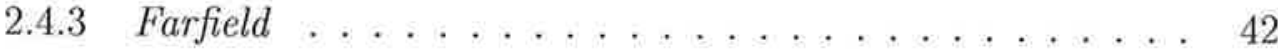


3 Teoria de Controle Aplicada às Equações de Euler

3.1 As Equações Adjuntas . . . . . . . . . . . . . . . . . 56

3.1.1 Redução do Gradiente . . . . . . . . . . . . . . . . 61

3.2 Método Numérico . . . . . . . . . . . . . . . . . . 64

3.2 .1 Dissipação Artificial . . . . . . . . . . . . 65

3.2 .2 Esquema de Marcha no Tempo ............ 66

4 Condições de Contorno do Problema Adjunto $\quad 67$

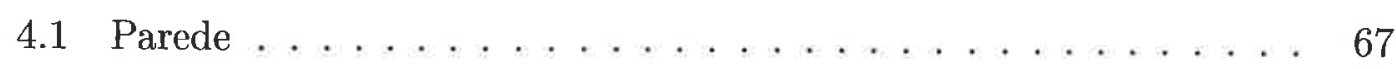

4.2 Farfield ............................... 69

4.2 .1 Entrada ...................... 72

4.2 .2 Saída. . . . . . . . . . . . . . . . . 78

5 Parametrização da Geometria $\quad 82$

5.1 Descrição Matemática da Geometria do Aerofólio . . . . . . . . . 82

5.2 Propriedades da Parametrização CST . . . . . . . . . . . . 83

6 Resultados e Discussões $\quad 86$

6.1 Casos de Validação . . . . . . . . . . . . . . . . . 87

6.2 Casos de Aplicação . . . . . . . . . . . . . . . . . . . . 92

6.3 Sentido de Propagação das Perturbações . . . . . . . . . . . . . . 94

7 Conclusões e Trabalhos Futuros $\quad 97$

7.1 Conclusões . . . . . . . . . . . . . . . . . 97

7.2 Trabalhos Futuros . . . . . . . . . . . . . . . . . . . 98

$\begin{array}{ll}\text { Referências } & 100\end{array}$

Apêndice A - As Equações de Euler em Coordenadas Generalizadas 104 
Apêndice B - Uma Propriedade das Equações de Euler em Coordenadas Generalizadas

Apêndice C - Definição dos Coeficientes na Formulação Característica das Equações Adjuntas 


\section{LisTA DE FiguRAS}

1.1 Ciclo de projeto . . . . . . . . . . . . . 10

2.1 Comparação dos esquemas de discretização . . . . . . . . . . . 25

2.2 Análise de Fourier de um choque unidimensional . . . . . . . . 28

2.3 Efeito da adição de dissipação artificial no espectro de onda . . . 30

2.4 Vetores normais nas fronteiras do domínio . . . . . . . . . . 36

2.5 Exemplo de construção de volumes "fantasmas" . . . . . . . . . 37

2.6 Propagação de características em uma parede sólida . . . . . . . 38

2.7 Propagação de características em fronteiras de entrada . . . . . 44

2.8 Propagação de características em fronteiras de saída . . . . . . . 47

2.9 Caso 1 - Perfil NACA $0012\left(M_{\infty}=0.3, \alpha=0.0^{\circ}\right) \ldots \ldots$

2.10 Caso $2-$ Perfil RAE $2822\left(M_{\infty}=0.3, \alpha=0.0^{\circ}\right) \ldots \ldots$

2.11 Caso 3 - Perfil Selig $1223\left(M_{\infty}=0.1, \alpha=8.0^{\circ}\right) \ldots \ldots 1$

2.12 Caso 4 - Perfil NACA $0012\left(M_{\infty}=0.8, \alpha=0.0^{\circ}\right) \ldots \ldots 2$

2.13 Caso 5 - Perfil RAE $2822\left(M_{\infty}=0.8, \alpha=0.0^{\circ}\right) \ldots \ldots 52$

2.14 Caso 6 - Perfil RAE $2822\left(M_{\infty}=0.75, \alpha=1.0^{\circ}\right) \ldots 53$

2.15 Campo de solução do Caso $6\left(M_{\infty}=0.75, \alpha=1.0^{\circ}\right) \ldots 53$

2.16 Malha utilizada . . . . . . . . . . . . . . . 54

4.1 Propagação de características adjuntas em fronteiras de entrada . 72

4.2 Propagação de características adjuntas em fronteiras de saída . . . 79

5.1 Comparação da parametrização com diferentes graus de aproximação 84

5.2 Erro da parametrização com relação ao número de parâmetros . . 85

6.1 Caso de validação 1 - RAE 2822 para NACA $0012 \ldots 88$

6.2 Campo de solução adjunta do caso de validação 1 . . . . . . . 89 
6.3 Solução adjunta do caso de validação 1 . . . . . . . . . . . . . . 90

6.4 Caso de validação 2 - NACA 0012 para RAE 2822 . . . . . . . . 91

6.5 Caso de aplicação $1 \ldots \ldots$. . . . . . . . . . . . . . . . . 93

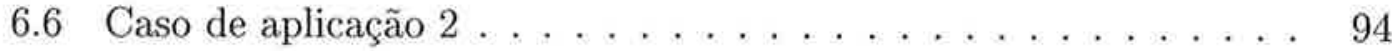

6.7 Propagação de perturbações do escoamento . . . . . . . . . . . . 95

6.8 Propagação de perturbações da solução adjunta . . . . . . . . . . 96

A.1 Transformação de coordenadas . . . . . . . . . . . . . . . . . . 104 


\section{LISTA DE TABELAS}

2.1 Condições de contorno físicas e numéricas para escoamentos 2-D . 43

2.2 Casos de validação do solver do escoamento . . . . . . . . . . . . 49

4.1 Resumo das condições de contorno adjuntas de entrada . . . . . 78

4.2 Resumo das condições de contorno adjuntas de saída . . . . . . . 81

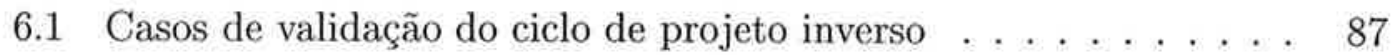

6.2 Casos de aplicação do ciclo de projeto inverso . . . . . . . . . . 92

C.1 Coeficientes da formulação característica das equações adjuntas . 114 


\section{ACRÔNIMOS}

CFD Computational Fluid Dynamics

CFL Número de Courant-Friedrichs-Lewy

CNPq Conselho Nacional de Desenvolvimento Científico e Tecnológico

CST Class-Shape function Transformation

EMBRAER Empresa Brasileira de Aeronáutica

ESSS Engineering Simulation and Scientific Software

FAPESP Fundação de Amparo à Pesquisa do Estado de São Paulo

MGM Modified Garabedian-McFadden

NACA National Advisory Committee for Aeronautics

NDF Núcleo de Dinâmica dos Fluidos

ODE Ordinary Differential Equation

PDE Partial Differential Equation 


\section{NOMENCLATURA}

$a_{c} \quad$ Aceleração centrípeta,

$a_{*} \quad$ Velocidade do som crítica,

A Matriz Jacobiana das variáveis conservadas na direção coordenada $x$,

A Matriz Jacobiana das variáveis primitivas na direção coordenada $x$,

$a_{m} \quad$ Amplitude de onda da $m$-ésima componente,

$\mathbf{A}_{n} \quad$ Matriz Jacobiana associada à uma direção $\vec{k}$,

B Matriz Jacobiana das variáveis conservadas na direção coordenada $y$,

B Matriz Jacobiana das variáveis primitivas na direção coordenada $y$,

C Função de classe,

$C$ ( ) Operador convectivo,

$\bar{c} \quad$ Corda do aerofólio,

$C_{p} \quad$ Calor específico à pressão constante,

c Velocidade do som,

$C_{v} \quad$ Calor específico à volume constante,

$D$ ( ) Operador dissipação artificial,

$d^{(2)}($ ) Operador Laplaciano não-dividido,

$d^{(4)}()$ Operador bi-harmônico,

D Domínio,

E Vetor de fluxos na direção $x$,

e Energia específica,

$\overline{\mathbf{E}} \quad$ Vetor de fluxos na direção $\xi$, 
$e_{i} \quad$ Energia interna específica,

F Vetor de fluxos na direção $y$,

$\overline{\mathbf{F}} \quad$ Vetor de fluxos na direção $\eta$,

$\mathcal{G}$ Gradiente de sensibilidade,

$H \quad$ Entalpia total,

I Medida de mérito,

$\hat{\imath}$ Versor da coordenada cartesiana $x$,

$\hat{\jmath}$ Versor da coordenada cartesiana $y$,

$\vec{k} \quad$ Vetor genérico,

$\hat{k}$ Versor da coordenada cartesiana $z$,

$k_{x} \quad$ Componente cartesiana do vetor $\vec{k}$ na direção $x$,

$k_{y} \quad$ Componente cartesiana do vetor $\vec{k}$ na direção $y$,

$\ell \quad$ Unidade de comprimento,

$\ell_{0} \quad$ Comprimento característico,

$m \quad$ Número de onda,

M Matriz de transformação das variáveis conservadas para primitivas,

$\vec{n} \quad$ Versor normal apontando para fora do volume de controle,

$p \quad$ Pressão estática,

$p_{d} \quad$ Pressão desejada,

$\overrightarrow{\mathcal{P}} \quad$ Vetor de fluxo "vetorial",

Q Vetor de variáveis conservadas,

$R$ Constante do gás,

$R_{L E}$ Raio de curvatura do bordo de ataque,

$R_{w} \quad$ Raio de curvatura local,

S Função de forma, 
$\mathcal{S} \quad$ Área da superfície do volume $\mathcal{V}$,

$T$ Temperatura,

$u$ Componente $x$ do vetor velocidade,

$U$ Módulo do vetor velocidade,

$v \quad$ Componente $y$ do vetor velocidade,

$\overrightarrow{\mathbf{v}} \quad$ Vetor velocidade,

$v_{n} \quad$ Componente normal do vetor velocidade,

$\mathcal{V} \quad$ Volume do domínio computacional,

$\mathcal{V}_{i} \quad$ Volume do $i$-ésimo elemento do domínio,

V Vetor de variáveis primitivas,

$v_{t} \quad$ Componente tangencial do vetor velocidade,

W Vetor de variáveis características,

\section{Subscritos}

$\infty$ Propriedade do escoamento não perturbado,

\section{Símbolos Gregos}

$\alpha \quad$ Angulo de ataque,

$\beta$ Ângulo de fechamento do bordo de fuga,

$\triangle y_{T E}$ Espessura do bordo de fuga,

$\gamma \quad$ Razão de calores específicos,

$\Lambda \quad$ Matriz diagonal,

$\lambda$ Passo do processo de otimização,

$\nabla \quad$ Operador divergente,

$\phi \quad$ Uma propriedade qualquer,

$\Psi \quad$ Vetor de variáveis adjuntas,

$\rho$ Massa específica, 
$\sigma(\vec{k}) \quad$ Autovalor da matriz $\mathbf{A}_{n}$,

Sobrescritos

* Propriedade adimensional,

T Matriz transposta, 


\section{INTRODUÇÃO}

De modo a compreender melhor os fenômenos físicos da natureza, o homem, ao longo de sua existência, sempre procurou desenvolver modelos matemáticos para descrevê-los em diversos níveis de aproximação. O movimento de fluidos, em particular, é completamente descrito pelas leis de conservação de três propriedades básicas: massa, quantidade de movimento e energia (HIRSCH, 1988a).

Estas leis de conservação são representadas matematicamente por um sistema de equações diferenciais à derivadas parciais ( $\mathrm{PDE}^{1}$ 's) não-lineares para os quais não são conhecidas soluções analíticas gerais, mas, apenas, de uma pequena coleção de problemas com interesse prático limitado. Assim, o único modo de analisar o escoamento sobre configurações complexas, com confiança, sempre foi a experimentação em laboratórios. Entretanto, ela tem um custo muito elevado e, nem sempre é possível realizá- la pela dificuldade de reprodução das condições reais como, por exemplo, em escoamentos supersônicos à grandes altitudes (MALISKA, 2004).

Assim, com o advento do computador, houve um grande esforço no sentido de desenvolver métodos numéricos para resolver este tipo de problema, o que resultou na criação de uma nova área da Mecânica dos Fluidos conhecida por Dinâmica dos Fluidos Computacional $\left(\mathrm{CFD}^{2}\right)$. Inicialmente, devido a grandes limitações de processamento das máquinas e, também, dos métodos numéricos conhecidos, era possível resolver apenas equações que modelavam fenômenos físicos de forma bastante simplificada. Contudo, é importante destacar que, apesar de sua simplicidade, métodos como o dos painéis foram muito úteis na indústria aeronáutica.

As incontáveis possibilidades de aplicações que a CFD propiciava levou à intensas investigações realizadas pela academia e que resultaram em métodos numéricos mais robustos e confiáveis. Este fato aliado ao vertiginoso crescimento dos recursos computacionais, tanto no que diz respeito à velocidade de processamento

\footnotetext{
${ }^{1}$ sigla em inglês para Partial Differential Equation.

${ }^{2}$ sigla em inglês para Computational Fluid Dynamics.
} 
quanto na capacidade de armazenamento, permitiram resolver numericamente as equações que governam o escoamento em sua forma mais completa, com níveis de precisão razoáveis e em períodos de tempo plausíveis, tornando a CFD uma ferramenta ainda mais importante na indústria aeronáutica, já que possibilitava a análise de configurações alternativas a custos significativamente mais baixos do que ensaios em túneis de vento, desempenhando, assim, função complementar a estes (GILES, 1997; JAMESON, 1997; MACCORMACK, 1993; SANTOS, 1993).

Apesar de reduzir os custos no projeto de aeronaves, a CFD era utilizada apenas como ferramenta de análise e a estratégia geralmente adotada no projeto destas continuava a ser a tentativa e erro, em que se propõe uma geometria e analisam-se os resultados das simulações, de maneira a obter o efeito desejado. Todavia, essa abordagem depende em larga margem da experiência acumulada do projetista e suas chances de sucesso diminuem sensivelmente à medida que se impõem condições mais elaboradas ao projeto.

Isto ocorre porque não há como garantir que um método de tentativa e erro explore adequadamente o espaço de soluções viáveis. Santos (1995) apresenta uma caracterização geral dos métodos de projeto inverso e otimização, que podem ter um impacto muito positivo nessa situação. Combinados com recursos de CFD, eles permitem uma exploração mais eficiente do espaço de soluções de projeto.

A utilização de técnicas numéricas para otimização de perfis aerodinâmicos em escoamentos transônicos foi iniciada por Hicks, Murman e Vanderplaats (1974) e posteriormente extendida para o projeto de asas por Hicks e Henne (1978). Desde então, diversos métodos de otimização aerodinâmica foram desenvolvidos, dentre eles o método Modified Garabedian-McFadden (MGM) baseado no modelo potencial linearizado do escoamento (SANTOS, 1993; VOLPE, 2004; VOLPE, 2005; VOLPE et al., 2007) e o método adjunto (JAMESON, 1988; REUTHER, 1996; SANTOS, 1995; JAMESON; NADARAJAH, 2000; JAMESON; SRIRAM; MARTINELLI, 2003; JAMESON; KIM, 2003a), tema desta dissertação.

Os métodos de projeto inverso comparam a distribuição de uma quantidade física de interesse a uma distribuição desejada, especificada previamente pelo usuário como o coeficiente de pressão, por exemplo. Com base nessa comparação, esses métodos estimam alterações da geometria que produziriam o efeito desejado. Desta forma é possível, dentre outras coisas, evitar gradientes de pressão adversos que induziriam à separação prematura da camada limite em escoamentos ao redor de aerofólios.

Em geral, não há qualquer garantia de que a distribuição desejada seja factí- 
vel, ou que implique em melhores coeficientes aerodinâmicos, uma vez realizada. Além disso, não se pode garantir que a configuração obtida represente uma solução ótima para as condições prescritas. Assim, a experiência acumulada mantém sua relevância, apenas num nível diferente. Não mais se procura especificar uma geometria que permita alcançar efeitos desejáveis, mas uma distribuição objetivo que corresponda a tais efeitos.

Os métodos de otimização, por outro lado, proporcionam uma abordagem mais abrangente do projeto aerodinâmico. Essencialmente, esses métodos buscam extremos de funções de mérito previamente definidas, que representam pontos de máximo ou mínimo locais no espaço de projeto. De qualquer modo, a existência de um extremo, ainda que local, deve corresponder a uma configuração ótima com respeito à particular medida de mérito adotada.

Como já dito, em problemas inversos existe a possibilidade de que a distribuição de pressão desejada não seja realizável ${ }^{3}$. Esta dificuldade pode ser contornada tratando o problema como um caso particular de um problema de otimização, com uma função de mérito que mede o erro na solução do problema inverso. Desta forma, o processo deve levar até a solução realizável mais próxima do desejado, no sentido dos mínimos quadrados. Assim, converte-se um problema possivelmente mal-posto em um problema bem-posto (JAMESON, 2003). Por exemplo, se $p_{d}$ é a pressão de superfície desejada, pode-se tomar a função de mérito $I$ como sendo a integral:

$$
I=\frac{1}{2} \int_{B_{w}}\left(p-p_{d}\right)^{2} \mathrm{~d} s
$$

É claro que as funções de mérito permitem definições bastante variadas. Além da medida do erro com respeito a uma distribuição de pressões desejada, ainda podem representar integrais de sustentação, arrasto, ou até mesmo a razão da sustentação pelo arrasto. A título de ilustração, a equação (1.2) abaixo mostra uma medida de mérito, representada por uma integral de arrasto, utilizada por Reuther (1996).

$$
I=C_{d}=\frac{1}{\frac{1}{2} \gamma p_{\infty} M_{\infty}^{2} \bar{c}} \oint_{B_{w}} p \frac{\partial y}{\partial \xi} \mathrm{d} \xi
$$

onde $\gamma$ é a razão de calores específicos, $p_{\infty}$ e $M_{\infty}$ são, respectivamente, a pressão e o número de Mach do escoamento não-perturbado, $\bar{c}$ é a corda do aerofólio $B_{w}$ e $\xi$ é a coordenada generalizada tangente à superfície do mesmo (apêndice B).

Há ainda a questão da realizabilidade. Em princípio, ela implica que se imponham restrições ao problema que a garantam. Em aplicações de aerodinâmica,

\footnotetext{
${ }^{3}$ entenda-se por realizável uma distribuição de pressão que satisfaça as equações que governam a física do escoamento.
} 
as condições de realizabilidade são as equações que governam o escoamento. Em última análise, a imposição destas como restrições ao problema de otimização depende da forma como o problema é equacionado.

A teoria de controle de sistemas governados por equações diferenciais (LIONS, 1971) proporciona os fundamentos conceituais e o formalismo necessários a essa atividade. O método inicialmente proposto por Pironneau (1983) para problemas elípticos e, mais tarde extendido para escoamentos transônicos por Jameson (1988) ficou conhecido como método adjunto e faz uso desses recursos com dupla vantagem: de um lạdo, ao impor as equações da mecânica dos fluidos como restrições ao problema variacional, projetam-se as variações da medida de mérito no espaço das soluções realizáveis; de outro, essas restrições permitem uma simplificação excepcional no cálculo do gradiente de sensibilidade, com a conseqüente redução do seu custo computacional.

\subsection{Cenário Atual e Escopo do Trabalho}

O Núcleo de Dinâmica e Fluidos (NDF-EPUSP) iniciou investigação nessa

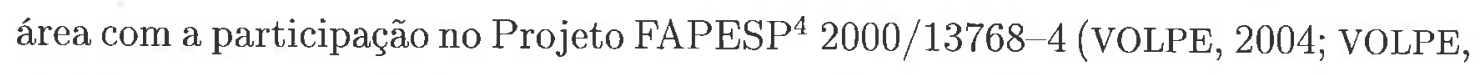
2005). Trabalhando juntamente com pesquisadores da EMBRAER ${ }^{5}$ e da ESSS ${ }^{6}$, desenvolveu-se uma rotina de projeto inverso aerodinâmico baseada no método MGM (VOLPE et al., 2007), que se aplica ao regime de escoamento transônico e tem como finalidade obter uma geometria que aproxime a distribuição de pressões desejada, especificada a priori.

Esta dissertação, juntamente com os trabalhos de Ceze (2008) e Chieregatti (2008), têm como objetivo ampliar essa investigação e consolidar no NDF uma linha de pesquisa na área de otimização e projeto inverso aerodinâmico ao realizar um estudo teórico aprofundado do método adjunto.

A eficiência deste método, naturalmente, instigou o interesse da indústria a utilizá-lo na fase de concepção de aeronaves (JAMESON, 1997), onde, ainda, predominam métodos de projeto inverso associados a modelos específicos do escoamento como o potencial compressível linearizado (NAIK et al., 1995; OLIVEIRA; TRAPP; MACEDO, 2003; SADRI; LEBLOND; PIPERNI, 2002). Entretanto, trabalhos propostos nas áreas de interferência aerodinâmica e integração propulsiva (FUJITA, 2002; MAKINO; IWAMTYA; LEI, 2003; RODRIGUEZ, 2003; KIM; KOC; NA-

\footnotetext{
${ }^{4}$ Fundação de Amparo à Pesquisa do Estado de São Paulo

${ }^{5}$ Empresa Brasileira de Aeronáutica

${ }^{6}$ Engineering Simulation and Scientific Software, Ltda.-- SC, Brasil
} 
KAHASHI, 2005) revelam uma tendência pela adoção do método adjunto (VOLPE, 2005). Todavia, no Brasil, devido a escassez de recursos, ainda não se atingiu massa crítica suficiente nas pesquisas nesta área para que sua implementação no setor aeroespacial brasileiro seja viável.

Afim de viabilizá-la em um futuro próximo, propõe-se iniciar um estudo do método através de uma aplicação restrita e relativamente simples: a otimização de aerofólios imersos em escoamentos modelados pelas equações de Euler bidimensionais que, apesar de representarem uma simplificação da física que governa o movimento dos fluidos, retêm a não-linearidade do termo convectivo, que tem fundamental importância no problema de contorno, inclusive quando se modelam escoamentos viscosos. Para tanto, o domínio será discretizado em malhas não-estruturadas de triângulos, com as propriedades do escoamento atribuídas ao centróide dos elementos, e as equações diferenciais serão resolvidas numericamente com a utilização do método dos volumes finitos em um esquema centrado com adição de dissipação artificial.

Vale acrescentar que o emprego de uma formulação bidimensional do método adjunto em aplicações tridimensionais, via seções de controle, é uma alternativa à complexidade de uma formulação inteiramente tridimensional. Embora esta última seja, certamente, mais fiel à física do escoamento, a primeira pode ser empregada com sucesso em aplicações de interferência aerodinâmica, conforme reportam Kim, Koc e Nakahashi (2005). Enfim, além de ser bastante apropriada para uma exploração introdutória do tema, ela permite aplicações práticas de interesse imediato na indústria.

O escopo deste trabalho, em particular, é estender o procedimento de obtenção de condições de contorno adjuntas proposto por Volpe e Santos (2009) em escoamentos unidimensionais para problemas multidimensionais. É importante notar que, aqui, não há nenhuma pretensão em esgotar as possibilidades de estudo do método adjunto, mas, apenas, contribuir com o tratamento de suas condições de contorno, que são pouco exploradas na literatura. Para isso, propõem-se "construir" o problema adjunto à imagem e semelhança das equações de Euler 2-D, com ambos os problemas de contorno baseados em relações diferenciais características e variáveis de Riemann, garantindo a compatibilidade entre os problemas primal e dual. 


\subsection{Diferenças Finitas Versus Adjunto}

Como já dito anteriormente, medidas de mérito $(I)$ relevantes às aplicações aerodinâmicas podem representar integrais de arrasto ou de uma medida de erro com respeito a uma distribuição de pressões desejada como mostram as equações (1.1) e (1.2). Portanto, geralmente, estas envolvem funcionais ou funções que dependem das variáveis do escoamento $\mathrm{Q}$ e da geometria de suas fronteiras $\mathcal{F}$ (JAMESON; KIM, 2003b; REUTHER, 1996). Assim,

$$
I=I(\mathbf{Q}, \mathcal{F})
$$

Em geral $\mathcal{F}$ pode ser representada como função das coordenadas $\mathcal{X}$, especificada por um conjunto de parâmetros $\mathcal{B}: \mathcal{F}=\mathcal{F}(\mathcal{X}, \mathcal{B})$. Estes parâmetros podem ser, por exemplo, coeficientes de um conjunto de funções de forma. Variações da geometria $\delta \mathcal{F}$ podem ser produzidas por alterações nos parâmetros $\delta \mathcal{B}$ e, naturalmente, elas implicam em variações no campo do escoamento $\delta \mathbf{Q}$. Assim, pode-se escrever:

$$
\delta I=\frac{\partial I^{T}}{\partial \mathbf{Q}} \delta \mathbf{Q}+\frac{\partial I^{T}}{\partial \mathcal{F}} \delta \mathcal{F}
$$

Em princípio, a sensibilidade da medida de mérito a variações da geometria é medida pelo gradiente:

$$
\frac{\partial I}{\partial \mathcal{B}}=\frac{\partial I^{T}}{\partial \mathbf{Q}} \frac{\partial \mathbf{Q}}{\partial \mathcal{B}}+\frac{\partial I^{T}}{\partial \mathcal{F}} \frac{\partial \mathcal{F}}{\partial \mathcal{B}}
$$

Note que os termos $\partial I^{T} / \partial \mathbf{Q}$ e $\partial I^{T} / \partial \mathcal{F}$ são facilmente obtidos, dado que se conhece a expressão da função de mérito que se deseja otimizar. Além do mais, para $\mathcal{F}(\mathcal{X}, \mathcal{B})$ conhecida, as derivadas $\partial \mathcal{F} / \partial \mathcal{B}$ podem ser estimadas sem maiores dificuldades. Entretanto, o termo $\partial \mathbf{Q} / \partial \mathcal{B}$ tem avaliação mais complicada. Uma vez que, cm gcral, não se conhece explicitamente a dependência das variáveis do escoamento com respeito aos parâmetros que controlam a geometria. Além do mais, não é possível garantir a realizabilidade das variações no campo do escoamento $\delta \mathrm{Q}$, que não foi imposta à equação (1.5).

A forma mais direta de se atacar o problema seria avaliar a equação (1.5) por diferenças finitas. Em linhas gerais, o procedimento envolveria obter a solução convergida para a geometria original $\mathcal{F}(\mathcal{X}, \mathcal{B})$, que seria a solução base. Em seguida, cada um dos parâmetros de $\mathcal{F}$ seria perturbado individualmente, $\mathcal{B}_{i}+\delta \mathcal{B}_{i}$, enquanto os demais manteriam seus valores originais. Para cada uma dessas perturbações, seria obtida a solução convergida correspondente. Os valores da 
medida de mérito seriam calculados para as soluções perturbadas e a solução base. De posse desses resultados, seria possível estimar numericamente o gradiente de sensibilidade com relação a cada um dos parâmetros que definem a geometria através da seguinte expressão:

$$
\frac{\partial I}{\partial \mathcal{B}_{i}} \cong \frac{I\left(\mathcal{B}_{i}+\delta \mathcal{B}_{i}\right)-I\left(\mathcal{B}_{i}\right)}{\delta \mathcal{B}_{i}}
$$

Então, o vetor gradiente $\partial I / \partial \mathcal{B}$, que tem sua precisão limitada pela magnitude da variação de cada um dos parâmetros $\delta \mathcal{B}_{i}$, pode ser utilizado para determinar a direção de otimização. Para tanto, é possível utilizar, por exemplo, o método steepest descent que aplica um passo fixo $\lambda$ no sentido negativo do gradiente, obtendo os novos parâmetros otimizados $\mathcal{B}_{i}$ da seguinte maneira:

$$
\mathcal{B}_{i}^{n+1}=\mathcal{B}_{i}^{n}-\lambda \frac{\partial I}{\partial \mathcal{B}_{i}}
$$

Este procedimento assegura a realizabilidade das variações $\delta \mathrm{Q}$ à medida que busca uma solução convergida do escoamento para cada uma das perturbações. Entretanto, ele o faz a custa de grande aumento do esforço computacional. Justamente porque requer uma solução convergida para cada perturbação $\delta \mathcal{B}_{i}$. Em resumo, se $\mathcal{F}$ tem $N$ parâmetros, então são necessárias $N+1$ soluções independentes das equações do movimento, levando-se em conta a solução base (JAMESON; NADARAJAH, 2000), para conseguir obter o gradiente de sensibilidade da medida de mérito com relação aos parâmetros que descrevem a geometria da superfície aerodinâmica que se quer otimizar. É evidente que o custo computacional desta abordagem fica proibitivo à medida que aumenta o número de parâmetros que controlam $\mathcal{F}(\mathcal{X}, \mathcal{B})$.

À princípio, uma forma de contornar esta dificuldade seria restringir, a priori, as variações das grandezas físicas $\delta \mathbf{Q}$ ao espaço das soluções realizáveis, então, talvez, fosse possível eliminar a necessidade dos caros cálculos de $\partial \mathbf{Q} / \partial \mathcal{B}_{i}$ para estimar o gradiente de sensibilidade. O método adjunto explora esta possibilidade por meio de conceitos de teoria de controle para alcançar este objetivo. Basicamente, ele impõe as equações que governam o escoamento como restrições ao problema variacional e, com isso, impede soluções não realizáveis. Para tanto, considera-se que a solução dessas equações depende das variáveis do escoamento e da forma de suas fronteiras, em termos gerais:

$$
\mathcal{R}(\mathbf{Q}, \mathcal{F})=0
$$

Assim, $\mathcal{R}(\mathbf{Q}, \mathcal{F})$ representa o modelo matemático do escoamento e pode ser as 
equações de Euler ou Navier-Stokes, por exemplo. Naturalmente, sua variação pode ser obtida de modo análogo à variação da medida de mérito dada pela equação (1.4), desta forma:

$$
\delta \mathcal{R}=\frac{\partial \mathcal{R}}{\partial \mathbf{Q}} \delta \mathbf{Q}+\frac{\partial \mathcal{R}}{\partial \mathcal{F}} \delta \mathcal{F}=0
$$

Introduzindo as equações do movimento como restrições à função de mérito através de um multiplicador de Lagrange, $\Psi$, tem- se:

$$
\delta I=\frac{\partial I^{T}}{\partial \mathbf{Q}} \delta \mathbf{Q}+\frac{\partial I^{T}}{\partial \mathcal{F}} \delta \mathcal{F}-\Psi^{T}\left(\frac{\partial \mathcal{R}}{\partial \mathbf{Q}} \delta \mathbf{Q}+\frac{\partial \mathcal{R}}{\partial \mathcal{F}} \delta \mathcal{F}\right)
$$

Observe que a introdução destas equações não altera a variação $\delta I$, uma vez que $\delta \mathcal{R}=0$, como mostra a equação (1.9). Entretanto, impõe-se que as variações $\delta \mathbf{Q}$ sejam realizáveis. A equação (1.10) ainda pode ser rearranjada de modo conveniente ao agrupar termos da seguinte maneira:

$$
\delta I=\left(\frac{\partial I^{T}}{\partial \mathbf{Q}}-\boldsymbol{\Psi}^{T} \frac{\partial \mathcal{R}}{\partial \mathbf{Q}}\right) \delta \mathbf{Q}+\left(\frac{\partial I^{T}}{\partial \mathcal{F}}-\Psi^{T} \frac{\partial \mathcal{R}}{\partial \mathcal{F}}\right) \delta \mathcal{F}
$$

Note que a primeira parcela envolve variações do campo do escoamento $\delta \mathrm{Q}$, enquanto a segunda, apenas variações da geometria $\delta \mathcal{F}$. Assim, ao "escolher" $\Psi$ de forma a satisfazer a equação adjunta:

$$
\left[\frac{\partial \mathcal{R}}{\partial \mathbf{Q}}\right]^{T} \Psi=\frac{\partial I}{\partial \mathbf{Q}}
$$

A variação da medida de mérito $\delta I$ pode ser calculada simplesmente pela expressão:

$$
\delta I=\left(\frac{\partial I^{T}}{\partial \mathcal{F}}-\Psi^{T} \frac{\partial \mathcal{R}}{\partial \mathcal{F}}\right) \delta \mathcal{F}=\mathcal{G} \delta \mathcal{F}
$$

Portanto, a resolução da equação adjunta (1.12) permite uma simplificação na forma final de $\delta I$, apresentada pela equação (1.13), que é uma conseqüência direta da imposição da realizabilidade de $\delta \mathbf{Q}$ através da equação (1.9). Note também, que a precisão do gradiente obtido pelo método adjunto não depende mais da magnitude de qualquer variação dos parâmetros $\delta \mathcal{B}_{i}$ como ocorria no caso de diferenças finitas. Ao lado disso, a independência da variação $\delta I$ com relação a $\delta \mathbf{Q}$ permite que se calcule o gradiente de sensibilidade com respeito a qualquer número de parâmetros $\mathcal{B}_{i}$, sem a necessidade de simulações adicionais do escoamento. Essa é a essência do método adjunto proposto por Jameson (JAMESON, 1988; JAMESON, 1994) para escoamentos transônicos.

Note que ambos os termos $\partial \mathcal{R} / \partial \mathbf{Q}$ (que define os coeficientes das variáveis 
adjuntas) e $\partial I / \partial \mathbf{Q}$ (termo não-homogêneo) da equação (1.12) são independentes do vetor de variáveis adjuntas $\Psi$, o que faz da equação adjunta linear. É interessante notar que ela seja linear apesar de ser derivada à partir das equações que governam escoamentos que são, geralmente, não-lineares (como no caso das equações de Euler ou Navier-Stokes) e que formulações dos problemas primal e dual são representações equivalentes do mesmo problema linear ${ }^{7}$, como mostram Giles e Pierce (1997b). Além disso, sua estrutura concentra no lado esquerdo os termos que dependem apenas das equações que governam o escoamento, enquanto o termo não-homogêneo, no lado direito, é o único que envolve a particular medida de mérito adotada.

Se, por um lado, a linearidade traz benefícios claros para as aplicações; por outro, a estrutura da equação adjunta tem profundas consequiências na formulação do método. A separação entre os termos que dependem das equações da mecânica dos fluidos e os que envolvem a medida de mérito implica na existência de um operador adjunto associado apenas ao modelo da física do escoamento. Então, em princípio, ao se obter o operador associado ao modelo em questão, pode-se otimizar uma ampla gama de medidas de mérito, pois isto envolveria modificar apenas o termo não-homogêneo, que por sinal, está presente somente na condição de contorno de parede e que, conseqüentemente, consiste em integrais apenas na superfície aerodinâmica que se deseja otimizar como mostram as equações (1.1) e (1.2). Outra conseqüência imediata desta separação é que, em grande medida, o algoritmo para resolver a equação (1.12) depende do particular operador adjunto considerado, e não do termo forçante. Isso permite enorme flexibilidade na definição das medidas de mérito.

Vale acrescentar que as equações adjuntas são obtidas a partir de uma solução estacionária do escoamento. Assim, as variáveis de estado Q são constantes durante o processo de solução do problema dual e, portanto, o termo $\left[\frac{\partial \mathcal{R}}{\partial \mathbf{Q}}\right]^{T}$ nada mais é que uma matriz quadrada $4 \times 4$ constante para cada elemento da malha computacional durante o processo de solução do problema adjunto no caso bidimensional, por exemplo. Conseqüentemente, basta "invertê-la" para resolver o sistema de equações (1.12) e obter a solução adjunta.

Embora restritas a problemas mais simples, as soluções analíticas baseadas na função de Green ilustram perfeitamente as propriedades apontadas acima. Com este enfoque, Giles e Pierce conduziram extensa investigação das propriedades matemáticas das equações adjuntas e as funções de Green a elas associadas (GI-

\footnotetext{
${ }^{7}$ Observe que a equação (1.9) representa uma linearização do problema primal (1.8) e que o problema adjunto (dual) advém dessa linearização.
} 
LES; PIERCE, 1997a; GILES; PIERCE, 1998a; GILES; PIERCE, 2000b). Obtiveram soluções analíticas para problemas selecionados (GILES; PIERCE, 2000a) e identificaram outras aplicações importantes do método adjunto. Exemplos da variedade de tais aplicações são a estimativa de funcionais de arrasto e sustentação (GILES; PIERCE, 1999a; GILES; PIERCE, 1999b), além da análise numérica em CFD (GILES; PIERCE, 1997a; GILES; PIERCE, 1998b).

\subsection{Ciclo de Projeto}

De modo a realizar o processo de otimização de aerofólios, é necessário implementar um ciclo de projeto, apresentado na figura 1.1, que associa os códigos de geração de malha, otimização e de resolução das equações que governam o escoamento e das equações adjuntas.

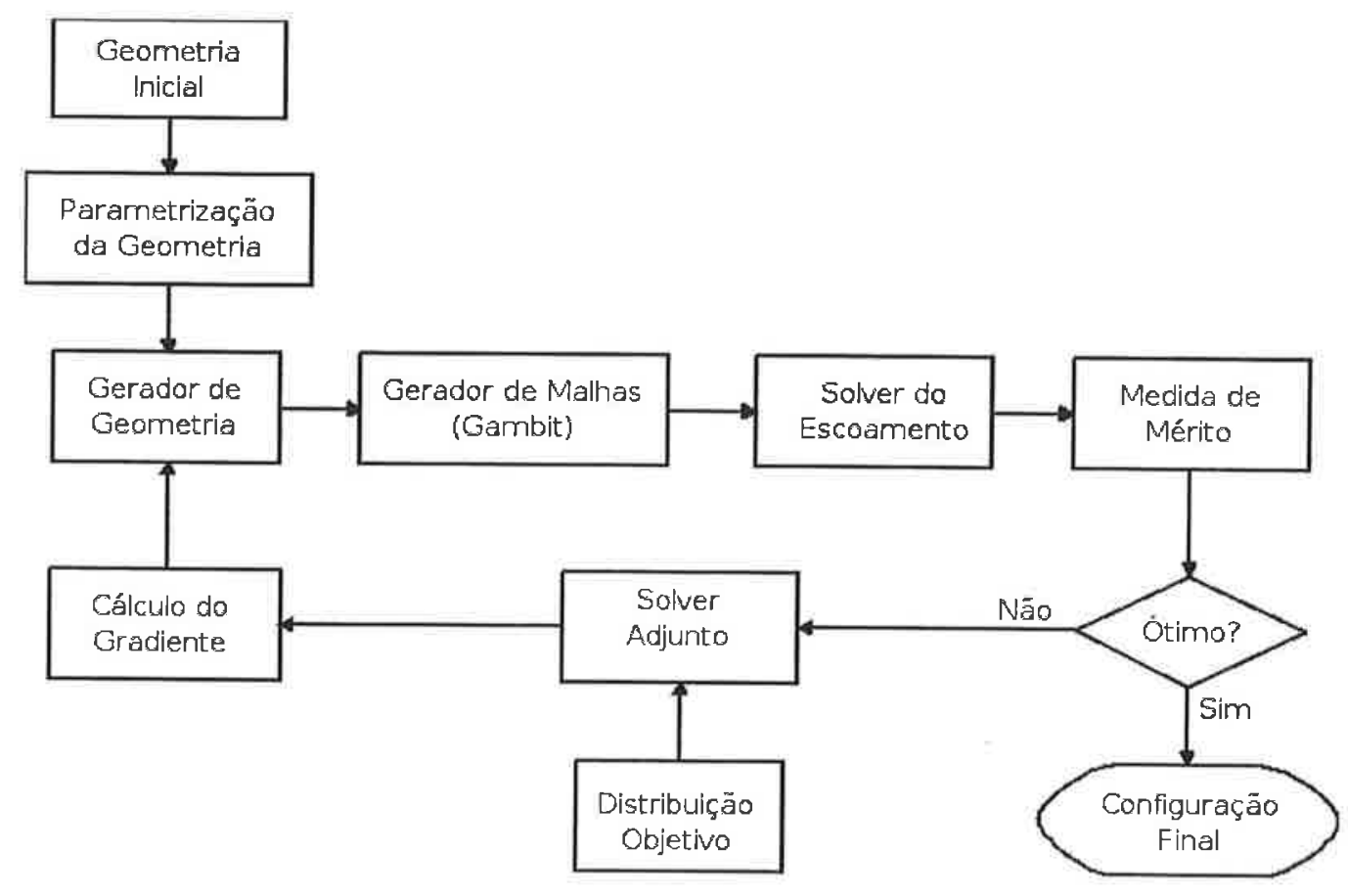

Figura 1.1: Ciclo de projeto

Uma geometria inicial é fornecida como entrada ao ciclo de projeto. Então, é feita uma aproximação da superfície aerodinâmica, a partir da parametrização adotada, através do método de mínimos quadrados. A partir da geometria já parametrizada, gera se uma malha computacional através do programa comercial Gambit, que, por sinal, é a única ferramenta do ciclo de projeto que não foi desenvolvida durante a elaboração deste trabalho. A malha gerada é então enviada ao código que resolve as equações que governam o escoamento. Com a solução 
convergida é feita uma análise da adequação do aerofólio à medida de mérito utilizada, caso o objetivo da otimização não seja atingido (dentro de uma margem de tolerância), resolvem-se as equações adjuntas. Com a solução adjunta, é possível obter o gradiente de sensibilidade da medida de mérito em relação aos parâmetros que descrevem a geometria e, a partir dele, obtém-se os parâmetros que definem a nova geometria utilizando o método steepest descent já descrito anteriormente, que consiste em aplicar um passo fixo na direção negativa do gradiente, fechando assim o ciclo de projeto. Vale mencionar que este método de otimização tem eficiência baixa e que existem algoritmos mais eficientes, entretanto, todos requerem a informação do gradiente. 


\section{Modelo MATEMÁtico DO ESCOAMENTO}

Como já foi dito anteriormente, o movimento dos fluidos é completamente descrito pelas leis de conservação de massa, quantidade de movimento e energia. O modelo matemático que descreve estas leis de conservação com o maior nível de aproximação da física do escoamento é representado pelo sistema de PDE's (2.1) apresentado abaixo em sua forma integral.

$$
\begin{aligned}
\text { massa } & \frac{\partial}{\partial t} \int_{\mathcal{V}} \rho \mathrm{d} \mathcal{V}+\oint_{\mathcal{S}} \rho \overrightarrow{\mathbf{v}} \cdot \mathrm{d} \overrightarrow{\mathcal{S}}=0 \\
\text { quant. mov. } & \frac{\partial}{\partial t} \int_{\mathcal{V}} \rho \overrightarrow{\mathbf{v}} \mathrm{d} \mathcal{V}+\oint_{\mathcal{S}}(\rho \overrightarrow{\mathbf{v}} \cdot \mathrm{d} \overrightarrow{\mathcal{S}}) \overrightarrow{\mathbf{v}}+\oint_{\mathcal{S}} p \mathrm{~d} \overrightarrow{\mathcal{S}}=\oint_{\mathcal{S}} \vec{f}_{e} \mathrm{~d} \mathcal{S} \\
\text { energia } & \frac{\partial}{\partial t} \int_{\mathcal{V}} e \mathrm{~d} \mathcal{V}+\oint_{\mathcal{S}} \rho H \overrightarrow{\mathbf{v}} \cdot \mathrm{d} \overrightarrow{\mathcal{S}}=\oint_{\mathcal{S}} \overrightarrow{f_{e}} \cdot \overrightarrow{\mathbf{v}} \mathrm{d} \mathcal{S}+\oint_{\mathcal{S}} \vec{q} \cdot \mathrm{d} \overrightarrow{\mathcal{S}}
\end{aligned}
$$

Onde $\rho$ é a massa específica de um fluido com velocidade $\overrightarrow{\mathbf{v}}$, sob pressão $p$, sujeito à forças externas $\vec{f}_{e}$, fluxo de calor $\vec{q}$, com entalpia total $H$ e energia específica e em um domínio de volume $\mathcal{V}$ e área de superfície $\mathcal{S}$.

Entretanto, no caso tridimensional, por exemplo, este sistema possui 15 incógnitas (massa específica $\rho, 3$ componentes do vetor velocidade $\overrightarrow{\mathbf{v}}$, pressão estática $p, 6$ componentes do tensor de tensões viscosas $\tau$, energia $e$ e 3 componentes de fluxo de calor) e apenas 5 equações (conservação de massa, 3 componentes da conservação de quantidade de movimento e conservação de energia). Portanto, este sistema de equações é "aberto", uma vez que existem mais incógnitas do que equações. Assim, é necessário complementá-las com um conjunto de relações constitutivas que permitam "fechá-lo". Este sistema de equações completo, incluindo as relações consitutivas, é freqüentemente referenciado na engenharia aeronáutica como "equações de Navier-Stokes", embora este termo tenha sido empregado, originalmente, apenas à equação de conservação da quantidade de movimento, em homenagem à Claude-Louis Navier e George Gabriel Stokes, primeiros a deduzi-la.

As equações de Euler, representadas pelo sistema de equações (2.2) também 
em sua forma integral, são uma simplificação das equações de Navier-Stokes e representam um nível de aproximação da física do escoamento em que as forças viscosas e efeitos de transferência de calor são desprezados. Formalmente, as equações de Euler são obtidas tomando-se o "limite matemático" das equações de Navier-Stokes quando o número de Reynolds tende a infinito (AZEVEDO, 2006), ou seja, quando as forças viscosas são desprezíveis se comparadas às forças inerciais. Apesar de representarem um escoamento idealizado, elas são muito importantes na engenharia aeronáutica, já que grande parte dos escoamentos de interesse na área são de alta velocidade e, com as forças viscosas concentradas numa região limitada do domínio conhecida como camada limite. Além disso, são capazes de capturar efeitos de compressibilidade não-lineares como as ondas de choque.

$$
\begin{aligned}
& \frac{\partial}{\partial t} \int_{\mathcal{V}} \rho \mathrm{d} \mathcal{V}+\oint_{\mathcal{S}} \rho \overrightarrow{\mathbf{v}} \cdot \mathrm{d} \overrightarrow{\mathcal{S}}=0 \\
& \frac{\partial}{\partial t} \int_{\mathcal{V}} \rho \overrightarrow{\mathbf{v}} \mathrm{d} \mathcal{V}+\oint_{\mathcal{S}}(\rho \overrightarrow{\mathbf{v}} \cdot \mathrm{d} \overrightarrow{\mathcal{S}}) \overrightarrow{\mathbf{v}}+\oint_{\mathcal{S}} p \mathrm{~d} \overrightarrow{\mathcal{S}}=0 \\
& \frac{\partial}{\partial t} \int_{\mathcal{V}} e \mathrm{~d} \mathcal{V}+\oint_{\mathcal{S}} \rho H \overrightarrow{\mathbf{v}} \cdot \mathrm{d} \overrightarrow{\mathcal{S}}=0
\end{aligned}
$$

Observe que as equações de Euler tridimensionais também formam um sistema de equações "aberto". Entretanto, apenas uma relação constitutiva é necessária para "fechá-lo", uma vez que existem 6 incógnitas (massa específica $\rho, 3$ componentes do vetor velocidade $\overrightarrow{\mathbf{v}}$, pressão estática $p$, e energia $e$ ) e as mesmas 5 equações.

As equações de Euler podem, no entanto, ser representadas de outras maneiras. A literatura, freqüentemente as apresenta em sua forma conservativa (ou divergente) apresentada no sistema (2.3), que pode ser obtida ao aplicar o teorema de Gauss às integrais de superfície do sistema de equações (2.2), transformandoas em integrais no volume.

$$
\begin{aligned}
& \frac{\partial}{\partial t} \int_{\mathcal{V}} \rho \mathrm{d} \mathcal{V}+\int_{\mathcal{V}} \nabla \cdot(\rho \overrightarrow{\mathbf{v}}) \mathrm{d} \mathcal{V}=0 \\
& \frac{\partial}{\partial t} \int_{\mathcal{V}} \rho \overrightarrow{\mathbf{v}} \mathrm{d} \mathcal{V}+\int_{\mathcal{V}} \nabla \cdot(\rho \overrightarrow{\mathbf{v}} \overrightarrow{\mathbf{v}}) \mathrm{d} \mathcal{V}+\int_{\mathcal{V}} \nabla \cdot p \mathrm{~d} \mathcal{V}=0 \\
& \frac{\partial}{\partial t} \int_{\mathcal{V}} e \mathrm{~d} \mathcal{V}+\int_{\mathcal{V}} \nabla \cdot(\rho H \overrightarrow{\mathbf{v}}) \mathrm{d} \mathcal{V}=0
\end{aligned}
$$

onde o operador divergente $\nabla$, que nomeia esta forma de representação das equações, é dado pela seguinte expressão:

$$
\nabla \equiv \hat{\imath} \frac{\partial}{\partial x}+\hat{\jmath} \frac{\partial}{\partial y}+\hat{k} \frac{\partial}{\partial z}
$$


em que $\hat{\imath}, \hat{\jmath}$ e $\hat{k}$ são os versores nas direções coordenadas $x, y$ e $z$, respectivamente.

Observe, no entanto, que a utilização do teorema de Gauss requer que não haja descontinuidades no volume de controle $\mathcal{V}$ (PRAGER, 1961). Assim, o sistema de equações (2.3), a rigor, não é aplicável em ondas de choque. Nestas regiões, vale apenas a solução fraca, que é obtida somente a partir das equações (2.2) ao considerar a onda de choque isolada em um volume de controle e, então, estabelecer a continuidade dos fluxos de massa, quantidade de movimento e energia na superfície desse volume. Este procedimento equivale à imposição das relações de Rankine Hugoniot (LEVEQUE, 2002).

Uma vez que as equações (2.3) são escritas para um volume arbitrário $\mathcal{V}$, elas devem ser válidas localmente para qualquer ponto do domínio. Isto leva a forma diferencial das leis de conservação (HIRSCH, 2007), apresentada abaixo pela equação (2.5) para escoamentos bidimensionais.

$$
\frac{\partial \mathbf{Q}}{\partial t}+\frac{\partial \mathbf{E}}{\partial x}+\frac{\partial \mathbf{F}}{\partial y}=0
$$

onde o vetor de estados $Q$ e os vetores de fluxo $\mathbf{E}$ e $\mathbf{F}$ são dados por:

$$
\mathbf{Q}=\left\{\begin{array}{c}
\rho \\
\rho u \\
\rho v \\
e
\end{array}\right\} \quad, \quad \mathbf{E}=\left\{\begin{array}{c}
\rho u \\
\rho u^{2}+p \\
\rho u v \\
(e+p) u
\end{array}\right\} \quad, \quad \mathbf{F}=\left\{\begin{array}{c}
\rho v \\
\rho u v \\
\rho v^{2}+p \\
(e+p) v
\end{array}\right\}
$$

De modo a obter a relação constitutiva para completar o sistema de equações é comum, neste tipo de aplicação, utilizar a hipótese de gás termicamente perfeito:

$$
p=\rho R T=(\gamma-1) \rho e_{i}
$$

onde $T$ é a temperatura do fluido, $\gamma$ é a razão de calores específicos, $R$ é a constante do gás e a energia interna específica $e_{i}$ é dada por:

$$
e_{i}=C_{v} T
$$

Como última hipótese, admite-se que o gás seja caloricamente perfeito, ou seja, a relação de calores específicos é constante:

$$
\gamma \equiv \frac{C_{p}}{C_{v}}=\text { constante }
$$

Por fim, a energia específica total, definida pela soma da energia interna com 
a energia cinética, é dada pela seguinte expressão:

$$
e \equiv \rho\left[e_{i}+\frac{1}{2}\left(u^{2}+v^{2}\right)\right]
$$

Associando as equações (2.7) e (2.10), obtém-se a equação de estado (2.11) abaixo que será utilizada para "fechar" o sistema:

$$
p=\left[e-\frac{1}{2}\left(\rho u^{2}+\rho v^{2}\right)\right](\gamma-1)
$$

Observa-se que as equações de Euler são constituídas por um sistema de equações hiperbólicas no domínio espaço-tempo. Portanto, todos os autovalores das matrizes jacobianas associadas aos seus vetores de fluxo são reais (HIRSCH, 1988a). Além disso, a propagação de informação ocorre por meio das características. Estas propriedades são descritas detalhadamente na seção 2.1 e, posteriormente, exploradas sob o aspecto de condições de contorno na seção 2.4

\subsection{Formulação Característica das Equações de Eu- ler}

Considere as equações de Euler bidimensionais em sua forma diferencial conservativa dada pela expressão (2.5). Note que os vetores de fluxo E e F podem ser escritos em função das variáveis conservadas do vetor de estado Q. Então, pela regra da cadeia:

$$
\frac{\partial \mathbf{E}}{\partial x}=\frac{\partial \mathbf{E}}{\partial \mathbf{Q}} \frac{\partial \mathbf{Q}}{\partial x} \quad, \quad \frac{\partial \mathbf{F}}{\partial y}=\frac{\partial \mathbf{F}}{\partial \mathbf{Q}} \frac{\partial \mathbf{Q}}{\partial y}
$$

Assim, ao substituir as derivadas dos vetores de fluxo, apresentadas acima pelas expressões (2.12), na equação (2.5), obtém-se a forma quase-linear das equações de Euler:

$$
\frac{\partial \mathbf{Q}}{\partial t}+\underbrace{\frac{\partial \mathbf{E}}{\partial \mathbf{Q}}}_{\mathbf{A}} \frac{\partial \mathbf{Q}}{\partial x}+\underbrace{\frac{\partial \mathbf{F}}{\partial \mathbf{Q}}}_{\mathbf{B}} \frac{\partial \mathbf{Q}}{\partial y}=0
$$

onde as matrizes Jacobianas A e $\mathbf{B}$ são dadas por:

$$
\mathbf{A}=\left[\begin{array}{cccc}
0 & 1 & 0 & 0 \\
\frac{(\gamma-1)\left(u^{2}+v^{2}\right)}{2}-u^{2} & (3-\gamma) u & (1-\gamma) v & (\gamma-1) \\
-u v & v & u & 0 \\
(\gamma-1) u\left(u^{2}+v^{2}\right)-\gamma u \frac{e}{\rho} & \gamma \frac{e}{\rho}-\frac{(\gamma-1)\left(3 u^{2}+v^{2}\right)}{2} & (1-\gamma) u v & \gamma u
\end{array}\right]
$$




$$
\mathbf{B}=\left[\begin{array}{cccc}
0 & 0 & 1 & 0 \\
-u v & v & u & 0 \\
\frac{(\gamma-1)\left(u^{2}+v^{2}\right)}{2}-v^{2} & (1-\gamma) u & (3-\gamma) v & (\gamma-1) \\
(\gamma-1) v\left(u^{2}+v^{2}\right)-\gamma u \frac{e}{\rho} & (1-\gamma) u v & \gamma \frac{e}{\rho}-\frac{(\gamma-1)\left(u^{2}+3 v^{2}\right)}{2} & \gamma v
\end{array}\right]
$$

Aqui, vale ressaltar que $\mathbf{E}$ e $\mathbf{F}$ são homogêneos ${ }^{1}$ de ordem 1 em relação a $\mathbf{Q}$. Desta forma, é possível afirmar que:

$$
\frac{\partial \mathbf{E}}{\partial \mathbf{Q}}=\mathbf{A} \Rightarrow \mathbf{E}=\mathbf{A Q} \quad \text { e } \quad \frac{\partial \mathbf{F}}{\partial \mathbf{Q}}=\mathbf{B} \Rightarrow \mathbf{F}=\mathrm{BQ}
$$

Observa-se que A e B são bastante complicadas. No entanto, as mesmas equações podem ser escritas em função de um vetor de variáveis primitivas $\mathbf{V}$ (que podem ser medidas diretamente), com matrizes Jacobianas mais simples, como mostra a equação abaixo (HIRSCH, 1988a):

$$
\frac{\partial \mathbf{V}}{\partial t}+\tilde{\mathbf{A}} \frac{\partial \mathbf{V}}{\partial x}+\tilde{\mathbf{B}} \frac{\partial \mathbf{V}}{\partial y}=0
$$

onde:

$$
\mathbf{V}=\left\{\begin{array}{c}
\rho \\
u \\
v \\
p
\end{array}\right\}, \tilde{\mathbf{A}}=\left[\begin{array}{cccc}
u & \rho & 0 & 0 \\
0 & u & 0 & 1 / \rho \\
0 & 0 & u & 0 \\
0 & \gamma p & 0 & u
\end{array}\right], \tilde{\mathbf{B}}=\left[\begin{array}{cccc}
v & 0 & 0 & 0 \\
0 & v & 0 & 0 \\
0 & 0 & v & 1 / \rho \\
0 & 0 & \gamma p & v
\end{array}\right]
$$

A relação entre as matrizes Jacobianas das variáveis conservadas e primitivas é dada pela seguinte transformação de similaridade:

$$
\begin{aligned}
\tilde{\mathbf{A}} & =\mathbf{M}^{-1} \mathbf{A M} \\
\tilde{\mathbf{B}} & =\mathbf{M}^{-1} \mathbf{B M}
\end{aligned}
$$

onde M, matriz Jacobiana da transformação das variáveis conservadas para as variáveis primitivas, e a sua inversa $\mathbf{M}^{-1}$ são definidas como:

$$
\mathbf{M}=\frac{\partial \mathbf{Q}}{\partial \mathbf{V}}=\left[\begin{array}{cccc}
1 & 0 & 0 & 0 \\
u & \rho & 0 & 0 \\
v & 0 & \rho & 0 \\
\frac{1}{2}\left(u^{2}+v^{2}\right) & \rho u & \rho v & \frac{1}{\gamma-1}
\end{array}\right]
$$

\footnotetext{
${ }^{1}$ Lomax, Pulliam e Zingg (2001) apresentam a propriedade homogênea das equaçōes de Euler de uma forma mais detalhada.
} 


$$
\mathbf{M}^{-1}=\frac{\partial \mathbf{V}}{\partial \mathbf{Q}}=\left[\begin{array}{cccc}
1 & 0 & 0 & 0 \\
-\frac{u}{\rho} & \frac{1}{\rho} & 0 & 0 \\
-\frac{v}{\rho} & 0 & \frac{1}{\rho} & 0 \\
\frac{1}{2}(\gamma-1)\left(u^{2}+v^{2}\right) & -(\gamma-1) u & -(\gamma-1) v & \gamma-1
\end{array}\right]
$$

Naturalmente, existem outras formas de representar as equações de Euler. Pode se escrevê-las através de qualquer combinação linear destas equações ou, em outras palavras, multiplicando-as por qualquer matriz inversível de mesma dimensão das matrizes Jacobianas. Observe que este procedimento altera as equações, mas não as variáveis dependentes, que ainda são conservadas (LANEY, 1998).

Uma vez que as equações de Euler são hiperbólicas, sempre é possível definir uma matriz $\mathbf{A}_{n}$ associada à um vetor $\vec{k}$ que possui autovalores reais e um conjunto completo de autovetores para qualquer direção deste vetor. Em outras palavras, o caráter hiperbólico das equações de Euler implica que a matriz $\mathbf{A}_{n}$ seja diagonalizável.

$$
\mathbf{A}_{n}=\vec{k} \cdot \overrightarrow{\mathbf{A}}=k_{x} \cdot \mathbf{A}+k_{y} \cdot \mathbf{B}
$$

onde $k_{x}$ e $k_{y}$ são, respectivamente, as componentes $x$ e $y$ do vetor $\vec{k}$ no sistema cartesiano bidimensional. Assim, a matriz $\mathbf{A}_{n}$ pode ser escrita da seguinte forma:

$$
\mathbf{A}_{n}=\left[\begin{array}{cc}
0 & k_{x} \\
k_{x}\left[\frac{(\gamma-1)\left(u^{2}+v^{2}\right)}{2}-u^{2}\right]-k_{y} u v & v k_{y}-(\gamma-3) u k_{x} \\
k_{y}\left[\frac{(\gamma-1)\left(u^{2}+v^{2}\right)}{2}-v^{2}\right]-k_{x} u v & v k_{x}-(\gamma-1) u k_{y} \\
\left(u k_{x}+v k_{y}\right)\left[(\gamma-1)\left(u^{2}+v^{2}\right)-\gamma \frac{e}{\rho}\right] & \gamma \frac{e}{\rho} k_{x}-(\gamma-1)\left[u v k_{y}+\frac{\left(3 u^{2}+v^{2}\right) k_{x}}{2}\right] \\
k_{y} & (\gamma-1) k_{x} \\
u k_{y}-(\gamma-1) v k_{x} & (\gamma-1) k_{y} \\
u k_{x}-(\gamma-3) v k_{y} & \gamma\left(u k_{x}+v k_{y}\right)
\end{array}\right]
$$

Observa-se que, apesar de $\mathbf{A}_{n}$ não ser simétrica, ela tem um conjunto completo de autovetores, isto é, possui 4 autovalores reais e 4 autovetores linearmente independentes.

A condição de hiperbolicidade das equações de Euler é expressa pela existência de soluções na forma de onda (HIRSCH, 1988b). Portanto, sua solução pode ser interpretada como uma série de ondas que se propagam no espaço carregando informação do escoamento. $\grave{A}$ cada autovalor $\sigma(\vec{k})$ da matriz $\mathbf{A}_{n}$ pode-se associar uma superfície característica, normal ao vetor $\vec{k}$, que indica a direção de propaga- 
ção de uma frente de onda, representando um aspecto de escoamentos invíscidos chamado propagação de perturbações. O estado físico de um dado ponto pode ser interpretado como resultado das quantidades transportadas ao longo das características. Isto implica que o sistema de equações pode ser reformulado como relações diferenciais escritas ao longo da frente de onda ou superfícies características apenas, conhecidas na literatura como formulação característica das equações de Euler. Os autovalores de $\mathbf{A}_{n}$ representam a velocidade de propagação da onda (HIRSCH, 1988b).

Utilizando a transformação apresentada anteriormente para tornar o sistema de equações função das variáveis primitivas, tem-se:

$$
\tilde{\mathbf{A}}_{n}=\mathbf{M}^{-1} \mathbf{A}_{n} \mathbf{M}
$$

É possível, então, diagonalizar este sistema de equações ao utilizar a seguinte relação:

$$
\begin{aligned}
\boldsymbol{\Lambda} & =\mathbf{L}^{-1} \tilde{\mathbf{A}}_{n} \mathbf{L} \\
& =\mathbf{L}^{-1} \mathbf{M}^{-1} \mathbf{A}_{n} \mathbf{M L}
\end{aligned}
$$

onde $\Lambda$ é uma matriz diagonal e $\mathbf{L}$ é tal que:

$$
\mathrm{L}^{-1}=\left[\begin{array}{cccc}
1 & 0 & 0 & -1 / c^{2} \\
0 & k_{y} & -k_{x} & 0 \\
0 & k_{x} & k_{y} & 1 / \rho c \\
0 & -k_{x} & -k_{y} & 1 / \rho c
\end{array}\right] \quad, \quad \mathbf{L}=\left[\begin{array}{cccc}
1 & 0 & \rho / 2 c & \rho / 2 c \\
0 & k_{y} & k_{x} / 2 & -k_{x} / 2 \\
0 & -k_{x} & k_{y} / 2 & -k_{y} / 2 \\
0 & 0 & \rho c / 2 & \rho c / 2
\end{array}\right]
$$

Assim, definindo as matrizes:

$$
\mathbf{P}=\mathbf{M L} \quad \text { e } \quad \mathbf{P}^{-1}=\mathbf{L}^{-1} \mathbf{M}^{-1}
$$

Que podem ser escritas de modo completo através das expressões:

$$
\begin{gathered}
\mathbf{P}=\left[\begin{array}{cccc}
1 & 0 & \rho / 2 c & \rho / 2 c \\
u & \rho k_{y} & \frac{\rho\left(c k_{x}+u\right)}{2 c} & \frac{\rho\left(u-c k_{x}\right)}{2 c} \\
v & -\rho k_{x} & \frac{\rho\left(c k_{y}+v\right)}{2 c} & \frac{\rho\left(v-c k_{y}\right)}{2 c} \\
\frac{\left(u^{2}+v^{2}\right)}{2} & \rho\left(u k_{y}-v k_{x}\right) & \frac{\rho}{2}\left(\frac{c}{\gamma-1}+\overrightarrow{\mathbf{v}} \cdot \vec{n}+\frac{u^{2}+v^{2}}{2 c}\right) & \frac{\rho}{2}\left(\frac{c}{\gamma-1}-\overrightarrow{\mathbf{v}} \cdot \vec{n}+\frac{u^{2}+v^{2}}{2 c}\right)
\end{array}\right] \\
\mathbf{P}^{-1}=\left[\begin{array}{cccc}
1-\frac{(\gamma-1)\left(u^{2}+v^{2}\right)}{2 c^{2}} & \frac{(\gamma-1) u}{c^{2}} & \frac{(\gamma-1) v}{c^{2}} & \frac{\gamma-1}{c^{2}} \\
\frac{v k_{x}-u k_{y}}{\rho} & \frac{k_{y}}{\rho} & \frac{-k_{x}}{\rho} & 0 \\
\frac{(\gamma-1)\left(u^{2}+v^{2}\right)-2 c\left(u k_{x}+v k_{y}\right)}{2 \rho c} & \frac{c k_{x}-(\gamma-1) u}{\rho c} & \frac{c k_{y}-(\gamma-1) v}{\rho c} & \frac{\gamma-1}{\rho c} \\
\frac{(\gamma-1)\left(u^{2}+v^{2}\right)+2 c\left(u k_{x}+v k_{y}\right)}{2 \rho c} & \frac{-c k_{x}-(\gamma-1) u}{\rho c} & \frac{-c k_{y}-(\gamma-1) v}{\rho c} & \frac{\gamma-1}{\rho c}
\end{array}\right]
\end{gathered}
$$


Então, a matriz $\mathbf{A}_{n}$ pode ser diagonalizada diretamente da seguinte maneira:

$$
\Lambda=\mathbf{P}^{-1} \mathbf{A}_{n} \mathbf{P}
$$

Resultando na matriz diagonal $\Lambda$, que pode ser escrita explicitamente como:

$$
\boldsymbol{\Lambda}=\left[\begin{array}{cccc}
k_{x} u+k_{y} v & 0 & 0 & 0 \\
0 & k_{x} u+k_{y} v & 0 & 0 \\
0 & 0 & k_{x} u+k_{y} v+c & 0 \\
0 & 0 & 0 & . k_{x} u+k_{y} v-c
\end{array}\right]
$$

Os dois primeiros autovalores são iguais à componente normal do vetor velocidade, $v_{n}$. Os dois autovalores restantes estão associados à ondas acústicas e são iguais à $v_{n} \pm c$. Então o sinal destes autovalores será determinado pela componente normal da velocidade na superfície de contorno.

Assim, de maneira a obter as equações de Euler decorrentes da diagonalização da matriz Jacobiana numa direção $\vec{k}$, pode-se pré-multiplicar a equação (2.15) por $\mathbf{L}^{-1}$ :

$$
\mathbf{L}^{-1} \frac{\partial \mathbf{V}}{\partial t}+\mathbf{L}^{-1} \tilde{\mathbf{A}} \frac{\partial \mathbf{V}}{\partial x}+\mathbf{L}^{-1} \tilde{\mathbf{B}} \frac{\partial \mathbf{V}}{\partial y}=0
$$

Mas, como $\mathbf{L L}^{-1}=\mathrm{I}$, pode--se introduzi-lo ao sistema de equações sem alterá-lo:

$$
\mathbf{L}^{-1} \frac{\partial \mathbf{V}}{\partial t}+\mathbf{L}^{-1} \tilde{\mathbf{A}} \mathbf{L} \mathbf{L}^{-1} \frac{\partial \mathbf{V}}{\partial x}+\mathbf{L}^{-1} \tilde{\mathbf{B}} \mathbf{L} \mathbf{L}^{-1} \frac{\partial \mathbf{V}}{\partial y}=0
$$

As relações (2.27) remetem à introdução de um novo conjunto de variáveis características W (HIRSCH, 1988b), também conhecidas como variáveis de Riemann, definidas pela relação de variações $\delta$ :

$$
\delta \mathbf{W}=\mathbf{L}^{-1} \delta \mathbf{V}=\left\{\begin{array}{c}
\delta w_{1} \\
\delta w_{2} \\
\delta w_{3} \\
\delta w_{4}
\end{array}\right\}=\left\{\begin{array}{c}
\delta \rho-\frac{1}{c^{2}} \delta p \\
k_{y} \delta u-k_{x} \delta v \\
\frac{1}{\rho c} \delta p-\left(k_{x} \delta u+k_{y} \delta v\right) \\
\frac{1}{\rho c} \delta p+\left(k_{x} \delta u+k_{y} \delta v\right)
\end{array}\right\}
$$

Apesar de $\mathbf{L}$ também ser uma matriz Jacobiana, esta não apresenta a propriedade homogênea, descrita no início desta seção, então:

$$
\mathbf{L} \equiv \frac{\partial \mathbf{V}}{\partial \mathbf{W}} \quad \operatorname{mas} \quad \mathbf{V} \neq \mathbf{L} \mathbf{W}
$$

Assim, as variáveis características só são definidas através das variações apresentadas pela equação (2.28). Ao utilizá-las para escrever o sistema de equações 
características, obtém-se:

$$
\frac{\partial \mathbf{W}}{\partial t}+\mathbf{L}^{-1} \tilde{\mathbf{A}} \mathbf{L} \frac{\partial \mathbf{W}}{\partial x}+\mathbf{L}^{-1} \tilde{\mathbf{B}} \mathbf{L} \frac{\partial \mathbf{W}}{\partial y}=0
$$

Que também pode ser apresentado com os termos expandidos, em função das variáveis primitivas, da seguinte forma:

$$
\left\{\begin{array}{rl}
\frac{\partial \rho}{\partial t}-\frac{1}{c^{2}} \frac{\partial p}{\partial t}= & -\left(\frac{\partial \rho}{\partial x}-\frac{1}{c^{2}} \frac{\partial p}{\partial x}\right) u-\left(\frac{\partial \rho}{\partial y}-\frac{1}{c^{2}} \frac{\partial p}{\partial y}\right) v \\
k_{y} \frac{\partial u}{\partial t}-k_{x} \frac{\partial v}{\partial t}= & \frac{1}{\rho}\left(k_{x} \frac{\partial p}{\partial y}-k_{y} \frac{\partial p}{\partial x}\right)-\left(k_{y} \frac{\partial u}{\partial x}-k_{x} \frac{\partial v}{\partial x}\right) u+ \\
& -\left(k_{y} \frac{\partial u}{\partial y}-k_{x} \frac{\partial v}{\partial y}\right) v
\end{array}=R_{1}\right.
$$

A formulação característica das equações de Euler é completamente equivalente à equação (2.5) apresentada anteriormente. A única diferença entre elas é o fato da formulação característica ter cada uma das equações relacionada a um autovalor (associado a uma direção de propagação $\vec{k}$ ). Assim, esta formulação é obtida de tal modo que as características sejam desacopladas. Em outras palavras, cada equação do sistema (2.31) corresponde, agora, à uma característica.

\subsection{Adimensionalização das Equações de Euler}

Ao adimensionalizar as variáveis das PDE's que governam o escoamento, valores de referência são agrupados na forma de parâmetros adimensionais, vistos como coeficientes nestas equações. Se os valores de referência forem apropriados, é possível escalar as variáveis adimensionais (dependentes e independentes) tipicamente com $\mathcal{O}(1)$. Desta forma, os termos das equações permitem a comparação de suas magnitudes e, conseqüentemente, identificar a importância de cada um deles em diferentes regimes, dando uma idéia dos mecanismos físicos que agem de forma dominante. Em particular, termos desprezíveis podem ser identificados, levando à simplificações em muitas circunstâncias.

A definição destes parâmetros adimensionais, que passam a caracterizar o escoamento, ainda torna possível utilizar a chamada análise de similaridade, que 
permite a extrapolação do desempenho de equipamentos de engenharia para outras condições de operação. Assim, é possível utilizar um modelo em escala reduzida para estudar o comportamento de um fluido ao redor de um objeto de grandes dimensões, desde que tenham os mesmos parâmetros adimensionais relevantes (FLETCHER, 1990b; FORTUNA, 2000). Eventualmente, a adimensionalização ainda pode levar à redução do número de parâmetros necessários para descrever o escoamento, facilitando assim a análise dos resultados.

Vale lembrar que equações adimensionalizadas de fenômenos distintos se tornam similares e permitem analogias como, por exemplo, a de Reynolds-Colburn que relaciona de troca de calor e quantidade de movimento. As respectivas grandezas dimensionais são obtidas ao se multiplicar os valores adimensionais pelos parâmetros dimensionais de referência. A título de ilustração, podem-se citar os números de Reynolds, Mach e Prandtl dentre os adimensionais mais relevantes na aerodinâmica. Contudo, no estudo de escoamentos invíscidos compressíveis, representados matematicamente pelas equações de Euler, apenas o número de Mach mantém sua relevância. Para os propósitos desta dissertação, propõe-se a definição dos seguintes adimensionais:

- Tempo adimensional

$$
t^{*}=t \frac{c_{\infty}}{\ell_{0}} \Rightarrow \frac{\partial()}{\partial t}=\frac{\partial t^{*}}{\partial t} \frac{\partial()}{\partial t^{*}}=\frac{c_{\infty}}{\ell_{0}} \frac{\partial()}{\partial t^{*}}
$$

onde $c_{\infty}$ é a velocidade do som do escoamento não perturbado e $\ell_{0}$ é um comprimento de referência.

- Comprimento adimensional

$$
\ell^{*}=\frac{\ell}{\ell_{0}} \Rightarrow \frac{\partial()}{\partial \ell}=\frac{\partial \ell^{*}}{\partial \ell} \frac{\partial()}{\partial \ell^{*}}=\frac{1}{\ell_{0}} \frac{\partial()}{\partial \ell^{*}}
$$

- Massa específica adimensional

$$
\rho^{*}=\frac{\rho}{\rho_{\infty}}
$$

onde $\rho_{\infty}$ é a massa específica do fluido do escoamento não perturbado.

- Velocidade adimensional

$$
u^{*}=\frac{u}{c_{\infty}} \quad, \quad v^{*}=\frac{v}{c_{\infty}}
$$

onde $u$ e $v$ correspondem, respectivamente, às componentes $x$ e $y$ do vetor velocidade em coordenadas cartesianas. 
- Pressão adimensional

$$
p^{*}=\frac{p}{\rho_{\infty} c_{\infty}^{2}}
$$

- Temperatura adimensional

$$
T^{*}=\frac{T}{T_{\infty}}
$$

- Energia adimensional

$$
e^{*}=\frac{e}{\rho_{\infty} c_{\infty}^{2}}
$$

É possível utilizá-los para reescrever o vetor de estados $\mathbf{Q}$, obtendo:

$$
\mathbf{Q}=\left\{\begin{array}{c}
\rho \\
\rho u \\
\rho v \\
e
\end{array}\right\}=\left\{\begin{array}{c}
\rho^{*} \rho_{\infty} \\
\rho^{*} \rho_{\infty} u^{*} c_{\infty} \\
\rho^{*} \rho_{\infty} v^{*} c_{\infty} \\
e^{*} \rho_{\infty} c_{\infty}^{2}
\end{array}\right\} \Rightarrow \frac{\partial \mathbf{Q}}{\partial t^{*}}=\frac{\rho_{\infty} c_{\infty}}{\ell_{0}} \frac{\partial}{\partial t^{*}}\left\{\begin{array}{c}
\rho^{*} \\
\left(\rho^{*} u^{*}\right) c_{\infty} \\
\left(\rho^{*} v^{*}\right) c_{\infty} \\
e^{*} c_{\infty}^{2}
\end{array}\right\}
$$

O mesmo pode ser feito ao vetor de fluxos da direção $x$, que resulta em:

$$
\begin{gathered}
\mathbf{E}=\left\{\begin{array}{c}
\rho u \\
\rho u^{2}+p \\
\rho u v \\
(e+p) u
\end{array}\right\}=\left\{\begin{array}{c}
\rho^{*} \rho_{\infty} u^{*} c_{\infty} \\
\rho^{*} \rho_{\infty} u^{* 2} c_{\infty}^{2}+p^{*} \rho_{\infty} c_{\infty}^{2} \\
\rho^{*} \rho_{\infty} u^{*} c_{\infty} v^{*} c_{\infty} \\
\left(e^{*} \rho_{\infty} c_{\infty}^{2}+p^{*} \rho_{\infty} c_{\infty}^{2}\right) u^{*} c_{\infty}
\end{array}\right\} \\
\Rightarrow \frac{\partial \mathbf{E}}{\partial x^{*}}=\frac{\rho_{\infty} c_{\infty}}{\ell_{0}} \frac{\partial}{\partial x^{*}}\left\{\begin{array}{c}
\rho^{*} u^{*} \\
\left(\rho^{*} u^{* 2}+p^{*}\right) c_{\infty} \\
\left(\rho^{*} u^{*} v^{*}\right) c_{\infty} \\
{\left[\left(e^{*}+p^{*}\right) u^{*}\right] c_{\infty}^{2}}
\end{array}\right\}
\end{gathered}
$$

Finalmente, o vetor de fluxos da direção y pode ser reescrito como:

$$
\begin{gathered}
\mathbf{F}=\left\{\begin{array}{c}
\rho v \\
\rho u v \\
\rho v^{2}+p \\
(e+p) v
\end{array}\right\}=\left\{\begin{array}{c}
\rho^{*} \rho_{\infty} v^{*} c_{\infty} \\
\rho^{*} \rho_{\infty} u^{*} c_{\infty} v^{*} c_{\infty} \\
\rho^{*} \rho_{\infty} v^{* 2} c_{\infty}^{2}+p^{*} \rho_{\infty} c_{\infty}^{2} \\
\left(e^{*} \rho_{\infty} c_{\infty}^{2}+p^{*} \rho_{\infty} c_{\infty}^{2}\right) v^{*} c_{\infty}
\end{array}\right\} \\
\Rightarrow \frac{\partial \mathbf{F}}{\partial y^{*}}=\frac{\rho_{\infty} c_{\infty}}{\ell_{0}} \frac{\partial}{\partial y^{*}}\left\{\begin{array}{c}
\rho^{*} v^{*} \\
\left(\rho^{*} u^{*} v^{*}\right) c_{\infty} \\
\left(\rho^{*} v^{* 2}+p^{*}\right) c_{\infty} \\
{\left[\left(e^{*}+p^{*}\right) v^{*}\right] c_{\infty}^{2}}
\end{array}\right\}
\end{gathered}
$$

Assim, as equações de Euler adimensionalizadas são dadas pela seguinte ex- 
pressão:

$$
\frac{\partial \mathbf{Q}^{*}}{\partial t^{*}}+\frac{\partial \mathbf{E}^{*}}{\partial x^{*}}+\frac{\partial \mathbf{F}^{*}}{\partial y^{*}}=0
$$

onde:

$$
\mathbf{Q}^{*}=\left\{\begin{array}{c}
\rho^{*} \\
\rho^{*} u^{*} \\
\rho^{*} v^{*} \\
e^{*}
\end{array}\right\} \quad, \quad \mathbf{E}^{*}=\left\{\begin{array}{c}
\rho^{*} u^{*} \\
\rho^{*} u^{* 2}+p^{*} \\
\rho^{*} u^{*} v^{*} \\
\left(e^{*}+p^{*}\right) u^{*}
\end{array}\right\} \quad, \quad \mathbf{F}=\left\{\begin{array}{c}
\rho^{*} v^{*} \\
\rho^{*} u^{*} v^{*} \\
\rho^{*} v^{* 2}+p^{*} \\
\left(e^{*}+p^{*}\right) v^{*}
\end{array}\right\}
$$

Com o objetivo de simplificar a notação, todos os asteriscos das variáveis adimensionais serão suprimidos, porém deve-se lembrar que daqui em diante trabalhar-se-á apenas com as variáveis adimensionalizadas.

É importante notar que a adimensionalização apresentada nesta seção é conveniente para escoamentos externos compressíveis. Veja que, no caso de simular escoamentos incompressíveis com número de Mach muito baixo, por exemplo, a equação de conservação de quantidade de movimento teria $\mathcal{O}(0)$, uma vez que a velocidade do som $\left(c_{\infty}\right)$ seria muito maior do que a velocidade do escoamento, enquanto a equação de conservação de massa permaneceria tendo $\mathcal{O}(1)$, resultando em erros numéricos ao resolver as equações de interesse. Desta forma, para simular outros tipos de escoamento, seria interessante utilizar uma adimensionalização diferente, escolhendo um estado de referência mais apropriado para cada situação. De qualquer forma, o procedimento de adimensionalização seria completamente análogo ao utilizado nesta seção (AZEVEDO, 2006).

\subsection{Método Numérico}

Ao longo dos anos, diversos métodos numéricos tem sido desenvolvidos para resolver equações diferenciais à derivadas parciais. Estes métodos podem ser agrupados, basicamente, em quatro grupos: métodos de elementos finitos, espectrais, de volumes finitos e de diferenças finitas. Na aerodinâmica, em particular, duas destas classes de métodos se destacam por serem muito exploradas. Os métodos que fazem aproximações numéricas das equações do movimento em sua forma integral como a equação (2.2) para encontrar sua solução são conhecidos na literatura por métodos de volumes finitos, enquanto os que fazem aproximações das equações em sua forma diferencial como a equação (2.5) são conhecidos por métodos de diferenças finitas (LOMAX; PULLIAM; ZINGG, 2001).

Os últimos aproximam as derivadas espaciais das equações que governam o 
escoamento por operadores obtidos à partir do truncamento de séries de Taylor. Assim, esses métodos são dependentes do sistema de coordenadas adotado e, portanto, para utilizá-los em malhas diferentes da cartesiana é necessário realizar uma transformação de coordenadas em que a malha do espaço transformado é cartesiana.

Por outro lado, os métodos de volumes finitos integram as equações em volumes de controle resultantes da discretização espacial. Assim, estes métodos não dependem do sistema de coordenadas adotado, mas da forma dos volumes de controle. Os balanços integrais das propriedades são obtidos a partir dos fluxos calculados através das faces dos volumes de controle. Estes métodos se tornaram populares em CFD basicamente por duas vantagens. Primeiro, eles asseguram que a discretização é conservativa, i.e., massa, quantidade de movimento e energia são conservadas em um senso discreto. Embora esta propriedade possa ser obtida utilizando uma formulação de diferenças finitas ao implementar as equações em forma conservativa, ela é obtida naturalmente à partir de uma formulação de volumes finitos. Segundo, métodos de volumes finitos não requerem uma transformação de coordenadas para que sejam aplicadas à malhas irregulares. Como resultado, elas podem ser aplicadas em malhas não-estruturadas consistindo de poliedros arbitrários em três dimensões ou em polígonos arbitrários em duas dimensões. Este aumento de flexibilidade pode ser usado com grande vantagem na geração de malhas em torno de geometrias arbitrárias (LOMAX; PULLIAM; ZINGG, 2001). Por estes motivos, esse método será adotado para a resolução das equações de Euler neste trabalho.

\subsubsection{Definição dos Volumes de Controle}

Como já foi visto, a representação matemática de escoamentos recai em sistemas de equações diferenciais à derivadas parciais. A resolução numérica destas equações pelo método dos volumes finitos requer a discretização espacial do domínio de interesse. Basicamente, existem duas classes de malhas utilizadas para realizar esta discretização: estruturadas e não-estruturadas. O que diferencia uma da outra é a maneira como a informação dos seus elementos é associada. Nas estruturadas, a conectividade de cada volume é diretamente conhecida na ordenação da malha. Tome como exemplo uma malha cartesiana bidimensional, em que o elemento $(i, j)$ tem como "vizinhos" os elementos $(i+1, j),(i-1, j)$, $(i, j+1),(i, j-1)$. Em malhas não-estruturadas, no entanto, não existe uma "regra" que relacione os índices dos volumes à sua posição no espaço. Desta forma, o 
elemento 4983 pode dividir arestas com os elementos 2, 784 e 5832, por exemplo. Conseqüentemente, a informação de conectividade deve ser armazenada à parte. Certamente, as malhas não-estruturadas apresentam maior custo computacional, mas tem grandes vantagens no tratamento de geometrias complexas. Assim, com a grande evolução dos recursos computacionais nas últimas décadas, a utilização deste tipo de malhas tem se tornado cada vez mais freqüente, já que se adaptam melhor à geometrias complexas, tornando o código mais geral.

Existem, no entanto, duas maneiras de lidar com as malhas computacionais, sejam elas estruturadas ou não. Pode-se utilizar um esquema centrado nas células (cell centered), no qual as propriedades são atribuídas ao centróide do elemento e avaliadas como a média da propriedade do mesmo, de maneira que os volumes sejam definidos explícitamente pela própria malha, ou ainda um esquema centrado nos vértices (cell vertex), onde as propriedades são atribuídas ao vértices dos volumes, que são gerados em torno dos vértices da malha. A figura 2.1 apresenta esquemas de ambos os tipos de discretização no caso de malhas não-estruturadas de triângulos. Observe que os volumes no esquema centrado nos vértices exigem a construção de uma malha (representada pelas linhas tracejadas) em cima de uma já existente (representada pelas linhas contínuas) e, se de um lado, a malha resultante possui menos elementos do que a original, o que resulta em menos equações a serem resolvidas, e não há necessidade de se utilizar volumes "fantasmas" para implementar condições de contorno, de outro, o número de faces a ser avaliado é muito maior. Mais do que isso, o número de faces por elemento pode variar, o que traz algumas dificuldades na implementação do código computacional.

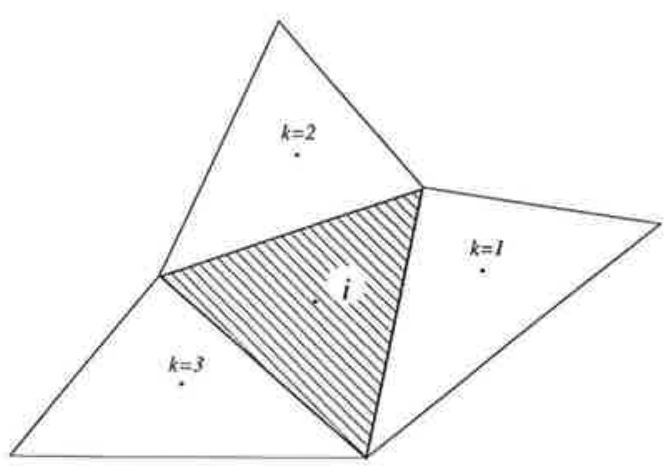

(a) Cell centered

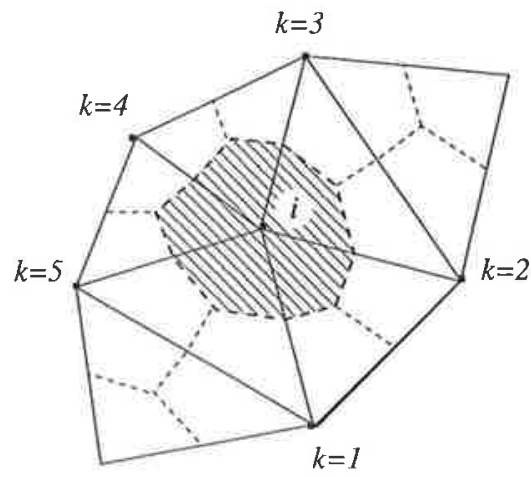

(b) Cell vertex

Figura 2.1: Comparação dos esquemas de discretização

Este trabalho não tem a pretensão de desenvolver um código computacional extremamente eficiente, portanto a utilização de um esquema de implementação mais simples, como o centrado nas células, é mais apropriada. Além disso Swanson e Radespiel (1991) mostraram que, utilizando as equações de Navier- 
Stokes como modelo físico do escoamento para escoamentos transônicos ao redor de aerofólios, ambos os esquemas apresentam soluções numéricas semelhantes.

\subsubsection{Discretização das Equações de Euler}

Considere as equações de Euler bidimensionais escritas em forma diferencial conservativa para coordenadas cartesianas apresentadas na equação (2.5). Definindo o vetor de fluxo "vetorial" $\overrightarrow{\mathcal{P}}$ como (AZEVEDo, 2006):

$$
\overrightarrow{\mathcal{P}} \equiv \mathbf{E} \hat{\imath}+\mathbf{F} \hat{\jmath}
$$

Pode-se substituir a expressão (2.44) nas equações de Euler para reescrevê--la utilizando o operador divergente $(\nabla)$ como:

$$
\frac{\partial \mathbf{Q}}{\partial t}+\nabla \cdot \overrightarrow{\mathcal{P}}=0
$$

Considere, agora, que se integre esta equação em um volume de controle euleriano (fixo no tempo) $\mathcal{V}$.

$$
\int_{\mathcal{V}} \frac{\partial \mathbf{Q}}{\partial t} \mathrm{~d} \mathcal{V}+\int_{\mathcal{V}}(\nabla \cdot \overrightarrow{\mathcal{P}}) \mathrm{d} \mathcal{V}=0
$$

Assim, as equações podem ser reescritas da seguinte maneira:

$$
\frac{\partial}{\partial t} \int_{\mathcal{V}} \mathbf{Q} \mathrm{d} \mathcal{V}+\int_{\mathcal{V}}(\nabla \cdot \overrightarrow{\mathcal{P}}) \mathrm{d} \mathcal{V}=0
$$

Como já foi dito anteriormente, esta forma de representação das equações de Euler não pode ser aplicada à uma descontinuidade. Portanto, utiliza-se o teorema de Gauss. Assim, é possível obter as equações que governam o escoamento do modo apresentado pelo sistema (2.2):

$$
\frac{\partial}{\partial t} \int_{\mathcal{V}} \mathbf{Q} \mathrm{d} \mathcal{V}+\int_{\mathcal{S}}(\overrightarrow{\mathcal{P}} \cdot \vec{n}) \mathrm{d} \mathcal{S}=0
$$

onde $\vec{n}$ é a normal unitária apontada para fora do volume de controle.

Observe que, neste caso, utilizando o teorema do gradiente, esta última equação poderia ser escrita simplesmente como:

$$
\frac{\partial}{\partial t} \int_{\mathcal{V}} \mathbf{Q} \mathrm{d} \mathcal{V}+\int_{\mathcal{S}}(\mathbf{E} \mathrm{d} y-\mathbf{F} \mathrm{d} x)=0
$$

Considerando que as equações serão discretizadas em um contexto de volumes finitos centrado nas células, definem-se as propriedades de um volume de 
controle como sendo a média das propriedades naquele volume. Assim, o vetor de variáveis conservadas discreto do $i$-ésimo elemento pode ser escrito como uma média volumétrica (AZEVEDO, 2006):

$$
\mathrm{Q}_{i}=\frac{1}{\mathcal{V}_{i}} \int_{\mathcal{V}_{i}} \mathrm{Q} \mathrm{d} x \mathrm{~d} y
$$

Desta forma, as equações podem ser reescritas para o volume $\mathcal{V}_{i}$ da célula $i$ como:

$$
\frac{\partial}{\partial t}\left(\mathcal{V}_{i} \mathbf{Q}_{i}\right)+\int_{\mathcal{S}_{i}}(\mathbf{E} \mathrm{d} y-\mathbf{F} \mathrm{d} x)=0
$$

O processo de discretização espacial da integral de superfície na equação acima recai em um operador convectivo usualmente referenciado como $C\left(\mathbf{Q}_{i}\right)$. Para uma malha não-estruturada de triângulos, este operador pode ser escrito da seguinte maneira (AZEVEDO, 2006):

$$
C\left(\mathbf{Q}_{i}\right)=\sum_{k=1}^{3}\left[\mathbf{E}\left(\mathbf{Q}_{i k}\right) \Delta y_{i k}-\mathbf{F}\left(\mathbf{Q}_{i k}\right) \Delta x_{i k}\right] \cong \int_{\mathcal{S}_{i}}(\mathbf{E} \mathrm{d} y-\mathbf{F ~ d} x)
$$

onde,

$$
\mathrm{Q}_{i k}=\frac{1}{2}\left(\mathbf{Q}_{i}+\mathbf{Q}_{k}\right)
$$

É importante observar que o método de Jameson, implementado neste trabaIho, avalia propriedades na face formada entre os elementos $i$ e $k$ através de um esquema centrado como mostra a equação (2.53).

\subsubsection{Avaliação de Derivadas Espaciais}

A avaliação de derivadas espaciais em malhas cartesianas por diferenças finitas é intuitiva. Entretanto, a utilização um método de volumes finitos em um domínio discretizado por malhas não-estruturadas exige cálculos um pouco mais elaborados. Para determiná-las é interessante utilizar novamente o conceito de como são definidas as propriedades discretas em um volume de controle (AZEVEDO, 2006). De maneira análoga, podem-se definir derivadas médias nas direçôes cartesianas $x$ e $y$ de uma propriedade qualquer $\beta$, por exemplo, no interior de um volume $\mathcal{V}_{i}$ como sendo as integrais:

$$
\begin{aligned}
& \left(\frac{\partial \beta}{\partial x}\right)_{i}=\frac{1}{\mathcal{V}_{i}} \int_{\mathcal{V}_{i}}\left(\frac{\partial \beta}{\partial x}\right) \mathrm{d} \mathcal{V} \\
& \left(\frac{\partial \beta}{\partial y}\right)_{i}=\frac{1}{\mathcal{V}_{i}} \int_{\mathcal{V}_{i}}\left(\frac{\partial \beta}{\partial y}\right) \mathrm{d} \mathcal{V}
\end{aligned}
$$


Utilizando o teorema de Gauss, é possível eliminar as derivadas contidas nas integrais do volume, transformando-a em integrais de superfície. Assim:

$$
\begin{aligned}
& \left(\frac{\partial \beta}{\partial x}\right)_{i}=\frac{1}{\mathcal{V}_{i}} \int_{\mathcal{S}_{i}} \beta \mathrm{d} y \\
& \left(\frac{\partial \beta}{\partial y}\right)_{i}=-\frac{1}{\mathcal{V}_{i}} \int_{\mathcal{S}_{i}} \beta \mathrm{d} x
\end{aligned}
$$

Seguindo a mesma idéia da discretização das equações de Euler, estas derivadas podem ser aproximadas numericamente através das seguintes expressões:

$$
\begin{aligned}
& \left(\frac{\partial \beta}{\partial x}\right)_{i} \cong \frac{1}{\mathcal{V}_{i}} \sum_{k} \frac{1}{2}\left(\beta_{i}+\beta_{k}\right) \Delta y_{i k} \\
& \left(\frac{\partial \beta}{\partial y}\right)_{i} \cong-\frac{1}{\mathcal{V}_{i}} \sum_{k} \frac{1}{2}\left(\beta_{i}+\beta_{k}\right) \Delta x_{i k}
\end{aligned}
$$

\subsubsection{Dissipação Artificial}

Segundo Hirsch (1988b), equações hiperbólicas admitem solução na forma de onda (wave-like solutions). Assim, pode-se fazer uma análise de Fourier em termos de variáveis primitivas, como velocidade ou pressão, para determinar o comportamento da solução das equações de Euler.

Para resolver numericamente o sistema de equações diferenciais, num certo domínio, é necessário discretizá-lo espacialmente. Por este motivo, não é possível resolver numericamente todos os modos que representam uma descontinuidade que, por exemplo, necessita um número infinito de modos para ser descrita do ponto de vista de uma análise de Fourier, como mostra a figura 2.2. Isto ocorre porque a capacidade de capturar o espectro de freqüências é limitada pela escala de comprimento do tamanho dos volumes da malha. Assim, nestes casos existem modos acima de um número de corte $N_{\text {corte }}$ associada a malha que não são captados pela solução numérica.

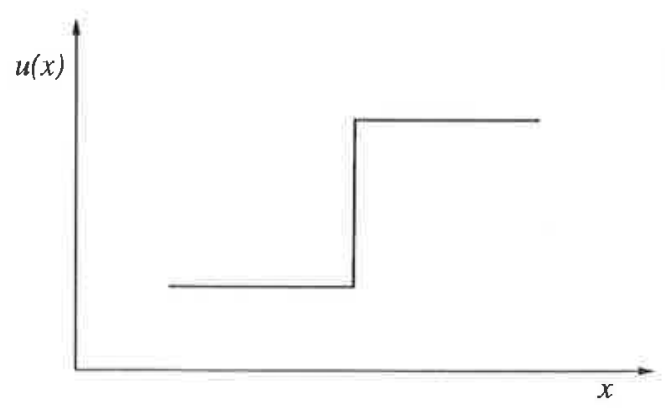

(a) Choque

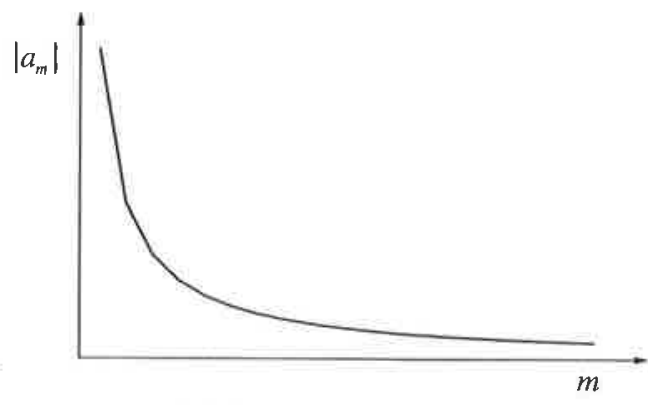

(b) Espectro de onda

Figura 2.2: Análise de Fourier de um choque unidimensional 
Para entender melhor a necessidade da dissipação artificial em escoamentos invíscidos, Fletcher (1990b) propõe a análise de uma descontinuidade presente na solução das equações de Euler unidimensionais apresentada pela figura 2.2(a). A série de Fourier em $u(x)$ que representa esta solução é dada por:

$$
u(x)=\sum_{m} a_{m} e^{(i m x)}
$$

onde $m$ é o número de onda e $a_{m}$ é a amplitude da $m$-ésima componente.

Devido aos termos convectivos não-lineares, quando a solução $u$ é interpretada como uma série de Fourier, o termo convectivo $u \frac{\partial u}{\partial x}$ é visto introduzindo o produto (FLETCHER, 1990b):

$$
u \frac{\partial u}{\partial x} \rightarrow i \sum_{m} a_{m} e^{i m x} \sum_{l} l a_{l} e^{i l x}
$$

que implica no aparecimento de números de onda $m-l$ e $m+l$. Este fenômeno, conhecido como cascateamento de freqüência, faz com que a solução discreta da solução transiente gere ondas de alta $(m+l)$ e baixa freqüência $(m-l)$. As baixas freqüências não causam qualquer problema. Entretanto, a constante geração de números de onda maiores a cada passo de tempo pode levá-los a ultrapassar o número de onda de corte. Estes números de onda são então adicionados, espuriamente, aos números de onda "resolvíveis". Em cálculos numéricos não se pode ignorá-los e deve-se levá-los em conta no algorítimo construído, já que a reconstrução da solução indica que um erro de aliasing está sendo introduzido, degradando assim a precisão e estabilidade dos cálculos (FLETCHER, 1990b).

Em problemas reais, a produção de altas freqüências é eventualmente limitada pela viscosidade. Entretanto, quando se resolve as equações de Euler, não existem mecanismos físicos para dissipá-las. Então todo método numérico que visa resolver estas equações deve conter alguma forma de dissipação inerente para limitar a produção de modos de alta freqüência. Isto pode ser feito em uma infinidade de modos (PULliAM, 1985).

Uma vez que a adição de dissipação numérica é equivalente a introduzir intencionalmente um comportamento não-físico é necessário controlá-la cuidadosamente para que o erro introduzido não seja excessivo (LOMAX; PULLIAM; ZINGG, 2001). Para resolver este problema é necessário dissipar apenas os modos "não resolvíveis" maiores do que $N_{\text {corte }}$ (ou uma região próxima à este número). Isto pode ser feito através da inclusão de dissipação pelo método numérico, conhecida na literatura como dissipação artificial ou dissipação numérica. Ela é indispensável para se garantir a estabilidade numérica de problemas não-lineares dominados 
por convecção (AZEVEDO, 2006). A natureza, quantidade e forma desta dissipação determinarão a precisão e realizabilidade da solução. O efeito da adição de dissipação artificial no espectro de onda da solução pode ser visto esquematicamente na figura 2.3 .

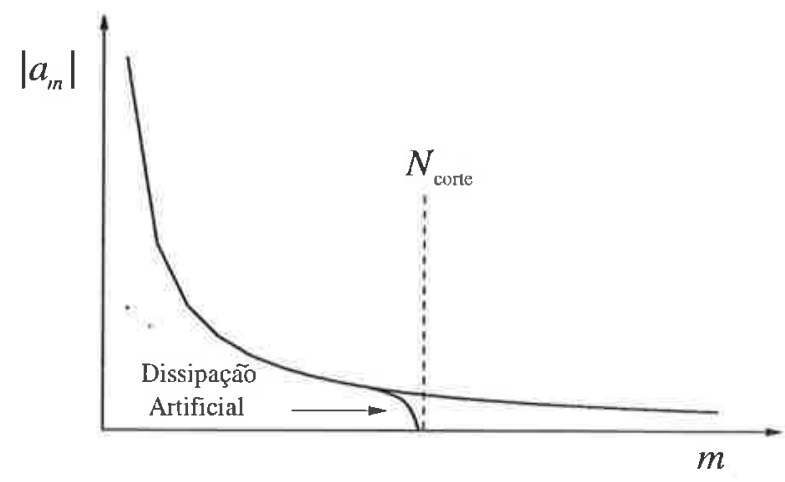

Figura 2.3: Efeito da adição de dissipação artificial no espectro de onda

É claro que mesmo em escoamentos de altos números de Reynolds, longe de choques, também é necessária a inclusão de termos de dissipação artificial. Porém, neste caso o nível de dissipação artificial necessário para controlar aliasing é muito menor do que em choques, já que as amplitudes do espectro de solução são muito menores. Portanto, em regiões essencialmente invíscidas, a dissipação numérica deve ser suficientemente pequena de modo que o balanço entre os outros termos das equações governantes não seja alterado, i.e., que a solução não seja afetada.

Ao discretizar as equações de Euler, obtém-se uma equação modificada que não corresponde mais ao problema original. Ao se escrever esta equação modificada através de uma série de Taylor, verifica-se que esta corresponde ao problema original acrescido de infinitos termos de ordem menor. Desta forma insere-se à solução um erro de aproximação numérica. Em métodos upwind alguns destes termos provocam alteração na amplitude da solução, dissipando-a. A discretização das equações de Euler por métodos de diferenças centradas, no entanto, produz uma PDE modificada que não possui termos que alteram a amplitude, mas apenas a fase da solução (LOMAX; PULLIAM; ZINGG, 2001; AZEVEDO, 2006). Assim, em problemas não-lineares onde se utilizam diferenças centradas, os termos de dissipação artificial devem ser introduzidos explícitamente para controlar as instabilidades não-lineares descritas anteriormente. Por outro lado, a sua existência intrínseca em métodos upwind "justifica" observações encontradas algumas vezes na literatura de que "métodos upwind não precisam de dissipação artificial" (AZEVEDO, 2006).

Operador dissipação artificial utilizado nesta dissertação, baseado no trabalho 
de Azevedo e Dourado (1991), é dado pela seguinte expressão:

$$
D\left(\mathbf{Q}_{i}\right)=d^{(2)}\left(\mathbf{Q}_{i}\right)+d^{(4)}\left(\mathbf{Q}_{i}\right)
$$

O operador Laplaciano não-dividido, $d^{(2)}\left(\mathbf{Q}_{i}\right)$, que fornece estabilidade numérica na presença de ondas de choque é dado por:

$$
d^{(2)}\left(\mathbf{Q}_{i}\right)=\sum_{k}\left[\frac{1}{2}\left(A_{i}+A_{k}\right) \epsilon_{i k}^{(2)}\left(\mathbf{Q}_{k}-\mathbf{Q}_{i}\right)\right]
$$

onde,

$$
\epsilon_{i k}^{(2)}=k^{(2)} \max \left(\nu_{i}, \nu_{k}\right) \quad \text { e } \quad \nu_{i}=\frac{\sum_{k}\left|p_{k}-p_{i}\right|}{\sum_{k}\left(p_{k}+p_{i}\right)}
$$

Enquanto o operador bi-harmônico, $d^{(4)}\left(\mathbf{Q}_{i}\right)$, que fornece estabilidade de campo nas regiões "suaves" do escoamento é escrito como:

$$
d^{(4)}\left(\mathbf{Q}_{i}\right)=\sum_{k}\left[\frac{1}{2}\left(A_{i}+A_{k}\right) \epsilon_{i k}^{(4)}\left(\nabla^{2} \mathbf{Q}_{k}-\nabla^{2} \mathbf{Q}_{i}\right)\right]
$$

onde,

$$
\epsilon_{i k}^{(4)}=\max \left[0,\left(k^{(4)}-\epsilon_{i k}^{(2)}\right)\right] \quad \text { e } \quad \nabla^{2} \mathbf{Q}_{i} \equiv \sum_{k}\left(\mathbf{Q}_{k}-\mathbf{Q}_{i}\right)
$$

O termo $\frac{1}{2}\left(A_{i}+A_{k}\right)$ é um fator de escalonamento dos termos de dissipação artificial. Neste modelo de dissipação artificial proposto, faz-se este termo proporcional à magnitude do maior autovalor (velocidade característica) das equações de Euler na direção normal à face.

$$
A_{i}=\sum_{k}\left[\left|\overrightarrow{\mathbf{v}}_{i k} \cdot \vec{S}_{i k}\right|+a_{i k}\left|\vec{S}_{i k}\right|\right]
$$

onde $\overrightarrow{\mathbf{v}}_{i k}$ é a velocidade do fluido na face que une os elementos $i$ e $k$ dada por:

$$
\overrightarrow{\mathbf{v}}_{i k}=u_{i k} \hat{i}_{x}+v_{i k} \hat{i}_{y}
$$

As constantes utilizadas são as mesmas propostas por Jameson, Schimidt e Turkel (1981), $k^{(2)}=1 / 4$ e $k^{(4)}=3 / 256$.

Note que o termo $\nu_{i}$ atua como uma "chave" para a dissipação artificial, já que fornece uma medida do gradiente de pressão. Ele é, portanto, responsável por manter o operador Laplaciano não-dividido "ligado" em regiões onde há descontinuidades, enquanto o operador bi-harmônico permanece "desligado". Por outro 
lado, em regiões "suaves" do escoamento, onde o gradiente de pressão é baixo, ele "liga" o operador bi-harmônico para garantir estabilidade e "desliga" o operador Laplaciano não-dividido para não degradar a solução.

Observe ainda que a inclusão de termos de dissipação numérica cria a possibilidade de geração de entropia nas equações de Euler. Isto permite identificar uma das propriedades mais importantes das soluções numéricas em CFD, os chamados efeitos "visíveis" da dissipação numérica (HIRSCH, 2007). Assim, ao utilizar as equações de Euler como modelo da física do escoamento, é possível identificar regiões onde a dissipação tem influência na solução numérica. Este fato pode ser um guia na direção de refinamento de malha, de modo a trazer este efeito em um nível aceitável.

As equações completamente discretizadas no espaço, já com os termos de dissipação artificial incluídos, assumindo uma malha estacionária, podem ser, então, escritas como:

$$
\frac{\mathrm{d} \mathbf{Q}_{i}}{\mathrm{~d} t}=-\frac{1}{\mathcal{V}_{i}}\left[C\left(\mathbf{Q}_{i}\right)-D\left(\mathbf{Q}_{i}\right)\right]
$$

Observe que isto representa, portanto, um conjunto de equações diferenciais ordinárias ( $\mathrm{ODE}^{2}$ 's) no tempo que pode ser integrado por qualquer método de marcha no tempo.

\subsubsection{Esquema de Marcha no Tempo}

A resolução numérica das equações que governam o escoamento em domínios discretizados por malhas estruturadas recai em matrizes esparsas, mas de banda estreita, o que facilita a sua "inversão" para resolver o sistema de equações. Entretanto, em malhas não-estruturadas, estas matrizes continuam esparsas, mas não de banda estreita. Portanto, sua "inversão" fica mais custosa, o que dificulta a implementação de métodos implícitos. Assim, mesmo na literatura, encontra-se uma preferência por métodos explícitos de marcha no tempo. Por este motivo o método Runge-Kutta de 5 passos e $2^{\mathrm{a}}$ ordem de precisão sugerido por Jameson e Mavriplis (1986) foi escolhido para discretizar a derivada temporal do sistema (2.63). Note que o com a utilização de um método de marcha no tempo RungeKuta de 5 passos seria possível obter até $5^{\mathrm{a}}$ ordem de precisão. Entretanto, a escolha dos coeficientes $\alpha_{i}$ apresentada troca a precisão por um aumento da região de estabilidade (AZEVEDO, 2006).

Por questões de economia de processamento o operador dissipação só é cal-

\footnotetext{
${ }^{2}$ sigla em inglês para Ordinary Differential Equation
} 
culado até o segundo estágio. Esta discretização resulta no seguinte método numérico de marcha no tempo:

$$
\begin{aligned}
& \mathrm{Q}_{i}^{(0)}=\mathrm{Q}_{i}^{(n)} \\
& \mathbf{Q}_{i}^{(1)}=\mathbf{Q}_{i}^{(0)}-\alpha_{1} \frac{\Delta t_{i}}{\mathcal{V}_{i}}\left[C\left(\mathbf{Q}_{i}^{(0)}\right)-D\left(\mathbf{Q}_{i}^{(0)}\right)\right] \\
& \mathbf{Q}_{i}^{(2)}=\mathbf{Q}_{i}^{(0)}-\alpha_{2} \frac{\Delta t_{i}}{\mathcal{V}_{i}}\left[C\left(\mathbf{Q}_{i}^{(1)}\right)-D\left(\mathbf{Q}_{i}^{(1)}\right)\right] \\
& \mathrm{Q}_{i}^{(3)}=\mathrm{Q}_{i}^{(0)}-\alpha_{3} \frac{\Delta t_{i}}{\mathcal{V}_{i}}\left[C\left(\mathrm{Q}_{i}^{(2)}\right)-D\left(\mathbf{Q}_{i}^{(1)}\right)\right] \\
& \mathrm{Q}_{i}^{(4)}=\mathrm{Q}_{i}^{(0)}-\alpha_{4} \frac{\Delta t_{i}}{\mathcal{V}_{i}}\left[C\left(\mathrm{Q}_{i}^{(3)}\right)-D\left(\mathrm{Q}_{i}^{(1)}\right)\right] \\
& \mathrm{Q}_{i}^{(5)}=\mathrm{Q}_{i}^{(0)}-\alpha_{5} \frac{\Delta t_{i}}{\mathcal{V}_{i}}\left[C\left(\mathbf{Q}_{i}^{(4)}\right)-D\left(\mathbf{Q}_{i}^{(1)}\right)\right] \\
& \mathrm{Q}_{i}^{(n+1)}=\mathrm{Q}_{i}^{(5)}
\end{aligned}
$$

onde os coeficientes utilizados são $\alpha_{1}=\frac{1}{4}, \alpha_{2}=\frac{1}{6}, \alpha_{3}=\frac{3}{8}, \alpha_{4}=\frac{1}{2}, \alpha_{5}=1$.

Como, neste caso, só se tem interesse na solução em estado estacionário, pode-se utilizar volumes com passo de tempo variável com a condição de $\mathrm{CFL}^{3}$ (COURANT; FRIEDRICHS; LEWY, 1967), definida na equação (2.64) e que nada mais é do que a velocidade com que a informação se propaga no tempo adimensionalizada. Observe, no entanto, que ao utilizar esta condição, as soluções intermediárias (transiente) não fazem mais sentido fisicamente.

$$
C F L=\frac{c \cdot \Delta t}{\Delta l}
$$

Esta condição de estabilidade fundamental na maioria dos esquemas explícitos de marcha no tempo para equações de onda e convecção expressa que a distância "coberta" durante o intervalo de tempo $\Delta t$, por perturbações com velocidade $c$, deveriam ser menores do que a menor distância entre dois pontos da malha. Em outras palavras, a condição de estabilidade expressa que a razão de malha $\Delta t / \Delta x$ tem que ser escolhida de tal forma que o domínio de dependência da equação diferencial esteja contido no domínio numérico de dependência das equações discretizadas, ou seja, o esquema numérico definindo a aproximação $u_{i}^{n+1}$ no ponto $i$ da malha deve ser capaz de incluir toda informação física que influencia o comportamento do sistema neste ponto. Se isto não ocorrer, então a variação nas condições físicas em regiões dentro do domínio de dependência físico, mas fora do domínio de dependência numérico não seriam vistas pelo esquema numérico e, portanto, a diferença entre a solução exata e a solução numérica poderia se tornar grande (HIRSCH, 2007).

\footnotetext{
${ }^{3}$ número de Courant-Friedrichs-Tewy
} 
Fortuna (2000) mostra que ao tornar o domínio de dependência numérico maior do que o domínio de dependência físico, a diminuição de CFL implica no aumento da dissipação numérica, aumentando assim a estabilidade do método.

Portanto, o passo de tempo utilizado em um volume $i$ é dado por:

$$
\Delta t_{i}=\frac{\Delta l_{i} \cdot C F L}{v_{\text {char }}}
$$

onde $v_{\text {char }}=(|\overrightarrow{\mathbf{v}}|+c)_{i}$ corresponde ao maior autovalor das matrizes jacobianas, ou seja a maior velocidade de propagação de perturbações, e $\Delta l_{i}$ corresponde à dimensão da menor aresta do volume $i$.

\subsection{Condições de Contorno}

Uma equação diferencial (seja ela ordinária ou à derivadas parciais) admite infinitas soluções. No entanto, na maioria das aplicações, não se tem interesse em encontrar a solução geral desta equação (ou sistema de equações), mas uma solução particular definida pelas condições iniciais e de contorno. Assim, uma solução particular deve satisfazer a PDE em todo o domínio e as suas respectivas condições de contorno em todos os pontos da fronteira (HADAMARD, 1952).

Portanto, a grande questão na resolução de PDE's reside no problema de contorno, que consiste em determinar uma solução do sistema de equações de modo a satisfazer uma PDE "indefinida" sob condiçôes de contorno "definidas". De acordo com Hadamard (1952), o problema estará "corretamente ajustado" se as condições auxiliares (iniciais e de contorno) forem tais que determinem uma e somente uma solução da PDE, o que vai ao encontro da própria definição de problema matematicamente bem-posto que, segundo o próprio Jacques Hadamard, deve satisfazer as seguintes condições (FLETCHER, 1990a):

1. a solução existe;

2. a solução é única;

3. a solução depende continuamente das condições iniciais e de contorno.

No caso das equações de Euler, a grande dificuldade para se garantir que o problema é bem-posto reside na questão da unicidade da sua solução. Segundo Fletcher (1990a), a causa usual de não-unicidade se deve às condições auxiliares não estarem condizentes com a PDE que se quer resolver. As condições de contorno apropriadas numa parede sólida são bem conhecidas, mas existe alguma 
flexibilidade ao fazer a escolha certa das condições de contorno de farfield. Em geral, uma subespecificação das condições de contorno leva a não-unicidade e uma sobrespecificação à soluções não-físicas (FLETCHER, 1990a).

A questão de problema bem-posto, no entanto, ainda é "aberta" e não se tem garantias de que as equações de Euler bidimensionais sejam bem-postas para quaisquer condições iniciais e de contorno. Jameson (1991) apresenta, inclusive, alguns aerofólios que apresentaram múltiplas soluções numéricas em seu código. Entretanto, estes casos foram verificados apenas à uma faixa estreita de números de Mach e ângulos de ataque. É importante salientar que em todos estes casos citados por Jameson havia a presença de ondas de choque e que o modelo Euler, apesar de capturar choques, não garante que a segunda lei da termodinâmica seja satisfeita.

Assim, não há nenhuma garantia que as equações de Euler sejam sempre bempostas. No entanto, ao utilizar uma formulação características para implementar as condições de contorno deste problema consegue se, ao menos, evitar que o problema esteja sub ou sobrespecificado, tornando o problema tão bem-posto quanto a literatura indica (HIRSCH, 1988a; HIRSCH, 1988b).

Observe que sempre é possível decompor a propagação de uma característica em uma componente paralela à fronteira do domínio e outra normal à ela. A primeira descreve a informação que permanece na superfíce de fronteira, enquanto a última, por sua vez, representa a informação que efetivamente "entra" ou "sai" do domínio (HIRSCH, 1988b).

Características que "entram" no domínio carregam informação da fronteira para o interior do domínio. Por outro lado, em características que "saem" do domínio a informação interna alcança a fronteira. Entretanto, apenas variáveis transportadas da fronteira em direção ao interior podem ser livremente impostas nas fronteiras como condições de contorno físicas (HIRSCH, 1988a). As variáveis restantes dependerão das propriedades do escoamento computado no domínio e são, portanto, parte da solução. Contudo, do ponto de vista numérico, de modo a calcular as propriedades numa célula de contorno é necessário saber todas as propriedades do volume "fantasma" adjacente a ela, além das condições físicas permitidas. Esta informação adicional, chamada por Hirsch (1988a) de condiçâa de contorno numérica ${ }^{4}$, deve ser consistente com as propriedades físicas do escoamento, bem como compatível com as equações discretizadas e tem que ser

\footnotetext{
${ }^{4} \hat{E}$ importante salientar que, apesar do nome utilizado na literatura, esta condição de contorno não tem origem numérica, mas física, já que é obtida à partir das equações que governam o escoamento.
} 
adicionados à fronteira física de modo a definir completamente o problema numérico.

Um importante efeito do procedimento das condições de contorno numéricas aplicado às fronteiras permeáveis é assegurar que perturbaçôes geradas no domínio computacional, por exemplo, o transiente em um escoamento em estado estacionário, deixem o domínio sem que sejam refletidas nas fronteiras, uma vez que estas fronteiras não são entidades físicas, apenas abstrações matemáticas. Isto implica que a propagação destas perturbações seja compatível com as propriedades de propagação características das equações de Euler (HIRSCH, 1988b).

Quando isto não ocorre, a precisão dos cálculos pode ser fortemente afetada pela reflexão ocorrida na fronteira. É portanto recomendado aplicar, as equações características como procedimentos de contorno (HIRSCH, 1988b). Um pouco de reflexão pode até existir e ter efeitos em taxa de convergência, reduzindo-se ao longo da convergência em si.

Uma vez que as condições de contorno de uma fronteira estão associadas às características normais à ela, pode-se tomar, por mera conveniência, o vetor $\vec{k}$ do sistema (2.31) como sendo normal à superfície de fronteira e apontando para dentro do domínio, por exemplo, como indica a figura 2.4. Desta forma, podemse utilizar as equações (2.31) para determinar as quantidades transportadas do domínio do escoamento para a fronteira e assim "completar" a informação proveniente de características vindas do escoamento não-perturbado nos volumes "fantasmas".

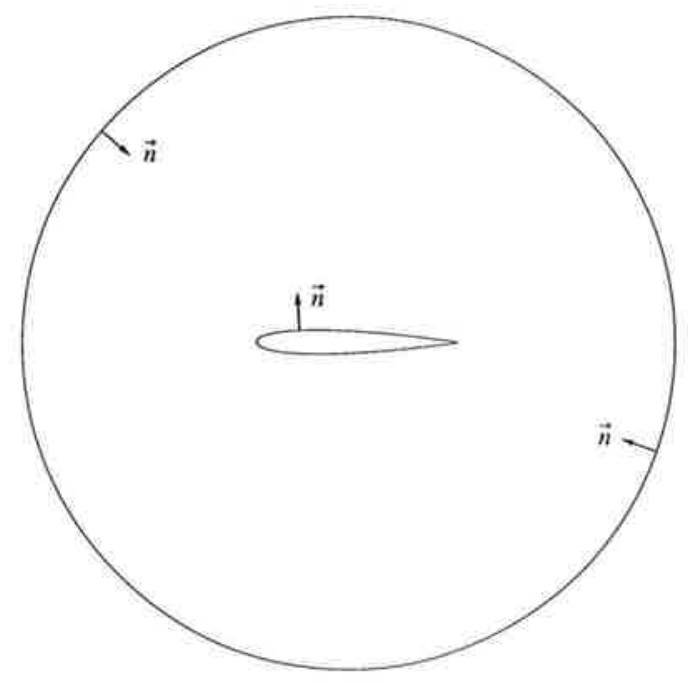

Figura 2.4: Vetores normais nas fronteiras do domínio

Em princípio, as condições físicas a serem impostas seriam a entropia ou as variáveis de Riemann. Entretanto, isto não é necessariamente muito prático, 
uma vez que estas variáveis não são geralmente conhecidas. Ao invés disso, condições que são fixadas em situações práticas em experimentos são velocidades e pressões e, portanto, a informação característica pode ter de ser definida de uma maneira iterativa ou aproximada, particularmente em fronteiras subsônicas (HIRSCH, 1988b).

Pode-se verificar a partir de um ponto de vista teórico, bem como por meio de experimentos numéricos que a escolha das condições de contorno numéricas pode ter um efeito dominante na precisão, estabilidade e taxa de convergência de muitos esquemas (HIRSCH, 1988b).

\subsubsection{Os Volumes "Fantasmas"}

A escolha de uma formulação de volumes finitos com esquema centrado nas células no método numérico para a resolução das equações que governam o escoamento naturalmente introduz uma dificuldade na implementação das condições de contorno. Observe que os volumes de fronteira não possuem "vizinhos" adjacentes à todas as suas arestas. Este fato remete à utilização de volumes "fantasmas" (ghost cells) para a implementação das condições de contorno. Estes são volumes fictícios que não pertencem ao domínio de interesse, mas são contíguos aos volumes de fronteira e construídos à imagem e semelhança destes. Além disso, os valores das propriedades nos volumes "fantasmas" não são necessariamente físicos (realizáveis), apenas servem o propósito de impor as condições de contorno da fronteira.

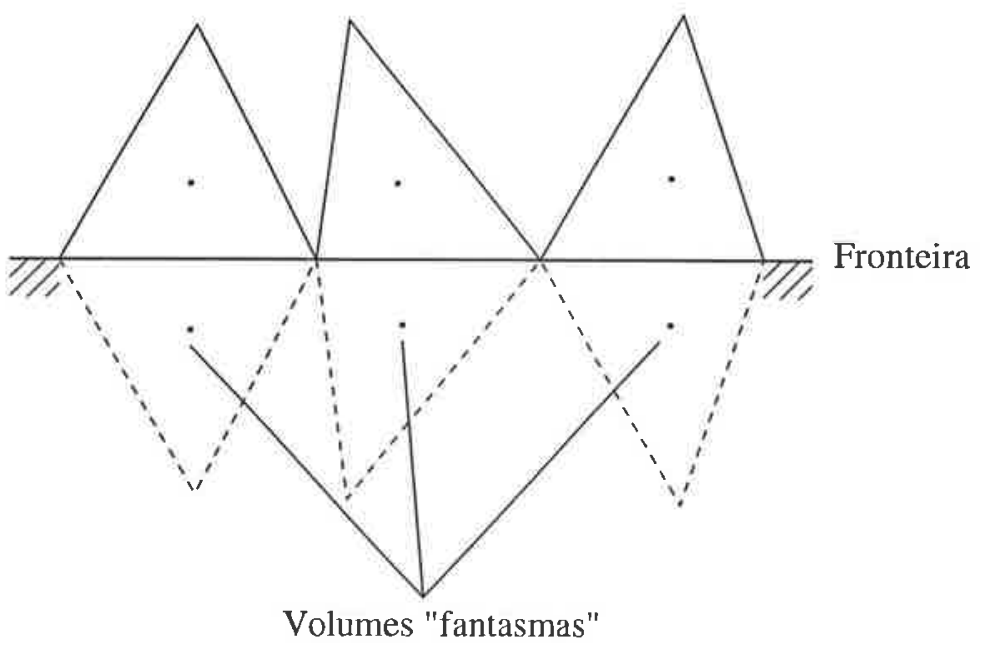

Figura 2.5: Exemplo de construção de volumes "fantasmas"

A figura 2.5 mostra a construção dos volumes "fantasmas" em uma região qualquer da fronteira de um determinado domínio. Os valores das variáveis de 
estado atribuídos a estes volumes definem a condição de contorno aplicada à aresta contida na fronteira do domínio de interesse.

\subsubsection{Parede}

A condição de contorno de parede sólida tem uma particularidade em relação as demais, uma vez que a velocidade do escoamento normal à fronteira é nula, desde que não haja fluxo de massa ou outro fluxo convectivo penetrando na parede sólida. De maneira a analisar a informação transportada pelas características neste tipo de fronteira, considere a equação (2.25), reapresentada abaixo:

$$
\boldsymbol{\Lambda}=\left[\begin{array}{cccc}
k_{x} u+k_{y} v & 0 & 0 & 0 \\
0 & k_{x} u+k_{y} v & 0 & 0 \\
0 & 0 & k_{x} u+k_{y} v+c & 0 \\
0 & 0 & 0 & k_{x} u+k_{y} v-c
\end{array}\right]
$$

Note que duas das quatro características dos escoamentos governados pelas equações de Euler bidimensionais estão relacionadas à autovalores nulos, ou seja, não transmitem qualquer informação através da fronteira. A figura 2.6 apresenta a propagação de características neste tipo de fronteira.

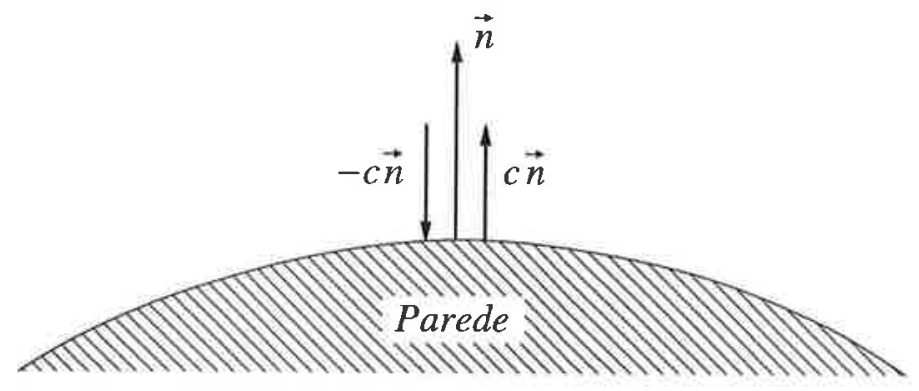

Figura 2.6: Propagação de características em uma parede sólida

Observe que um autovalor é positivo e, portanto, apenas uma condição física pode ser imposta, neste caso é definida pela condição de escorregamento, ou seja, a componente de velocidade normal à superfície deve ser nula $\left(v_{n}=0\right)$. Com a utilização do esquema de Jameson, em que as propriedades nas faces dos elementos são determindas através da média aritmética dos elementos que a cercam, a condição de contorno $v_{n}=0$ implica que a componente normal da velocidade no volume "fantasma" tenha a mesma intensidade da componente de velocidade do volume interno, porém com sentido oposto. Enquanto a componente tangencial é a mesma.

Para entender melhor este fato, considere o sistema de coordenadas gene- 
ralizado apresentado no apêndice A. Admitindo que a velocidade do volume de fronteira interno seja dado pela expressão:

$$
\overrightarrow{\mathbf{v}}=u \hat{\imath}+v \hat{\jmath}
$$

Pode-se, então, escrever as derivadas espaciais no sistema de coordenadas cartesiano de uma propriedade $\phi$ em função das derivadas no sistema generalizado da seguinte maneira:

$$
\begin{aligned}
& \frac{\partial \phi}{\partial x}=\frac{\partial \phi}{\partial \tau} \frac{\partial f^{0}}{\partial x}+\frac{\partial \phi}{\partial \xi} \frac{\partial \xi}{\partial x}+\frac{\partial \phi}{\partial \eta} \frac{\partial \eta}{\partial x}=\xi_{x} \frac{\partial \phi}{\partial \xi}+\eta_{x} \frac{\partial \phi}{\partial \eta} \\
& \frac{\partial \phi}{\partial y}=\frac{\partial \phi}{\partial \tau} \frac{\partial f^{0}}{\partial y}+\frac{\partial \phi}{\partial \xi} \frac{\partial \xi}{\partial y}+\frac{\partial \phi}{\partial \eta} \frac{\partial \eta}{\partial y}=\xi_{y} \frac{\partial \phi}{\partial \xi}+\eta_{y} \frac{\partial \phi}{\partial \eta}
\end{aligned}
$$

Assim, a velocidade do escoamento no volume interno pode ser expressa no sistema generalizado como:

$$
\overrightarrow{\mathbf{v}}=\underbrace{\left(\eta_{x} u+\eta_{y} v\right)}_{v_{n}} \hat{n}+\underbrace{\left(\xi_{x} u+\xi_{y} v\right)}_{v_{t}} \hat{t}
$$

onde $\hat{n}$ e $\hat{t}$ são, respectivamente, os versores "normal" e "tangencial" à superfície no sistema de coordenadas.

Assim, as componentes da velocidade no volume "fantasma", indicadas pelo sobrescrito ghost, são escritas no sistema de coordenadas generalizado da seguinte maneira:

$$
v_{n}^{g h o s t}=-v_{n} \quad, \quad v_{t}^{g h o s t}=v_{t}
$$

E o vetor velocidade neste volume é então escrito como:

$$
\overrightarrow{\mathbf{v}}^{\text {ghost }}=v_{n}^{\text {ghost }} \vec{n}+v_{t}^{\text {ghost }} \vec{t}
$$

Reescrevendo-o, agora, no sistema de coordenadas cartesiano, obtém-se:

$$
\overrightarrow{\mathbf{v}}^{g h o s t}=\left(\eta_{x} v_{n}^{g h o s t}+\xi_{x} v_{t}^{g h o s t}\right) \hat{\imath}+\left(\eta_{y} v_{n}^{g h o s t}+\xi_{y} v_{t}^{g h o s t}\right) \hat{\jmath}
$$

Lembrando que na superfície sólida, $\eta_{x}=k_{x}$ e $\eta_{y}=k_{y}$ e que se a transformação de coordenadas não estiver sujeita a um estiramento anisotrópico, então:

$$
\begin{aligned}
\xi_{x} & =\eta_{y} \\
\xi_{y} & =-\eta_{x}
\end{aligned}
$$


Determina-se facilmente a velocidade do volume "fantasma" nesta condição.

Já o autovalor negativo indica que deve haver uma condição de contorno numérica e para determiná-la é necessário resolver à característica relacionada ao autovalor $(\overrightarrow{\mathbf{v}} \cdot \vec{n}-c)$, ou seja, a quarta equação do sistema (2.31) reescrita abaixo:

$$
\begin{aligned}
\frac{1}{\rho c} \frac{\partial p}{\partial t}-\left(k_{x} \frac{\partial u}{\partial t}+k_{y} \frac{\partial v}{\partial t}\right)= & -\frac{1}{\rho c}\left(u \frac{\partial p}{\partial x}+v \frac{\partial p}{\partial y}\right)+\frac{1}{\rho}\left(k_{x} \frac{\partial p}{\partial x}+k_{y} \frac{\partial p}{\partial y}\right)+ \\
& +\left[\left(k_{x} u-c\right) \frac{\partial u}{\partial x}+k_{y} u \frac{\partial v}{\partial x}\right]+ \\
+ & {\left[\left(k_{y} v-c\right) \frac{\partial v}{\partial y}+k_{x} v \frac{\partial u}{\partial y}\right] }
\end{aligned}
$$

Observe, no entanto, que para cada elemento da fronteira, $k_{x}$ e $k_{y}$ são constantes. Então, pode-se escrever:

$$
k_{x} \frac{\partial u}{\partial t}+k_{y} \frac{\partial v}{\partial t}=\frac{\partial}{\partial t}\left(k_{x} u+k_{y} v\right)=\frac{\partial v_{n}}{\partial t}
$$

onde $v_{n}$ é a velocidade normal à fronteira. Naturalmente, esta derivada corresponde à aceleração centrípeta de uma partícula se movendo em uma direção tangente à parede e pode, portanto, ser relacionadà à velocidade tangencial através da expressão:

$$
\frac{\partial v_{n}}{\partial t}=a_{c}= \pm \frac{v_{t}^{2}}{R_{w}}
$$

em que $v_{t}$ é a velocidade tangencial à fronteira e $R_{w}$ é o raio de curvatura local. Observe que $a_{c}$ corresponde à aceleração centrípeta do fluido numa parede de raio de curvatura $R_{w}$. Assim, o sinal utilizado está relacionado com o formato da parede: caso seja côncava, de modo que a velocidade seja tangencial à parede, o vetor aceleração deve apontar para dentro do domínio (mesmo sentido da normal adotada) e, portanto o sinal é positivo. Por outro lado, se a parede for convexa, o vetor aceleração aponta para fora do domínio (sentido oposto à normal), desta forma o sinal é negativo.

Assim, ao substituir as equações (2.72) e (2.73) em (2.71) obtém-se a seguinte relação característica:

$$
\begin{aligned}
\frac{1}{\rho c} \frac{\partial p}{\partial t} & -\left( \pm \frac{v_{t}^{2}}{R_{w}}\right)+\frac{1}{\rho c}\left(u \frac{\partial p}{\partial x}+v \frac{\partial p}{\partial y}\right)-\frac{1}{\rho}\left(k_{x} \frac{\partial p}{\partial x}+k_{y} \frac{\partial p}{\partial y}\right)+ \\
& -\left[\left(k_{x} v-c\right) \frac{\partial u}{\partial x}+k_{y} u \frac{\partial v}{\partial x}\right]-\left[\left(k_{y} v-c\right) \frac{\partial v}{\partial y}+k_{x} v \frac{\partial u}{\partial y}\right]=0
\end{aligned}
$$

Uma vez que nesta condição de contorno a velocidade normal à fronteira deve ser nula, convém utilizar a transformação de coordenadas apresentada no apên- 
dice $A$, de maneira que o vetor velocidade seja escrito em função das velocidades normal e tangencial à superfície da parede. Assim, a equação característica pode ser escrita como:

$$
\begin{aligned}
\frac{1}{\rho c} \frac{\partial p}{\partial t} & -\left( \pm \frac{v_{t}^{2}}{R_{w}}\right)+\frac{1}{\rho c}[\overbrace{\left(k_{y} u-k_{x} v\right)}^{v_{t}} \frac{\partial p}{\partial \xi}+\overbrace{\left(k_{x} u+k_{y} v\right)}^{v_{n}=0} \frac{\partial p}{\partial \eta}]+ \\
& -\frac{1}{\rho}[\overbrace{\left(k_{x}^{2}+k_{y}^{2}\right)}^{=1} \frac{\partial p}{\partial \eta}]-[u \frac{\partial}{\partial x} \overbrace{\left(k_{x} u+k_{y} v\right)}^{v_{n}=0}-c \frac{\partial u}{\partial x}]+ \\
& -[v \frac{\partial}{\partial y} \overbrace{\left(k_{x} u+k_{y} v\right)}^{v_{n}=0}-c \frac{\partial v}{\partial y}]=0
\end{aligned}
$$

Feitas as devidas simplificações e introduzindo o operador divergente, a equação se resume, apenas, à seguinte expressão:

$$
\frac{1}{\rho c} \frac{\partial p}{\partial t}-\left( \pm \frac{v_{t}^{2}}{R_{w}}\right)+\frac{v_{t}}{\rho c} \frac{\partial p}{\partial \xi}-\frac{1}{\rho} \frac{\partial p}{\partial \eta}+c \nabla \cdot \overrightarrow{\mathbf{v}}=0
$$

Considere, agora, a equação da continuidade em sua forma diferencial conservativa dada pela equação abaixo:

$$
\frac{\partial \rho}{\partial t}+\frac{\partial}{\partial x}(\rho u)+\frac{\partial}{\partial y}(\rho v)=0
$$

Aplicando a regra da cadeia, tem- se:

$$
\frac{\partial \rho}{\partial t}+\rho\left(\frac{\partial u}{\partial x}+\frac{\partial v}{\partial y}\right)+u \frac{\partial \rho}{\partial x}+v \frac{\partial \rho}{\partial y}=0
$$

Multiplicando a equação (2.78) por $c / \rho$ e utilizando a relação isentrópica:

$$
\delta p=c^{2} \delta \rho
$$

Obtém-se:

$$
\frac{1}{\rho c} \frac{\partial p}{\partial t}+c\left(\frac{\partial u}{\partial x}+\frac{\partial v}{\partial y}\right)+\frac{1}{\rho c}\left(u \frac{\partial p}{\partial x}+v \frac{\partial p}{\partial y}\right)=0
$$

Escrevendo, agora, derivadas espaciais em função das coordenadas generalizadas $\xi$ e $\eta$ já apresentadas, chega-se à seguinte expressão:

$$
\frac{1}{\rho c} \frac{\partial p}{\partial t}+c\left(\frac{\partial u}{\partial x}+\frac{\partial v}{\partial y}\right)+\frac{1}{\rho c}\left[u\left(k_{y} \frac{\partial p}{\partial \xi}+k_{x} \frac{\partial p}{\partial \eta}\right)+v\left(-k_{x} \frac{\partial p}{\partial \xi}+k_{y} \frac{\partial p}{\partial \eta}\right)\right]=0
$$

Por fim, separando às componentes normal e tangencial da velocidade (para 
poder utilizar a condição de velocidade normal nula na parede), obtém-se a equação da continuidade na forma:

$$
\frac{1}{\rho c} \frac{\partial p}{\partial t}+c\left(\frac{\partial u}{\partial x}+\frac{\partial v}{\partial y}\right)+\frac{1}{\rho c}[\overbrace{\left(k_{y} u-k_{x} v\right)}^{v_{t}} \frac{\partial p}{\partial \xi}+\overbrace{\left(k_{x} u+k_{y} v\right)}^{v_{n}=0} \frac{\partial p}{\partial \eta}]=0
$$

Finalmente, a equação da continuidade pode ser escrita nesta condição da seguinte maneira:

$$
\frac{1}{\rho c} \frac{\partial p}{\partial t}+c \nabla \cdot \overrightarrow{\mathbf{v}}+\frac{v_{t}}{\rho c} \frac{\partial p}{\partial \xi}=0
$$

Então, subtraindo a equação (2.83) da equação (2.76), obtém-se:

$$
\frac{\partial p}{\partial \eta}= \pm \frac{\rho v_{t}^{2}}{R_{w}}
$$

Assim, só é necessário determinar a pressão na parede para determinar completamente o estado do volume "fantasma" neste tipo de condição de contorno. Entretanto, se $R_{w} \gg 1$ então:

$$
\frac{\partial p}{\partial \eta}=0
$$

Uma discretização fina do contorno de parede, na forma de segmentos de retas torna essa aproximação razoável. Assim, é comum realizar a hipótese de que o gradiente de pressão na parede é desprezível. Desta forma, a pressão estática de um volume "fantasma" é obtida por uma extrapolação de ordem zero desta propriedade com relação ao seu respectivo volume interno. Assim:

$$
p^{g h o s t}=p^{\text {interno }}
$$

\subsubsection{Farfield}

A utilização da formulação característica das equações de Euler certamente não é a forma mais usual para determinar as condições de contorno de farfield em aplicações de escoamentos externos ao redor de aerofólios, já que nestes casos a fronteira está, como o próprio nome sugere, muito distante da parede e, portanto, pode-se simplificar estas condições de contorno, uma vez que as perturbações provenientes da superfície sólida devem chegar muito fracas na fronteira. Geralmente, neste caso são implementadas condições de contorno baseadas na idéia de invariantes de Riemann, que nada mais são que condições de contorno de escoamento potencial completo.

Entretanto, como já foi destacado, ao implementar condições de contorno 
desta forma e, portanto, levar em conta toda a física do modelo matemático do escoamento, espera-se que o código seja mais geral e que possa ser utilizado, inclusive, para resolver escoamentos internos em trabalhos futuros.

O farfield é dividido em duas partes: entrada e saída. Uma vez que o vetor normal utilizado nas equações características (2.31) aponta para dentro do domínio, cada uma destas partes pode ser identificada de acordo com o sinal do produto:

$$
\vec{V} \cdot \vec{n}\left\{\begin{array}{l}
>0 \rightarrow \text { entrada } \\
<0 \rightarrow \text { saída }
\end{array}\right.
$$

O número de condições de controno físicas é uma função da situação do escoamento na fronteira. A tabela 2.1 indica o número condições de contorno físicas e numéricas nas fronteiras de entrada e saída de escoamentos bidimensionais, em que o número total de variáveis dependentes é 4 .

Tabela 2.1: Condições de contorno físicas e numéricas para escoamentos 2-D

\begin{tabular}{ccccc}
\hline \hline & \multicolumn{2}{c}{ Subsônico } & \multicolumn{2}{c}{ Supersônico } \\
\cline { 2 - 5 } & Físicas & Numéricas & Físicas & Numéricas \\
\hline Entrada & 3 & 1 & 4 & 0 \\
Saída & 1 & 3 & 0 & 4 \\
\hline \hline
\end{tabular}

\subsubsection{Entrada}

Viu-se que as considerações feitas anteriormente tem influência direta no número de condições de contorno a serem impostas para problemas bidimensionais de escoamentos invíscidos. Assim, em um ponto qualquer da fronteira, o número de condições de contorno a ser imposto depende da maneira como a informação é transportada ao longo de características que interagem com a fronteira.

A figura 2.7 mostra a propagação de características em fronteiras de entrada tanto subsônicas quanto supersônicas. Observa-se que as características $(\overrightarrow{\mathbf{v}} \cdot \vec{n})$, que tem multiplicidade 2 , e $(\overrightarrow{\mathbf{v}} \cdot \vec{n}+c)$ são sempre positivas. Portanto, elas sempre transportam informação da fronteira para o domínio do escoamento. Isto requer que os valores das quantidades transportadas sejam impostos neste ponto para cada característica que entra no domínio através de três condições de contorno físicas. A quarta característica, no entanto, $(\overrightarrow{\mathbf{v}} \cdot \vec{n}-c)$ é negativa para escoamentos subsônicos e positiva em escoamentos supersônicos. Assim, no primeiro caso, deve-se deixar este grau de liberdade livre, utilizando para isto uma condição de contorno numérica, enquanto no último, deve se fixar mais uma propriedade do 
escoamento não-perturbado.

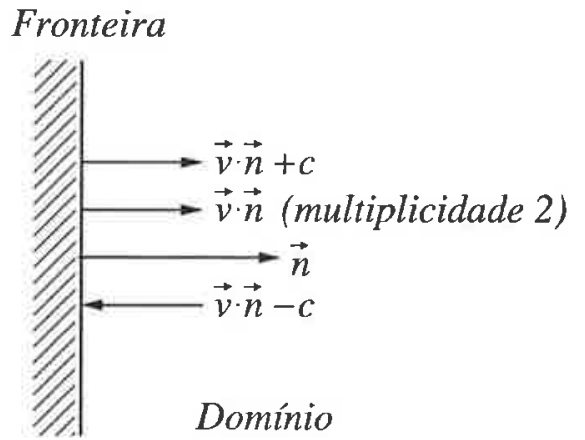

(a) Caso subsônico

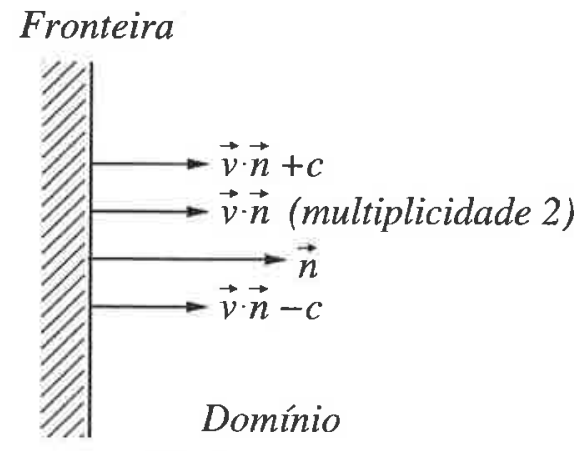

(b) Caso supersônico

Figura 2.7: Propagação de características em fronteiras de entrada

Subsônica Nesta condição, três características entram no domínio enquanto uma sai. Portanto, para determinar o estado dos volumes "fantasmas" nesta região, basta fixar 3 propriedades do escoamento não-perturbado e resolver a característica associada a $\vec{v}-c$, ou seja, a última equação do sistema (2.31) reescrita abaixo:

$$
\begin{gathered}
\frac{\partial p}{\partial t}-\rho c\left(k_{x} \frac{\partial u}{\partial t}+k_{y} \frac{\partial v}{\partial t}\right)=-\left(u-k_{x} c\right) \frac{\partial p}{\partial x}-\left(v-k_{y} c\right) \frac{\partial p}{\partial y}+ \\
+\rho c\left[\left(k_{x} u-c\right) \frac{\partial u}{\partial x}+k_{y} u \frac{\partial v}{\partial x}\right]+\rho c\left[\left(k_{y} v-c\right) \frac{\partial v}{\partial y}+k_{x} v \frac{\partial u}{\partial y}\right]=R_{4}
\end{gathered}
$$

A questão que vem à tona é sobre quais propriedades se deve fixar. Hirsch (2007) mostra que todas as propriedades de estagnação são constantes ao longo de linhas de corrente, mas que os valores podem ser diferentes de uma para outra. Entretanto, se o escoamento que entra no domínio é uniforme, então existe apenas um valor constante no campo do escoamento.

Assim, duas variáveis termodinâmicas são geralmente determinadas pelas condições de estagnação a montante. Usualmente, pressão e temperatura de estagnação podem ser impostas. Observe, no entanto, que entropia e entalpia de estagnação poderiam ser fixadas de modo equivalente. A terceira variável física é definida pelas componentes da velocidade (no caso particular de aerofólios, o ângulo de incidência).

Assumindo que:

$$
\delta p=\frac{\partial p}{\partial U} \delta U \Rightarrow \delta p-\frac{\partial p}{\partial U} \delta U=0
$$

onde $\delta$ é tal que, aplicada à uma variável genérica $z$, resulta em:

$$
\delta z=\Delta t \frac{\partial z}{\partial t}=z_{\text {ghost }}^{n+1}-z_{\text {ghost }}^{n} \quad \text { e } \quad U=\sqrt{u^{2}+v^{2}}
$$


Pode se reescrever a equação (2.88) conforme segue:

$$
\delta p-\rho c\left(k_{x} \delta u+k_{y} \delta v\right)=\Delta t R_{4}
$$

Mas, se o ângulo de ataque $(\alpha)$ é fixo, então as variações das componentes do vetor velocidade provenientes de perturbações vindas da superfície do aerofólio devem permanecer proporcionais:

$$
\frac{\delta u}{\delta v}=\frac{u}{v} \Rightarrow \delta v=\frac{v \delta u}{u}
$$

Substituindo as equações (2.89) e (2.92) na equação característica (2.91), obtém-se:

$$
\frac{\partial p}{\partial U} \delta U-\rho c \delta u\left(k_{x}+k_{y} \frac{v}{u}\right)=\Delta t R_{4}
$$

Mas, se $U=\sqrt{u^{2}+v^{2}}$, então:

$$
\delta U=\frac{1}{2 U}(2 u \delta u+2 v \delta v) \Rightarrow \delta U=\frac{u \delta u}{U}+\frac{v \delta v}{U}
$$

Substituindo (2.92) em (2.94), tem-se:

$$
\delta U=\frac{u \delta u}{U}+\frac{v}{U} \frac{v \delta u}{u} \Rightarrow U \delta U=\delta u\left(u+v \frac{v}{u}\right) \Rightarrow \delta U=\frac{\delta u}{U}\left(u+v \frac{v}{u}\right)
$$

E, substituindo (2.95) em (2.93) é possível escrever $\delta u$ da seguinte forma:

$$
\delta u=\frac{\Delta t R_{4}}{\left\{\left[\frac{\partial p}{\partial U}\left(\frac{u}{U}+\frac{v^{2}}{u U}\right)\right]-\rho c\left(k_{x}+k_{y} \frac{v}{u}\right)\right\}}
$$

Lembrando, ainda, que:

$$
p=p_{o}\left[1+\frac{(\gamma-1)}{2} M^{2}\right]^{-\gamma /(\gamma-1)}
$$

Então, pode-se escrever:

$$
\frac{\partial p}{\partial U}=-\frac{p_{o} \gamma}{(\gamma-1)}\left[1+\frac{(\gamma-1)}{2} M^{2}\right]^{\frac{-\gamma}{\gamma-1}-1} 2 \frac{(\gamma-1)}{2} M \frac{\partial M}{\partial U}
$$

Mas, $M=\frac{U}{c} \Rightarrow \frac{\partial M}{\partial U}=\frac{1}{c}$, que pode ser aplicado à equação acima, resultando na seguinte expressão:

$$
\frac{\partial p}{\partial U}=-\frac{p_{o} \gamma M}{c}\left[1+\frac{(\gamma-1)}{2} M^{2}\right]^{\frac{1-2 \gamma}{\gamma-1}}
$$

Substituindo (2.99) em (2.96), pode-se obter $\delta u$ e, conseqüentemente, $\delta v$ atra- 
vés da expressão (2.92). Assim, as componentes da velocidade são dadas por:

$$
\begin{aligned}
& u_{\text {ghost }}^{n+1}=u_{\text {ghost }}^{n}+\delta u \\
& v_{\text {ghost }}^{n+1}=v_{\text {ghost }}^{n}+\delta v
\end{aligned}
$$

Lembrando que as propriedades de estagnação fixadas podem ser determinadas através das expressões:

$$
\begin{aligned}
& p_{o}=p\left[1-\frac{\gamma-1}{\gamma+1} \frac{\left(u^{2}+v^{2}\right)}{a_{*}^{2}}\right]^{-\gamma / \gamma-1} \\
& T_{o}=T\left[1-\frac{\gamma-1}{\gamma+1} \frac{\left(u^{2}+v^{2}\right)}{a_{*}^{2}}\right]^{-1}
\end{aligned}
$$

onde a velocidade do som crítica ${ }^{5}\left(a_{*}\right)$ é dada por:

$$
a_{*}^{2}=\frac{2 \gamma T}{\gamma+1}
$$

Então, as propriedades restantes para completar o estado dos volumes "fantasmas" são funções apenas da velocidade:

$$
\begin{gathered}
p=p(u) \Rightarrow p_{\text {ghost }}^{n+1}=p\left(u_{\text {ghost }}^{n+1}\right) \\
T=T(u) \Rightarrow T_{\text {ghost }}^{n+1}=T\left(u_{\text {ghost }}^{n+1}\right)
\end{gathered}
$$

Supersônica Na condição de entrada supersônica, as 4 características entram no domínio e, portanto, toda a informação é transportada da condição de escoamento não-perturbado. Assim, todas as condições de contorno são físicas e, conseqüentemente, todas as propriedades são fixadas:

$$
\begin{aligned}
& u_{\text {ghost }}^{n+1}=u_{\text {ghost }}^{n}=\cdots=u_{\text {ghost }}^{0} \\
& v_{\text {ghost }}^{n+1}=v_{\text {ghost }}^{n}=\cdots=v_{\text {ghost }}^{0} \\
& p_{\text {ghost }}^{n+1}=p_{\text {ghost }}^{n}=\cdots=p_{\text {ghost }}^{0} \\
& \rho_{\text {ghost }}^{n+1}=\rho_{\text {ghost }}^{n}=\cdots=\rho_{\text {ghost }}^{0}
\end{aligned}
$$

\subsubsection{Saida}

Considerações similares à entrada podem ser feitas para a saída. Três características $\overrightarrow{\mathbf{v}} \cdot \vec{n}$ (multiplicidade 2) e $(\overrightarrow{\mathbf{v}} \cdot \vec{n}-c)$ são sempre negativas e sempre alcançam a fronteira de dentro do domínio e portanto determinam 3 das 4 variáveis características do plano de saída a partir do comportamento do escoamento

\footnotetext{
${ }^{5}$ velocidade do som quando o número de Mach é igual a 1.
} 
interior. A $4^{\mathrm{a}}$ condição depende do número de Mach. Para saídas supersônicas nenhuma condição de contorno deve ser imposta, enquanto que para saídas subsônicas, uma condição de contorno deve ser fixa. A figura 2.8 mostra a propagação de características em fronteiras de saída.

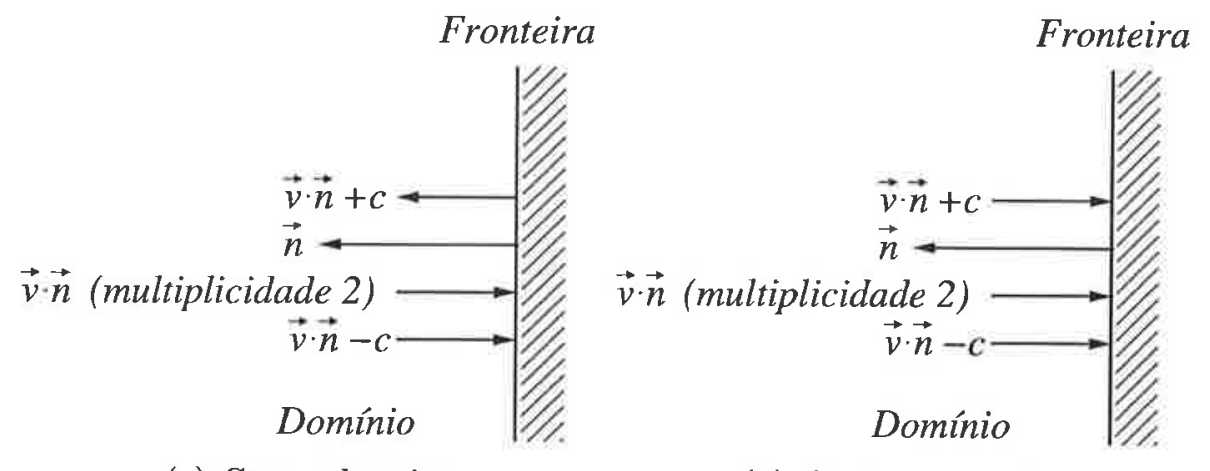

(a) Caso subsônico

(b) Caso supersônico

Figura 2.8: Propagação de características em fronteiras de saída

Subsônica Neste caso, três características saem do domínio e apenas uma entra $(\overrightarrow{\mathbf{v}} \cdot \vec{n}+c)$. Portanto, três condiçôes de contorno devem ser calculadas, enquanto a quarta condição, associada ao autovalor positivo $(\overrightarrow{\mathbf{v}} \cdot \vec{n}+c)$, propaga informação da fronteira para a região do escoamento e está, conseqüentemente, associada com uma condição de contorno física, deve ser fixada. A condição física mais apropriada, particularmente para escoamentos internos e correspondente a maioria das situações experimentais, consiste em fixar a pressão estática de saída. Isto também pode ser aplicado em problemas de escoamento externo (HIRSCH, 1988b).

Ao se fixar a pressão estática $(\delta p=0)$ e excluir a característica associada ao autovalor $(\overrightarrow{\mathbf{v}} \cdot \vec{n}+c)$, que entra no domínio, o sistema (2.31) se resume à seguinte forma:

$$
\left\{\begin{aligned}
\delta \rho & =\Delta t R_{1} \\
k_{y} \delta u-k_{x} \delta v & =\Delta t R_{2} \\
-\rho c\left(k_{x} \delta u+k_{y} \delta v\right) & =\Delta t R_{4}
\end{aligned}\right.
$$

A primeira equação do sistema (2.103) fornece diretamente a variação da massa específica no volume fantasma, para encontrar as demais propriedades, basta resolver o sistema abaixo, formado pelas características restantes.

$$
\begin{cases}k_{y} \delta u-k_{x} \delta v & =\Delta t R_{2} \\ -\rho c\left(k_{x} \delta u+k_{y} \delta v\right) & =\Delta t R_{4}\end{cases}
$$


Caso $k_{y} \neq 0$, da primeira equação, tem-se:

$$
\delta u=\frac{\Delta t R_{2}+k_{x} \delta v}{k_{y}}
$$

E, substituindo a equação (2.105) na segunda equação do sistema (2.104), obtém-se:

$$
\delta v=-\left(k_{x} R_{2}+\frac{k_{y} R_{4}}{\rho c}\right) \Delta t
$$

Mas, se $k_{y}=0$ e, consequentemente, $k_{x}=-1$, deve-se fazer:

$$
\delta v=\Delta t R_{2} \quad \mathrm{e} \quad \delta u=\frac{\Delta t R_{4}}{\rho c}
$$

Assim,

$$
\begin{aligned}
& \rho_{\text {ghost }}^{n+1}=\rho_{\text {ghost }}^{n}+\delta \rho \\
& u_{\text {ghost }}^{n+1}=u_{\text {ghost }}^{n}+\delta u \\
& v_{\text {ghost }}^{n+1}=v_{\text {ghost }}^{n}+\delta v
\end{aligned}
$$

Supersônica Nesta condição de saída, todos os autovalores são negativos e nenhuma condição física deve ser especificada. Todas as variáveis de contorno são definidas pelo escoamento interior, via equações características (2.31). Assim, basta resolver este sistema de equações e determinar as variáveis primitivas que definem o estado do volume "fantasma". Isto, no entanto, pode ser feito de diversas maneiras, aqui, apresenta-se apenas uma sugestão de como resolvê-lo:

Inicialmente, ao somar as duas últimas equações do sistema (2.31), obtém-se uma expressão direta para atualização da pressão estática dada por:

$$
\frac{\partial p}{\partial t}=\frac{R_{3}+R_{4}}{2} \Rightarrow \delta p=\left(\frac{R_{3}+R_{4}}{2}\right) \Delta t
$$

Da primeira equação do sistema (2.31), tem-se:

$$
\delta \rho=\Delta t R_{1}+\frac{\delta p}{c^{2}}
$$

As duas últimas informações que restam para definir o estado do volume "fantasma" podem ser obtidos do sistema formado por estas duas equações:

$$
\left\{\begin{array}{lll}
k_{y} \frac{\partial u}{\partial t}-k_{x} \frac{\partial v}{\partial t} & =R_{2} \\
\frac{\partial p}{\partial t}-\rho c\left(k_{x} \frac{\partial u}{\partial t}+k_{y} \frac{\partial v}{\partial t}\right) & =R_{4}
\end{array}\right.
$$


Caso $k_{y} \neq 0$, da primeira equação, tem-se:

$$
\delta u=\frac{\Delta t R_{2}+k_{x} \delta v}{k_{y}}
$$

Substituindo as equações (2.108) e (2.111) na segunda equação do sistema (2.110), obtém-se:

$$
\delta v=\frac{k_{y}}{\rho c}\left(\delta p-\Delta t R_{4}\right)-k_{x} \Delta t R_{2}
$$

Mas, se $k_{y}=0$ e, portanto $k_{x}=-1$, deve-se fazer:

$$
\delta v=\Delta t R_{2} \quad \text { e } \quad \delta u=\frac{\Delta t R_{4}-\delta p}{\rho c}
$$

Assim,

$$
\begin{aligned}
& \rho_{\text {ghost }}^{n+1}=\rho_{\text {ghost }}^{n}+\delta \rho \\
& u_{\text {ghost }}^{n+1}=u_{\text {ghost }}^{n}+\delta u \\
& v_{\text {ghost }}^{n+1}=v_{\text {ghost }}^{n}+\delta v \\
& p_{\text {ghost }}^{n+1}=p_{\text {ghost }}^{n}+\delta p
\end{aligned}
$$

\subsection{Validação}

A validação do código computacional implementado para a resolução de escoamentos foi realizada por comparação ao programa comercial já consagrado no mercado, o CFD ++. Seis casos de validação com características bem particulares foram simulados de maneira a testar sua faixa de aplicação e robustez. A tabela 2.2 mostra os perfis e condições de escoamento não-perturbado de cada um destes.

Tabela 2.2: Casos de validação do solver do escoamento

\begin{tabular}{cccccc}
\hline \hline Caso & Aerofólio & $M_{\infty}$ & $\alpha\left(^{\circ}\right)$ & $p_{\infty}(\mathrm{Pa})$ & $T_{\infty}(\mathrm{K})$ \\
\hline 1 & NACA 0012 & 0.3 & 0.0 & 101325.0 & 288.15 \\
2 & RAE 2822 & 0.3 & 0.0 & 101325.0 & 288.15 \\
3 & Selig 1223 & 0.1 & 8.0 & 101325.0 & 288.15 \\
4 & NACA 0012 & 0.8 & 0.0 & 101325.0 & 288.15 \\
5 & RAE 2822 & 0.8 & 0.0 & 101325.0 & 288.15 \\
6 & RAE 2822 & 0.75 & 1.0 & 101325.0 & 288.15 \\
\hline \hline
\end{tabular}

Os casos 1 à 3 apresentados pelas figuras 2.9, 2.10 e 2.11 representam escoamentos subsônicos sobre os perfis NACA 0012, RAE 2822 e Selig 1223, respectivamente. Estas figuras mostram a robustez do método ao simular escoamentos de 
baixa velocidade, inclusive, um caso incompressível, como o escoamento à número de Mach 0.1 (Caso 3).

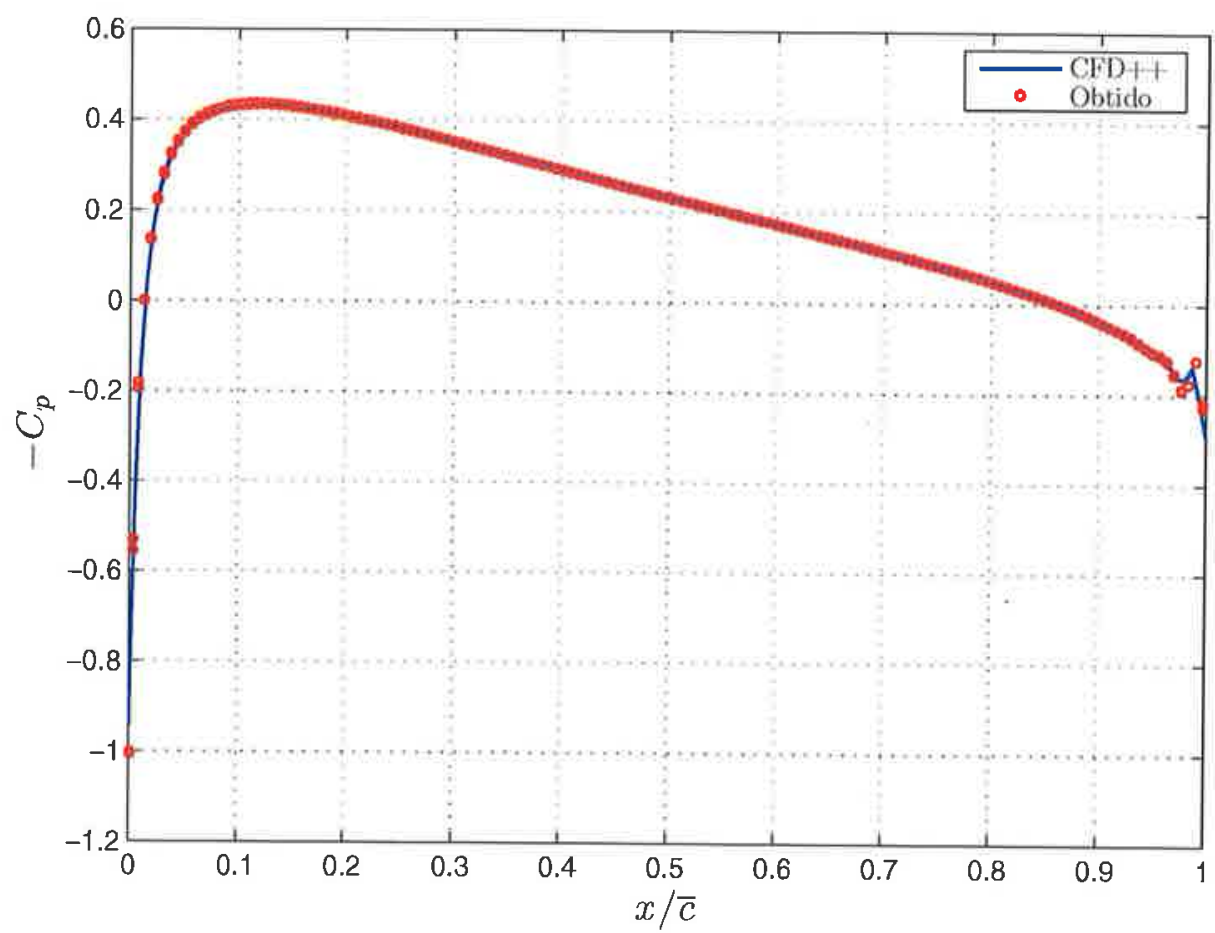

Figura 2.9: Caso 1 - Perfil NACA $0012\left(M_{\infty}=0.3, \alpha=0.0^{\circ}\right)$

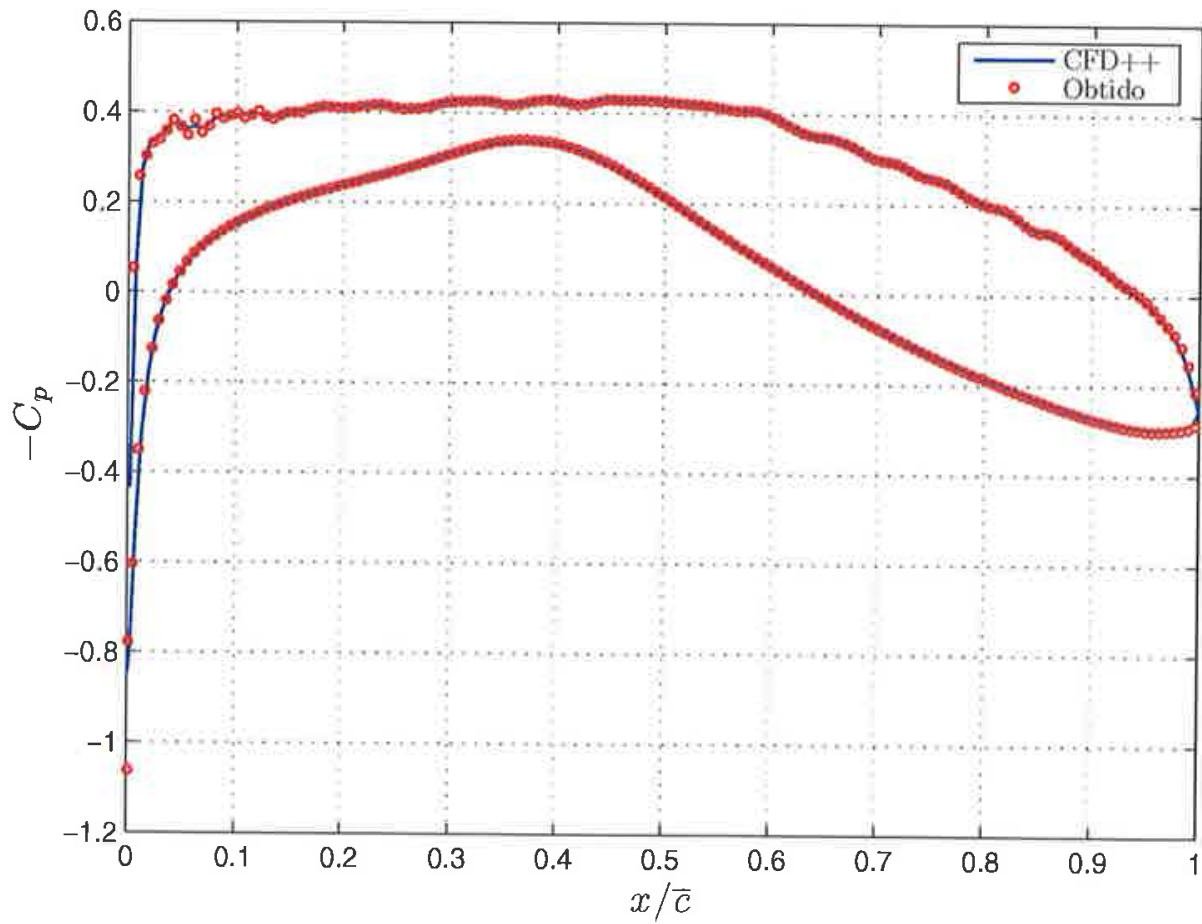

Figura 2.10: Caso 2 - Perfil RAE $2822\left(M_{\infty}=0.3, \alpha=0.0^{\circ}\right)$

Como já dito na seção 2.2, a adimensionalização utilizada não é adequada à escoamentos externos incompressíveis. Apesar dos bons resultados obtidos com- 
parados ao $\mathrm{CFD}++$, o reflexo desta característica do método numérico empregado refletiu na taxa de convergência dos resultados. Verifica-se que os casos de número de Mach baixos $\left(M_{\infty}<0.3\right)$ necessitam de mais tempo de simulação para convergir do que àqueles em regime de escoamento transônicos, que são as aplicações de maior interesse neste trabalho e na indústria aeronáutica, uma vez que a maioria das aeronaves comerciais tem velocidade de cruzeiro nestas condições de escoamento.

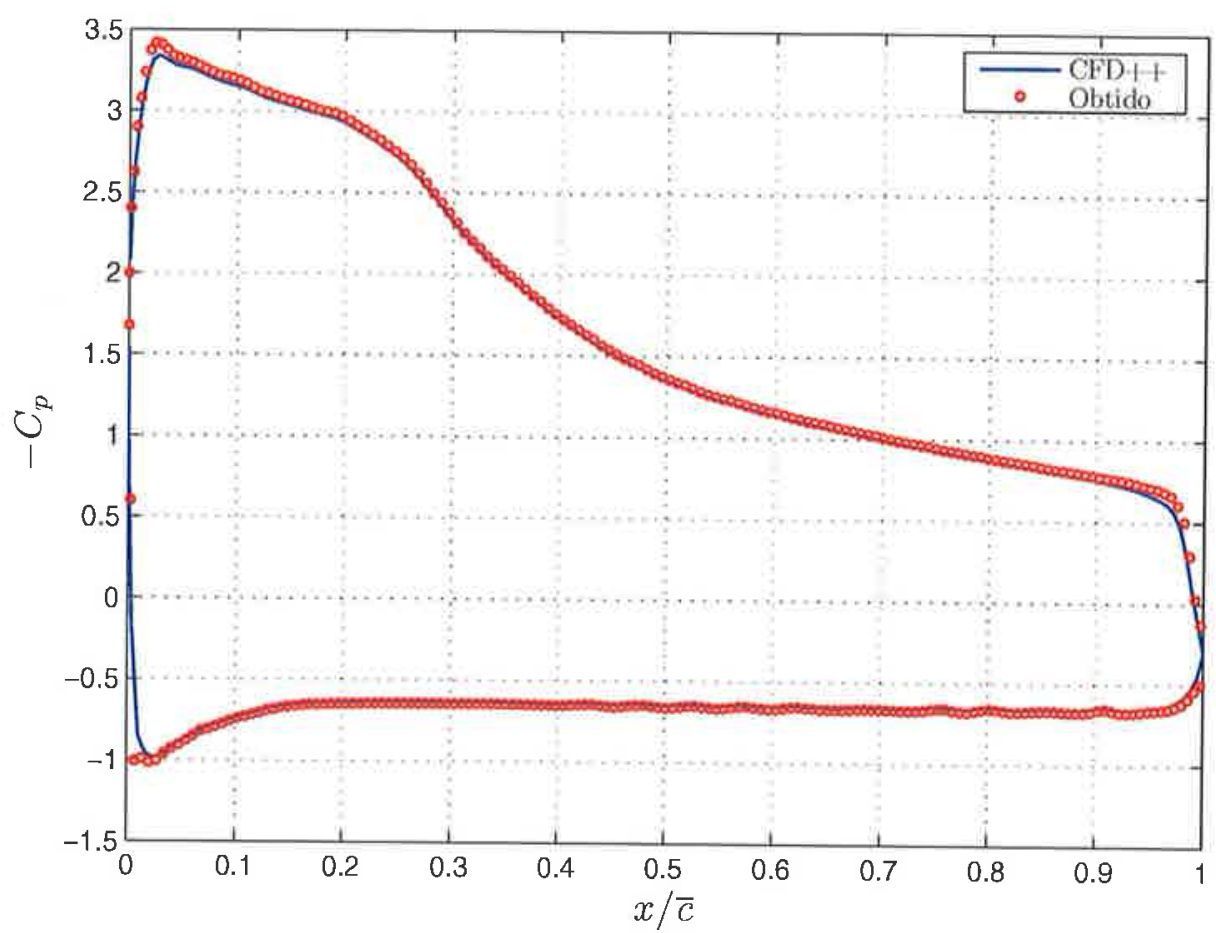

Figura 2.11: Caso 3 - Perfil Selig $1223\left(M_{\infty}=0.1, \alpha=8.0^{\circ}\right)$

Os casos 4- 6 representam simulações em regime de escoamento transônico ao redor dos perfis NACA 0012 e RAE2822. Nestes casos é possível verificar a qualidade dos resultados obtidos ao tratar as ondas de choque. Observa-se que a dissipação artificial adicionada está adequada às aplicações de interesse, uma vez que as ondas de choque encontradas em todas estas simulações são bem definidas em poucos pontos. É importante lembrar que programas comerciais freqüentemente fazem uso de dissipação numérica excessiva para tornar seus códigos mais robustos, capazes de convergir para uma gama maior de problemas. Entretanto este excesso de dissipação pode levar a degradação da solução obtida (AZEVEDO, 2006).

Observa-se, ainda, uma oscilação da distribuição de pressão no extradorso dos casos 2, 5 e 6, que correspondem à escoamentos sobre o perfil RAE2822. Entretanto esta oscilação ocorreu em ambos os códigos (o desenvolvido neste 


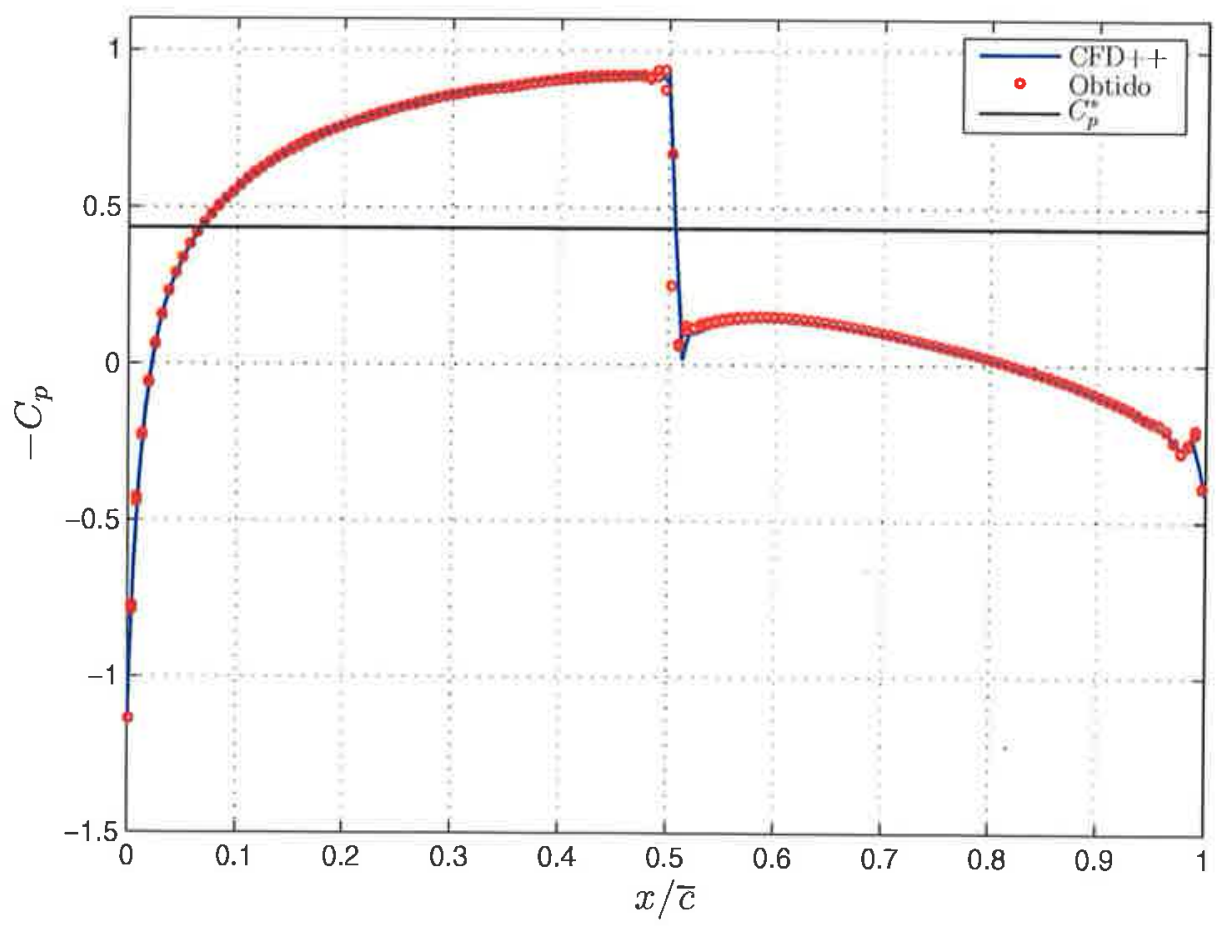

Figura 2.12: Caso 4 - Perfil NACA $0012\left(M_{\infty}=0.8, \alpha=0.0^{\circ}\right)$

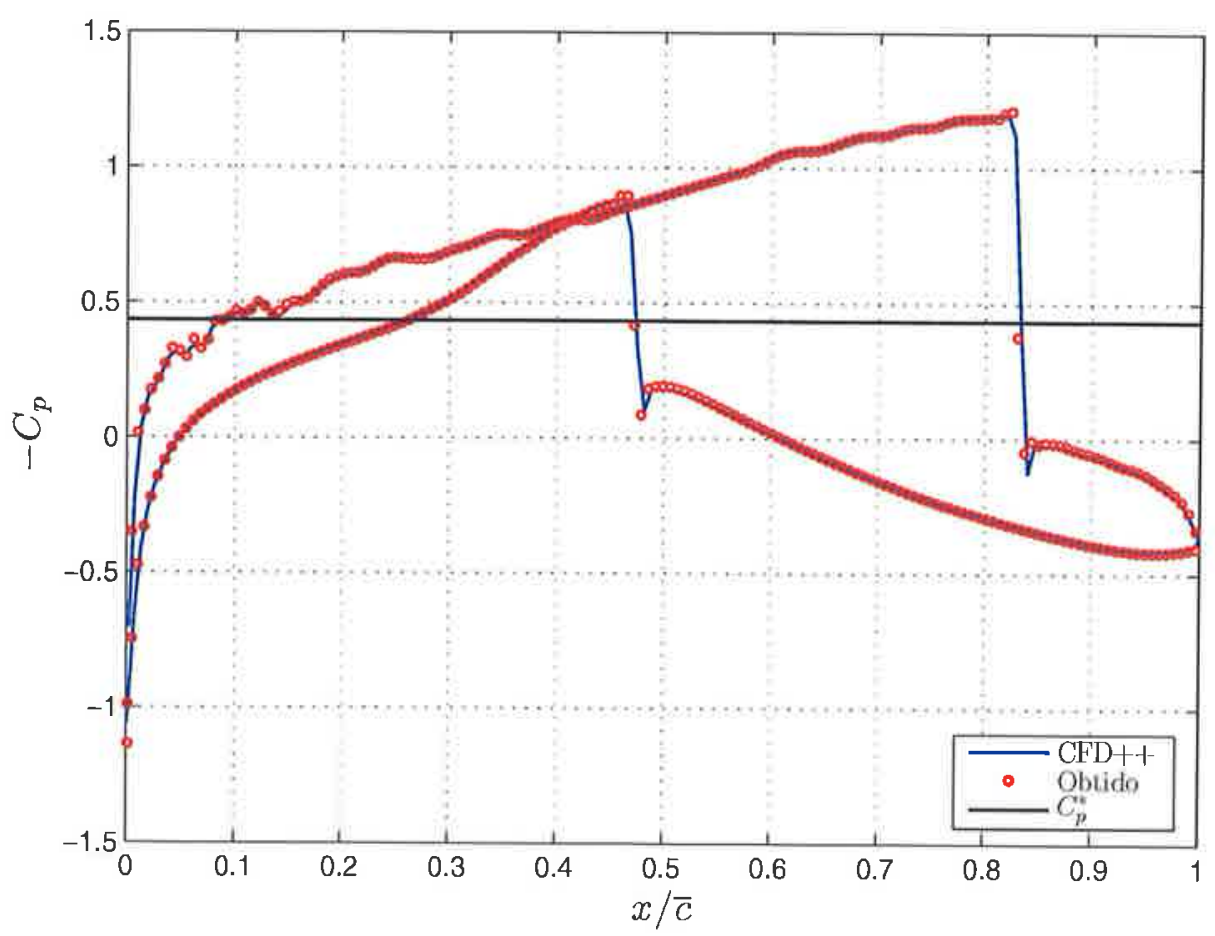

Figura 2.13: Caso 5 - Perfil RAE $2822\left(M_{\infty}=0.8, \alpha=0.0^{\circ}\right)$

trabalho e o $\mathrm{CFD}++$ ), o que pode indicar que o problema está relacionado com a geometria ou a malha utilizada.

A título de ilustração, a figura 2.15 mostra os campos de Mach, pressão, 


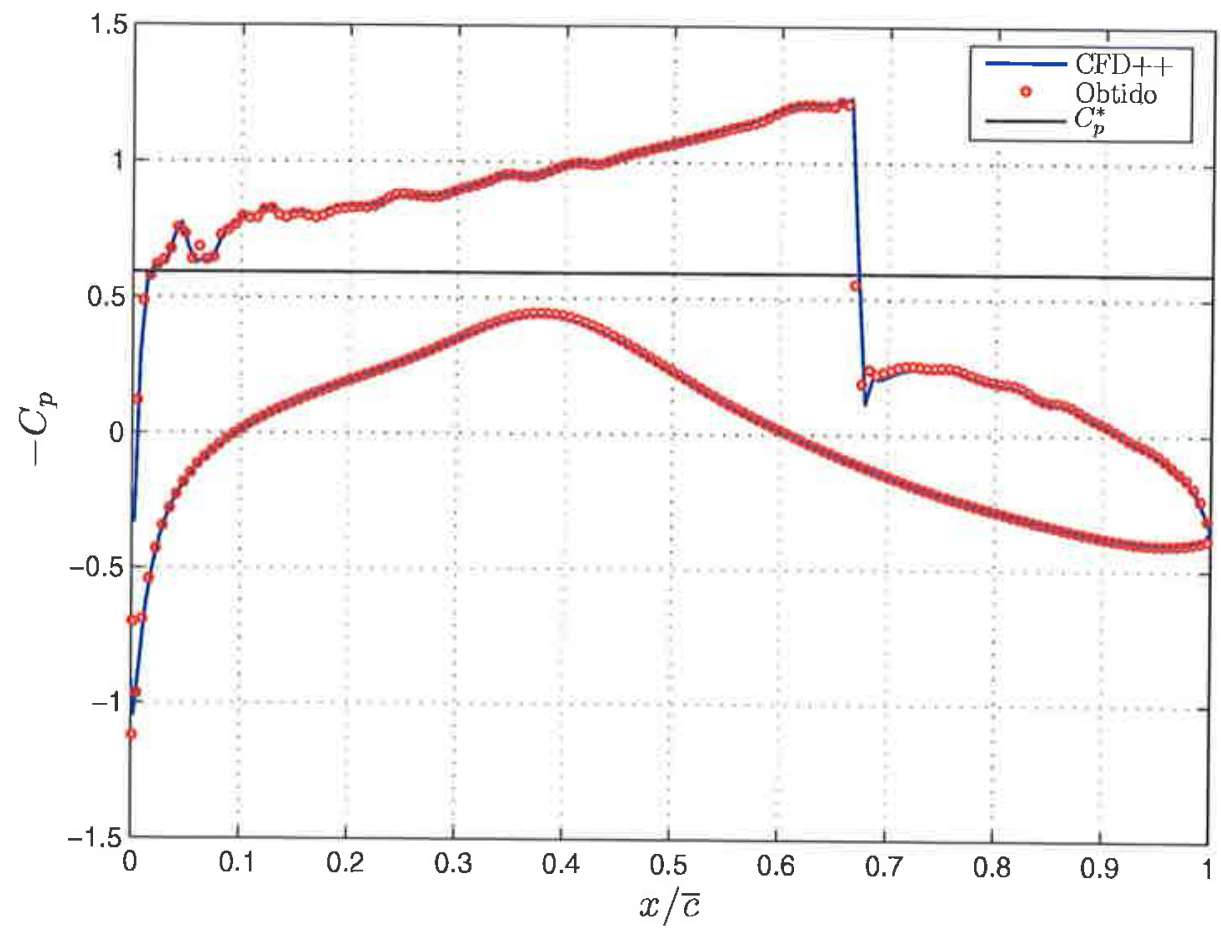

Figura 2.14: Caso 6 - Perfil RAE $2822\left(M_{\infty}=0.75, \alpha=1.0^{\circ}\right)$

temperatura e de um termo proporcional à variação de entropia do caso 6 para dar ao leitor uma idéia da solução no domínio computacional.

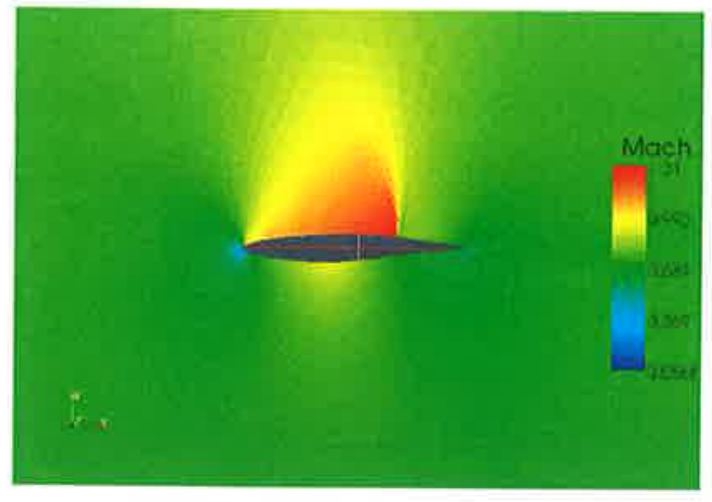

(a) Número de Mach

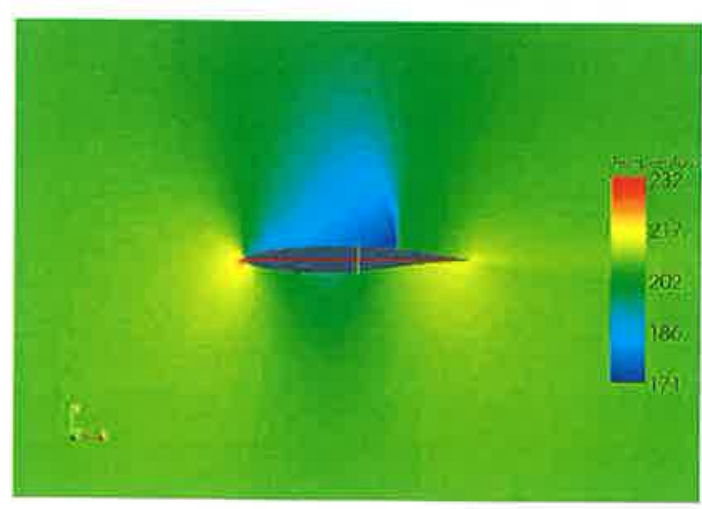

(c) Temperatura $(\mathrm{em} K)$

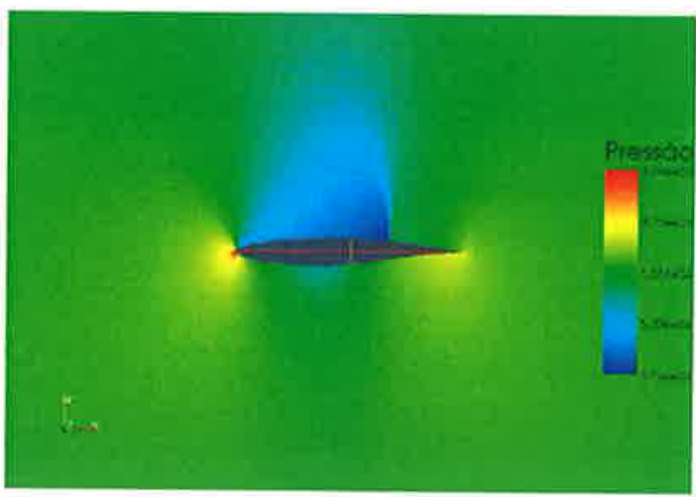

(b) Pressão estática (em $P a$ )

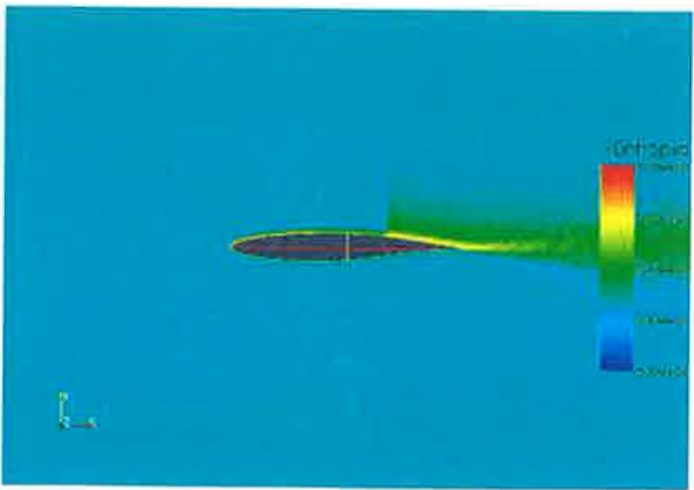

(d) $p / \rho^{\gamma}$

Figura 2.15: Campo de solução do Caso $6\left(M_{\infty}=0.75, \alpha=1.0^{\circ}\right)$ 
A figura 2.15(d) indica regiões onde há geração de entropia através de um termo $p / \rho^{\gamma}$, que é proporcional à ela. Uma vez que a pressão de estagnação $p_{0}$ não permanece constante ao atravessar a onda de choque, as relações invíscidas de choque implicam em uma variação descontínua de entropia através dele (HIRSCH, 2007). Assim, a onda de choque, naturalmente, provocaria geração de entropia. Entretanto, observa-se que na região imediatamente adjacente a superfície do aerofólio, também, existe uma camada de elementos onde também há geração de entropia. Este fato pode ser justificado pela dissipação artificial adicionada pelo método numérico para a resolução das equações de Euler em regiões onde o gradiente de pressão é elevado, o que possibilita a geração numérica de entropia.

A figura 2.16 ainda mostra a malha utilizada neste mesmo caso. O procedimento de geração de malha, realizado pelo software comercial Gambit com a triangulação de Delaunay, é o mesmo descrito por Ceze (2008) e foi realizado para discretizar o domínio de todos os casos presentes neste trabalho.

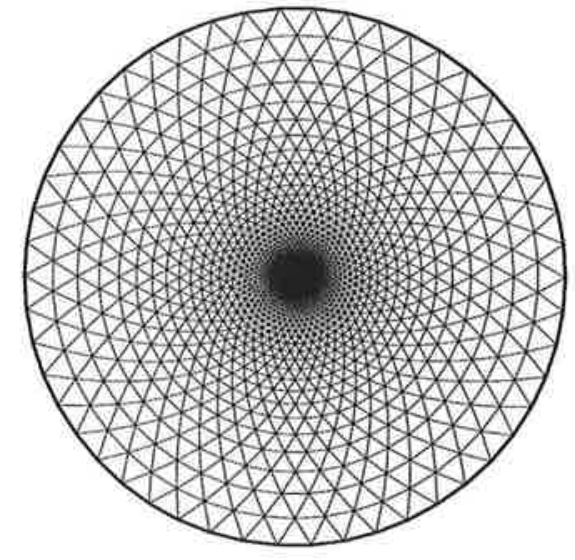

(a) Geral

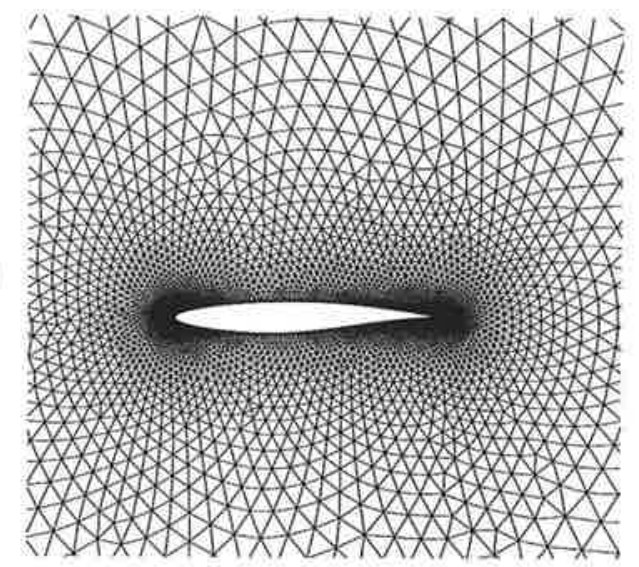

(b) Detalhe

Figura 2.16: Malha utilizada

Assim, o código implementado é considerado validado para os objetivos propostos, mostrando que os resultados obtidos com o tratamento das condições de contorno utilizado (embora não seja estritamente necessário para as aplicações aqui apresentadas) convergem para os mesmos obtidos com o CFD ++ e com o código desenvolvido por Ceze (2008), que simulou os mesmos casos de validação com condições de contorno de invariantes de Riemann, descritos na literatura para implementação das condições de farfield. 


\section{Teoria de Controle APlicada Às EquaÇões DE EULER}

A implementação numérica do método adjunto requer a utilização de uma forma discretizada da equação adjunta. Existem, basicamente, duas formas possíveis para sua obtenção. Primeiro, ela pode ser derivada à partir da forma discretizada das equações que governam o escoamento e, portanto, seria obtida diretamente em uma forma discretizada. Esta forma, conhecida na literatura como formulação discreta do método, fica, no entanto, condicionada à forma como as equações da mecânica dos fluidos foram discretizadas, tanto no que diz respeito à complexidade, quanto à precisão dos resultados. Este procedimento ainda pode levar a expressões bastante complexas, especialmente quando se usam aproximações de ordem superior para as equações da mecânica dos fluidos.

Alternativamente, a equação adjunta pode ser derivada a partir da forma original das equações que governam o escoamento para, só então, ser discretizada. Esta é a formulação contínua do método. A equação adjunta pode ser então resolvida de modo independente dos métodos utilizados para obter a solução das equações do escoamento. Deve-se ressaltar que a forma discretizada da equação adjunta obtida na formulação contínua não coincide com aquela da formulação discreta. Pode-se atribuir esta diferença ao fato de que, nesta última, a restrição que se está impondo corresponde a uma particular discretização das equações da mecânica dos fluidos, ao invés das equações originais.

Em princípio, se a discretização das equações da mecânica dos fluidos é consistente, de modo que seus resultados aproximam a solução exata à medida que a malha computacional é refinada, então os resultados da formulação discreta do método adjunto também devem se aproximar àqueles da formulação contínua com o refinamento da malha, uma vez que eles impõe precisamente essa aproximação consistente do escoamento.

Jameson e Nadarajah (2000) comparam ambas as formulações do método ad- 
junto e concluem que, no procedimento utilizado por eles, os gradientes adjuntos discretos são mais condizentes com os gradientes obtidos por diferenças finitas do que os gradientes contínuos, mas as diferenças são geralmente pequenas. Entretanto, a convergência da função de mérito não é afetada significativamente quando se utiliza o gradiente discreto ao invés do contínuo. Conseqüentemente, não se encontra um benefício particular em utilizar o método adjunto discreto, que requer maior custo computacional.

A finalidade deste trabalho é realizar um estudo teórico aprofundado do método adjunto, com base na aplicação de escoamento Euler 2-D em torno de aerofólios. Principalmente no que diz respeito ao desenvolvimento de condições de contorno apropriadas, de maneira a garantir que o problema adjunto seja sempre tão bem-posto quanto o problema original (as equações que governam o escoamento). Para isso, pretende-se adotar a formulação contínua do método, que se associa diretamente às equações da mecânica dos fluidos. Sua independência com respeito ao método numérico adotado para a simulação do escoamento traz vantagens importantes: permite uma abordagem conceitual mais genérica das equações adjuntas e das suas condições de contorno. Uma rotina assim construída pode, cm princípio, ser associada a diferentes códigos de simulação do escoamento, contanto que as propriedades fixadas sejam as mesmas propostas no código que resolve as equações que governam o escoamento.

\subsection{As Equações Adjuntas}

O principal objetivo de métodos de otimização consiste em minimizar a função que representa uma determinada medida de mérito. Considere, para a derivação das equações adjuntas, uma medida de mérito geral dada por:

$$
I=\int_{B_{w}} g(\mathbf{V}) \mathrm{d} s
$$

onde $g(\mathbf{V})$ representa uma função escalar genérica em função do vetor de variáveis primitivas $\mathrm{V}$, definido pela primeira relação de $(2.16)$ e d $s$ é o elemento de comprimento de arco infinitesimal sobre o aerofólio. A integral é feita sobre toda a superfície do aerofólio $\left(B_{w}\right)$ com o objetivo de encontrar a geometria que minimiza o funcional $I$.

Com o auxílio da notação indicial e a utilização de um sistema de coordenadas generalizado apresentado no apêndice A, pode-se determinar sua derivada com relação ao vetor de variáveis de estado (Q), aplicando a regra da cadeia, da 
seguinte maneira:

$$
\frac{\partial I}{\partial \mathbf{Q}}=\int_{B_{w}} \frac{\partial g}{\partial V_{\alpha}} \frac{\partial V_{\alpha}}{\partial Q_{\beta}} \mathrm{d} s=\int_{B_{w}} \frac{\partial g}{\partial Q_{\beta}} \mathrm{d} s=\int_{B_{w}} \frac{\partial g}{\partial \mathbf{Q}} \frac{\mathrm{d} s}{\mathrm{~d} \xi} \mathrm{d} \xi
$$

Neste caso, de maneira a melhorar o entendimento dos índices da notação, adota-se a seguinte convenção: letras gregas representam o espaço de fase, ou seja, estão relacionados com o número de equações do sistema; letras romanas representam coordenadas do espaço transformado $(\xi, \eta)$ e letras com apóstrofe (') representam coordenadas do espaço cartesiano $(x, y)$. Assim, os vetores de variáveis primitivas (V) e variáveis conservadas (Q), por exemplo, podem ser representados como:

$$
\mathbf{V}=V_{\alpha}=\left\{\begin{array}{c}
V_{1} \\
V_{2} \\
V_{3} \\
V_{4}
\end{array}\right\}=\left\{\begin{array}{l}
\rho \\
u \\
v \\
p
\end{array}\right\} \quad, \quad \mathbf{Q}=Q_{\beta}=\left\{\begin{array}{c}
Q_{1} \\
Q_{2} \\
Q_{3} \\
Q_{4}
\end{array}\right\}=\left\{\begin{array}{c}
\rho \\
\rho u \\
\rho v \\
e
\end{array}\right\}
$$

E a primeira variação da medida de mérito é dada por:

$$
\delta I=\int_{B_{w}} \frac{\partial g}{\partial \mathbf{Q}} \delta \mathbf{Q} \frac{\mathrm{d} s}{\mathrm{~d} \xi} \mathrm{d} \xi+\int_{B_{w}} g \delta\left(\frac{\mathrm{d} s}{\mathrm{~d} \xi}\right) \mathrm{d} \xi
$$

Note que, com a utilização da álgebra tensorial generalizada, os sistemas de coordenadas podem assumir diversas configurações. Entretanto, as propriedades do escoamento devem ser independentes do sistema de coordenadas adotado. Certamente a utilização de malhas não-estruturadas remete a um sistema de coordenadas cartesiano. Contudo, a derivação das equações adjuntas e, principalmente, a redução do seu gradiente de sensibilidade tem sua complexidade bastante reduzida ao utilizar um espaço transformado apropriado. Desta forma propõe-se desenvolver as equações do método no sistema generalizado proposto no apêndice A. Para isso, considere as equações de Euler escritas em coordenadas generalizadas, apresentadas abaixo em uma situação de estado estacionário:

$$
\frac{\partial \overline{\mathbf{E}}}{\partial \xi}+\frac{\partial \overline{\mathbf{F}}}{\partial \eta}=0
$$

Como também apresentado no apêndice A os vetores de fluxo generalizados podem ser escritos em função dos vetores de fluxo cartesianos através das seguintes 
expressões:

$$
\begin{aligned}
& \overline{\mathbf{E}}=\mathbf{E}\left(J \frac{\partial \xi}{\partial x}\right)+\mathbf{F}\left(J \frac{\partial \xi}{\partial y}\right) \\
& \overline{\mathbf{F}}=\mathbf{E}\left(J \frac{\partial \eta}{\partial x}\right)+\mathbf{F}\left(J \frac{\partial \eta}{\partial y}\right)
\end{aligned}
$$

Assim, suas respectivas variações podem ser escritas como:

$$
\begin{aligned}
\delta \overline{\mathbf{E}} & =\delta \mathbf{E}\left(J \frac{\partial \xi}{\partial x}\right)+\mathbf{E} \delta\left(J \frac{\partial \xi}{\partial x}\right)+\delta \mathbf{F}\left(J \frac{\partial \xi}{\partial y}\right)+\mathbf{F} \delta\left(J \frac{\partial \xi}{\partial y}\right) \\
\delta \overline{\mathbf{F}} & =\delta \mathbf{E}\left(J \frac{\partial \eta}{\partial x}\right)+\mathbf{E} \delta\left(J \frac{\partial \eta}{\partial x}\right)+\delta \mathbf{F}\left(J \frac{\partial \eta}{\partial y}\right)+\mathbf{F} \delta\left(J \frac{\partial \eta}{\partial y}\right)
\end{aligned}
$$

Mas, como as variações dos vetores de fluxo cartesianos podem ser escritos como mostram as equaçôes abaixo:

$$
\delta \mathbf{E}=\frac{\partial \mathbf{E}}{\partial \mathbf{Q}} \delta \mathbf{Q} \quad \text { e } \quad \delta \mathbf{F}=\frac{\partial \mathbf{F}}{\partial \mathbf{Q}} \delta \mathbf{Q}
$$

Então, ao substituir as expressões (3.8) em (3.7) e agrupar termos de modo conveniente, podem-se reescrever as variações dos vetores de fluxo generalizados da seguinte maneira:

$$
\begin{aligned}
& \delta \overline{\mathbf{E}}=\underbrace{\left[\frac{\partial \mathbf{E}}{\partial \mathbf{Q}}\left(J \frac{\partial \xi}{\partial x}\right)+\frac{\partial \mathbf{F}}{\partial \mathbf{Q}}\left(J \frac{\partial \xi}{\partial y}\right)\right]}_{\mathbf{C}_{1}} \delta \mathbf{Q}+\mathbf{E} \delta\left(J \frac{\partial \xi}{\partial x}\right) \mathbf{F} \delta\left(J \frac{\partial \xi}{\partial y}\right) \\
& \delta \overline{\mathbf{F}}=\underbrace{\left[\frac{\partial \mathbf{E}}{\partial \mathbf{Q}}\left(J \frac{\partial \eta}{\partial x}\right)+\frac{\partial \mathbf{F}}{\partial \mathbf{Q}}\left(J \frac{\partial \eta}{\partial y}\right)\right]}_{\mathbf{C}_{2}} \delta \mathbf{Q}+\mathbf{E} \delta\left(J \frac{\partial \eta}{\partial x}\right) \mathbf{F} \delta\left(J \frac{\partial \eta}{\partial y}\right)
\end{aligned}
$$

Ou ainda, de forma geral:

$$
\delta \overline{\mathcal{F}}_{\alpha}^{k}=C_{\alpha \beta}^{k} \delta Q_{\beta}+\delta\left(J \frac{\partial \xi^{k}}{\partial x^{j^{\prime}}}\right) \mathcal{F}_{\alpha}^{j^{\prime}}
$$

onde:

$$
\mathcal{F}_{\alpha}^{1}=\mathbf{E} \quad, \quad \mathcal{F}_{\alpha}^{2}=\mathbf{F} \quad, \quad \overline{\mathcal{F}}_{\alpha}^{1}=\overline{\mathbf{E}} \quad, \quad \overline{\mathcal{F}}_{\alpha}^{2}=\overline{\mathbf{F}} \quad, \quad C_{\alpha \beta}^{1}=\mathbf{C}_{1} \quad, \quad C_{\alpha \beta}^{2}=\mathbf{C}_{2}
$$

Como já visto na seção 1.2, a principal idéia do método Adjunto é impor as equações de Euler como restriçôes ao processo de otimização via a utilização de multiplicadores de Lagrange. Assim, em notação indicial, o funcional restritivo é dado por:

$$
I_{C} \equiv \int_{\mathcal{D}} \psi_{\alpha} \frac{\partial \overline{\mathcal{F}}_{\alpha}^{k}}{\partial \xi^{k}} \mathrm{~d} \mathcal{V}=0
$$


onde, o vetor de variáveis adjuntas $\Psi$ definido como:

$$
\boldsymbol{\Psi}=\psi_{\boldsymbol{\alpha}}=\left\{\begin{array}{llll}
\psi_{1} & \psi_{2} & \psi_{3} & \psi_{4}
\end{array}\right\}^{T}
$$

A variação da restrição $I_{C}$ indicada pela equação (3.11) pode ser escrita da seguinte forma:

$$
\delta I_{C}=\int_{\mathcal{D}} \psi_{\alpha} \frac{\partial\left(\delta \overline{\mathcal{F}}_{\alpha}^{k}\right)}{\partial \xi^{k}} \mathrm{~d} \mathcal{V}=\int_{\mathcal{D}} \frac{1}{J}\left\{J \psi_{\alpha} \frac{\partial\left(\delta \overline{\mathcal{F}}_{\alpha}^{k}\right)}{\partial \xi^{k}}\right\} \mathrm{d} \mathcal{V}
$$

Mas, pela regra da cadeia:

$$
\frac{\partial}{\partial \xi^{k}}\left(J \psi_{\alpha} \delta \overline{\mathcal{F}}_{\alpha}^{k}\right)=J \psi_{\alpha} \frac{\partial\left(\delta \overline{\mathcal{F}}_{\alpha}^{k}\right)}{\partial \xi^{k}}+\delta \overline{\mathcal{F}}_{\alpha}^{k} \frac{\partial\left(J \psi_{\alpha}\right)}{\partial \xi^{k}}
$$

Então, substituindo (3.14) em (3.13), obtém-se:

$$
\begin{aligned}
\delta I_{C} & =\int_{\mathcal{D}} \frac{1}{J}\left\{\frac{\partial}{\partial \xi^{k}}\left(J \psi_{\alpha} \delta \overline{\mathcal{F}}_{\alpha}^{k}\right)-\delta \overline{\mathcal{F}}_{\alpha}^{k} \frac{\partial\left(J \psi_{\alpha}\right)}{\partial \xi^{k}}\right\} \mathrm{d} \mathcal{V} \\
& =\int_{\mathcal{D}} \frac{1}{J} \frac{\partial}{\partial \xi^{k}}\left(J \psi_{\alpha} \delta \overline{\mathcal{F}}_{\alpha}^{k}\right) \mathrm{d} \mathcal{V}-\int_{\mathcal{D}} \frac{\delta \overline{\mathcal{F}}_{\alpha}^{k}}{J} \frac{\partial\left(J \psi_{\alpha}\right)}{\partial \xi^{k}} \mathrm{~d} \mathcal{V} \\
& =\underbrace{\int_{\mathcal{D}} \nabla \cdot\left(\psi_{\alpha} \delta \overline{\mathcal{F}}_{\alpha}\right) \mathrm{d} \mathcal{V}}_{1^{o} \text { termo }}-\underbrace{\int_{\mathcal{D}} \delta \overline{\mathcal{F}}_{\alpha} \nabla \cdot \psi_{\alpha} \mathrm{d} \mathcal{V}}_{2^{o} \text { termo }}
\end{aligned}
$$

Observa-se que o primeiro termo da equação (3.15) aparece, agora, na forma de um operador divergente. É possível, então, aplicar o Teorema de Gauss à este termo, transformando a integral no domínio em uma integral apenas nas suas fronteiras. Assim, a variação $\delta I_{C}$ pode ser reescrita da seguinte forma:

$$
\delta I_{C}=\int_{\partial \mathcal{D}} \psi_{\alpha} \delta \overline{\mathcal{F}}_{\alpha}^{k} n_{k} \mathrm{~d} \mathcal{S}-\int_{\mathcal{D}} \delta \overline{\mathcal{F}}_{\alpha}^{k} \frac{1}{J} \frac{\partial\left(J \psi_{\alpha}\right)}{\partial \xi^{k}} \mathrm{~d} \mathcal{V}
$$

onde $n_{k}$ o versor normal à fronteira $\partial \mathcal{D}$ apontando para fora domínio $\mathcal{D}$.

A imposição das equações de Euler como restrição ao problema de otimização leva a definição da função objetivo aumentada $I_{A}=I+I_{C}$. E sua variação é dada pela expressão abaixo:

$$
\delta I_{A}=\delta I+\delta I_{C}
$$

Note que isto não muda a função de mérito à qual se quer otimizar, já que, considerando que a solução do escoamento está convergida, o funcional restritivo que está sendo acrescentado vale zero como mostra a equação (3.5), que representa as equações de Euler (um dos fatores de $I_{C}$ ). 
Substituindo as variações (3.4) e (3.16) em (3.17), obtém-se:

$$
\begin{aligned}
\delta I_{A}= & \int_{B_{w}} \frac{\partial g}{\partial Q} \delta Q \frac{\mathrm{d} S}{\mathrm{~d} \xi} \mathrm{d} \xi+\int_{B_{w}} g \delta\left(\frac{\mathrm{d} S}{\mathrm{~d} \xi}\right) \mathrm{d} \xi-\int_{\mathcal{D}} \delta \overline{\mathcal{F}}_{\alpha}^{k} \frac{1}{J} \frac{\partial\left(J \psi_{\alpha}\right)}{\partial \xi^{k}} \mathrm{~d} \xi+ \\
& -\int_{B_{w}} \psi_{\alpha} \delta \overline{\mathcal{F}}_{\alpha}^{2} \mathrm{~d} \xi+\int_{B_{\infty}} \psi_{\alpha} \delta \overline{\mathcal{F}}_{\alpha}^{2} \mathrm{~d} \xi+ \\
& -\underbrace{\left.\int_{0}^{1} \psi_{\alpha} \delta \overline{\mathcal{F}}_{\alpha}^{1} \mathrm{~d} \eta\right|_{\xi=0}+\left.\int_{0}^{1} \psi_{\alpha} \delta \overline{\mathcal{F}}_{\alpha}^{1} \mathrm{~d} \eta\right|_{\xi=1}}_{=0\left(\left.\delta \overline{\mathcal{F}}_{\alpha}^{1}\right|_{\xi=0}=\left.\delta \overline{\mathcal{F}}_{\alpha}^{1}\right|_{\xi=1}\right)}
\end{aligned}
$$

onde $B_{w}$ representa a fronteira de parede e $B_{\infty}$ a fronteira de farfield do domínio computacional $\mathcal{D}$.

A anulação dos dois últimos termos é decorrente da condição de contorno periódica na linha de corte da malha do sistema de coordenadas generalizado. Observa-se, ainda, que na condição de contorno de parede, onde a componente contravariante do vetor velocidade $V$ do escoamento é nula, o vetor de fluxos normal à parede e sua respectiva variação ficam reduzidos à:

$$
\left.\overline{\mathcal{F}}_{\alpha}^{2}\right|_{\left(B_{w}\right)}=\left.J^{-1}\left\{\begin{array}{c}
0 \\
p \eta_{x} \\
p \eta_{y} \\
0
\end{array}\right\} \Rightarrow \delta \overline{\mathcal{F}}_{\alpha}^{2}\right|_{\left(B_{w}\right)}=J\left\{\begin{array}{c}
0 \\
\frac{\partial \eta}{\partial x} \delta p \\
\frac{\partial \eta}{\partial y} \delta p \\
0
\end{array}\right\}+p\left\{\begin{array}{c}
0 \\
\delta\left(J \frac{\partial \eta}{\partial x}\right) \\
\delta\left(J \frac{\partial \eta}{\partial y}\right) \\
0
\end{array}\right\}
$$

E substituindo as equações (3.10) e (3.19) em (3.18), chega-se à seguinte forma de $\delta I_{A}$ :

$$
\begin{aligned}
\delta I_{A}= & \int_{B_{w}} \frac{\partial g}{\partial Q_{\beta}} \delta Q_{\beta} \frac{\mathrm{d} s}{\mathrm{~d} \xi} \mathrm{d} \xi+\int_{B_{w}} g \delta\left(\frac{\mathrm{d} s}{\mathrm{~d} \xi}\right) \mathrm{d} \xi+ \\
& -\int_{\mathcal{D}} C_{\alpha \beta}^{k} \frac{1}{J} \frac{\partial\left(J \psi_{\alpha}\right)}{\partial \xi^{k}} \delta Q_{\beta} \mathrm{d} \mathcal{V}-\int_{\mathcal{D}} \delta\left(J \frac{\partial \xi^{k}}{\partial x^{j^{\prime}}}\right) \frac{\mathcal{F}_{\alpha}^{j^{\prime}}}{J} \frac{\partial\left(J \psi_{\alpha}\right)}{\partial \xi^{k}} \mathrm{~d} \mathcal{V}+ \\
& -\int_{B_{w}}\left[\psi_{2}\left(J \frac{\partial \eta}{\partial x}\right)+\psi_{3}\left(J \frac{\partial \eta}{\partial y}\right)\right] \frac{\partial p}{\partial Q_{\beta}} \delta Q_{\beta} \mathrm{d} \mathcal{V}+ \\
& -\int_{B_{w}}\left[\psi_{2} \delta\left(J \frac{\partial \eta}{\partial x}\right)+\psi_{3} \delta\left(J \frac{\partial \eta}{\partial y}\right)\right] p \mathrm{~d} \xi+\int_{B_{\infty}} \psi_{\alpha} C_{\alpha \beta}^{(2)} \delta Q_{\beta} \mathrm{d} \xi+ \\
& +\int_{B_{\infty}} \psi_{\alpha} \delta\left(J \frac{\partial \eta}{\partial x^{j^{\prime}}}\right) \mathcal{F}_{\alpha}^{j^{\prime}} \mathrm{d} \xi
\end{aligned}
$$

Rearranjando os termos de modo apropriado, é possível separar os termos dependentes de variações do escoamento $\left(\delta Q_{\beta}\right)$, que definem as equações adjuntas e suas respectivas condições de contorno, dos restantes, que fornecem o gradiente de sensibilidade em relação aos parâmetros que definem a geometria do aerofólio 
como já apresentado na seção 1.2. Desta forma, obtém-se:

$$
\begin{aligned}
\delta I_{A}= & \int_{B_{w}} \underbrace{\left\{\frac{\partial g}{\partial Q_{\beta}} \frac{\mathrm{d} s}{\mathrm{~d} \xi}-\left[\psi_{2}\left(J \frac{\partial \eta}{\partial x}\right)+\psi_{3}\left(J \frac{\partial \eta}{\partial y}\right)\right] \frac{\partial p}{\partial Q_{\beta}}\right\}}_{\text {parede }} \delta Q_{\beta} \mathrm{d} \xi+ \\
& +\int_{B_{\infty}} \underbrace{\psi_{\alpha} C_{\alpha \beta}^{(2)} \delta Q_{\beta}}_{\text {farfield }} \mathrm{d} \xi-\int_{\mathcal{D}} \underbrace{C_{\alpha \beta}^{k} \frac{\partial\left(J \psi_{\alpha}\right)}{\partial \xi^{k}}}_{\text {equação adjunta }} \delta Q_{\beta} \mathrm{d} \xi \mathrm{d} \eta+ \\
& +\underbrace{\int_{B_{w}}\left\{g \delta\left(\frac{\mathrm{d} s}{\mathrm{~d} \xi}\right)-\left[\psi_{2} \delta\left(J \frac{\partial \eta}{\partial x}\right)+\psi_{3} \delta\left(J \frac{\partial \eta}{\partial y}\right)\right] p\right\} \mathrm{d} \xi}_{1_{w}^{o} \text { termo do gradiente }}+ \\
& +\underbrace{\int_{B_{\infty}} \psi_{\alpha} \delta\left(J \frac{\partial \eta}{\partial x^{j^{\prime}}}\right) \mathcal{F}_{\alpha}^{j^{\prime}} \mathrm{d} \xi}_{2^{o} \text { termo do gradiente }}-\underbrace{\int_{\mathcal{D}} \delta\left(J \frac{\partial \xi^{k}}{\partial x^{j^{\prime}}}\right) \mathcal{F}_{\alpha}^{j^{\prime}} \frac{\left(J \psi_{\alpha}\right)}{\partial \xi^{k}} \mathrm{~d} \xi \mathrm{d} \eta}_{3^{o} \text { termo do gradiente }}
\end{aligned}
$$

Além de serem consistentes com a PDE adjunta, as condições de contorno de farfield devem eleminar o segundo termo do lado direito da equação (3.21), eliminando a dependência da variação do vetor de estado $\left(\delta Q_{\beta}\right)$ da variação $\delta I_{A}$.

\subsubsection{Redução do Gradiente}

Jameson e Kim (2003a) propuseram uma redução da expressão do gradiente para otimização de aerofólios. Para a sua derivação, considere a equação (3.21). Repare que, se a fronteira de farfield estiver suficientemente distante da superfície sólida, a variação $\delta\left(J \frac{\partial \eta}{\partial x^{j^{j}}}\right)$ do segundo termo do gradiente, é praticamente nula nesta fronteira, uma vez que as mudanças mais significativas da malha ocorrem nas proximidades do aerofólio. Além disso, $\frac{\mathrm{d} s}{\mathrm{~d} \xi}=1 \mathrm{e}$, portanto, $\delta\left(\frac{\mathrm{d} s}{\mathrm{~d} \xi}\right)=0$.

Assim, a expressão do gradiente pode ser reduzida à:

$$
\begin{aligned}
\delta I_{A}= & \int_{B_{w}}\left\{\frac{\partial g}{\partial Q_{\beta}} \frac{\mathrm{d} s}{\mathrm{~d} \xi}-\left[\psi_{2}\left(J \frac{\partial \eta}{\partial x}\right)+\psi_{3}\left(J \frac{\partial \eta}{\partial y}\right)\right] \frac{\partial p}{\partial Q_{\beta}}\right\} \delta Q_{\beta} \mathrm{d} \xi+ \\
& +\int_{B_{\infty}} \psi_{\alpha} C_{\alpha \beta}^{(2)} \delta Q_{\beta} \mathrm{d} \xi-\int_{\mathcal{D}} C_{\alpha \beta}^{k} \frac{\partial\left(J \psi_{\alpha}\right)}{\partial \xi^{k}} \delta Q_{\beta} \mathrm{d} \xi \mathrm{d} \eta+ \\
& -\int_{B_{w}}\left\{\left[\psi_{2} \delta\left(J \frac{\partial \eta}{\partial x}\right)+\psi_{3} \delta\left(J \frac{\partial \eta}{\partial y}\right)\right] p\right\} \mathrm{d} \xi+ \\
& -\int_{\mathcal{D}} \delta\left(J \frac{\partial \xi^{k}}{\partial x^{j^{\prime}}}\right) \mathcal{F}_{\alpha}^{j^{\prime}} \frac{\left(J \psi_{\alpha}\right)}{\partial \xi^{k}} \mathrm{~d} \xi \mathrm{d} \eta
\end{aligned}
$$

Definindo a matriz de transformação entre os sistemas de coordenadas cartesiano e generalizado como:

$$
S_{j^{\prime}}^{i}=J \frac{\partial \xi^{i}}{\partial x^{j^{\prime}}}
$$


É possível obter a seguinte identidade ${ }^{1}$ :

$$
\delta S_{j^{\prime}}^{i} \frac{\partial \mathcal{F}_{\alpha}^{j^{\prime}}}{\partial \xi^{i}}=-\frac{\partial}{\partial \xi^{r}}\left(S_{j^{\prime}}^{r} \frac{\partial \mathcal{F}_{\alpha}^{j^{\prime}}}{\partial \xi^{i}} \delta \xi^{i}\right)
$$

Mas,

$$
\begin{aligned}
S_{j^{\prime}}^{i} & =\frac{1}{2} \epsilon_{j^{\prime} p^{\prime} q^{\prime}} \epsilon^{i r s} \frac{\partial x^{p^{\prime}}}{\partial \xi^{r}} \frac{\partial x^{q^{\prime}}}{\partial \xi^{s}} \\
\delta S_{j^{\prime}}^{i} & =\frac{1}{2} \epsilon_{j^{\prime} p^{\prime} q^{\prime}} \epsilon^{i r s}\left[\frac{\partial\left(\delta x^{p^{\prime}}\right)}{\partial \xi^{r}} \frac{\partial x^{q^{\prime}}}{\partial \xi^{s}}+\frac{\partial x^{p^{\prime}}}{\partial \xi^{r}} \frac{\partial\left(\delta x^{q^{\prime}}\right)}{\partial \xi^{s}}\right] \\
\frac{\partial\left(\delta S_{j^{\prime}}^{i}\right)}{\partial \xi^{i}} & =\frac{1}{2} \epsilon_{j^{\prime} p^{\prime} q^{\prime}} \epsilon^{i r s} \frac{\partial}{\partial \xi^{i}}\left[\frac{\partial\left(\delta x^{p^{\prime}}\right)}{\partial \xi^{r}} \frac{\partial x^{q^{\prime}}}{\partial \xi^{s}}+\frac{\partial x^{p^{\prime}}}{\partial \xi^{r}} \frac{\partial\left(\delta x^{q^{\prime}}\right)}{\partial \xi^{s}}\right]=0
\end{aligned}
$$

E pela regra da cadeia:

$$
\frac{\partial}{\partial \xi^{i}}\left(\delta S_{j^{\prime}}^{i} \mathcal{F}_{\alpha}^{j^{\prime}}\right)=\frac{\partial\left(\delta S_{j^{\prime}}^{i}\right)^{\sigma^{0}}}{\partial \xi^{i}} \mathcal{F}_{\alpha}^{j^{\prime}}+\delta S_{j^{\prime}}^{i} \frac{\partial \mathcal{F}_{\alpha}^{j^{\prime}}}{\partial \xi^{i}}
$$

Note que o termo anulado se refere a variações de geometria, o que não ocorre durante uma solução adjunta. Então:

$$
\begin{aligned}
\frac{\partial}{\partial \xi^{i}}\left(\delta S_{j^{\prime}}^{i} \mathcal{F}_{\alpha}^{j^{\prime}}\right) & =-\frac{\partial}{\partial \xi^{r}}\left(S_{j^{\prime}}^{r} \frac{\partial \mathcal{F}_{\alpha}^{j^{\prime}}}{\partial Q_{\beta}} \frac{\partial Q_{\beta}}{\partial \xi^{k}} \delta \xi^{k}\right) \\
& =-\frac{\partial}{\partial \xi^{r}}\left(S_{j^{\prime}}^{r} A_{\alpha \beta}^{j^{\prime}} \frac{\partial Q_{\beta}}{\partial \xi^{k}} \delta \xi^{k}\right) \\
& =-\frac{\partial}{\partial \xi^{r}}\left(C_{\alpha \beta}^{r} \delta Q_{\beta}^{*}\right)
\end{aligned}
$$

O último termo da equação (3.22) pode ser reescrito na forma:

$$
\begin{aligned}
& \int_{\mathcal{D}} \delta S_{j^{\prime}}^{i} \mathcal{F}_{\alpha}^{j^{\prime}} \frac{\partial\left(J \psi_{\alpha}\right)}{\partial \xi^{i}} \mathrm{~d} \xi \mathrm{d} \eta=\int_{\mathcal{D}} \delta S_{j^{\prime}}^{i} \mathcal{F}_{\alpha}^{j^{\prime}} \frac{1}{J} \frac{\partial\left(J \psi_{\alpha}\right)}{\partial \xi^{i}} \mathrm{~d} A= \\
= & \int_{\mathcal{D}} \frac{1}{J} \frac{\partial}{\partial \xi^{i}}\left[J\left(\psi_{\alpha} \delta S_{j^{\prime}}^{i} \mathcal{F}_{\alpha}^{j^{\prime}}\right)\right] \mathrm{d} A-\int_{\mathcal{D}} \frac{J}{J} \psi_{\alpha} \frac{\partial}{\partial \xi^{i}}\left(\delta S_{j^{\prime}}^{i} \mathcal{F}_{\alpha}^{j^{\prime}}\right) \mathrm{d} A= \\
= & \int_{\partial \mathcal{D} \equiv B_{\infty}+B_{w}} \psi_{\alpha} \delta S_{j^{\prime}}^{i} \mathcal{F}_{\alpha}^{j^{\prime}} n_{i} \mathrm{~d} \xi+\int_{\mathcal{D}} \psi_{\alpha} \frac{\partial}{\partial \xi^{i}}\left(C_{\alpha \beta}^{i} \delta Q_{\beta}^{*}\right) \mathrm{d} A= \\
= & \int_{\partial \mathcal{D}} \psi_{\alpha} \delta S_{j^{\prime}}^{i} \mathcal{F}_{\alpha}^{j^{\prime}} n_{i} \mathrm{~d} \xi+\int_{\mathcal{D}} \frac{J}{J} \psi_{\alpha} \frac{\partial}{\partial \xi^{i}}\left(C_{\alpha \beta}^{i} \delta Q_{\beta}^{*}\right) \mathrm{d} A= \\
= & \int_{\partial \mathcal{D}} \psi_{\alpha} \delta S_{j^{\prime}}^{i} \mathcal{F}_{\alpha}^{j^{\prime}} n_{i} \mathrm{~d} \xi+\int_{\mathcal{D}} \frac{1}{J} \frac{\partial}{\partial \xi^{i}}\left(J \psi_{\alpha} C_{\alpha \beta}^{i} \delta Q_{\beta}^{*}\right) \mathrm{d} A-\int_{\mathcal{D}} C_{\alpha \beta}^{i} \delta Q_{\beta}^{*} \frac{1}{J} \frac{\partial\left(J \psi_{\alpha}\right)}{\partial \xi^{i}} \mathrm{~d} A= \\
= & \int_{\partial \mathcal{D}} \psi_{\alpha}\left(\delta S_{j^{\prime}}^{i} \mathcal{F}_{\alpha}^{j^{\prime}}+C_{\alpha \beta}^{i} \delta Q_{\beta}^{*}\right) n_{i} \mathrm{~d} \xi-\int_{\mathcal{D}} C_{\alpha \beta}^{i} \delta Q_{\beta}^{*} \frac{1}{J} \frac{\partial\left(J \psi_{\alpha}\right)}{\partial \xi^{i}} \mathrm{~d} A
\end{aligned}
$$

\footnotetext{
${ }^{1}$ a demonstração encontra-se no apêndice $B$
} 
Ou, ainda, separando as integrais nas fronteiras:

$$
\begin{aligned}
\int_{\mathcal{D}} \delta S_{j^{\prime}}^{i} \mathcal{F}_{\alpha}^{j^{\prime}} \frac{\partial\left(J \psi_{\alpha}\right)}{\partial \xi^{i}} \mathrm{~d} \xi \mathrm{d} \eta= & \int_{B_{\infty}} \psi_{\alpha}\left(\delta S_{j^{\prime}}^{i} \mathcal{F}_{\alpha}^{j^{\prime}}+C_{\alpha \beta}^{i} \delta Q_{\beta}^{*}\right) n_{i} \mathrm{~d} \xi+ \\
& -\int_{\mathcal{D}} C_{\alpha \beta}^{i} \frac{1}{J} \frac{\partial\left(J \psi_{\alpha}\right)}{\partial \xi^{i}} \delta Q_{\beta}^{*} \mathrm{~d} A+ \\
& +\int_{B_{w}} \psi_{\alpha}\left(\delta S_{j^{\prime}}^{2} \mathcal{F}_{\alpha}^{j^{\prime}}+C_{\alpha \beta}^{2} \delta Q_{\beta}^{*}\right) \mathrm{d} \xi
\end{aligned}
$$

onde,

$$
\mathrm{d} A=J \mathrm{~d} \xi \mathrm{d} \eta \quad ; \quad \frac{1}{J} \frac{\partial\left(J \psi_{\alpha}\right)}{\partial \xi^{i}}=\frac{\nabla\left(J \psi_{\alpha}\right)}{J}
$$

Assim, rearranjando os termos da equação de forma apropriada, tem-se:

$$
\begin{aligned}
\delta I_{A}= & \int_{B_{w}} \underbrace{\left\{\frac{\partial g}{\partial Q_{\beta}}-\left[\psi_{2}\left(J \frac{\partial \eta}{\partial x}\right)+\psi_{3}\left(J \frac{\partial \eta}{\partial y}\right)\right] \frac{\partial p}{\partial Q_{\beta}}\right\}}_{\text {parede }} \delta Q_{\beta} \mathrm{d} \xi+ \\
& +\int_{B_{\infty}} \underbrace{\psi_{\alpha} C_{\alpha \beta}^{(2)}\left(\delta Q_{\beta}-\delta Q_{\beta}^{*}\right)}_{\text {farfield }} \mathrm{d} \xi-\int_{\mathcal{D}} \underbrace{C_{\alpha \beta}^{i} \frac{\partial}{\partial \xi^{i}}\left(J \psi_{\alpha}\right)}_{\text {eq. adjunta }}\left(\delta Q_{\beta}-\delta Q_{\beta}^{*}\right) \mathrm{d} \xi \mathrm{d} \eta+ \\
& +\underbrace{\int_{B^{\circ} \text { termo do gradiente }}}_{1_{w}^{o} \psi_{\alpha}\left(\delta S_{j^{\prime}}^{2} \mathcal{F}_{\alpha}^{j^{\prime}}+C_{\alpha \beta}^{(2)} \delta Q_{\beta}^{*}\right) \mathrm{d} \xi}+\underbrace{\int_{p \mathrm{~d} \xi}}_{\psi_{B_{w}}\left[\psi_{2} \delta\left(J \frac{\partial \eta}{\partial x}\right)+\psi_{3} \delta\left(J \frac{\partial \eta}{\partial y}\right)\right]}
\end{aligned}
$$

Considerando a transformação de coordenadas localmente em cada volume da malha, pode se encará- la como uma simples rotação do sistema de coordenadas. Assim, $J=1$ e então, as equações adjuntas em coordenadas cartesianas podem ser escritas na forma:

$$
\mathbf{A}^{T} \frac{\partial \Psi}{\partial x}+\mathbf{B}^{T} \frac{\partial \Psi}{\partial y}=0
$$

Sujeitas a condição de contorno de parede sólida dada por:

$$
\left[\psi_{2}\left(J \frac{\partial \eta}{\partial x}\right)+\psi_{3}\left(J \frac{\partial \eta}{\partial y}\right)\right] \frac{\partial p}{\partial Q_{\beta}}=\frac{\partial g}{\partial Q_{\beta}}
$$

E pela condição de contorno de farfield expressa por:

$$
\psi_{\alpha} C_{\alpha \beta}^{(2)}\left(\delta Q_{\beta}-\delta Q_{\beta}^{*}\right)=0 \quad, \quad C_{\alpha \beta}^{(2)}=\mathbf{A}_{n}^{T}=k_{x} \mathbf{A}^{T}+k_{y} \mathbf{B}^{T}
$$

Note que, com essa redução é possível determinar o gradiente de sensibilidade apenas com integrais no contorno do aerofólio, diminuindo sensivelmente o 
custo computacional desta etapa do ciclo de projeto. Observe, no entanto, que a implementação da otimização de turbo máquinas (ou qualquer outra superfície aerodinâmica que esteja sujeita à escoamentos internos, onde as fronteiras de entrada e saída estão próximas a geometria que se quer otimizar), por exemplo, requer a utilização da forma completa do gradiente dada pela equação (3.21).

\subsection{Método Numérico}

Devido à semelhança das equações adjuntas às equações que governam o escoamento, é natural utilizar os mesmos métodos para sua resolução. Observe que, para resolver as equações adjuntas em estado estacionário, é conveniente incluir um termo tempo dependente como feito nas equações de Euler. Assim, obtém- se a seguinte equação:

$$
\frac{\partial \Psi}{\partial t}-\mathbf{A}^{T} \frac{\partial \Psi}{\partial x}-\mathbf{B}^{T} \frac{\partial \Psi}{\partial y}=0
$$

Veja que o sinal negativo dos termos convectivos não altera o resultado estacionário das equações adjuntas. No entanto, a idéia de utilizá-lo durante a solução "transiente" está relacionada com a direção de propagação da informação (características), que permitirá o tratamento das condições de contorno proposto no capítulo 4.

Entretanto, três modificações foram feitas ao esquema numérico de modo a adaptá- lo para tratar as equações adjuntas. Primeiro, uma vez que as equações adjuntas aparecem em forma quase-linear não-conservativa, os termos convectivos devem ser calculados de uma maneira diferente. As derivadas $\frac{\partial \psi}{\partial x_{i}}$ são calculadas da mesma forma que as derivadas espaciais das propriedades do escoamento (seção 2.3.3) e em seguida este vetor é multiplicado pelas respectivas matrizes jacobianas. Segundo, a direção da integração do tempo até o estado estacionário é reversa em relação à integração da solução do escoamento, já que as direções de propagação de ondas são reversas. Terceiro, como os termos de dissipação artificial são calculados pelas mesmas subrotinas que a solução do escoamento, eles são adicionados ao invés de subtraídos ao termo convectivo (JAMESON; SRIRAM; MARTINELLI, 2003), que é calculado pela expressão:

$$
C\left(\boldsymbol{\Psi}_{i}\right)=\mathbf{A}_{i}^{T}\left[\frac{1}{\mathcal{V}_{i}} \sum_{k} \boldsymbol{\Psi}_{i k} \Delta y_{i k}\right]+\mathbf{B}_{i}^{T}\left[-\frac{1}{\mathcal{V}_{i}} \sum_{k} \boldsymbol{\Psi}_{i k} \Delta x_{i k}\right]
$$

onde,

$$
\Psi_{i k}=\frac{1}{2}\left(\boldsymbol{\Psi}_{i}+\boldsymbol{\Psi}_{k}\right)
$$


Vale lembrar que a solução é realizada em uma malha não-estruturada de triângulos, como mostra a figura 2.1(a).

\subsubsection{Dissipação Artificial}

Note que as equações adjuntas, ao contrário das equações de Euler, são lineares, o que implica no não aparecimento de instabilidades devido ao cascateamento de freqüências. Entretanto, ao discretizá-las de maneira centrada como mostra a equação (3.41), existe a possibilidade do aparecimento oscilações na solução do tipo odd-even decoupling como mostra Hirsch (1988a). Desta forma, com a utilização de um esquema centrado, faz-se necessária a implementação de dissipação artificial, para amenizar possíveis instabilidades. Reuther (1996) propõe a utilização do mesmo operador de dissipação artificial aplicado às equações de Euler para resolver as equações adjuntas. Assim:

$$
D\left(\mathbf{\Psi}_{i}\right)=d^{(2)}\left(\boldsymbol{\Psi}_{i}\right)+d^{(4)}\left(\boldsymbol{\Psi}_{i}\right)
$$

O operador Laplaciano não-dividido, $d^{(2)}\left(\boldsymbol{\Psi}_{i}\right)$, é escrito na forma:

$$
d^{(2)}\left(\boldsymbol{\Psi}_{i}\right)=\sum_{k}\left[\frac{1}{2}\left(A_{i}+A_{k}\right) \epsilon_{i k}^{(2)}\left(\boldsymbol{\Psi}_{k}-\boldsymbol{\Psi}_{i}\right)\right]
$$

onde,

$$
\epsilon_{i k}^{(2)}=k^{(2)} \max \left(\nu_{i}, \nu_{k}\right) \quad \text { e } \quad \nu_{i}=\frac{\sum_{k}\left|p_{k}-p_{i}\right|}{\sum_{k}\left(p_{k}+p_{i}\right)}
$$

Enquanto o operador bi-harmônico, $d^{(4)}\left(\boldsymbol{\Psi}_{i}\right)$, é dado por:

$$
d^{(4)}\left(\Psi_{i}\right)=\sum_{k}\left[\frac{1}{2}\left(A_{i}+A_{k}\right) \epsilon_{i k}^{(4)}\left(\nabla^{2} \Psi_{k}-\nabla^{2} \Psi_{i}\right)\right]
$$

onde,

$$
\epsilon_{i k}^{(4)}=\max \left[0,\left(k^{(4)}-\epsilon_{i k}^{(2)}\right)\right] \quad \text { e } \quad \nabla^{2} \Psi_{i} \equiv \sum_{k}\left(\Psi_{k}-\Psi_{i}\right)
$$

O termo $\frac{1}{2}\left(A_{i}+A_{k}\right)$ é um fator de escalonamento dos termos de dissipação artificial. Neste modelo de dissipação artificial proposto, faz-se este termo proporcional à magnitude do maior autovalor (velocidade característica) das equações de Euler na direção normal à face.

$$
A_{i}=\sum_{k}\left[\left|\overrightarrow{\mathbf{v}}_{i k} \cdot \vec{S}_{i k}\right|+a_{i k}\left|\vec{S}_{i k}\right|\right]
$$


onde $\overrightarrow{\mathbf{v}}_{i k}$ é a velocidade do fluido na face que une os elementos $i$ e $k$ dada por:

$$
\overrightarrow{\mathbf{v}}_{i k}=u_{i k} \hat{i}_{x}+v_{i k} \hat{i}_{y}
$$

As constantes utilizadas são $k^{(2)}=1 / 4$ e $k^{(4)}=3 / 256$.

\subsubsection{Esquema de Marcha no Tempo}

O esquema de marcha no tempo adotado é o mesmo utilizado para a resolução do escoamento, com a única diferença no sinal dos termos convectivo e difusivo, uma vez que o sentido de propagação de informação, entenda-se características, é o contrário.

Ao se discretizar as equações adjuntas espacialmente, obtém-se a ODE abaixo:

$$
\frac{\mathrm{d} \boldsymbol{\Psi}_{i}}{\mathrm{~d} t}=\underbrace{\frac{1}{V_{i}}\left[C\left(\boldsymbol{\Psi}_{i}\right)+D\left(\boldsymbol{\Psi}_{i}\right)\right]}_{\text {Resíduo }}
$$

O Runge-Kutta de 5 passos e $2^{\mathrm{a}}$ ordem de precisão é dado por:

$$
\begin{aligned}
\boldsymbol{\Psi}_{i}^{(0)} & =\boldsymbol{\Psi}_{i}^{(n)} \\
\boldsymbol{\Psi}_{i}^{(1)} & =\boldsymbol{\Psi}_{i}^{(0)}+\alpha_{1} \frac{\Delta t_{i}}{V_{i}}\left[C\left(\boldsymbol{\Psi}_{i}^{(0)}\right)+D\left(\boldsymbol{\Psi}_{i}^{(0)}\right)\right] \\
\boldsymbol{\Psi}_{i}^{(2)} & =\boldsymbol{\Psi}_{i}^{(0)}+\alpha_{2} \frac{\Delta t_{i}}{V_{i}}\left[C\left(\boldsymbol{\Psi}_{i}^{(1)}\right)+D\left(\boldsymbol{\Psi}_{i}^{(1)}\right)\right] \\
\boldsymbol{\Psi}_{i}^{(3)} & =\boldsymbol{\Psi}_{i}^{(0)}+\alpha_{3} \frac{\Delta t_{i}}{V_{i}}\left[C\left(\boldsymbol{\Psi}_{i}^{(2)}\right)+D\left(\boldsymbol{\Psi}_{i}^{(1)}\right)\right] \\
\boldsymbol{\Psi}_{i}^{(4)} & =\Psi_{i}^{(0)}+\alpha_{4} \frac{\Delta t_{i}}{V_{i}}\left[C\left(\boldsymbol{\Psi}_{i}^{(3)}\right)+D\left(\boldsymbol{\Psi}_{i}^{(1)}\right)\right] \\
\boldsymbol{\Psi}_{i}^{(5)} & =\Psi_{i}^{(0)}+\alpha_{5} \frac{\Delta t_{i}}{V_{i}}\left[C\left(\boldsymbol{\Psi}_{i}^{(4)}\right)+D\left(\boldsymbol{\Psi}_{i}^{(1)}\right)\right] \\
\boldsymbol{\Psi}_{i}^{(n+1)} & =\Psi_{i}^{(5)}
\end{aligned}
$$

Onde os coeficientes utilizados são $\alpha_{1}=\frac{1}{4}, \alpha_{2}=\frac{1}{6}, \alpha_{3}=\frac{3}{8}, \alpha_{4}=\frac{1}{2}, \alpha_{5}=1$. 


\section{Condições DE CONTORNO DO Problema Adjunto}

Como foi visto no capítulo 2, a formulação característica das equações de Euler basea-se na idéia de propagação de informações do escoamento na direção normal à uma determinada fronteira. Este trabalho propõe a construção de um problema de contorno adjunto completamente análogo ao que foi feito com as equações que governam o escoamento. O problema dual é formulado de tal maneira que as suas características que se propagam no sentido contrário das do problema primal. Assim, o número de características que "saem" do domínio no problema primal é o mesmo número de características que "entram" no domínio no problema dual, e vice-versa.

\subsection{Parede}

No capítulo anterior, viu-se que as equações adjuntas devem ser resolvidas no domínio e satisfazer suas condições de contorno nas fronteiras. Na parede a condição que deve ser satisfeita é a seguinte:

$$
\left[\psi_{2}\left(J \frac{\partial \eta}{\partial x}\right)+\psi_{3}\left(J \frac{\partial \eta}{\partial y}\right)\right]=\frac{\partial g}{\partial Q_{\beta}} \frac{\partial Q_{\beta}}{\partial p}
$$

Note que, no esquema numérico utilizado, onde o valor das variáveis adjuntas na face é aproximado por um método de diferenças centradas, a condição de contorno de parede pode ser discretizada na forma:

$$
J\left[\frac{\partial \eta}{\partial x}\left(\frac{\psi_{2}^{\text {ghost }}+\psi_{2}^{\text {int }}}{2}\right)+\frac{\partial \eta}{\partial y}\left(\frac{\psi_{3}^{\text {ghost }}+\psi_{3}^{\text {int }}}{2}\right)\right]=\frac{\partial g}{\partial Q_{\beta}} \frac{\partial Q_{\beta}}{\partial p}
$$

Entretanto, existem muitas formas de definir as variáveis adjuntas nos volumes "fantasmas" de modo a respeitar a condição de contorno de parede sólida na fronteira, uma vez que há apenas uma equação e duas incógnitas. Este trabalho não tem como principal objetivo o estudo da condição de parede, mas da con- 
dição de farfield, que é pouco explorada na literatura. Desta forma, faz se uso de uma das 3 condições de contorno de parede propostas por Reuther (1996) e apresentada abaixo:

$$
\begin{aligned}
\psi_{1}^{\text {ghost }} & =\psi_{1}^{\text {int }} \\
\psi_{2}^{\text {ghost }} & =\psi_{2}^{\text {int }}-2 \Phi J \frac{\partial \eta}{\partial x}\left[\frac{\partial g}{\partial Q_{\beta}} \frac{\partial Q_{\beta}}{\partial p} \frac{\mathrm{d} s}{\mathrm{~d} \xi}+J \frac{\partial \eta}{\partial x} \psi_{2}^{\text {int }}+J \frac{\partial \eta}{\partial y} \psi_{3}^{\text {int }}\right] \\
\psi_{3}^{\text {ghost }} & =\psi_{3}^{\text {int }}-2 \Phi J \frac{\partial \eta}{\partial y}\left[\frac{\partial g}{\partial Q_{\beta}} \frac{\partial Q_{\beta}}{\partial p} \frac{\mathrm{d} s}{\mathrm{~d} \xi}+J \frac{\partial \eta}{\partial x} \psi_{2}^{\text {int }}+J \frac{\partial \eta}{\partial y} \psi_{3}^{\text {int }}\right] \\
\psi_{4}^{\text {ghost }} & =\psi_{4}^{\text {int }}
\end{aligned}
$$

onde,

$$
\Phi=\frac{1}{J^{2}\left[\left(\frac{\partial \eta}{\partial x}\right)^{2}+\left(\frac{\partial \eta}{\partial y}\right)^{2}\right]}
$$

Note que estas equações podem ser escritas, utilizando a transformação de coordenadas local, no sistema cartesiano da seguinte forma:

$$
\begin{aligned}
\psi_{1}^{\text {ghost }} & =\psi_{1}^{\text {int }} \\
\psi_{2}^{\text {ghost }} & =\psi_{2}^{\text {int }}-2 k_{x}\left[\frac{\partial g}{\partial Q_{\beta}} \frac{\partial Q_{\beta}}{\partial p}+k_{x} \psi_{2}^{\text {int }}+k_{y} \psi_{3}^{\text {int }}\right] \\
\psi_{3}^{\text {ghost }} & =\psi_{3}^{\text {int }}-2 k_{y}\left[\frac{\partial g}{\partial Q_{\beta}} \frac{\partial Q_{\beta}}{\partial p}+k_{x} \psi_{2}^{\text {int }}+k_{y} \psi_{3}^{\text {int }}\right] \\
\psi_{4}^{\text {ghost }} & =\psi_{4}^{\text {int }}
\end{aligned}
$$

Note que a extrapolação de $\psi_{1}$ e $\psi_{4}$ na parede equivale a fazer:

$$
\frac{\partial \psi_{1}}{\partial \eta}=0 \quad, \quad \frac{\partial \psi_{4}}{\partial \eta}=0
$$

Adotando uma medida de mérito de projeto inverso como apresentado pela equação (1.1), obtém-se:

$$
\begin{aligned}
\psi_{1}^{\text {ghost }} & =\psi_{1}^{\text {int }} \\
\psi_{2}^{\text {ghost }} & =\psi_{2}^{\text {int }}-2 k_{x}\left[\left(p-p_{d}\right)+k_{x} \psi_{2}^{\text {int }}+k_{y} \psi_{3}^{\text {int }}\right] \\
\psi_{3}^{\text {ghost }} & =\psi_{3}^{\text {int }}-2 k_{y}\left[\left(p-p_{d}\right)+k_{x} \psi_{2}^{\text {int }}+k_{y} \psi_{3}^{\text {int }}\right] \\
\psi_{4}^{\text {ghost }} & =\psi_{4}^{\text {int }}
\end{aligned}
$$




\subsection{Farfield}

As condições de contorno de farfield são baseadas na formulação característica das equações adjuntas, obtidas de forma completamente análoga à formulação características das equações que governam o escoamento. Assim, considere a equação adjunta:

$$
\frac{\partial \Psi}{\partial t}-\mathbf{A}^{T} \frac{\partial \Psi}{\partial x}-\mathbf{B}^{T} \frac{\partial \Psi}{\partial y}=0
$$

Apesar de linear a equação adjunta retém a hiperbolicidade das equações de Euler, já que suas matrizes jacobianas $\mathbf{A}^{T}$ e $\mathbf{B}^{T}$ são as mesmas, apenas transpostas. Assim, é possível definir uma matriz $\mathbf{A}_{n}^{T}$, tal que:

$$
\mathbf{A}_{n}^{T}=-k_{x} \mathbf{A}^{T}-k_{y} \mathbf{B}^{T}
$$

Que pode, então, ser diagonalizada da seguinte maneira:

$$
\Lambda^{T}=\mathbf{P}^{T} \mathbf{A}_{n}^{T}\left(\mathbf{P}^{T}\right)^{-1}
$$

Obtendo a matriz jacobiana da direção normal à fronteira:

$$
\boldsymbol{\Lambda}=\left[\begin{array}{cccc}
-\left(k_{x} u+k_{y} v\right) & 0 & 0 & 0 \\
0 & -\left(k_{x} u+k_{y} v\right) & 0 & 0 \\
0 & 0 & -\left(k_{x} u+k_{y} v+c\right) & 0 \\
0 & 0 & 0 & -\left(k_{x} u+k_{y} v-c\right)
\end{array}\right]
$$

O motivo dos sinais negativos das equações adjuntas fica claro quando se obtém a matriz diagonalizada $\Lambda$. Observa- se que os autovalores são os mesmos das matrizes jacobianas do escoamento, porém com sinal contrário. Desta forma, pode-se utilizar a informação do escoamento para complementar as condições de contorno do método adjunto, garantindo que o problema seja tão bem-posto quanto o problema primal.

Multiplicando a equação (4.8) por $\mathbf{P}^{T}$, obtém-se:

$$
\mathbf{P}^{T} \frac{\partial \Psi}{\partial t}-\mathbf{P}^{T} \mathbf{A}^{T} \frac{\partial \Psi}{\partial x}-\mathbf{P}^{T} \mathbf{B}^{T} \frac{\partial \Psi}{\partial y}=0
$$

Mas, como $\left(\mathbf{P}^{T}\right)^{-1} \mathbf{P}^{T}=I$, então:

$$
\mathbf{P}^{T} \frac{\partial \Psi}{\partial t}-\mathbf{P}^{T} \mathbf{A}^{T}\left(\mathbf{P}^{T}\right)^{-1} \mathbf{P}^{T} \frac{\partial \Psi}{\partial x}-\mathbf{P}^{T} \mathbf{B}^{T}\left(\mathbf{P}^{T}\right)^{-1} \mathbf{P}^{T} \frac{\partial \Psi}{\partial y}=0
$$


Expandindo a equação acima, obtém-se:

$$
\begin{aligned}
& \frac{\partial \psi_{1}}{\partial t}+u \frac{\partial \psi_{2}}{\partial t}+v \frac{\partial \psi_{3}}{\partial t}+=u \frac{\partial \psi_{1}}{\partial x}+u^{2} \frac{\partial \psi_{2}}{\partial x}+u v \frac{\partial \psi_{3}}{\partial x}+\frac{u\left(u^{2}+v^{2}\right)}{2} \frac{\partial \psi_{4}}{\partial x}+ \\
& +\frac{\left(u^{2}+v^{2}\right)}{2} \frac{\partial \psi_{4}}{\partial t}=+v \frac{\partial \psi_{1}}{\partial y}+u v \frac{\partial \psi_{2}}{\partial y}+v^{2} \frac{\partial \psi_{3}}{\partial y}+\frac{v\left(u^{2}+v^{2}\right)}{2} \frac{\partial \psi_{4}}{\partial y} \\
& \rho k_{y} \frac{\partial \psi_{1}}{\partial x}+2 \rho k_{y} u \frac{\partial \psi_{2}}{\partial x}-\rho\left(k_{x} u-k_{y} v\right) \frac{\partial \psi_{3}}{\partial x}+ \\
& \rho k_{y} \frac{\partial \psi_{2}}{\partial t}-\rho k_{x} \frac{\partial \psi_{3}}{\partial t}+=-\left[\rho k_{x} u v-\frac{\rho k_{y}\left(3 u^{2}+v^{2}\right)}{2}-\frac{\rho c^{2} k_{y}}{\gamma-1}\right] \frac{\partial \psi_{4}}{\partial x}+ \\
& +\rho\left(k_{y} u-k_{x} v\right) \frac{\partial \psi_{4}}{\partial t}=-\rho k_{x} \frac{\partial \psi_{1}}{\partial y}-\rho\left(k_{x} u-k_{y} v\right) \frac{\partial \psi_{2}}{\partial y}-2 \rho k_{x} v \frac{\partial \psi_{3}}{\partial y}+ \\
& +\left[\rho k_{y} u v-\frac{\rho k_{x}\left(u^{2}+3 v^{2}\right)}{2}-\frac{\rho c^{2} k_{x}}{\gamma-1}\right] \frac{\partial \psi_{4}}{\partial y} \\
& \rho \frac{\partial \psi_{1}}{\partial t}+\rho\left(u+k_{x} c\right) \frac{\partial \psi_{2}}{\partial t}+\quad-\left[\rho c^{2} k_{y}\left(k_{x} v-k_{y} u\right)-\frac{\rho}{2}\left(u+k_{x} c\right)\left(u^{2}+v^{2}\right)+\right. \\
& \left.+\rho\left(v+k_{y} c\right) \frac{\partial \psi_{3}}{\partial t}+\quad-\rho c\left(u+k_{x} c\right)\left(k_{x} u+k_{y} v\right)-\frac{\rho c^{2}\left(u+k_{x} c\right)}{\gamma-1}\right] \frac{\partial \psi_{4}}{\partial x}+ \\
& {\left[\frac{\rho c^{2}}{\gamma-1}+\rho c\left(k_{x} u+k_{y} v\right)+=+\rho\left(v+k_{y} c\right) \frac{\partial \psi_{1}}{\partial y}-\left[\rho c^{2} k_{x} k_{y}+\right.\right.} \\
& \left.\left.+\frac{\rho}{2}\left(u^{2}+v^{2}\right)\right] \frac{\partial \psi_{4}}{\partial t} \quad-\rho\left(u+k_{x} c\right)\left(v+k_{y} c\right)\right] \frac{\partial \psi_{2}}{\partial y}+\left[\rho c^{2} k_{x}^{2}+\right. \\
& \left.+\rho\left(v+k_{y} c\right)^{2}\right] \frac{\partial \psi_{3}}{\partial y}-\left[\rho c^{2} k_{x}\left(k_{y} u-k_{x} v\right)+\right. \\
& -\frac{\rho c^{2}\left(v+k_{y} c\right)}{\gamma-1}-\rho c\left(v+k_{y} c\right)\left(k_{x} u+k_{y} v\right)+ \\
& \left.-\frac{\rho}{2}\left(v+k_{y} c\right)\left(u^{2}+v^{2}\right)\right] \frac{\partial \psi_{4}}{\partial y} \\
& \rho \frac{\partial \psi_{1}}{\partial t}+\rho\left(u-k_{x} c\right) \frac{\partial \psi_{2}}{\partial t}+ \\
& \left.+\rho\left(v-k_{y} c\right) \frac{\partial \psi_{3}}{\partial t}+\quad-\rho c\left(u-k_{x} c\right)\left(k_{x} u+k_{y} v\right)+\frac{\rho c^{2}\left(u-k_{x} c\right)}{\gamma-1}\right] \frac{\partial \psi_{4}}{\partial x}+ \\
& {\left[\frac{\rho c^{2}}{\gamma-1}-\rho c\left(k_{x} u+k_{y} v\right)+=+\rho\left(v-k_{y} c\right) \frac{\partial \psi_{1}}{\partial y}-\left[\rho c^{2} k_{x} k_{y}+\right.\right.} \\
& \begin{array}{ll}
\left.+\frac{\rho}{2}\left(u^{2}+v^{2}\right)\right] \frac{\partial \psi_{4}}{\partial t} & \left.+\rho\left(u-k_{x} c\right)\left(v-k_{y} c\right)\right] \frac{\partial \psi_{2}}{\partial y}+\left[\rho c^{2} k_{x}^{2}+\right. \\
& \left.-\rho\left(v-k_{y} c\right)^{2}\right] \frac{\partial \psi_{3}}{\partial y}-\left[\rho c^{2} k_{x}\left(k_{y} u-k_{x} v\right)+\right.
\end{array} \\
& -\frac{\rho c^{2}\left(v-k_{y} c\right)}{\gamma-1}+\rho c\left(v-k_{y} c\right)\left(k_{x} u+k_{y} v\right)+ \\
& \left.-\quad \frac{\rho}{2}\left(v-k_{y} c\right)\left(u^{2}+v^{2}\right)\right] \frac{\partial \psi_{4}}{\partial y}
\end{aligned}
$$

Que nada mais é do que a formulação característica das equações adjuntas para o problema Euler bidimensional. Com ela, assim como na condição de contorno de farfield do escoamento, é possível determinar o vetor de variáveis adjuntas completo nos volumes "fantasmas" à partir da informação que propaga 
do domínio em direção à fronteira.

Assim como nas equações características do escoamento, observa-se que as 2 primeiras equações estão relacionadas com a velocidade normal a fronteira $-\overrightarrow{\mathbf{v}} \cdot \vec{n}$, enquanto a $3^{\mathrm{a}}$ e $4^{\mathrm{a}}$ equações estão relacionadas à ondas acústicas através das características $-(\overrightarrow{\mathbf{v}} \cdot \vec{n}+c) \mathrm{e}-(\overrightarrow{\mathbf{v}} \cdot \vec{n}-c)$, respectivamente.

Afim de simplificar a notação, este sistema pode ser escrito ao definir os coeficientes das variáveis adjuntas separadamente como constantes formadas por propriedades do escoamento. Aqui, vale lembrar que a solução adjunta é obtida à partir de uma solução estacionária do escoamento e, portanto, todas as propriedades físicas são constantes espacialmente no processo de resolução do problema dual. Desta forma, obtém-se as seguintes expressões:

$$
\left\{\begin{aligned}
C_{11 t} \frac{\partial \psi_{1}}{\partial t}+C_{12 t} \frac{\partial \psi_{2}}{\partial t}+= & \\
+C_{13 t} \frac{\partial \psi_{3}}{\partial t}+C_{14 t} \frac{\partial \psi_{4}}{\partial t}= & C_{11 x} \frac{\partial \psi_{1}}{\partial x}+C_{12 x} \frac{\partial \psi_{2}}{\partial x}+C_{13 x} \frac{\partial \psi_{3}}{\partial x}+C_{14 x} \frac{\partial \psi_{4}}{\partial x}+ \\
= & R_{1}
\end{aligned}\right.
$$

De maneira a facilitar a implementação deste sistema de equações, tabelas que mostram os valores de cada uma das constantes acima são encontradas na tabela C.1 (apêndice C). 


\subsubsection{Entrada}

As condições fixas e calculadas nos volumes "fantasmas" das equações adjuntas são completamente análogas às do escoamento e, portanto, estão relacionadas às características que "entram" ou "saem" dele. A figura 4.1 apresenta esquematicamente a propagação de características adjuntas em fronteiras de entrada. Observe que, devido à forma como o problema dual foi formulado, elas são "espelhadas" em relação às características do problema primal. Este fato será explorado na fixação de propriedades adjuntas vindas do escoamento não-perturbado.

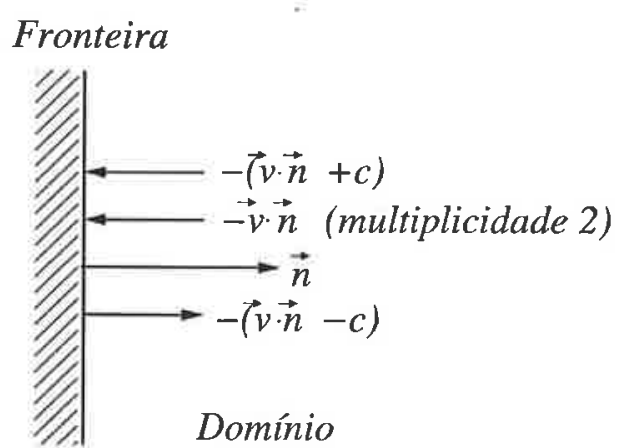

(a) Caso subsônico

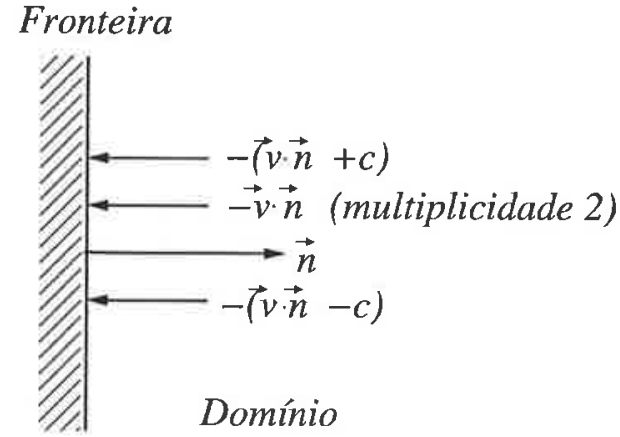

(b) Caso supersônico

Figura 4.1: Propagação de características adjuntas em fronteiras de entrada

Subsônica Neste caso, deve-se resolver 3 características $(-\overrightarrow{\mathbf{v}} \cdot \vec{n},-\overrightarrow{\mathbf{v}} \cdot \vec{n} \mathrm{e}$ $-(\overrightarrow{\mathbf{v}} \cdot \vec{n}+c))$ apresentadas no sistema de equações (4.16) abaixo:

$$
\left\{\begin{array}{l}
C_{11 t} \frac{\partial \psi_{1}}{\partial t}+C_{12 t} \frac{\partial \psi_{2}}{\partial t}+C_{13 t} \frac{\partial \psi_{3}}{\partial t}+C_{14 t} \frac{\partial \psi_{4}}{\partial t}=R_{1} \\
C_{21 t} \frac{\partial \psi_{1}}{\partial t}+C_{22 t} \frac{\partial \psi_{2}}{\partial t}+C_{23 t} \frac{\partial \psi_{3}}{\partial t}+C_{24 t} \frac{\partial \psi_{4}}{\partial t}=R_{2} \\
C_{31 t} \frac{\partial \psi_{1}}{\partial t}+C_{32 t} \frac{\partial \psi_{2}}{\partial t}+C_{33 t} \frac{\partial \psi_{3}}{\partial t}+C_{34 t} \frac{\partial \psi_{4}}{\partial t}=R_{3}
\end{array}\right.
$$

Note, no entanto, que existem 4 incógnitas e apenas 3 equações. Portanto, deve-se utilizar alguma informação de corrente não-perturbada para determinar o estado completo da fronteira. Entretanto, não existe uma propriedade conhecida à ser fixada a priori, já que as variáveis adjuntas não tem sentido físico. Esta informação deve vir da condição de contorno de farfield pela equação (3.38) reescrita abaixo:

$$
\psi_{\alpha} C_{\alpha \beta}^{(2)}\left(\delta Q_{\beta}-\delta Q_{\beta}^{*}\right)=0
$$

Observe, no entanto, que o termo:

$$
\delta Q_{\beta}^{*}=\frac{\partial Q_{\beta}}{\partial \xi^{k}} \delta \xi^{k}
$$

está relacionado com a variação da métrica e, uma vez que as mudanças da malha 
são muito pequenas no farfield, este termo pode ser desprezado. Assim, a equação que representa a condição de contorno de farfield recai na mesma expressão do gradiente completo reescrita abaixo:

$$
\psi_{\alpha} C_{\alpha \beta}^{(2)} \delta Q_{\beta}=0
$$

Este termo involve o produto escalar entre as variáveis e variações de fluxo normais à fronteira no domínio do escoamento. Na realidade, strictu sensu, as variações de fluxo normais à fronteira no domínio do escoamento são dados pela equação (3.10). Entretanto, o segundo termo desta expressão também está relacionado com variações da métrica e, como já dito diversas vezes neste trabalho, estas variações são despreziveis ao longe, uma vez que a região mais influenciada no processo de otimização é a região próxima ao aerofólio.

Assim, a anulação deste termo, implica que $\psi$ deve ser ortogonal à todas as variações de fluxo realizáveis $C_{\alpha \beta}^{(2)} \delta Q_{\beta}$. Desta forma, as variáveis $\psi$ podem ser entendidas como forças de vínculo generalizadas que impõem conservação de massa, quantidade de movimento e energia em todas as variações de fluxo em toda fronteira (VOLPE; SANTOS, 2009). Pode-se, ainda, relacioná-las ao princípio dos deslocamentos virtuais, onde as variáveis adjuntas são forças que não exercem trabalho sobre os deslocamentos virtuais, representados aqui pelas variações de fluxo realizáveis, mantendo assim o sistema em equilíbrio ou, em outras palavras, realizável (GANTMACHER, 1975). Esta é uma possível interpretação do significado das variáveis adjuntas e suas condições de contorno.

Dado o caminho que o problema variacional é construído, a mesma interpretação das variáveis adjuntas poderia, na realidade, ser empregada no domínio do escoamento. É importante recordar que $\delta Q$ deve ser realizável, mas é, de outro modo, arbitrário. Então o único meio de tornar esta integral nula é tomar $\psi$ como solução das equações adjuntas. Dentro desta estrutura, a lógica por traz do método é semelhante a minimizar o trabalho virtual das forças generalizadas de vínculo $\psi$, assim assegurando que a trajetória do sistema no espaço de estado é inteiramente realizável (VOLPE; SANTOS, 2009). Embora estas idéias não tenham qualquer relação na álgebra, elas podem guiar a derivação que segue (relações de compatibilidade).

$\mathrm{Na}$ condição de entrada subsônica, foram fixadas 3 propriedades para a resolução do escoamento: pressão de estagnação, temperatura de estagnação e o ângulo de ataque. Para poder utilizar estas informações, no entanto, é necessário reescrevê-las como variações das variáveis conservadas $Q_{\beta}$. A temperatura de 
estagnação é dada pela seguinte expressão:

$$
T_{0}=T\left(1+\frac{(\gamma-1)\left(u^{2}+v^{2}\right)}{2 c^{2}}\right)
$$

Mas, lembrando que $c=\sqrt{\gamma R T}$ e $T=\frac{p}{R \rho}$, então:

$$
T_{0}=\frac{p\left(1+\frac{(\gamma-1)\left(u^{2}+v^{2}\right)}{2 \gamma p}\right)}{R \rho}
$$

Que pode ser escrita em função das variáveis de estado $Q_{\beta}$ :

$$
T_{0}=-\frac{Q_{2}^{2}+Q_{3}{ }^{2}-\gamma\left(2 p Q_{1}+{Q_{2}}^{2}+Q_{3}{ }^{2}\right)}{2 \gamma R Q_{1}{ }^{2}}
$$

Já a pressão de estagnação é dada por:

$$
p_{0}=p\left(1+\frac{(\gamma-1)\left(u^{2}+v^{2}\right)}{2 c^{2}}\right)^{\frac{\gamma}{\gamma-1}}
$$

Mas, como:

$$
T=\frac{p}{R Q_{1}} \quad \text { e } \quad p_{0}=p\left(\frac{T_{0}}{T}\right)^{\frac{\gamma}{\gamma-1}}
$$

Então, a pressão de estagnação pode ser escrita em função das variáveis de estado $Q_{\beta}$ da seguinte maneira:

$$
p_{0}=-\frac{(\gamma-1)\left(Q_{2}^{2}+Q_{3}^{2}-2 Q_{1} Q_{4}\right)\left(1-\frac{Q_{2}^{2}+Q_{3}^{2}}{\gamma\left(Q_{2}^{2}+Q_{3}^{2}-2 Q_{1} Q_{4}\right)}\right)^{\frac{\gamma}{(\gamma-1)}}}{2 Q_{1}}
$$

Uma vez que é possível escrever a variação da temperatura de estagnação como:

$$
\delta T_{0}=\frac{\partial T_{0}}{\partial Q_{1}} \delta Q_{1}+\frac{\partial T_{0}}{\partial Q_{2}} \delta Q_{2}+\frac{\partial T_{0}}{\partial Q_{3}} \delta Q_{3}+\frac{\partial T_{0}}{\partial Q_{4}} \delta Q_{4}
$$

Então, tem-se:

$$
\begin{aligned}
\delta T_{0}= & \frac{(\gamma-1)\left[\delta Q_{4} g-(\gamma-1)\left(\delta Q_{2} u+\delta Q_{3} v\right)\right]}{\gamma R \rho}+ \\
- & \frac{(\gamma-1) \delta Q_{1}\left[e_{t} \gamma-(\gamma-1)\left(u^{2}+v^{2}\right)\right]}{\gamma R \rho}
\end{aligned}
$$

Da mesma forma, para a pressão de estagnação:

$$
\delta p_{0}=\frac{\partial p_{0}}{\partial Q_{1}} \delta Q_{1}+\frac{\partial p_{0}}{\partial Q_{2}} \delta Q_{2}+\frac{\partial p_{0}}{\partial Q_{3}} \delta Q_{3}+\frac{\partial p_{0}}{\partial Q_{4}} \delta Q_{4}
$$


Então:

$$
\begin{aligned}
\delta p_{0}= & \frac{\left[2 \delta Q_{2} u+2 \delta Q_{3} v-\delta Q_{1}\left(u^{2}+v^{2}\right)\right]\left[(\gamma-1)^{2}\left(u^{2}+v^{2}\right)-2 e_{t}(\gamma-2) \gamma\right]}{2\left[2 e_{t} \gamma-(\gamma-1)\left(u^{2}+v^{2}\right)\right]}+ \\
+ & \frac{2 \delta Q_{4}\left[2 e_{t}\left(\gamma^{2}-\gamma\right)-\left(1+\gamma^{2}-\gamma\right)\left(u^{2}+v^{2}\right)\right]\left(\frac{\gamma-1-\frac{2 e_{t}}{u^{2}+v^{2}-2 e_{t}}}{\gamma}\right)^{\frac{\gamma}{\gamma-1}}}{2\left[2 e_{t} \gamma-(\gamma-1)\left(u^{2}+v^{2}\right)\right]} \text { (4.29) }
\end{aligned}
$$

A última propriedade fixada (ângulo de ataque) pode ser escrita facilmente em função das variáveis conservadas da seguinte maneira:

$$
\tan \alpha=\frac{Q_{3}}{Q_{2}}
$$

E, como sua variação é dada por:

$$
\delta(\tan \alpha)=\frac{\partial(\tan \alpha)}{\partial Q_{2}} \delta Q_{2}+\frac{\partial(\tan \alpha)}{\partial Q_{3}} \delta Q_{3}
$$

Obtém-se:

$$
\delta(\tan \alpha)=\frac{\delta Q_{3} u-\delta Q_{2} v}{\rho u^{2}}
$$

Fazendo $\delta(\tan \alpha)=0$, que equivale a fixar o ângulo de ataque matematicamente, obtém-se:

$$
\delta Q_{3}=\frac{v}{u} \delta Q_{2}
$$

Substituindo (4.33) em (4.27), fazendo $\delta T_{0}=0$ (temperatura de estagnação fixa) e resolvendo a equação para $\delta Q_{4}$, tem-se:

$$
\delta Q_{4}=\frac{\delta Q_{2}(\gamma-1)\left(u^{2}+v^{2}\right)+\delta Q_{1} u\left[e_{t} \gamma-(\gamma-1)\left(u^{2}+v^{2}\right)\right]}{\gamma u}
$$

Agora substituindo (4.33) e (4.34) em (4.29), fazendo $\delta p_{0}=0$ (pressão de estagnação fixa) e resolvendo a equação para $\delta Q_{2}$, tem-se:

$$
\delta Q_{2}=\frac{1}{2} \delta Q_{1} u\left[2-\gamma+\gamma^{2}-\frac{2 e_{t}(\gamma-1) \gamma}{\left(u^{2}+v^{2}\right)}\right]
$$

Substituindo (4.35) em (4.34), chega-se a:

$$
\delta Q_{4}=\frac{1}{2} \delta Q_{1}\left[-2 e_{t}(\gamma-2) \gamma+(\gamma-1)^{2}\left(u^{2}+v^{2}\right)\right]
$$

Finalmente substituindo (4.35) em (4.33), tem-se:

$$
\delta Q_{3}=\frac{1}{2} \delta Q_{1} v\left[2-\gamma+\gamma^{2}-\frac{2 e_{t}(\gamma-1) \gamma}{\left(u^{2}+v^{2}\right)}\right]
$$


Assim, a imposição das propriedades: pressão de estagnação, temperatura de estagnação e ângulo de ataque na condição de entrada subsônica para a resolução das equações de Euler implica na seguinte variação do vetor de variáveis de estado:

$$
\delta Q_{\beta}=\delta \mathbf{Q}=\left\{\begin{array}{c}
\delta Q_{1} \\
\frac{u}{2}\left[2-\gamma+\gamma^{2}-\frac{2 e_{t}(\gamma-1) \gamma}{\left(u^{2}+v^{2}\right)}\right] \delta Q_{1} \\
\frac{v}{2}\left[2-\gamma+\gamma^{2}-\frac{2 e_{t}(\gamma-1) \gamma}{\left(u^{2}+v^{2}\right)}\right] \delta Q_{1} \\
\frac{1}{2}\left[-2 e_{t}(\gamma-2) \gamma+(\gamma-1)^{2}\left(u^{2}+v^{2}\right)\right] \delta Q_{1}
\end{array}\right\}
$$

Ao utilizar esta variação na equação (4.19), impõe-se à realizabilidade. Ou, em outras palavras, que a variação $\delta Q_{\beta}$ satisfaz as equações que governam o escoamento. Assim, obtém-se a seguinte equação:

$$
\begin{array}{ll}
\delta Q_{1} \quad\left\{\psi_{1}\left[2-\gamma+\gamma^{2}-\frac{2 \gamma e_{t}(\gamma-1)}{u^{2}+v^{2}}\right] \frac{\left(k_{x} u+k_{y} v\right)}{2}+\right. \\
+\quad \psi_{2}\left[\frac{\left(2-\gamma+\gamma^{2}\right) k_{x} u^{2}+2\left(1-\gamma+\gamma^{2}\right) k_{y} u v-(\gamma-1) \gamma k_{x} v^{2}}{2}+\right. \\
\left.-\quad \frac{2 \gamma e_{t}(\gamma-1)\left[2 k_{y} u v+k_{x}\left(u^{2}-v^{2}\right)\right]}{2\left(u^{2}+v^{2}\right)}\right]+ \\
+\quad \psi_{3}\left[\frac{-\gamma(\gamma-1) k_{y} u^{4}+2\left(1-\gamma+\gamma^{2}\right) k_{x} u^{3} v+2 k_{y} u^{2} v^{2}}{2\left(u^{2}+v^{2}\right)}+\right. \\
+\quad \frac{2\left(1-\gamma+\gamma^{2}\right) k_{x} u v^{3}+\left(2-\gamma+\gamma^{2}\right) k_{y} v^{4}}{2\left(u^{2}+v^{2}\right)}+ \\
+\quad \frac{\left.2 \gamma e_{t}(\gamma-1)\left[k_{y}\left(u^{2}-v^{2}\right)-2 k_{x} u v\right]\right]}{2\left(u^{2}+v^{2}\right)}+ \\
-\quad \psi_{4}\left[\frac{\left(k_{x} u+k_{y} v\right)\left[(\gamma-1)\left(u^{2}+v^{2}\right)-2 \gamma e_{t}\right]\left[\left(2-\gamma+\gamma^{2}\right)\left(u^{2}+v^{2}\right)\right]}{4\left(u^{2}+v^{2}\right)}+\right. \\
\left.\left.+\quad \frac{\left(k_{x} u+k_{y} v\right)\left[(\gamma-1)\left(u^{2}+v^{2}\right)-2 \gamma e_{t}\right]\left[-2 \gamma e_{t}(\gamma-1)\right]}{4\left(u^{2}+v^{2}\right)}\right]\right\}=0
\end{array}
$$

Para que esta equação seja verdadeira independentemente da variação de $\delta Q_{1}$, é necessário que o termo entre chaves $\{\cdots\}$ seja sempre nulo. Portanto, deve se resolver a equação $\{\cdots\}=0$ para uma das variáveis adjuntas, obtendo o seu valor em função das outras, de tal modo que a condição de ortogonalidade descrita anteriormente seja satisfeita. Aqui vale ressaltar que a escolha da variável adjunta não influencia diretamente o campo de solução. Assim, é interessante escolher àquela que resulta na expressão mais simples, facilitando a implementação numérica. Escrevendo, por exemplo, $\psi_{1}$ em função das outras, obtém-se a seguinte relação de compatibilidade: 


$$
\begin{aligned}
\psi_{1}= & -\left\{\frac{\left(2+\gamma^{2}-\gamma\right) k_{x} u^{2}+2\left(1+\gamma^{2}-\gamma\right) k_{y} u v-(\gamma-1) \gamma k_{x} v^{2}}{\left(k_{x} u+k_{y} v\right)\left[2-\gamma+\gamma^{2}-\frac{2 e_{t}(\gamma-1) \gamma}{u^{2}+v^{2}}\right]}+\right. \\
& \left.-\frac{2 e_{t}(\gamma-1) \gamma\left[2 k_{y} u v+k_{x}\left(u^{2}-v^{2}\right)\right]}{\left(k_{x} u+k_{y} v\right)\left(u^{2}+v^{2}\right)\left[2-\gamma+\gamma^{2}-\frac{2 e_{t}(\gamma-1) \gamma}{u^{2}+v^{2}}\right]}\right\} \psi_{2}+ \\
& -\left\{\frac{(\gamma-1) \gamma k_{y} u^{4}+2[1+(\gamma-1) \gamma]\left(k_{x} u^{3} v+k_{x} u v^{3}\right)+2 k_{y} u^{2} v^{2}}{\left(k_{x} u+k_{y} v\right)\left(u^{2}+v^{2}\right)\left[2-\gamma+\gamma^{2}-\frac{2 e_{t}(\gamma-1) \gamma}{u^{2}+v^{2}}\right]}+\right. \\
+ & \left.\frac{[2+(\gamma-1) \gamma] k_{y} v^{4}+2 e_{t}(\gamma-1) \gamma\left[-2 k_{x} u v+k_{y}\left(u^{2}-v^{2}\right)\right]}{\left(k_{x} u+k_{y} v\right)\left(u^{2}+v^{2}\right)\left[2-\gamma+\gamma^{2}-\frac{2 e_{t}(\gamma-1) \gamma}{u^{2}+v^{2}}\right]}\right\} \psi_{3}+ \\
+ & \left\{\frac{\left[(\gamma-1)\left(u^{2}+v^{2}\right)-2 e_{t} \gamma\right]\left(2+\gamma^{2}-\gamma\right)}{2\left[2-\gamma+\gamma^{2}-\frac{2 e_{t}(\gamma-1) \gamma}{u^{2}+v^{2}}\right]}+\right. \\
- & \left.\frac{\left[(\gamma-1)\left(u^{2}+v^{2}\right)-2 e_{t} \gamma\right]\left[2 e_{t}(\gamma-1) \gamma\right]}{2\left(u^{2}+v^{2}\right)\left[2-\gamma+\gamma^{2}-\frac{2 e_{t}(\gamma-1) \gamma}{u^{2}+v^{2}}\right]}\right\} \psi_{4}
\end{aligned}
$$

Que pode ser escrita de forma simplificada na forma:

$$
\psi_{1}=C_{1} \psi_{2}+C_{2} \psi_{3}+C_{3} \psi_{4}
$$

Note, no entanto, que o fato da solução do escoamento ser a mesma durante toda a solução das equações adjuntas implica que os termos $C_{i}$ sejam constantes no tempo, mas não no espaço. Assim, tem-se:

$$
\frac{\partial}{\partial t}\left(C_{i} \psi_{i}\right)=C_{i} \frac{\partial \psi_{i}}{\partial t} \quad \text { mas } \quad \frac{\partial}{\partial x^{j}}\left(C_{i} \psi_{i}\right) \neq C_{i} \frac{\partial \psi_{i}}{\partial x^{j}}
$$

Portanto, ao derivar a equação (4.41) em relação ao tempo, obtém-se uma relação do tipo:

$$
\frac{\partial \psi_{1}}{\partial t}-C_{1} \frac{\partial \psi_{2}}{\partial t}-C_{2} \frac{\partial \psi_{3}}{\partial t}-C_{3} \frac{\partial \psi_{4}}{\partial t}=0
$$

Desta forma, com a equação (4.43) é possível, finalmente, completar o sistema linear (4.16), resultando em:

$$
\left\{\begin{aligned}
C_{11 t} \frac{\partial \psi_{1}}{\partial t}+C_{12 t} \frac{\partial \psi_{2}}{\partial t}+C_{13 t} \frac{\partial \psi_{3}}{\partial t}+C_{14 t} \frac{\partial \psi_{4}}{\partial t}=R_{1} \\
C_{21 t} \frac{\partial \psi_{1}}{\partial t}+C_{22 t} \frac{\partial \psi_{2}}{\partial t}+C_{23 t} \frac{\partial \psi_{3}}{\partial t}+C_{24 t} \frac{\partial \psi_{4}}{\partial t}=R_{2} \\
C_{31 t} \frac{\partial \psi_{1}}{\partial t}+C_{32 t} \frac{\partial \psi_{2}}{\partial t}+C_{33 t} \frac{\partial \psi_{3}}{\partial t}+C_{34 t} \frac{\partial \psi_{4}}{\partial t}=R_{3} \\
\frac{\partial \psi_{1}}{\partial t}-C_{1} \frac{\partial \psi_{2}}{\partial t}-C_{2} \frac{\partial \psi_{3}}{\partial t}-C_{3} \frac{\partial \psi_{4}}{\partial t}=0
\end{aligned}\right.
$$


Que pode ser escrito na forma matricial da seguinte maneira:

$$
\left[\begin{array}{cccc}
C_{11 t} & C_{12 t} & C_{13 t} & C_{14 t} \\
C_{21 t} & C_{22 t} & C_{23 t} & C_{24 t} \\
C_{31 t} & C_{32 t} & C_{33 t} & C_{34 t} \\
1 & -C_{1} & -C_{2} & -C_{3}
\end{array}\right]\left\{\begin{array}{c}
\frac{\partial \psi_{1}}{\partial t} \\
\frac{\partial \psi_{2}}{\partial t} \\
\frac{\partial \psi_{3}}{\partial t} \\
\frac{\partial \psi_{4}}{\partial t}
\end{array}\right\}=\left\{\begin{array}{c}
R_{1} \\
R_{2} \\
R_{3} \\
0
\end{array}\right\}
$$

O sistema pode ser resolvido numericamente com decomposição LU, por exemplo. Então, as variações das variáveis adjuntas no volume fantasma são dadas por:

$$
\delta \psi_{i}=\frac{\partial \psi_{i}}{\partial t} \Delta t
$$

Por fim, a atualização das variáveis adjuntas em um determinado volume "fantasma" é dada por:

$$
\psi_{i}^{n+1}=\psi_{i}^{n}+\delta \psi_{i}
$$

Supersônica Neste caso, deve-se resolver as 4 características, já que todas elas saem do domínio. Assim, basta resolver diretamente o sistema de equações (4.15) para obter as variações $\delta \psi_{i}$ e assim determinar o valor das variáveis adjuntas nos volumes "fantasmas" através da expressão:

$$
\psi_{\text {ghost }}^{n+1}=\psi_{\text {ghost }}^{n}+\delta \psi
$$

A tabela 4.1 apresenta um resumo das condições de contorno adjuntas nas fronteiras de entrada que correspondem a cada regime de escoamento.

Tabela 4.1: Resumo das condições de contorno adjuntas de entrada

\begin{tabular}{ccc}
\hline \hline Regime & Variação $\delta \mathbf{Q}$ & Condição adjunta $\mathbf{\Psi}$ \\
\hline \multirow{2}{*}{ subsônico } & $\delta Q_{2}\left(\delta Q_{1}\right), \delta Q_{3}\left(\delta Q_{1}\right), \delta Q_{4}\left(\delta Q_{1}\right)$ & $\psi_{1}\left(\psi_{2}, \psi_{3}, \psi_{4}\right)$ \\
& eqs. $(4.35)-(4.37)$ & eq. $(4.40)$ \\
\hline supersônico & $\delta Q_{i}=0$ & $\psi_{i}$ livres \\
\hline \hline
\end{tabular}

\subsubsection{Saída}

A figura 4.2 apresenta esquematicamente a propagação de características adjuntas em fronteiras de saída. 


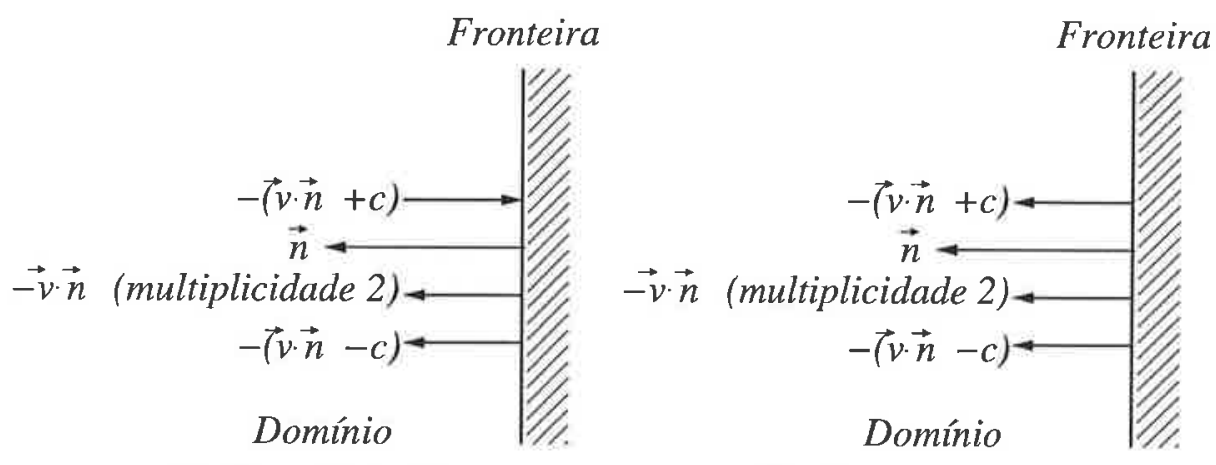

$\begin{array}{ll}\text { (a) Caso subsônico } & \text { (b) Caso supersônico }\end{array}$

Figura 4.2: Propagação de características adjuntas em fronteiras de saída

Subsônica Neste caso, 3 caraterísticas entram no domínio e apenas uma sai. Isto significa que se deve calcular a carcterística que sai do domínio $\left(\overrightarrow{v_{n}}+c\right)$.

$$
C_{31 t} \frac{\partial \psi_{1}}{\partial t}+C_{32 t} \frac{\partial \psi_{2}}{\partial t}+C_{33 t} \frac{\partial \psi_{3}}{\partial t}+C_{34 t} \frac{\partial \psi_{4}}{\partial t}=R_{3}
$$

Novamente, ocorre um problema para se resolver as equações características. Neste caso há apenas 1 equação e 4 incógnitas. Para determinar relações de compatibilidade análogas à condição de entrada subsônica, deve-se lembrar que a condição de saída subsônica do escoamento fixa a pressão estática, que é escrita como:

$$
p=(\gamma-1) \rho\left(e_{t}+\frac{\left(-u^{2}-v^{2}\right)}{2}\right)
$$

Ou ainda, em função das variáveis conservadas:

$$
p=(\gamma-1)\left(Q_{4}-\frac{Q_{2}^{2}}{2 Q_{1}}-\frac{Q_{3}^{2}}{2 Q_{1}}\right)
$$

Assim, sua variação pode ser escrita como:

$$
\delta p=\frac{\partial p}{\partial Q_{1}} \delta Q_{1}+\frac{\partial p}{\partial Q_{2}} \delta Q_{2}+\frac{\partial p}{\partial Q_{3}} \delta Q_{3}+\frac{\partial p}{\partial Q_{4}} \delta Q_{4}
$$

Calculando as derivadas $\partial p / \partial Q_{\beta}$ à partir da expressão (4.51) e substituindo as na equação (4.52), obtém-se a variação $\delta p$ em função, apenas, das variáveis conservadas $Q_{\beta}$ :

$$
\delta p=(\gamma-1)\left[\left(\frac{Q_{2}^{2}}{2 Q_{1}^{2}}+\frac{Q_{3}^{2}}{2 Q_{1}^{2}}\right) \delta Q_{1}-\frac{Q_{2}}{Q_{1}} \delta Q_{2}-\frac{Q_{3}}{Q_{1}} \delta Q_{3}+\delta Q_{4}\right]
$$

Impondo $\delta p=0$, que nada mais é do que a condição de contorno física imposta 
na saída subsônica do problema primal, obtém-se a seguinte relação:

$$
\delta Q_{4}=u \delta Q_{2}+v \delta Q_{3}-\frac{\left(u^{2}+v^{2}\right)}{2} \delta Q_{1}
$$

Assim, a variação do vetor de estados que corresponde a imposição de $\delta p=0$ é dada por:

$$
\delta \mathbf{Q}=\left\{\begin{array}{c}
\delta Q_{1} \\
\delta Q_{2} \\
\delta Q_{3} \\
u \delta Q_{2}+v \delta Q_{3}-\frac{\left(u^{2}+v^{2}\right)}{2} \delta Q_{1}
\end{array}\right\}
$$

Naturalmente, existem três graus de liberdade livres e, de cada um deles, obter-se-á uma relação de compatibilidade.

Utilizando, então esta variação na equação (4.19), é possível obter uma relação que satisfaz a condição de ortogonalidade já descrita. Assim:

$$
\begin{aligned}
& \delta Q_{1}\left\{\left(k_{x} u+k_{y} v\right)\left(-u \psi_{2}-v \psi_{3}\right)+\right. \\
+ & \left.\frac{\left(k_{x} u+k_{y} v\right)}{2} \psi_{4}\left[(\gamma-2)\left(u^{2}+v^{2}\right)-2 \gamma e_{t}\right]\right\}+ \\
+ & \delta Q_{2}\left\{k_{x}\left(\psi_{1}+u \psi_{2}+v \psi_{3}\right)+\psi_{2}\left(k_{x} u+k_{y} v\right)+\right. \\
+ & \left.\psi_{4}\left[k_{x} \gamma e_{t}+u\left(k_{x} u+k_{y} v\right)+\frac{(\gamma-1) k_{x}\left(u^{2}+v^{2}\right)}{2}\right]\right\}+ \\
+ & \delta Q_{3}\left\{k_{y}\left(\psi_{1}+u \psi_{2}+v \psi_{3}\right)+\psi_{3}\left(k_{x} u+k_{y} v\right)+\right. \\
+ & \left.\psi_{4}\left[k_{y} \gamma e_{t}+v\left(k_{x} u+k_{y} v\right)-\frac{(\gamma-1) k_{y}\left(u^{2}+v^{2}\right)}{2}\right]\right\}=0
\end{aligned}
$$

Portanto, a única forma de satisfazer a equação (4.19) garantindo uma perturbação arbitrária $\delta \mathrm{Q}$ é fazendo cada um dos termos entre chaves $(\{\cdots\})$ iguais a zero. O que resulta nas seguintes relações de compatibilidade:

$$
\begin{aligned}
& \psi_{1}=\frac{1}{2}\left[2 e_{t} \gamma-(\gamma-1)\left(u^{2}+v^{2}\right)\right] \psi_{4}=C_{1} \psi_{4} \\
& \psi_{2}=\left\{\frac{-2 e_{t} \gamma k_{x}+(\gamma-2) k_{x} u^{2}-2 k_{y} u v+\gamma k_{x} v^{2}}{2\left(k_{x} u+k_{y} v\right)}\right\} \psi_{4}=C_{2} \psi_{4} \\
& \psi_{3}=\left\{\frac{-2 e_{t} \gamma k_{y}+(\gamma-2) k_{y} v^{2}-2 k_{x} u v+\gamma k_{y} u^{2}}{2\left(k_{x} u+k_{y} v\right)}\right\} \psi_{4}=C_{3} \psi_{4}
\end{aligned}
$$

Substituindo as relações de compatibilidade (4.57)-(4.59) em (4.49), tem-se:

$$
\frac{\partial \psi_{4}}{\partial t}=\frac{R_{3}}{C_{31 t} C_{1}+C_{32 t} C_{2}+C_{33 t} C_{3}+C_{34 t}}
$$

As demais derivadas temporais podem ser obtidas pela derivação no tempo 
das equações (4.57)-(4.59), que resultam em:

$$
\begin{aligned}
\frac{\partial \psi_{1}}{\partial t} & =C_{1} \frac{\partial \psi_{4}}{\partial t} \\
\frac{\partial \psi_{2}}{\partial t} & =C_{2} \frac{\partial \psi_{4}}{\partial t} \\
\frac{\partial \psi_{3}}{\partial t} & =C_{3} \frac{\partial \psi_{4}}{\partial t}
\end{aligned}
$$

Então, as variações $\delta \psi_{i}$ são obtidas através da relação:

$$
\delta \psi_{i}=\frac{\partial \psi_{i}}{\partial t} \Delta t
$$

Finalmente, a atualização das variáveis nos volumes "fantasmas" são dadas por:

$$
\psi_{i}^{n+1}=\psi_{i}^{n}+\delta \psi_{i}
$$

Supersônica Neste caso, todas as 4 características entram no domínio e, portanto, não se resolve nenhuma característica. Assim:

$$
\psi_{i}^{n+1}=\psi_{i}^{n}=\cdots=\psi_{i}^{0}=0
$$

Note que, a condição homogênea é suficiente para anular o termo de condição de contorno de farfield, quaisquer que sejam as variações $\delta Q_{\beta}$.

A tabela 4.2 apresenta um resumo das condições de contorno adjuntas nas fronteiras de saída que correspondem a cada regime de escoamento.

Tabela 4.2: Resumo das condições de contorno adjuntas de saída

\begin{tabular}{ccc}
\hline \hline Regime & Variação $\delta \mathbf{Q}$ & Condição adjunta $\mathbf{\Psi}$ \\
\hline \multirow{2}{*}{ subsônico } & $\begin{array}{c}\delta Q_{4}\left(\delta Q_{1}, \delta Q_{2}, \delta Q_{3}\right) \\
\text { eq. }(4.54)\end{array}$ & $\begin{array}{c}\psi_{1}\left(\psi_{4}\right), \psi_{2}\left(\psi_{4}\right), \psi_{3}\left(\psi_{4}\right) \\
\text { eqs. }\end{array}$ \\
\hline supersônico & $\delta Q_{i}$ livres & $\psi_{i}=0$ \\
\hline \hline
\end{tabular}




\section{PARAMETRIZAÇÃo DA GeOMETRIA}

De maneira geral, aerofólios tem características geométricas bastante complexas como inclinação do bordo de ataque e segunda derivada do bordo de fuga infinitas (KULFAN; BUSSOLETTI, 2006). Conseqüentemente, para descrevê-lo numericamente são necessários, tipicamente, muitos parâmetros de controle. Neste trabalho, adotou-se a parametrização proposta por Kulfan e Bussoletti (2006) para superfícies aerodinâmicas devido às suas promissoras vantagens no processo de otimização de aerofólios através de uma representação simples e robusta com apenas poucos parâmetros escalares que capturam um grande espaço de projeto para otimização e análise de projetos aerodinâmicos. Neste capítulo será feita uma breve descrição desta parametrização, uma vez que o seu estudo não é o foco deste trabalho. Para maiores detalhes, o leitor pode reportar-se ao artigo original ou ao trabalho de Ceze (2008).

\subsection{Descrição Matemática da Geometria do Aero- fólio}

A parametrização CST $^{1}$ proposta por Kulfan e Bussoletti (2006) leva em consideração características fundamentais para um aerofólio imerso em um escoamento subsônico como a curvatura do bordo de ataque e a forma do bordo de fuga. Sua formulação geral é dada pela seguinte expressão:

$$
\frac{y}{\bar{c}}(x / \bar{c})=\mathcal{C}(x / \bar{c}) \cdot S(x / \bar{c})+\frac{x}{\bar{c}} \cdot \frac{\Delta y_{T E}}{\bar{c}}
$$

onde, $\Delta y_{T E}$ é a espessura do bordo de fuga, o termo $S(x / \bar{c})$ representa a função de forma e $\mathcal{C}(x / \bar{c})$ a função de classe, definida de forma geral como:

$$
\mathcal{C}(x / \bar{c})=\left(\frac{x}{\bar{c}}\right)^{N 1} \cdot\left(1-\frac{x}{\bar{c}}\right)^{N 2}
$$

\footnotetext{
${ }^{1}$ sigla em inglês para Class-Shape function Transformation
} 
Kulfan e Bussoletti (2006) mostram, ainda, que os valores dos exponentes $N 1$ e $N 2$ definem classes gerais básicas de formas geométricas definidas pela expressão (5.1). Para um aerofólio, por exemplo, os valores adotados são $N 1=1 / 2 \mathrm{e}$ $N 2=1$. O primeiro garante um bordo de ataque arredondado, enquanto o segundo é responsável pelo bordo de fuga formar um ângulo agudo. A grande idéia desta parametrização é que cada combinação dos valores destes expoentes correspondem à um tipo de forma aerodinâmica, independentemente da função de forma adotada.

Além disso, o valor da função de forma nos extremos $\frac{x}{\bar{c}}=0$ e $\frac{x}{\bar{c}}=1$ estão diretamente relacionados com o raio de curvatura do bordo de ataque $\left(R_{L E}\right)$, ângulo de fechamento $(\beta)$ e espessura do bordo de fuga $\left(\Delta y_{T E}\right)$ pelas relações:

$$
S(0)=\sqrt{2 \frac{R_{L E}}{\bar{c}}} \quad \text { e } \quad S(1)=\tan \beta+\frac{\Delta y_{T E}}{\bar{c}}
$$

Em princípio, a função de forma "S" pode ser arbitrária. Pode se escolher, por exemplo, uma simples função unitária $S(x / \bar{c})=1$. Ceze (2008) compara a utilização de pontos de controle de uma curva spline com polinômios de Bernstein propostos por Kulfan e Bussoletti (2006) para parametrizá-la. A definição de um polinômio de Bernstein de ordem $n, B P n$, é:

$$
B P n=\sum_{r=0}^{n} K_{r, n} z^{r}(1-z)^{n-r} \quad \text { com } \quad K_{r, n} \equiv\left(\begin{array}{c}
n \\
r
\end{array}\right) \equiv \frac{n !}{r !(n-r) !}
$$

onde a variável $z$ é limitada a valores entre 0 e 1 .

A função de forma é definida à partir destes polinômios através da introdução de parâmetros de projeto $a_{k_{i}}$ pela expressão:

$$
S(x / \bar{c})=\sum_{i=0}^{n}\left[a_{k_{i}} \cdot K_{i, n} \cdot\left(\frac{x}{\bar{c}}\right)^{i} \cdot\left(1-\frac{x}{\bar{c}}\right)^{n-i}\right]
$$

Ceze (2008) descreve características obtidas, com a utilização dos polinômios de Bernstein, como continuidade de segunda ordem e pouca sensibilidade à perturbações bruscas das variáveis de projeto como desejáveis em aplicações aerodinâmicas, justificando a escolha feita por Kulfan e Bussoletti (2006).

\subsection{Propriedades da Parametrização CST}

Ceze (2008) faz um estudo deste tipo de parametrização e conclui que quanto maior o número de parâmetros que representam a geometria, pior é o condicio- 
namento da matriz dos coeficientes do sistema de equações que relaciona pontos de controle da geometria com os parâmetros. Isto significa que podem haver combinações diferentes de parâmetros que resultam em geometrias muito parecidas, que são computacionalmente iguais dentro de uma certa tolerância. Assim, esta "não-unicidade" da parametrização CST pode dificultar a convergência do algorítimo de busca do mínimo da função de mérito.

Para representar uma determinada geometria com a parametrização CST, minimiza-se o erro da geometria desejada e a obtida pela parametrização pelo método dos mínimos quadrados. Uma vez que a principal aplicação do método adjunto visada neste trabalho é a otimização de aerofólios sujeitos a escoamentos transônicos, é natural escolher uma geometria usada nestas condições para analisar o erro da representação com relação ao número de parâmetros utilizado. Assim, a figura 5.1 compara duas representações do perfil RAE 2822. Note que parametrizá-la com grau 1 não é suficiente para uma boa representação da geometria, entretanto, com uma parametrização de grau 7 já é possível capturar bem suas características (CHIEREGATTI, 2008).

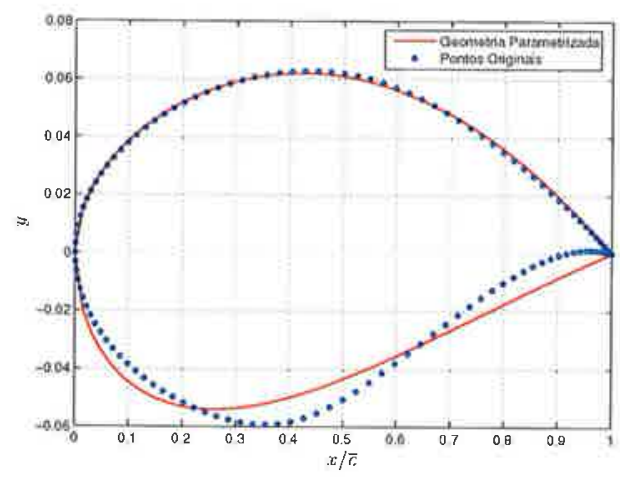

(a) Grau 1

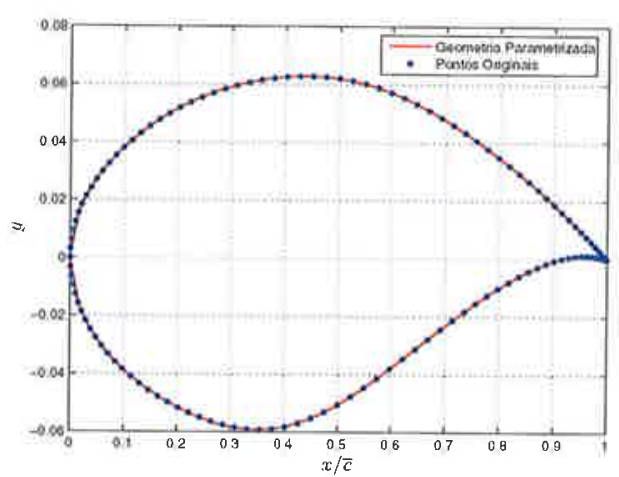

(b) Grau 7

Figura 5.1: Comparação da parametrização com diferentes graus de aproximação

A figura 5.2 apresenta um gráfico do erro da representação em relação a geometria exata em função do número de parâmetros utilizados. Este gráfico semilogarítimico tem como objetivo ilustrar a melhora do desempenho da parametrização com o aumento do número de parâmetros.

Observe que, neste caso, à partir da utilização de 5 parâmetros, para representar o intra ou extradorso de um aerofólio, já não existem ganhos significativos em qualidade da representação. Aliando este fato às observações feitas por Ceze (2008) com relação à "não-unicidade" da parametrização, pode-se chegar à um consenso de que a representação de aerofólios apropriados para o regime de vôo 
transônico, como o RAE 2822, durante o processo de otimização deve utilizar algo entre 5 e 10 parâmetros. Por este motivo, nos testes de otimização apresentados no capítulo 6, as geometrias são representadas com 7 parâmetros.

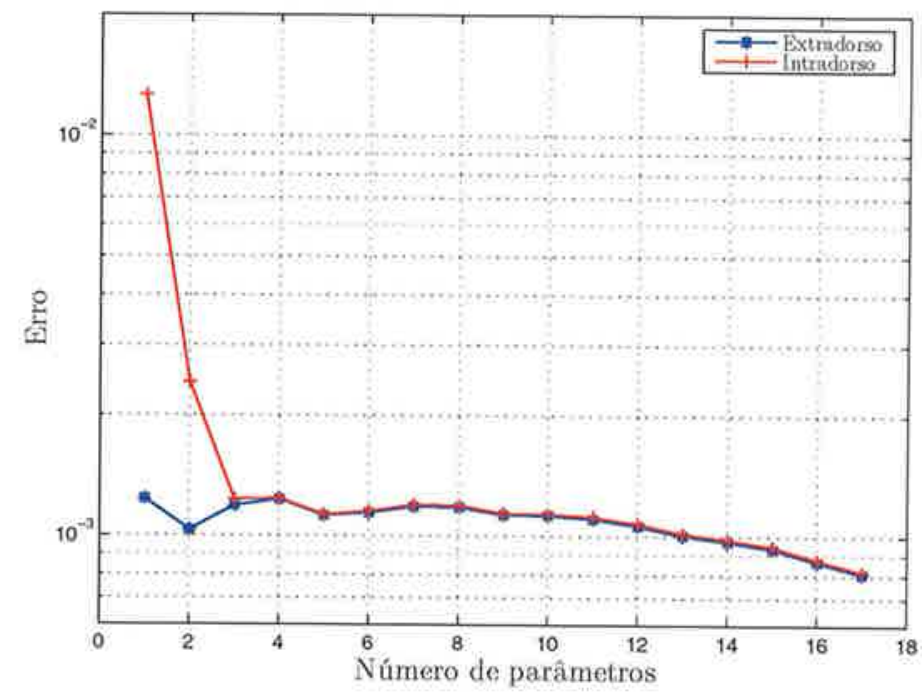

Figura 5.2: Erro da parametrização com relação ao número de parâmetros 


\section{Resultados E Discussões}

Os resultados apresentados neste capítulo são divididos em casos de validação e aplicação. Como já destacado na seção 1.2, um mesmo algorítimo de solução das equações adjuntas pode ser construído de modo a permitir a implementação de várias medidas de mérito. Assim, ao menos em tese, é possível validar esse algorítimo com base numa medida de mérito específica, para só então desenvolver as demais.

Uma vez que as variáveis adjuntas não possuem um significado físico, não existem maneiras de validar o código computacional implementado para determinálas apenas à partir de seu campo de solução. Contudo, isto pode ser realizado através de casos de validação que utilizam medidas de mérito apropriadas, como as de projeto inverso. Em essência, elas implicam na adoção de distribuições de pressão objetivo que correspondem a geometrias conhecidas previamente, sob as mesmas condições do escoamento da solução base. Com isso é possível verificar se a rotina é capaz de recuperar a geometria que, de fato, corresponde à distribuição objetivo prescrita. Naturalmente, para que os testes sejam efetivos é preciso que as distribuições objetivo representem resultados experimentais ou, pelo menos, resultados numéricos validados experimentalmente.

Já os casos de aplicação são problemas de otimização aerodinâmica com interesse mais prático na indústria e correspondem a casos em que se procura obter alguma melhora no desempenho de uma geometria inicial, como a maximização da sustentação. No entanto, uma melhora no desempenho também pode ser obtida por meio de medidas de mérito de projeto inverso ao editar uma distribuição de pressão objetivo que apresente características desejadas. É importante notar que nestes casos não se conhece a geometria que corresponde à distribuição de pressão desejada a priori e nem ao menos se tal distribuição é factível.

Todos os casos apresentados neste capítulo envolvem medidas de mérito de projeto inverso. Além disso, foram simulados com as seguintes condições de escoamento não-perturbado: $p_{\infty}=108987.7728 \mathrm{~Pa}$ e $T_{\infty}=255.556 \mathrm{~K}$, com razão de calores específicos $\gamma=1.4$ e constante do gás $R=287 \mathrm{~J} / \mathrm{Kg} \cdot \mathrm{K}$. 
O processo de otimização é realizado no ciclo de projeto apresentado na seção 1.3. Em todos os casos apresentados neste trabalho foram computados 50 ciclos. Este elevado número se justifica pela escolha do algorítimo de busca do mínimo escolhido, o steepest descent, que consiste variar cada um dos parâmetros $\mathcal{B}_{i}$ que determinam a geometria através de um passo fixo $\lambda$ à partir da equação (1.7), utilizando, para isso, o gradiente de sensibilidade calculado a partir das soluções adjunta e do escoamento. Como já dito, este método é pouco eficiente. Entretanto, a possibilidade de obter soluções que oscilem em torno da distribuição objetivo, principalmente em casos onde existe onda de choque, fez com que se adotasse um passo $\lambda$ pequeno.

Vale lembrar que são utilizados 7 parâmetros para representar tanto o intra quanto o extradorso do aerofólio em uma malha com 150 elementos em cada um deles. Para ilustrar a eficiência do método adjunto no processo de otimização de aerofólios em casos como estes basta lembrar que, para se obter o gradiente de sensibilidade por diferenças finitas seriam necessárias uma solução base mais uma solução para a variação de cada um dos parâmetros, ou seja, 15 soluções do escoamento. Por outro lado, com o método adjunto, bastam duas soluções de custo computacional similar: uma do escoamento e outra das equações adjuntas.

A seção 6.1 ainda apresenta discussões sobre algumas particularidades observadas na solução adjunta na superfície do aerofólio. Por fim, a seção 6.3 exibe alguns comentários sobre aspectos importantes a respeito do sentido de propagação de perturbações nos problemas primal e dual.

\subsection{Casos de Validação}

Dois casos de validação, descritos na tabela 6.1, são apresentados nesta seção. Naturalmente, ambos têm distribuições de pressão objetivo que correspondem a geometrias conhecidas.

Tabela 6.1: Casos de validação do ciclo de projeto inverso

\begin{tabular}{ccccc}
\hline \hline Caso & Geometria original & Geometria objetivo & Mach & Angulo de ataque \\
\hline 1 & RAE 2822 & NACA 0012 & 0.75 & 0.0 \\
2 & NACA 0012 & RAE 2822 & 0.75 & 1.0 \\
\hline \hline
\end{tabular}

\section{Caso de Validação 1}

A figura 6.1 apresenta um caso em que parte-se de uma geometria NACA 
0012, com distribuição de pressão desejada correspondente ao perfil RAE 2822 sujeito a um escoamento à Mach 0.75 e $0.0^{\circ}$ de ângulo de incidência. Notase que, após 50 ciclos, a geometria não coincide exatamente com o RAE 2822, entretanto o método é baseado nas diferenças entre as distribuições de pressão, que são bastante condizentes, como mostra a figura 6.1(a).

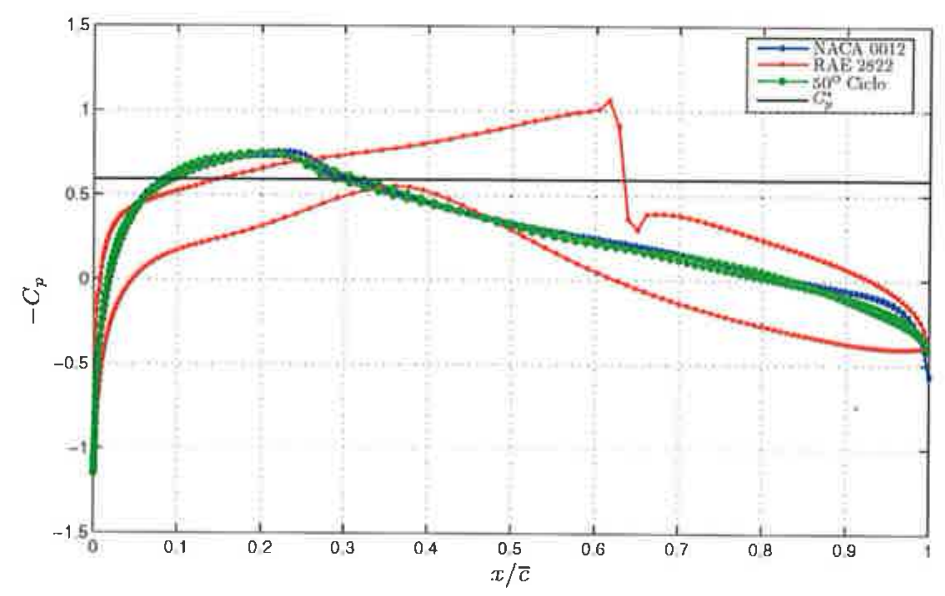

(a) Evolução de $C_{p}$

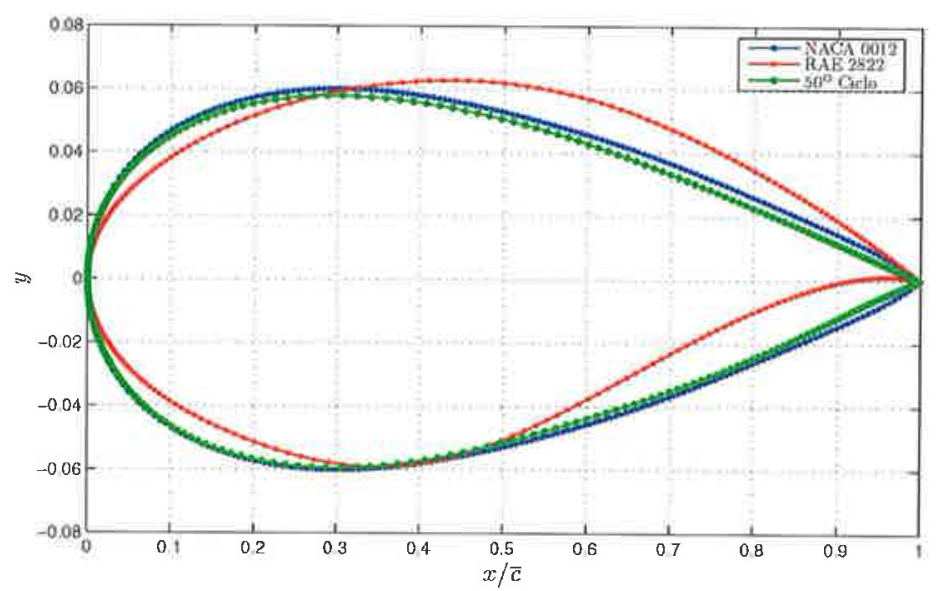

(b) Evolução da geometria

Figura 6.1: Caso de validação 1 - RAE 2822 para NACA 0012

De maneira a avaliar quantitativamente a melhora da distribuição de pressão da geometria obtida em relação a geometria inicial é interessante apresentar a evolução da medida de mérito, calculada pela equação (1.1), reapresentada abaixo por conveniência. A medida de mérito da geometria inicial é de $I_{0}=0.0151 \mathrm{e}$, após os 50 ciclos, obteve-se uma geometria que satisfaz muito bem a distribuição objetivo com $I_{50}=9.9948 \times 10^{-4}$. Vale lembrar que tanto as pressões $p$ e $p_{d}$ são os adimensionais utilizados na método numérico apresentados na seção 2.2 .

$$
I=\frac{1}{2} \int_{B_{w}}\left(p-p_{d}\right)^{2} \mathrm{~d} s
$$


Este caso apresenta uma característica muito importante, que é a recuperação da simetria. Uma vez que extra e intradorso são parametrizados separadamente, um caso em que parte-se de uma geometria assimétrica para uma geometria objetivo simétrica pode indicar falhas isoladas na implementação do código.

A figura 6.2 apresenta o campo de solução das variáveis adjuntas do primeiro ciclo de projeto do caso de validação 1. Assim, elas correspondem à solução adjunta do perfil RAE 2822 sob as condições de escoamento já citadas. Observe que o campo adjunto é não-nulo apenas numa região muito próxima da superfície sólida. Este fato justifica as condições de contorno homogêneas recomendadas na literatura para fronteiras de farfield.

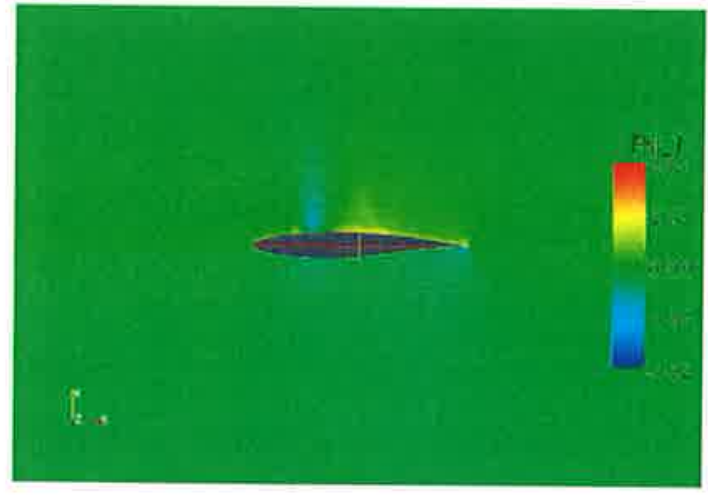

(a) $\psi_{1}$

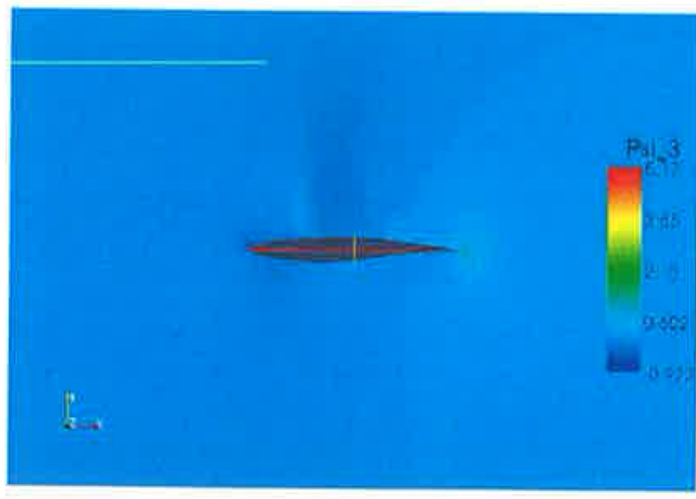

(c) $\psi_{3}$

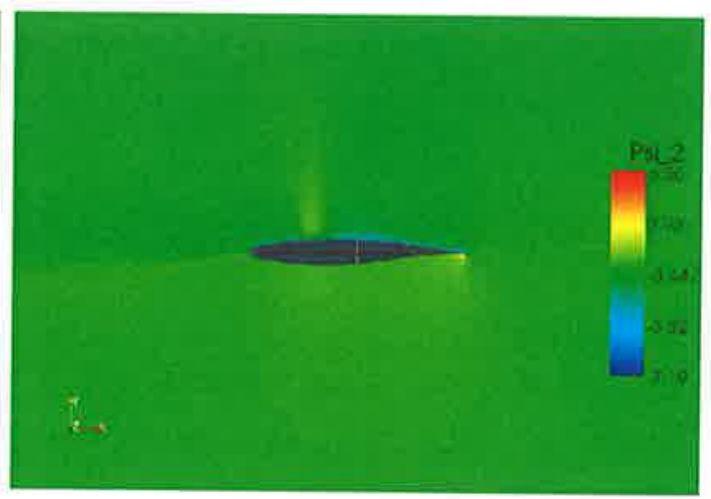

(b) $\psi_{2}$

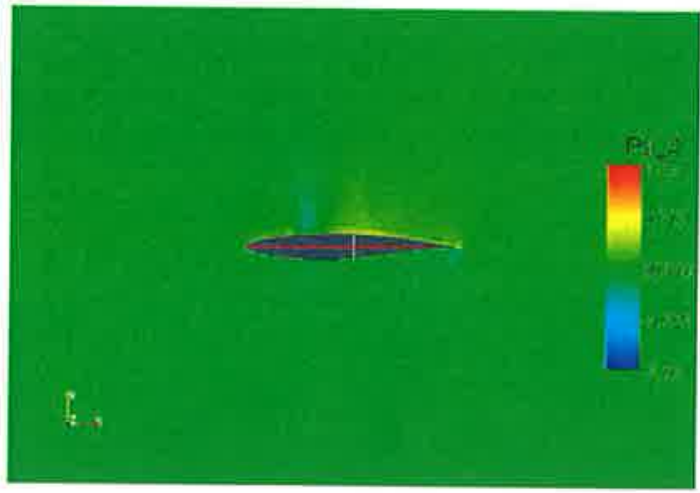

(d) $\psi_{4}$

Figura 6.2: Campo de solução adjunta do caso de validação 1

De maneira a melhorar a apresentação da solução adjunta, a figura 6.3 mostra as distribuições de pressão das geometrias inicial e objetivo juntamente com a solução adjunta na superfície do aerofólio. Note que apesar da condição periódica aplicada à linha de corte, aparentemente, existe uma descontinuidade das variáveis adjuntas no bordo de fuga do aerofólio.

Observa-se que existem características interessantes a serem analisadas no extradorso do aerofólio. Ao contrário do intradorso onde, aparentemente nada de especial ocorre (com exceção do bordo de fuga), percebem-se variações no 


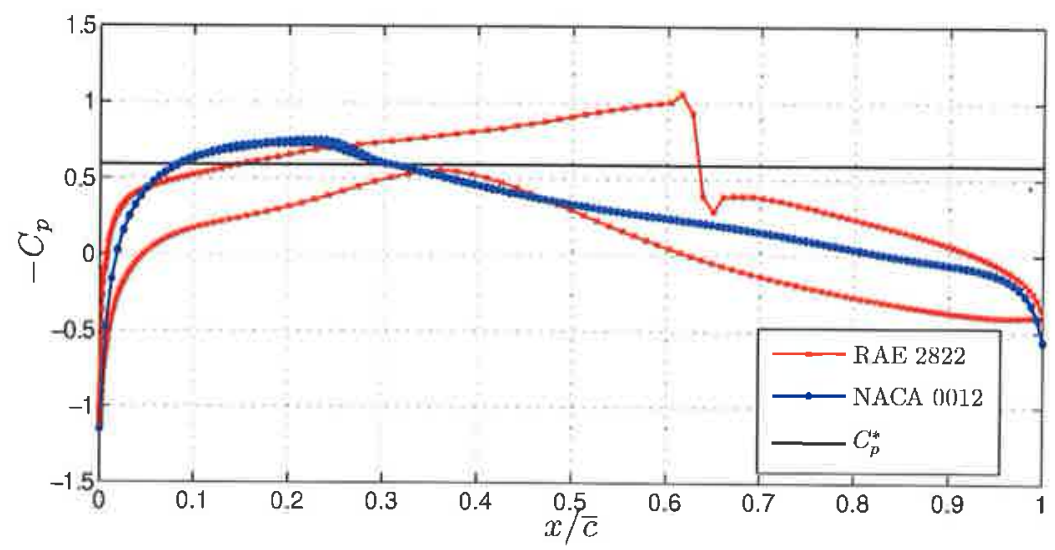

(a) Distribuição de $C_{p}$ do caso simulado

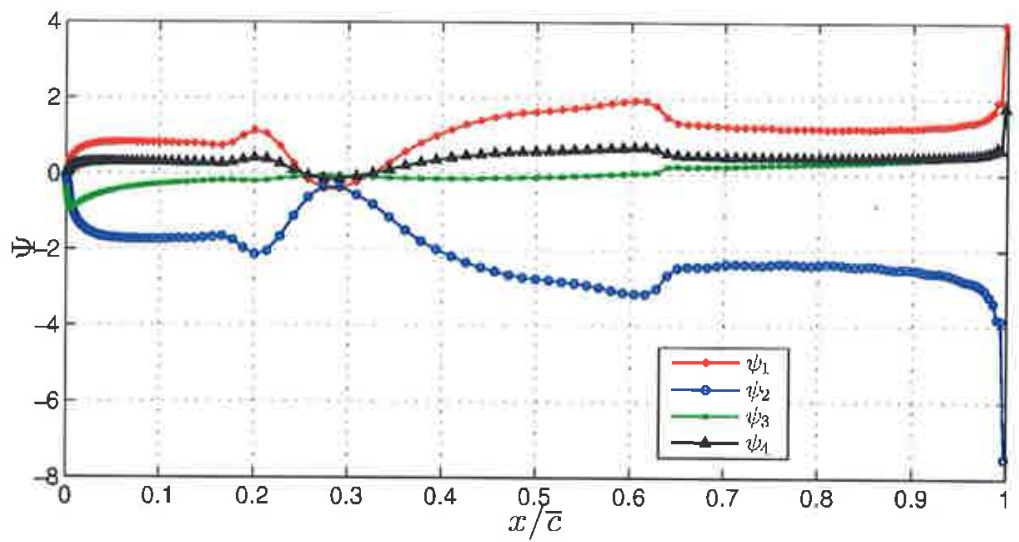

(b) Solução adjunta (extradorso)

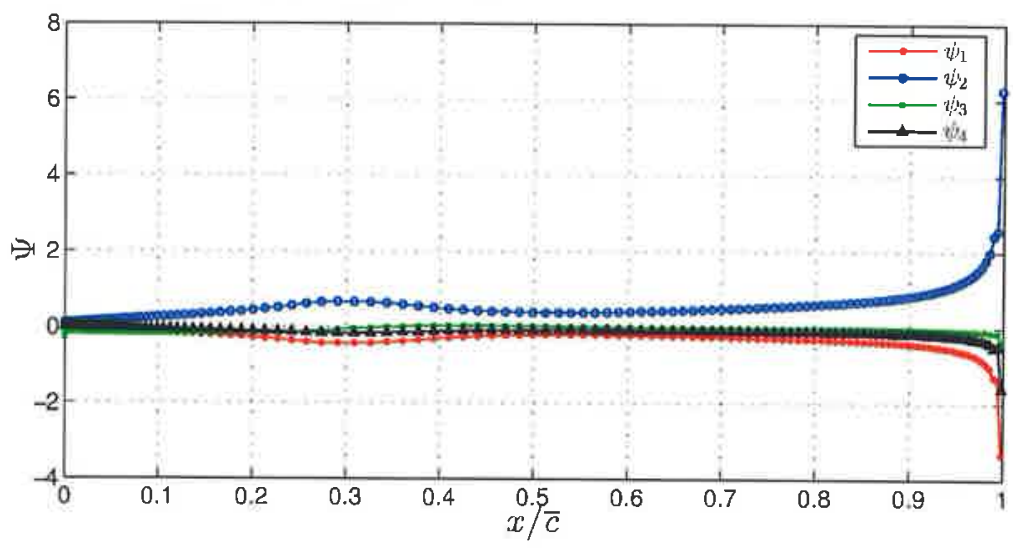

(c) Solução adjunta (intradorso)

Figura 6.3: Solução adjunta do caso de validação 1

valor das variáveis adjuntas logo após a linha sônica (onde a curva de $C_{p}$ cruza com $C_{p}^{*}$ ), no choque e nos pontos de estagnação do bordo de ataque e de fuga do aerofólio. Vale ressaltar que, na linha sônica um dos autovalores do problema adjunto é nulo, enquanto nos pontos de estagnação dois autovalores são nulos, implicando em uma singularidade do problema.

É importante destacar que as variáveis adjuntas não tem nenhum significado físico. Assim, é difícil analisar seus resultados. Contudo, um importante tópico a ser analisado diz respeito à dissipação artificial adicionada ao método. Como 
o principal objetivo deste estudo é o tratamento das condições de contorno, simplesmente adotou-se os termos dissipativos propostos na literatura. Acredita-se, no entanto, que a análise das regiões onde a dissipação numérica age no contorno da superfície sólida possa revelar importantes propriedades das variáveis adjuntas, inclusive sobre o seu comportamento nas ondas de choque, tópico já discutido por Volpe e Santos (2009) em escoamentos quase-unidimensionais.

O estudo destas características da solução adjunta e sua origem foge do escopo deste trabalho, mas são, certamente, assuntos interessantes a serem estudados em futuras atividades de pesquisa.

\section{Caso de Validação 2}

A figura 6.4, por sua vez, consiste em um teste onde a geometria inicial é o RAE 2822 e a distribuição de pressão desejada corresponde a um escoamento à Mach 0.75 e $1.0^{\circ}$ de ângulo de incidência em um perfil NACA 0012.

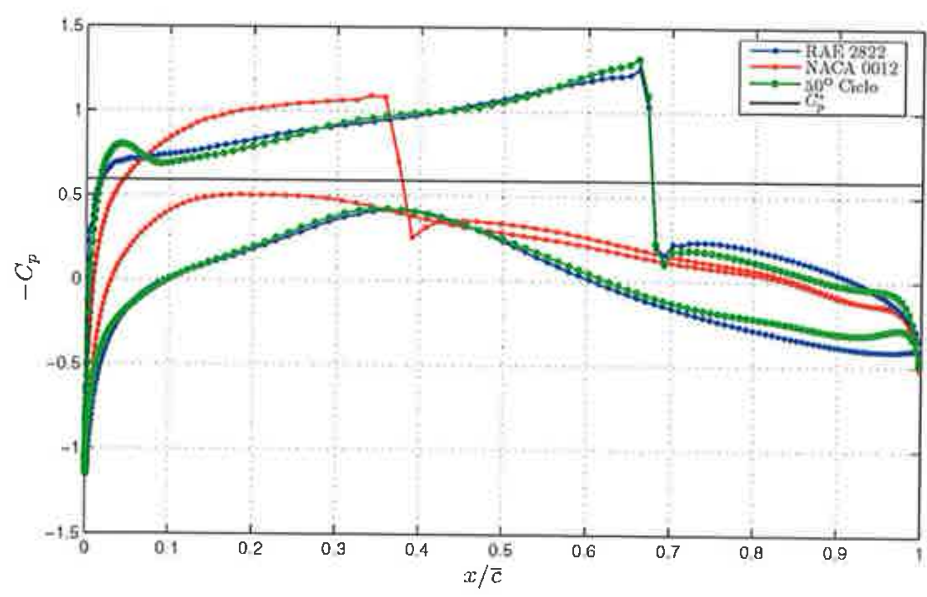

(a) Evolução de $C_{p}$

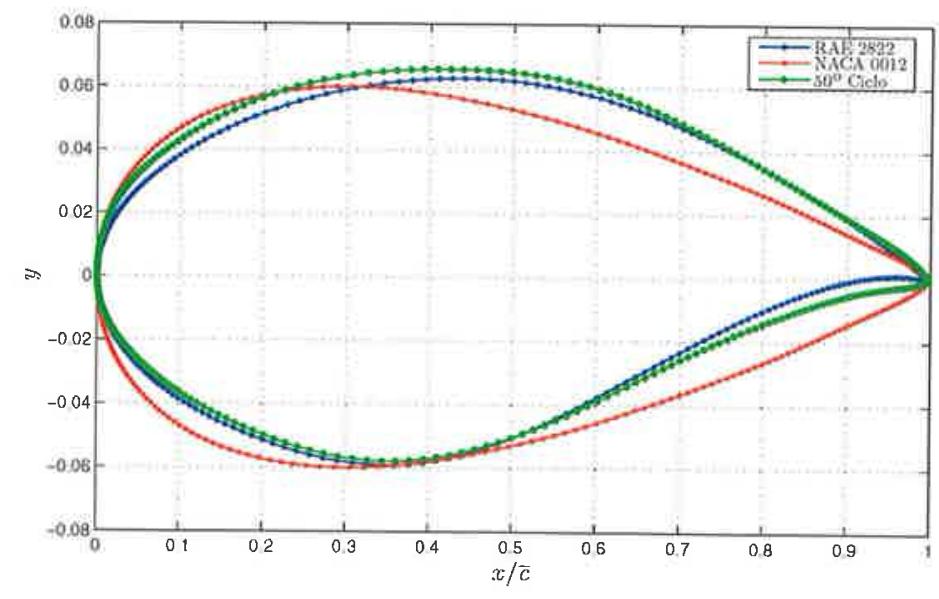

(b) Evolução da geometria

Figura 6.4: Caso de validação 2 - NACA 0012 para RAE 2822 
Nota-se que o resultado obtido é bastante consistente com a distribuição desejada, acertando o posicionamento e intensidade do choque da geometria final. Observa-se que, assim como no caso de validação 1 , a geometria final não coincide exatamente com a desejada (RAE 2822). Entretanto, a distribuição de pressão é muito próxima da desejada. A medida de mérito da geometria inicial é de $I_{0}=0.0219$ e, após os 50 ciclos, obteve se uma geometria com $I_{50}=0.0027$.

Apesar de não ser um caso prático, já que a intensidade da onda de choque na geometria final é maior do que na inicial, este caso mostra, a capacidade do método lidar com ondas de choque quando formulado com base nas equações de Euler, que apesar de representarem um modelo simplificado do escoamento, são capazes de modelar estes fenômenos não-lineares.

\subsection{Casos de Aplicação}

Assim como nos casos de validação, dois casos de aplicação são apresentados nesta seção. A tabela 6.2 descreve a geometria inicial e as condições do escoamento não-perturbado para ambos os casos.

Tabela 6.2: Casos de aplicação do ciclo de projeto inverso

\begin{tabular}{cccc}
\hline \hline Caso & Geometria original & Mach & Ângulo de ataque \\
\hline 1 & RAE 2822 & 0.75 & 0.0 \\
2 & RAE 2822 & 0.75 & 1.0 \\
\hline \hline
\end{tabular}

\section{Caso de Aplicação 1}

A figura 6.5 apresenta o resultado de um caso de aplicação do método adjunto. O escoamento incide sobre o aerofólio à Mach 0.75 e $0.0^{\circ}$ de ângulo de incidência. A distribuição desejada foi editada manualmente, tendo como objetivo aumentar a sustentação do aerofólio e eliminar a onda de choque presente sob estas condições.

Observe que, o processo de otimização não conseguiu eliminar a onda de choque. Entretanto, não há como garantir que a distribuição de pressão objetivo seja realizável. Note, no entanto, que a geometria obtida no $50^{\circ}$ ciclo conseguiu capturar bem o pico de sucção e a distribuição de pressão no intradorso, minimizando a medida de mérito e aumentando a sustentação do aerofólio sob as mesmas condições de vôo. A medida de mérito da geometria inicial é de $I_{0}=0.0079 \mathrm{e}$, após os 50 ciclos, obteve-se uma geometria com distribuição de pressão que cor- 


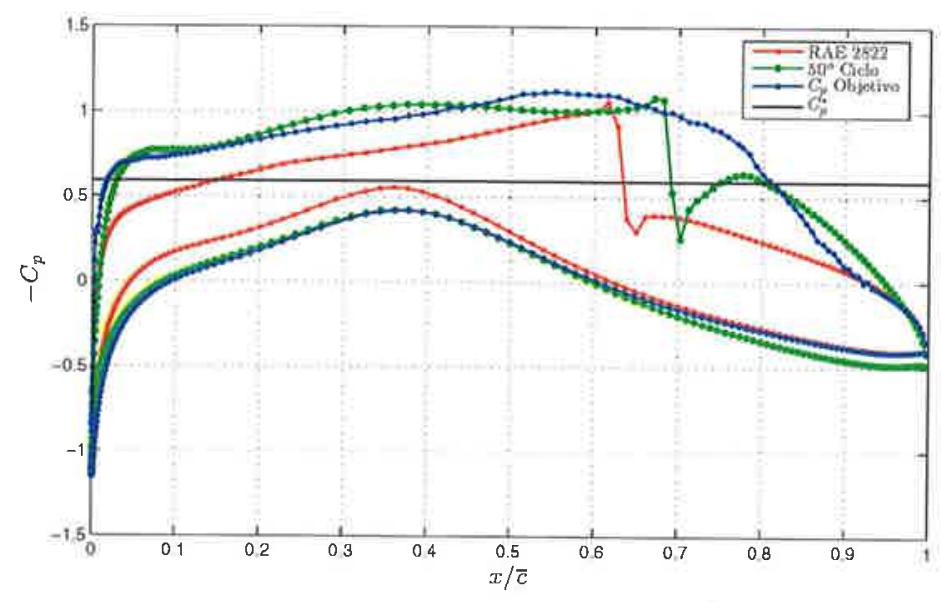

(a) Evolução de $C_{p}$

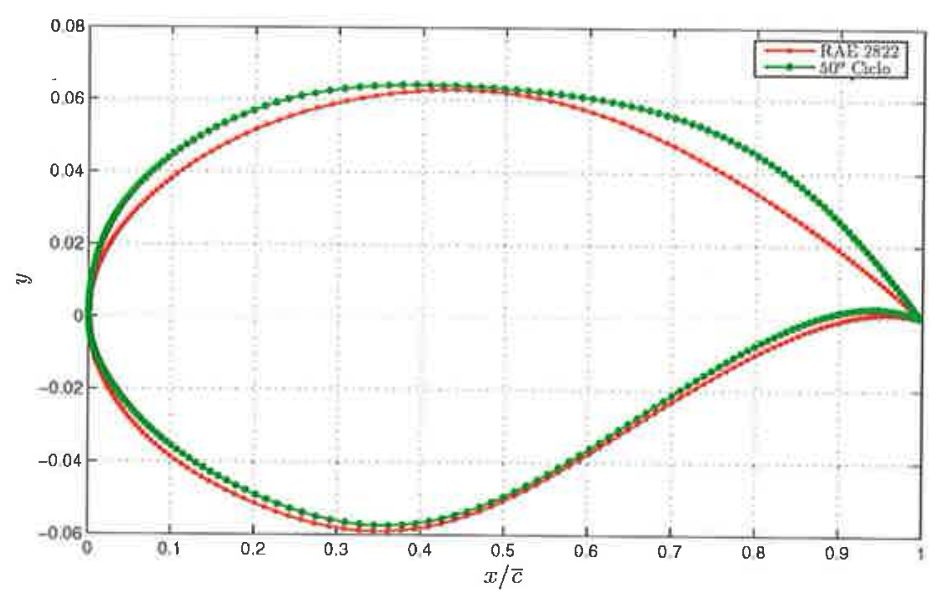

(b) Evolução da geometria

Figura 6.5: Caso de aplicação 1

responde à $I_{50}=0.0022$. Nota-se que a variação da medida de mérito é bem menor neste caso. Entretanto, vale lembrar que a edição da distribuição objetivo corresponde a pequenas variações. Além disso, o fato de propor uma distribuição objetivo que não necessariamente é realizável implica na impossibilidade de obter uma geometria final com medida de mérito nula.

\section{Caso de Aplicação 2}

Já a figura 6.6 apresenta outro caso de aplicação em que o perfil inicial é o mesmo do caso anterior, porém sujeito a um escoamento incidindo sobre o aerofólio com $1.0^{\circ}$. A distribuição desejada também foi editada visando a eliminação da onda de choque. E, assim como o caso anterior, apesar de não conseguir eliminar a onda de choque, minimizou-se a diferença entre a distribuição de pressão inicial e a desejada.

Neste caso, a medida de mérito da geometria inicial é de $I_{0}=0.0072$ e, 


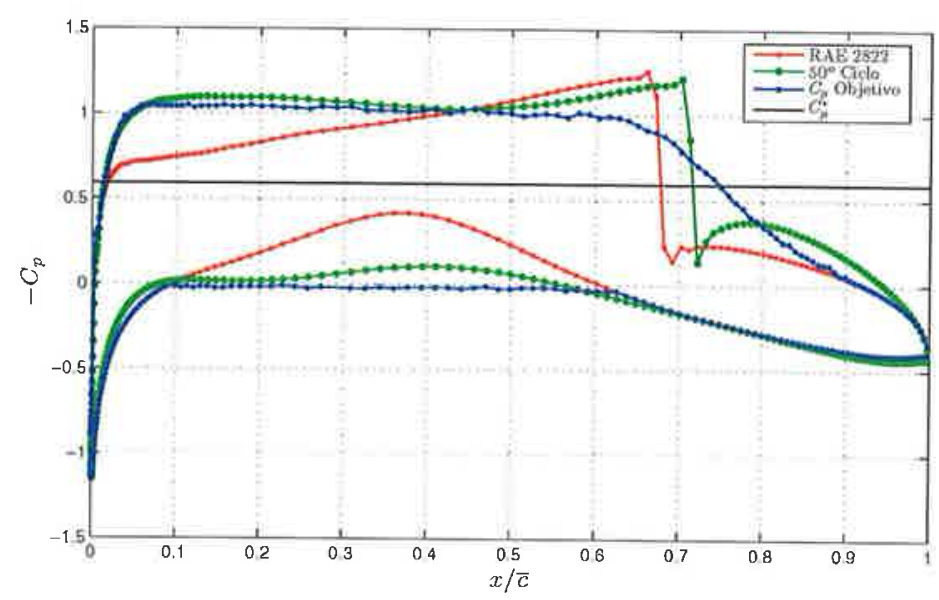

(a) Evolução de $C_{p}$

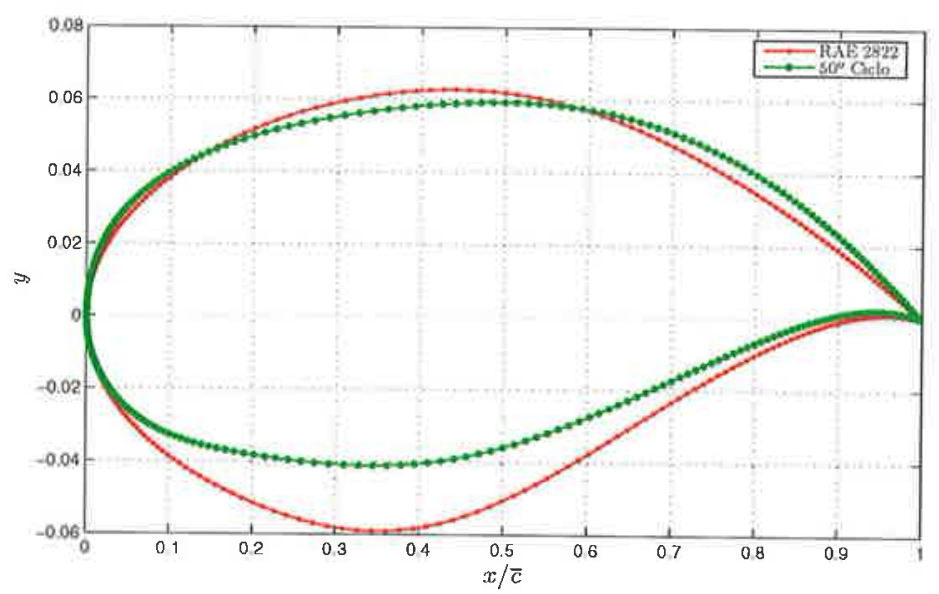

(b) Evolução da geometria

Figura 6.6: Caso de aplicação 2

após os 50 ciclos, obteve-se uma geometria tal que $I_{50}=0.0016$. Note, ainda, que a distribuição de pressões da geometria final ainda diminuiu o coeficiente de momento em relação a geometria inicial, o que ainda pode contribuir para uma redução do arrasto de trimagem.

\subsection{Sentido de Propagação das Perturbações}

Neste ponto, vale ilustrar o caráter hiperbólico das equações de Euler e adjuntas, que implica na existência de soluções na forma de onda. Ao analisar o transiente de uma solução do problema primal ou dual ${ }^{1}$, é possível identificar as perturbações, que são propagadas na velocidade das características e, assim, vizualizar o efeito da inclusão dos termos tempo-dependentes em ambos os problemas garantindo que eles sejam complementares.

\footnotetext{
${ }^{1}$ Neste caso é necessário utilizar um passo de tempo fixo ao realizar a marcha no tempo.
} 
Uma vez que a solução inicial das equações de Euler é um campo uniforme com as condições de escoamento não-perturbado, os resíduos da solução representam as perturbações que ocorrem no domínio computacional ao perceber a presença de uma superfície sólida (no caso, o aerofólio).

A figura 6.7 mostra os resíduos das equações de conservação de massa, quantidade de movimento e energia de uma solução intermediária não-convergida. Note que a propagação de perturbações ocorre em todas as direções, praticamente em forma de circunferência, que aumenta de diâmetro e tem seu centro deslocado para a direita a cada instante de tempo.

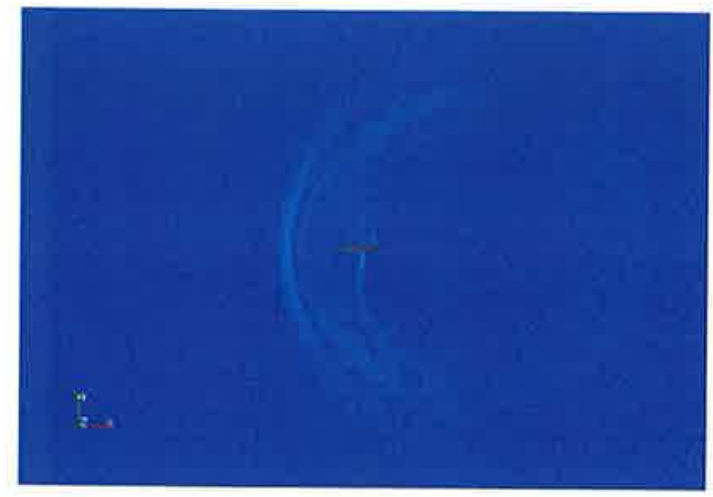

(a) Massa

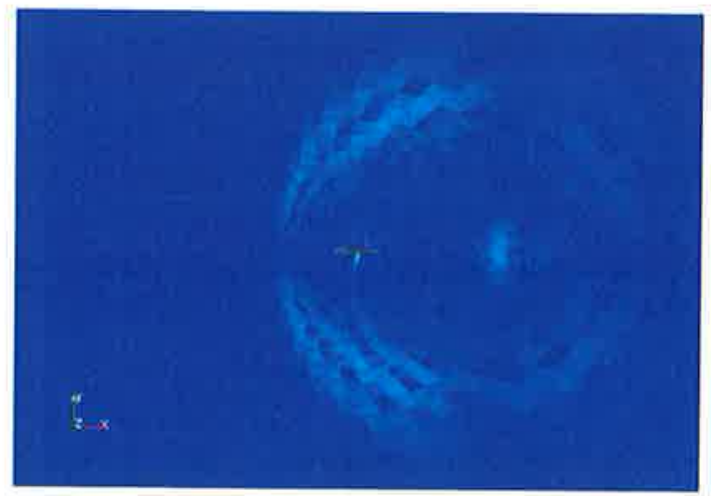

(c) Quantidade de movimento em y

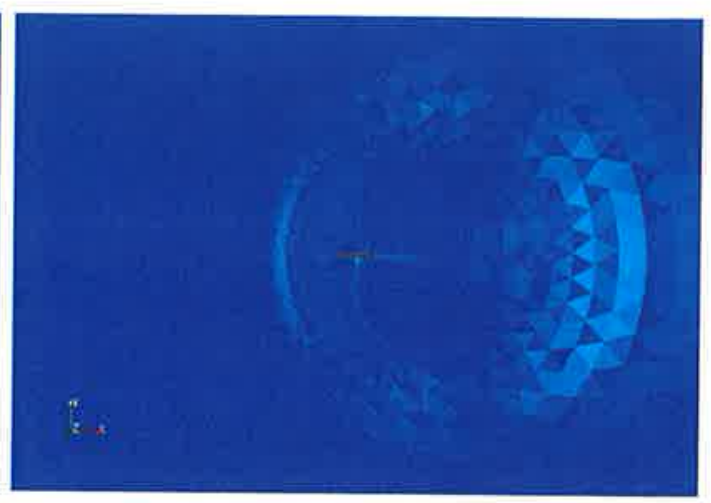

(b) Quantidade de movimento em $\mathrm{x}$

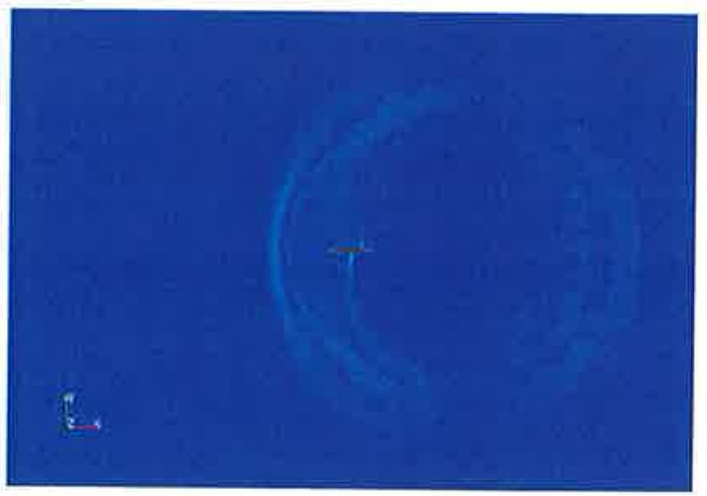

(d) Energia

Figura 6.7: Propagação de perturbações do escoamento

Já o código que resolve as equações adjuntas tem a solução homogênea como inicial. A propagação de perturbações ocorre devido à integral da medida de mérito não-nula, que nos casos simulados é, apenas, um termo da condição de contorno de parede. Assim, as perturbações da solução adjunta ocorrem de maneira similar às das equações de Euler, partido da superfície sólida em direção ao farfield. A figura 6.8 mostra os resíduos das equações que resolvem cada uma das 4 variáveis adjuntas durante uma solução intermediária, também, não-convergida.

A comparação entre as figuras 6.7 e 6.8 mostra, claramente, o efeito da inclusão dos termos tempo-dependentes nas equações de Euler e adjuntas. Natu- 
ralmente, como já dito no capítulo 3, postular estes termos de maneira diferente não altera a solução em estado estacionário de nenhum deles ${ }^{2}$, mas possibilita que suas características sejam complementares, já que o sentido propagação das perturbações dos dois problemas ocorre de maneira espelhada.

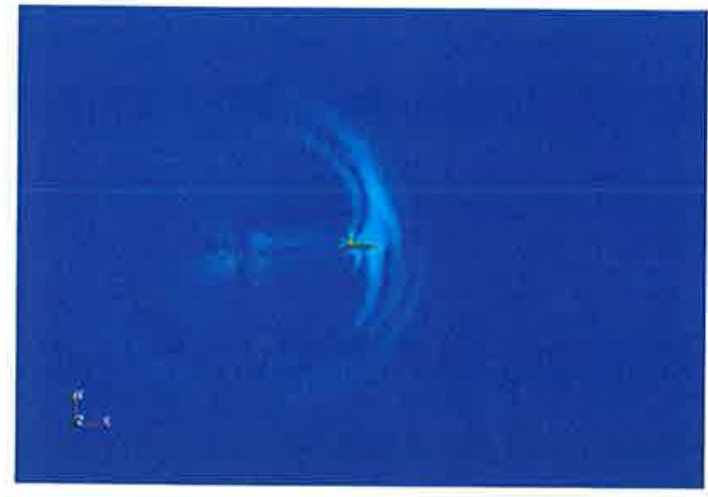

(a) $\psi_{1}$

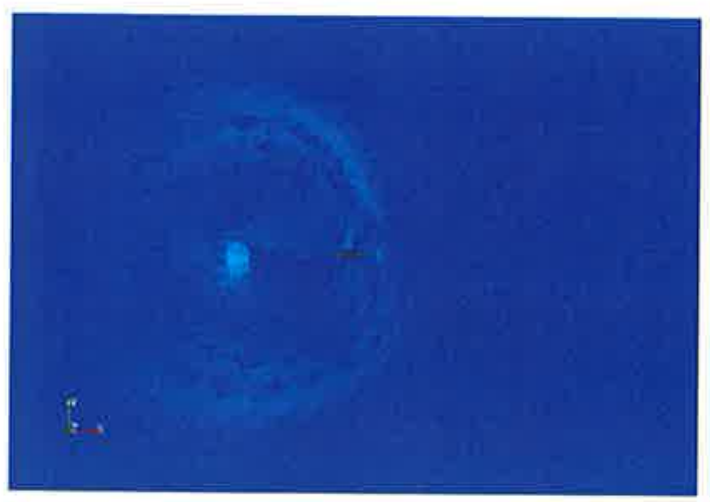

(c) $\psi_{3}$

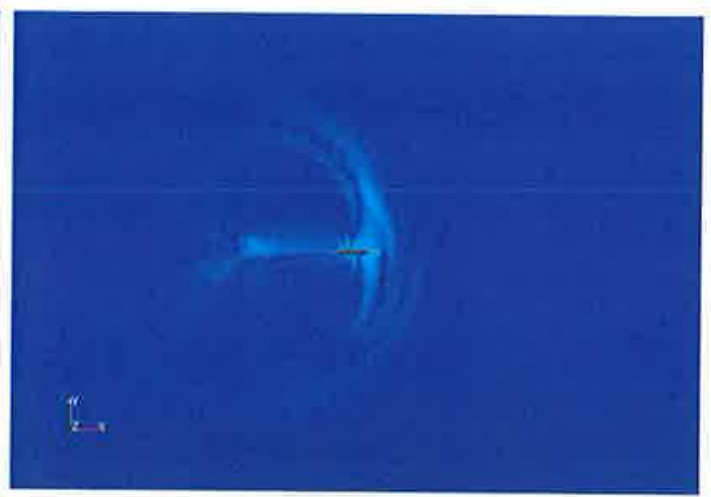

(b) $\psi_{2}$

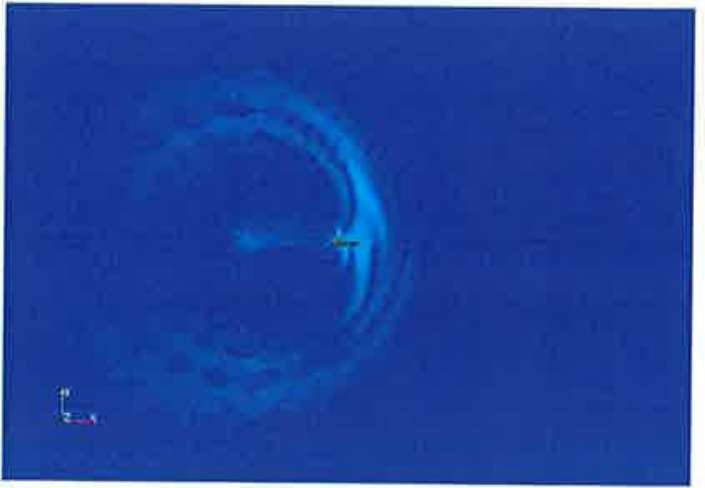

(d) $\psi_{4}$

Figura 6.8: Propagação de perturbações da solução adjunta

Isto torna possível todo o desenvolvimento de condições de contorno proposto neste trabalho, pois permite que os graus de liberdade livres do problema de contorno primal forneçam a informação necessária para "completar" o problema dual, uma vez que possibilita que sejam "fixadas" relações entre as variáveis adjuntas (à princípio uma tarefa impossível, já que as variáveis adjuntas não representam nenhuma propriedade física) à partir da equação que representa a condição de contorno de farfield.

\footnotetext{
${ }^{2}$ Vale lembrar que a proposta deste trabalho é a otimização de acrofólios em escoamentos nas condições de estado estacionário.
} 


\section{Conclusões e Trabalhos FUTURos}

Nesta dissertação desenvolveu-se uma rotina de otimização aerodinâmica baseada no método adjunto para escoamentos modelados pelas equações de Euler bidimensionais em malhas não-estruturadas de triângulos. Entretanto, o maior mérito do trabalho está na metodologia de obtenção das condições de contorno, em que se estendeu o procedimento proposto por Volpe e Santos (2009) para escoamentos em bocais quase-unidimensionais para um caso multidimensional.

\subsection{Conclusões}

A utilização da formulação Euler e não Navier-Stokes, como modelo físico do escoamento, deve se, basicamente, a dois motivos: primeiro, esta formulação é simples o bastante para os objetivos traçados e limitações de tempo e recursos; segundo, ela retém a não-linearidade dada pelo termo convectivo, que é absolutamente determinante para o problema de contorno.

O código de solução das equações que governam o escoamento foi desenvolvido com condições de contorno baseadas em equações diferenciais características, típicamente utilizadas para resolver escoamentos internos. Os resultados obtidos computacionalmente são condizentes com os obtidos em softwares já consagrados como o $\mathrm{CFD}++$, mostrando que, apesar de pouco utilizadas para escoamentos externos, devido ao seu alto custo computacional, as condições de contorno implementadas convergem para as recomendadas pela literatura (invariantes de Riemann).

Já o problema adjunto é formulado à imagem e semelhança das equações de Euler 2-D e suas condições de contorno são obtidas à partir de equações diferenciais características e relações de compatibilidade ao invés de impor as condições homogêneas citadas pela literatura. Desta forma, evita-se a sobrespecificação do problema (freqüente causa de não-unicidade da solução e, conseqüentemente, de 
um problema matematicamente mal-posto), garantindo que ele seja tão bemposto quanto o problema original.

Considera-se que o procedimento de formulação do problema de contorno proposto está validado, uma vez que resultados de casos similares aos apresentados por Ceze (2008), que utilizou o procedimento recomendado pela literatura, convergiram para as mesmas soluções. Além disso, este procedimento garante três objetivos principais:

- Compatibilidade entre os problemas primal e dual;

- Problema dual bem-posto;

- Eliminação do termo correspondente na variação do funcional aumentado.

Com relação aos resultados dos casos de validação e aplicação apresentados no capítulo 6 , é interessante observar a capacidade que o método adjunto tem de tratar ondas de choque quando formulado à partir das equações de Euler. Muitos dos métodos consagrados em uso na indústria são também, certamente, capazes de posicionar choques, entretanto, com maior dificuldade, já que a física capturada por estes métodos não necessariamente contempla esse tipo de fenômeno nãolinear.

Por fim, dentre as grandes vantagens do método adjunto com relação à outros métodos de otimização e projeto inverso em uso na indústria, destacam-se a sua enorme flexibilidade na definição de diferentes medidas de mérito, seu baixo custo computacional e a liberdade de escolha do modelo físico do escoamento, do escoamento potencial linearizado à Navier-Stokes.

\subsection{Trabalhos Futuros}

Os resultados obtidos neste trabalho trazem expectativas promissoras com relação às condições de contorno propostas. Uma vez utilizadas em escoamentos externos, pode-se pensar em otimizar superfícies aerodinâmicas em escoamentos internos. Afinal, às condições de contorno apresentadas devem ser apropriadas para estes casos em que as perturbações chegam às fronteiras com maior intensidade.

Em princípio, a rotina de otimização desenvolvida pode, ainda, servir de base para a continuação das pesquisas com o método adjunto. Pode-se utilizar este trabalho como ponto de partida para consolidar uma linha de pesquisas na área, e 
desenvolver futuros projetos de iniciação científica, mestrado e doutorado. Segue, abaixo, alguns possíveis tópicos de trabalhos futuros:

- Projeto multi-ponto;

- Utilização de outros métodos de otimização além do steepest descent;

- Implementação de outras medidas de mérito;

- Implementação da formulação bidimensional em aplicações tridimensionais, via seções de controle;

- Implementação das condições de contorno desenvolvidas para otimização de superfícies em escoamentos internos;

- Extensão desta rotina para uma formulação Euler tridimensional;

- Extensão desta rotina para uma formulação Navier-Stokes bidimensional;

- Em testes 3-D, pode-se analisar aplicações de interferência aerodinâmica.

Enfim, as diversas possibilidades de continuação deste trabalho abrem uma possibilidade de consolidar, de vez, uma linha de pesquisa nesta área no NDF que possa render frutos para indústria e a academia. 


\section{REFERÊNCIAS}

AZEVEDO, J. L. F. Notas de aula. Métodos Numéricos para Escoamento Compressível I. 2006.

AZEVEDO, J. L. F.; DOURADO, W. M. C. Euler solutions of two dimensional flows using unstructured meshes. In: Anais do XI Congresso Brasileiro de Engenharia Mecânica - XI COBEM. São Paulo: [s.n.], 1991. p. 189-192.

CEZE, M. A. B. Projeto Inverso Aerodinâmico Utilizando o Método Adjunto Aplicado às Equações de Euler. Dissertação (Mestrado) - Escola Politécnica da USP, 2008.

CHIEREGATTI, B. G. Otimização aerodinâmica de aerofólios utilizando o método adjunto. Trabalho de Graduação - EPUSP. 2008.

COURANT, R.; FRIEDRICHS, K. O.; LEWY, H. On the partial difference equations of mathematical physics. IBM Journal, v. 11, p. 215-234, 1967.

FLETCHER, C. A. J. Computational Techniques for Fluid Dynamics 1 Fundamental and General Techniques. 2nd. ed. [S.l.]: Springer-Verlag, 1990.

FLETCHER, C. A. J. Computational Techniques for Fluid Dynamics 2-Specific Techniques for Different Flow Categories. 2nd. ed. [S.l.]: Springer-Verlag, 1990.

FORTUNA, A. de O. Técnicas Computacionais para Dinâmica dos Fluidos: Conceitos Básicos e Aplicações. São Paulo: Edusp, 2000.

FUJITA, T. Efficient wing design for complex aircraft configuration using cad, unstructured cfd and inverse problems. In: ICAS. ICAS 2002 Congress. [S.1.], 2002. p. 231.1-231.9.

GANTMACHER, F. Lectures in Analytical Mechanics. [S.l.]: MIR Publishers Moscow, 1975.

GILES, M. B. Aerospace Design: a Complex Task. Oxford, July 1997.

GILES, M. B.; PIERCE, N. A. Adjoint Equations in CFD: Duality, Boundary Conditions and Solution Behavior. 1997. AIAA Paper 97-1850.

GILES, M. B.; PIERCE, N. A. Adjoint equations in cfd: duality, boundary conditions and solution behaviour. In: AIAA Paper 97-1850. [S.l.: s.n.], 1997.

GILES, M. B.; PIERCE, N. A. On the properties of the adjoint euler equations. In: BAINES, M. J. (Ed.). Numerical Methods for Fluid Dynamics VI. [S.l.: s.n.], 1998.

GILES, M. B.; PIERCE, N. A. Superconvergent lift estimates through adjoint error analysis. 1998. 
GILES, M. B.; PIERCE, N. A. Adjoint recovery of superconvergent functionals from approximate solutions of partial differential equations. Oxford, August 1999.

GILES, M. B.; PIERCE, N. A. Improved lift and drag estimates using adjoint Euler equations. 1999. AIAA.

GILES, M. B.; PIERCE, N. A. Analytic Adjoint Solutions for the Quasi-1D Euler Equations. Oxford, March 2000.

GILES, M. B.; PIERCE, N. A. An introduction to the adjoint approach to design. Flow, Turbulence and Combustion, v. 65, p. 393-415, 2000.

HADAMARD, J. Lectures on Cauchy's Problem in Linear Partial Differential Equations. New York: Dover Publications, Inc., 1952.

HICKS, R. M.; HENNE, P. A. Wing design by numerical optimization. Journal of Aircraft, v. 15, n. 7, p. 407-412, July 1978.

HICKS, R. M.; MURMAN, E. M.; VANDERPLAATS, G. N. An Assessment of Airfoil Design by Numerical Optimization. Moffett Field, CA, July 1974. NASA TM X-3092.

HIRSCH, C. Numerical Computation of Internal and External Flows. Vol. 1: Fundamentals of Numerical Discretization. [S.l.]: John Wiley \& Sons, 1988.

HIRSCH, C. Numerical Computation of Internal and External Flows. Vol. 2: Computational Methods for Inviscid and Viscous Flows. [S.l.]: John Wiley \& Sons, 1988.

HIRSCH, C. Numerical Computation of Internal and External Flows. Vol. 1: The Fundamentals of Computational Fluid Dynamics. 2nd. ed. [S.1.]: John Wiley \& Sons Ltd., 2007.

JAMESON, A. Aerodynamic design via control theory. In: 12th IMACS World Congress on Scientific Computation. Paris: [s.n.], 1988. (MAE Report 1824).

JAMESON, A. Airfoils admitting non--unique solutions of the euler equations. In: AIAA 22nd Fluid Dynamics, Plasmadynamics and Lasers Conference. Honolulu, HI: [s.n.], 1991.

JAMESON, A. Optimum Aerodynamic Design via Boundary Control. [S.l.], April 1994.

JAMESON, A. Re-engineering the design process through computation. In: 35th Aerospace Sciences Meeting \& Exhibit. Reno, NV: AIAA, 1997. AIAA-97-0641.

JAMESON, A. Aerodynamic Shape Optimization Using the Adjoint Method. February 2003. Lectures at the Von Kármán Institute, Brussels.

JAMESON, A.; KIM, S. Reduction of the adjoint gradient formula for aerodynamic shape optimization problems. AIAA Journal, v. 41, n. 11, p. 2114-2129, November 2003.

JAMESON, A.; KIM, S. Reduction of the adjoint gradient formula in the continuous limit. In: AIAA Computational Fluid Dynamics Conference. [S.1.: s.n.], 2003. AIAA 2003-0040. 
JAMESON, A.; MAVRIPLIS, D. Finite volume solution of the two-dimensional euler equations on a regular triangular mesh. AIAA Journal, v. 24, n. 4, p. 611-618, April 1986.

JAMESON, A.; NADARAJAH, S. K. A comparison of the continuous and discrete adjoint approach to automatic aerodynamic optimization. In: AIAA 38th Aerospace Sciences Meeting and Exhibit. Reno, NV: [s.n.], 2000.

JAMESON, A.; SCHIMIDT, W.; TURKEL, E. Numerical solution of the euler equations by finite volume methods using runge-kutta time-stepping schemes. AIAA Journal, 1981.

JAMESON, A.; SRIRAM; MARTINELLI, L. A continuous adjoint method for unstructured grids. In: AIAA. 16th CFD Conference. Orlando, FL, 2003.

KIM, H. J.; KOC, S.; NAKAHASHI, K. Aerodynamic design of wingbody-nacelle-pylon configuration. In: AIAA Computational Fluid Dynamics Conference. [S.l.: s.n.], 2005.

KULFAN, B. M.; BUSSOLETTI, J. E. Fundamental parametric geometry representations for aircraft component shapes. In: 11th AIAA/ISSMO Multidisciplinary Analysis and Optimization Conference. Portsmouth, VA: [s.n.], 2006.

LANEY, G. B. Computational Gasdynamics. New York, NY: Cambridge University Press, 1998.

LEVEQUE, R. J. Finite-Volume Methods for Hyperbolic Problems. [S.l.]: Cambridge University Press, 2002. (Cambridge Texts in Applied Mathematics).

LIONS, J. L. Optimal Control of Systems Governed by Partial Differential Equations. 1st. ed. Berlin: Spirnger-Verlag, 1971. (Die Grundelehren der mathematischen Wissenschaften, 170).

LOMAX, H.; PULLIAM, T. H.; ZINGG, D. W. Fundamentals of Computational Fluid Dynamics. [S.l.]: Springer-Verlag Berlin, 2001.

MACCORMACK, R. A perspective on a quarter century of cfd research. In: 11th AIAA Computational Fluid Dynamics Conference. Orlando, FL: [s.n.], 1993. AIAA-93-3291.

MAKINO, Y.; IWAMIYA, T.; LEI, Z. Fuselage shape optimization of a wing-body configuration with nacelles. Journal of Aircraft, v. 40, n. 2, p. 297-302, March-April 2003.

MALISKA, C. R. Transferência de Calor e Mecânica dos Fluidos Computacional. $2^{\mathrm{a}}$. ed. Rio de Janeiro, RJ: LTC, 2004.

NAIK, D. A.; KRIST, S. E.; CAMPBELL, R. L.; VATSA, V. N.; BUNING, P. G.; GEA, L. M. Inverse design of nacelles using multi-block Navier-Stokes codes. In: AMERICAN INSTITUTE OF AERONAUTICS AND ASTRONAUTICS. AIAA 33st Aerospace Sciences Meeting and Exhibit. Reno, NV: AIAA, 1995. AIAA 1995-1820. 
OLIVEIRA, G. L.; TRAPP, L. G.; MACEDO, A. P. Engine-airframe integration methodologies for regional jet aircraft with underwing engines. In: AMERICAN INSTITUTE OF AERONAUTICS AND ASTRONAUTICS. AIAA 41st Aerospace Sciences Meeting and Exhibit. Reno, NV: AIAA, 2003. AIAA 2003-0934.

PIRONNEAU, O. Optimal Shape Design for Elliptic Systems. New York: Springer-Verlag, 1983. (Springer Series in Computational Physics).

PRAGER, W. Introduction to Mechanics of Continua. [S.l.]: Ginn and Company, 1961.

PULLIAM, T. H. Solution methods in computational fluid dynamics. Lecture Notes for the Von Kármán Institute for Fluid Dynamics. 1985.

REUTHER, J. J. Aerodynamic Shape Optimization Using Control Theory. Tese (Doutorado) - University of California Davis, 1996.

RODRIGUEZ, D. Response surface based optimization with a cartesian cfd method. In: AMERICAN INSTITUTE OF AERONAUTICS AND ASTRONAUTICS. AIAA 41st Aerospace Sciences Meeting and Exhibit. Reno, NV: AIAA, 2003. AIAA 2003-0465.

SADRI, R.; LEBLOND, D.; PIPERNI, P. Coupling of a wing inverse design code to an euler/navier-stokes flow solver using mesh movement capabilities. Advanced Aerodynamics, Bombardier Aerospace. 2002.

SANTOS, L. C. C. A Hybrid Inverse Optimization Method for Aerodynamic Design of Lifting Surfaces. Tese (Doutorado) - Georgia Institute of Technology, 1993.

SANTOS, L. C. C. A Study on Aerodynamic Design Optimization Using An Adjoint Method. Braunschweig, July 1995.

SWANSON, R. C.; RADESPIEL, R. Cell centered and cell vertex multigrid schemes for the navier-stokes equations. AIAA Journal, v. 29, n. 5, p. 697-703, May 1991.

VOLPE, E. V. Notas de aula. Álgebra Tensorial Generalizada. 1993.

VOLPE, E. V. A11 - Inverse Aerodynamic Design Module. [S.1.], 2004.

VOLPE, E. V. A3 - Inverse Aerodynamic Design Applications. [S.1.], 2005.

VOLPE, E. V.; OLIVEIRA, G. L.; SANTOS, L. C. C.; HAYASHI, M. T.; CEZE, M. A. B. Inverse aerodynamic design applications usign the mgm hybrid formulation. In: Inverse Problems Design and Optimization Symposium. Miami, FL: [s.n.], 2007.

VOLPE, E. V.; SANTOS, L. C. C. Boundary and internal conditions for adjoint fluid flow problems - applications to quasi-1d euler equations. Journal of Engineering Mathematics (paper accepted for publication). 2009. 


\section{ApÊNDICE A - As EquAÇões de Euler em CoORdenadas Generalizadas}

Considere as equações de Euler bidimensionais em sua forma diferencial conservativa escritas em coordenadas cartesianas:

$$
\frac{\partial \mathbf{Q}}{\partial t}+\frac{\partial \mathbf{E}}{\partial x}+\frac{\partial \mathbf{F}}{\partial y}=0
$$

onde o vetor de variáveis conservadas $\mathbf{Q}$ e os vetores de fluxo $\mathbf{E}$ e $\mathbf{F}$ são representados por:

$$
\mathbf{Q}=\left(\begin{array}{c}
\rho \\
\rho u \\
\rho v \\
e
\end{array}\right) \quad, \quad \mathbf{E}=\left(\begin{array}{c}
\rho u \\
\rho u^{2}+p \\
\rho u v \\
(e+p) u
\end{array}\right) \quad, \quad \mathbf{F}=\left(\begin{array}{c}
\rho v \\
\rho u v \\
\rho v^{2}+p \\
(e+p) v
\end{array}\right)
$$

Assumindo uma transformação de coordenadas que transforma o espaço cartesiano no espaço transformado ao redor de um aerofólio indicada na figura A.1 e dada por:

$$
\begin{aligned}
\tau & =t \\
\xi & =\xi(x, y, t) \\
\eta & =\eta(x, y, t)
\end{aligned}
$$
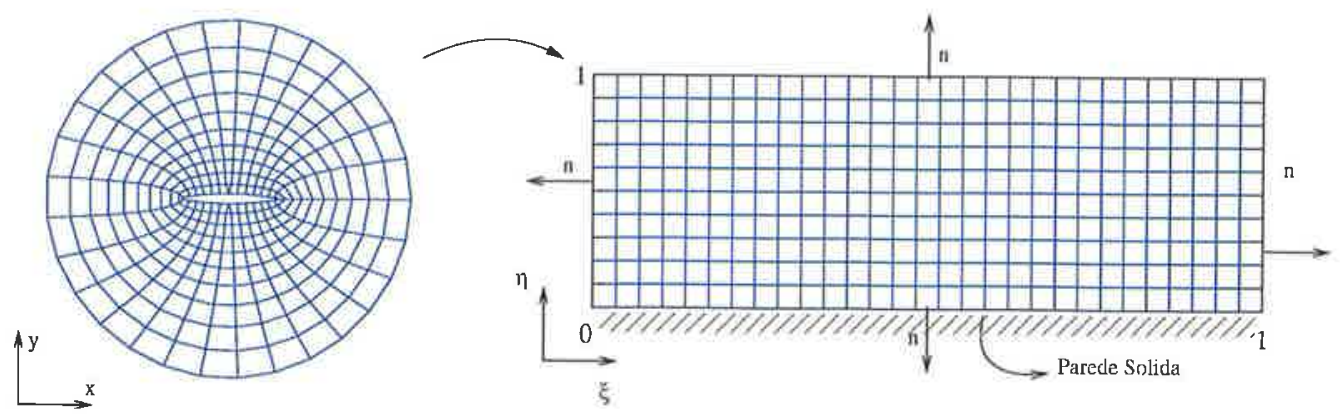

Figura A.1: Transformação de coordenadas 
Então, utilizando a regra da cadeia, pode-se escrever:

$$
\begin{aligned}
& \frac{\partial \mathbf{Q}}{\partial t}=\frac{\partial \mathbf{Q}}{\partial \tau} \frac{\partial f^{1}}{\partial t}+\frac{\partial \mathbf{Q}}{\partial \xi} \frac{\partial \xi}{\partial t}+\frac{\partial \mathbf{Q}}{\partial \eta} \frac{\partial \eta}{\partial t}=\frac{\partial \mathbf{Q}}{\partial \tau}+\xi_{t} \frac{\partial \mathbf{Q}}{\partial \xi}+\eta_{t} \frac{\partial \mathbf{Q}}{\partial \eta} \\
& \frac{\partial \mathbf{E}}{\partial x}=\frac{\partial \mathbf{E}}{\partial \tau} \frac{\partial f^{\prime}}{\partial x}+\frac{\partial \mathbf{E}}{\partial \xi} \frac{\partial \xi}{\partial x}+\frac{\partial \mathbf{E}}{\partial \eta} \frac{\partial \eta}{\partial x}=\xi_{x} \frac{\partial \mathbf{E}}{\partial \xi}+\eta_{x} \frac{\partial \mathbf{E}}{\partial \eta} \\
& \frac{\partial \mathbf{F}}{\partial y}=\frac{\partial \mathbf{F}}{\partial \tau} \frac{\partial f^{\prime}}{\partial y}+\frac{\partial \mathbf{F}}{\partial \xi} \frac{\partial \xi}{\partial y}+\frac{\partial \mathbf{F}}{\partial \eta} \frac{\partial \eta}{\partial y}=\xi_{y} \frac{\partial \mathbf{F}}{\partial \xi}+\eta_{y} \frac{\partial \mathbf{F}}{\partial \eta}
\end{aligned}
$$

Substituindo (A.3), (A.4) e (A.5) em (A.1), obtém-se:

$$
\frac{\partial \mathbf{Q}}{\partial \tau}+\xi_{t} \frac{\partial \mathbf{Q}}{\partial \xi}+\eta_{t} \frac{\partial \mathbf{Q}}{\partial \eta}+\xi_{x} \frac{\partial \mathbf{E}}{\partial \xi}+\eta_{x} \frac{\partial \mathbf{E}}{\partial \eta}+\xi_{y} \frac{\partial \mathbf{F}}{\partial \xi}+\eta_{y} \frac{\partial \mathbf{F}}{\partial \eta}=0
$$

Multiplicando (A.6) pelo Jacobiano da transformação $J$, tem-se:

$$
\begin{gathered}
\underbrace{J \frac{\partial \mathbf{Q}}{\partial \tau}}_{I}+\underbrace{J \xi_{t} \frac{\partial \mathbf{Q}}{\partial \xi}}_{I I}+\underbrace{J \eta_{t} \frac{\partial \mathbf{Q}}{\partial \eta}}_{I I I}+\underbrace{J \xi_{x} \frac{\partial \mathbf{E}}{\partial \xi}}_{I V}+\underbrace{J \eta_{x} \frac{\partial \mathbf{E}}{\partial \eta}}_{V}+ \\
\underbrace{J \xi_{y} \frac{\partial \mathbf{F}}{\partial \xi}}_{V I}+\underbrace{J \eta_{y} \frac{\partial \mathbf{F}}{\partial \eta}}_{V I I}=0
\end{gathered}
$$

onde,

$$
J=\left|\frac{\partial(t, x, y)}{\partial(\tau, \xi, \eta)}\right|=\left|\begin{array}{ccc}
1 & x_{\tau} & y_{\tau} \\
0 & x_{\xi} & y_{\xi} \\
0 & x_{\eta} & y_{\eta}
\end{array}\right|=x_{\xi} y_{\eta}-x_{\eta} y_{\xi}
$$

Mas, pela regra da cadeia:

$$
\begin{aligned}
I & \rightarrow \frac{\partial}{\partial \tau}(J \mathbf{Q})=\mathbf{Q} \frac{\partial}{\partial \tau}(J)+J \frac{\partial \mathbf{Q}}{\partial \tau} \\
I I & \rightarrow \frac{\partial}{\partial \xi}\left(J \xi_{t} \mathbf{Q}\right)=\mathbf{Q} \frac{\partial}{\partial \xi}\left(J \xi_{t}\right)+J \xi_{t} \frac{\partial \mathbf{Q}}{\partial \xi} \\
I I I & \rightarrow \frac{\partial}{\partial \eta}\left(J \eta_{t} \mathbf{Q}\right)=\mathbf{Q} \frac{\partial}{\partial \eta}\left(J \eta_{t}\right)+J \eta_{t} \frac{\partial \mathbf{Q}}{\partial \eta} \\
I V & \rightarrow \frac{\partial}{\partial \xi}\left(J \xi_{x} \mathbf{E}\right)=\mathbf{E} \frac{\partial}{\partial \xi}\left(J \xi_{x}\right)+J \xi_{x} \frac{\partial \mathbf{E}}{\partial \xi} \\
V & \rightarrow \frac{\partial}{\partial \eta}\left(J \eta_{x} \mathbf{E}\right)=\mathbf{E} \frac{\partial}{\partial \eta}\left(J \eta_{x}\right)+J \eta_{x} \frac{\partial \mathbf{E}}{\partial \eta} \\
V I & \rightarrow \frac{\partial}{\partial \xi}\left(J \xi_{y} \mathbf{F}\right)=\mathbf{F} \frac{\partial}{\partial \xi}\left(J \xi_{y}\right)+J \xi_{y} \frac{\partial \mathbf{F}}{\partial \xi} \\
V I I & \rightarrow \frac{\partial}{\partial \eta}\left(J \eta_{y} \mathbf{F}\right)=\mathbf{F} \frac{\partial}{\partial \eta}\left(J \eta_{y}\right)+J \eta_{y} \frac{\partial \mathbf{F}}{\partial \eta}
\end{aligned}
$$


Substituindo (A.9)-(A.15) em (A.7), chega-se a seguinte expressão:

$$
\begin{array}{r}
\frac{\partial}{\partial \tau}(J \mathbf{Q})-\mathbf{Q} \frac{\partial}{\partial \tau}(J)+\frac{\partial}{\partial \xi}\left(J \xi_{t} \mathbf{Q}\right)-\mathbf{Q} \frac{\partial}{\partial \xi}\left(J \xi_{t}\right)+\frac{\partial}{\partial \eta}\left(J \eta_{t} \mathbf{Q}\right)+ \\
-\mathbf{Q} \frac{\partial}{\partial \eta}\left(J \eta_{t}\right)+\frac{\partial}{\partial \xi}\left(J \xi_{x} \mathbf{E}\right)-\mathbf{E} \frac{\partial}{\partial \xi}\left(J \xi_{x}\right)+\frac{\partial}{\partial \eta}\left(J \eta_{x} \mathbf{E}\right)+ \\
-\mathbf{E} \frac{\partial}{\partial \eta}\left(J \eta_{x}\right)+\frac{\partial}{\partial \xi}\left(J \xi_{y} \mathbf{F}\right)-\mathbf{F} \frac{\partial}{\partial \xi}\left(J \xi_{y}\right)+\frac{\partial}{\partial \eta}\left(J \eta_{y} \mathbf{F}\right)+ \\
-\mathbf{F} \frac{\partial}{\partial \eta}\left(J \eta_{y}\right)=0
\end{array}
$$

Rearranjando os termos de forma conveniente, obtém-se:

$$
\begin{array}{r}
\frac{\partial}{\partial \tau}(J \mathbf{Q})+\frac{\partial}{\partial \xi}\left[J\left(\xi_{t} \mathbf{Q}+\xi_{x} \mathbf{E}+\xi_{y} \mathbf{F}\right)\right]+\frac{\partial}{\partial \eta}\left[J\left(\eta_{t} \mathbf{Q}+\eta_{x} \mathbf{E}+\eta_{y} \mathbf{F}\right)\right]+ \\
-\left\{\mathbf{Q}\left[\frac{\partial}{\partial \tau}(J)+\frac{\partial}{\partial \xi}\left(J \xi_{t}\right)+\frac{\partial}{\partial \eta}\left(J \eta_{t}\right)\right]+\right. \\
+\mathbf{E}\left[\frac{\partial}{\partial \xi}\left(J \xi_{x}\right)+\frac{\partial}{\partial \eta}\left(J \eta_{x}\right)\right]+ \\
\left.+\mathbf{F}\left[\frac{\partial}{\partial \xi}\left(J \xi_{y}\right)+\frac{\partial}{\partial \eta}\left(J \eta_{y}\right)\right]\right\}=0
\end{array}
$$

As derivadas dos termos em coordenadas cartesianas podem ser expressas em função das derivadas dos termos em coordenadas generalizadas da seguinte maneira:

$$
\underbrace{\left\{\begin{array}{c}
\frac{\partial}{\partial t} \\
\frac{\partial}{\partial x} \\
\frac{\partial}{\partial y}
\end{array}\right\}}_{\left[D_{c}\right]}=\underbrace{\left[\begin{array}{ccc}
\frac{\partial f^{1}}{\partial t} & \frac{\partial \xi}{\partial t} & \frac{\partial \eta}{\partial t} \\
\frac{\partial f^{t}}{\partial x} & \frac{\partial \xi}{\partial x} & \frac{\partial \eta}{\partial x} \\
\frac{\partial f^{0}}{\partial y} & \frac{\partial \xi}{\partial y} & \frac{\partial \eta}{\partial y}
\end{array}\right]}_{[A]} \underbrace{\left\{\begin{array}{c}
\frac{\partial}{\partial \tau} \\
\frac{\partial}{\partial \xi} \\
\frac{\partial}{\partial \eta}
\end{array}\right\}}_{D_{g}} \Rightarrow\left\{D_{c}\right\}=\left[\begin{array}{ccc}
1 & \xi_{t} & \eta_{t} \\
0 & \xi_{x} & \eta_{x} \\
0 & \xi_{y} & \eta_{y}
\end{array}\right]\left\{D_{g}\right\}
$$

ou, de forma inversa:

$$
\left\{\begin{array}{c}
\frac{\partial}{\partial \tau} \\
\frac{\partial}{\partial \xi} \\
\frac{\partial}{\partial \eta}
\end{array}\right\}=\underbrace{\left[\begin{array}{ccc}
\frac{\partial x^{1}}{\partial \tau} & \frac{\partial x}{\partial \tau} & \frac{\partial y}{\partial \tau} \\
\frac{\partial f^{0}}{\partial \xi} & \frac{\partial x}{\partial \xi} & \frac{\partial y}{\partial \xi} \\
\frac{\partial f^{0}}{\partial \eta} & \frac{\partial x}{\partial \eta} & \frac{\partial y}{\partial \eta}
\end{array}\right]}_{[B]}\left\{\begin{array}{c}
\frac{\partial}{\partial t} \\
\frac{\partial}{\partial x} \\
\frac{\partial}{\partial y}
\end{array}\right\} \Rightarrow\left\{D_{g}\right\}=\left[\begin{array}{ccc}
1 & x_{\tau} & y_{\tau} \\
0 & x_{\xi} & y_{\xi} \\
0 & x_{\eta} & y_{\eta}
\end{array}\right]\left\{D_{c}\right\}
$$

Substituindo (A.19) em (A.18), obtém-se a relação:

$$
\left\{D_{c}\right\}=[A][B]\left\{D_{c}\right\} \Rightarrow[A][B]=[I]
$$

onde $[I]$ é a matriz identidade. 
Assim, pode-se afirmar que:

$$
[A]=[B]^{-1}
$$

Calculando-se, agora, $[X]=[B]^{-1}$, tem-se:

$$
[X][B]=[I]
$$

ou, ainda:

$$
\left[\begin{array}{lll}
X_{11} & X_{12} & X_{13} \\
X_{21} & X_{22} & X_{23} \\
X_{31} & X_{32} & X_{33}
\end{array}\right]\left[\begin{array}{lll}
1 & x_{\tau} & y_{\tau} \\
0 & x_{\xi} & y_{\xi} \\
0 & x_{\eta} & y_{\eta}
\end{array}\right]=\left[\begin{array}{lll}
1 & 0 & 0 \\
0 & 1 & 0 \\
0 & 0 & 1
\end{array}\right]
$$

Resolvendo o sistema, chega-se à seguinte matriz $[X]$

$$
[X]=\left[\begin{array}{ccc}
1 & J^{-1}\left(x_{\eta} y_{\tau}-x_{\tau} y_{\eta}\right) & J^{-1}\left(x_{\tau} y_{\xi}-x_{\xi} y_{\tau}\right) \\
0 & J^{-1} y_{\eta} & -J^{-1} y_{\xi} \\
0 & -J^{-1} x_{\eta} & J^{-1} x_{\xi}
\end{array}\right]
$$

Comparando as matrizes $[X]$ e $[A]$, chega-se as seguintes relações:

$$
\begin{aligned}
\xi_{t} & =-x_{\tau} \xi_{x}-y_{\tau} \xi_{y} \\
\xi_{x} & =J^{-1} y_{\eta} \\
\xi_{y} & =-J^{-1} x_{\eta} \\
\eta_{t} & =-x_{\tau} \eta_{x}-y_{\tau} \eta_{y} \\
\eta_{x} & =-J^{-1} y_{\xi} \\
\eta_{y} & =J^{-1} x_{\xi}
\end{aligned}
$$

Substituindo as relações obtidas no termo entre chaves $(\{\cdots\})$ da equação (A.17), obtém-se o seguinte resultado:

$$
\begin{array}{r}
\mathbf{Q}\left[\frac{\partial}{\partial \tau}(J)+\frac{\partial}{\partial \xi}\left(J \xi_{t}\right)+\frac{\partial}{\partial \eta}\left(J \eta_{t}\right)\right]+ \\
+\mathbf{E}\left[\frac{\partial}{\partial \xi}\left(J \xi_{x}\right)+\frac{\partial}{\partial \eta}\left(J \eta_{x}\right)\right]+ \\
+\mathbf{F}\left[\frac{\partial}{\partial \xi}\left(J \xi_{y}\right)+\frac{\partial}{\partial \eta}\left(J \eta_{y}\right)\right]=0
\end{array}
$$

Portanto, a equação (A.17) fica simplificada:

$$
\frac{\partial}{\partial \tau}(J \mathbf{Q})+\frac{\partial}{\partial \xi}\left[J\left(\xi_{t} \mathbf{Q}+\xi_{x} \mathbf{E}+\xi_{y} \mathbf{F}\right)\right]+\frac{\partial}{\partial \eta}\left[J\left(\eta_{t} \mathbf{Q}+\eta_{x} \mathbf{E}+\eta_{y} \mathbf{F}\right)\right]=0
$$


que ainda pode ser excrita como:

$$
\frac{\partial \overline{\mathbf{Q}}}{\partial \tau}+\frac{\partial \overline{\mathbf{E}}}{\partial \xi}+\frac{\partial \overline{\mathbf{F}}}{\partial \eta}=0
$$

onde o novo vetor de estados transformado $\overline{\mathrm{Q}}$ pode ser representado da seguinte forma:

$$
\overline{\mathbf{Q}}=J \mathbf{Q} \Rightarrow \overline{\mathbf{Q}}=J\left\{\begin{array}{c}
\rho \\
\rho u \\
\rho v \\
e
\end{array}\right\}
$$

E os vetores de fluxo transformados $\overline{\mathbf{E}}$ e $\overline{\mathbf{F}}$ :

$$
\begin{aligned}
& \overline{\mathbf{E}}=J\left(\xi_{t} \mathbf{Q}+\xi_{x} \mathbf{E}+\xi_{y} \mathbf{F}\right)=J\left\{\begin{array}{c}
\xi_{t} \rho+\xi_{x}(\rho u)+\xi_{y}(\rho v) \\
\xi_{t}(\rho u)+\xi_{x}\left(\rho u^{2}+p\right)+\xi_{y}(\rho u v) \\
\xi_{t}(\rho v)+\xi_{x}(\rho u v)+\xi_{y}\left(\rho v^{2}+p\right) \\
\xi_{t} e+\xi_{x}[(e+p) u]+\xi_{y}[(e+p) v]
\end{array}\right\} \\
& \overline{\mathbf{F}}=J\left(\eta_{t} \mathbf{Q}+\eta_{x} \mathbf{E}+\eta_{y} \mathbf{F}\right)=J\left\{\begin{array}{c}
\eta_{t} \rho+\eta_{x}(\rho u)+\eta_{y}(\rho v) \\
\eta_{t}(\rho u)+\eta_{x}\left(\rho u^{2}+p\right)+\eta_{y}(\rho u v) \\
\eta_{t}(\rho v)+\eta_{x}(\rho u v)+\eta_{y}\left(\rho v^{2}+p\right) \\
\eta_{t} e+\eta_{x}[(e+p) u]+\eta_{y}[(e+p) v]
\end{array}\right\}
\end{aligned}
$$

Definindo as componentes contravariantes de velocidade como:

$$
\begin{aligned}
& U=\xi_{t}^{0}+\xi_{x} u+\xi_{y} v \\
& V=\eta_{t}^{*}+\eta_{x} u+\eta_{y} v
\end{aligned}
$$

Pode-se escrever os vetores $\overline{\mathbf{E}}$ e $\overline{\mathbf{F}}$ da seguinte maneira:

$$
\overline{\mathbf{E}}=J\left\{\begin{array}{c}
\rho U \\
\rho u U+p \xi_{x} \\
\rho v U+p \xi_{y} \\
(e+p) U
\end{array}\right\} \quad, \quad \overline{\mathbf{F}}=J\left\{\begin{array}{c}
\rho V \\
\rho u V+p \eta_{x} \\
\rho v V+p \eta_{y} \\
(e+p) V
\end{array}\right\}
$$

ou, de outro modo:

$$
\begin{aligned}
& \overline{\mathbf{Q}} \equiv J \mathbf{Q} \\
& \overline{\mathbf{E}} \equiv \mathbf{E}\left(J \frac{\partial \xi}{\partial x}\right)+\mathbf{F}\left(J \frac{\partial \xi}{\partial y}\right) \\
& \overline{\mathbf{F}} \equiv \mathbf{E}\left(J \frac{\partial \eta}{\partial x}\right)+\mathbf{F}\left(J \frac{\partial \eta}{\partial y}\right)
\end{aligned}
$$


Assim, pode-se definir as matrizes Jacobianas $\mathbf{C}_{1}$ e $\mathbf{C}_{2}$ do sistema transformado de forma análoga às matrizes $\mathrm{A}$ e $\mathrm{B}$ do sistema cartesiano:

$$
\begin{aligned}
& \mathbf{C}_{1}=\frac{\partial \overline{\mathbf{E}}}{\partial \mathbf{Q}}=\frac{\partial \mathbf{E}}{\partial \mathbf{Q}}\left(J \frac{\partial \xi}{\partial x}\right)+\frac{\partial \mathbf{F}}{\partial \mathbf{Q}}\left(J \frac{\partial \xi}{\partial y}\right) \\
& \mathbf{C}_{2}=\frac{\partial \overline{\mathbf{F}}}{\partial \mathbf{Q}}=\frac{\partial \mathbf{E}}{\partial \mathbf{Q}}\left(J \frac{\partial \eta}{\partial x}\right)+\frac{\partial \mathbf{F}}{\partial \mathbf{Q}}\left(J \frac{\partial \eta}{\partial y}\right)
\end{aligned}
$$




\section{APÊndice B - UMA Propriedade dAS EQUAÇÕES DE EULER EM COORDENADAS GENERALIZADAS}

Nesta seção, será apresentada a demonstração da expressão (VOLPE, 1993):

$$
\delta S_{j^{\prime}}^{i} \frac{\partial \mathcal{F}_{\alpha}^{j^{\prime}}}{\partial \xi^{i}}=-\frac{\partial}{\partial \xi^{r}}\left(S_{j^{\prime}}^{r} \frac{\partial \mathcal{F}_{\alpha}^{j^{\prime}}}{\partial \xi^{i}} \delta \xi^{i}\right)
$$

Para isso, considere a seguinte equação:

$$
\frac{\partial S_{j^{\prime}}^{i}}{\partial \xi^{i}}=\frac{\partial}{\partial \xi^{i}}\left(J \beta_{j^{\prime}}^{i}\right)=0
$$

Então, pela regra da cadeia:

$$
\begin{aligned}
S_{j^{\prime}}^{i} \frac{\partial \mathcal{F}^{j^{\prime}}}{\partial \xi^{i}} & =\frac{\partial\left(S_{j^{\prime}}^{i} \mathcal{F}^{j^{\prime}}\right)}{\partial \xi^{i}}=\frac{1}{2} \frac{\partial}{\partial \xi^{i}}\left[\epsilon_{j^{\prime} p^{\prime} q^{\prime}} \epsilon^{i r s}\left(\frac{\partial x^{p}}{\partial \xi^{r}} \frac{\partial x^{q^{\prime}}}{\partial \xi^{s}}\right) \mathcal{F}^{j^{\prime}}\right] \\
& =\frac{1}{2}\left\{\epsilon_{j^{\prime} p^{\prime} q^{\prime}} \epsilon^{i r s}\left[\frac{\partial}{\partial \xi^{i}}\left(\frac{\partial x^{p^{\prime}} \partial x^{q^{\prime}}}{\partial \xi^{r}} \frac{\boldsymbol{F}^{\prime}}{\partial \xi^{s}}\right) \mathcal{F}^{j^{\prime}}+\left(\frac{\partial x^{p^{\prime}}}{\partial \xi^{r}} \frac{\partial x^{q^{\prime}}}{\partial \xi^{s}}\right) \frac{\partial \mathcal{F}^{j^{\prime}}}{\partial \xi^{i}}\right]\right\} \\
& =\frac{1}{2} \epsilon_{j^{\prime} p^{\prime} q^{\prime}} \epsilon^{i r s}\left(\frac{\partial x^{p^{\prime}}}{\partial \xi^{r}} \frac{\partial x^{q^{\prime}}}{\partial \xi^{s}}\right) \frac{\partial \mathcal{F}^{j^{\prime}}}{\partial \xi^{i}}
\end{aligned}
$$

Assim, na variação:

$$
\begin{aligned}
\delta S_{j^{\prime}}^{i} \frac{\partial \mathcal{F}^{j^{\prime}}}{\partial \xi^{i}}= & \frac{1}{2} \epsilon_{j^{\prime} p^{\prime} q^{\prime}} \epsilon^{i r s}\left[\frac{\partial\left(\delta x^{p^{\prime}}\right)}{\partial \xi^{r}} \frac{\partial x^{q^{\prime}}}{\partial \xi^{s}}+\frac{\partial x^{p^{\prime}}}{\partial \xi^{r}} \frac{\partial\left(\delta x^{q^{\prime}}\right)}{\partial \xi^{s}}\right] \frac{\partial \mathcal{F}^{j^{\prime}}}{\partial \xi^{i}}= \\
= & \frac{1}{2} \epsilon_{j^{\prime} p^{\prime} q^{\prime}} \epsilon^{i r s}\left\{\frac{\partial}{\partial \xi^{r}}\left[\delta\left(x^{p^{\prime}}\right) \frac{\partial x^{q^{\prime}}}{\partial \xi^{s}} \frac{\partial \mathcal{F}^{j^{\prime}}}{\partial \xi^{i}}\right]+\right. \\
& \left.+\frac{\partial}{\partial \xi^{s}}\left[\delta\left(x^{q^{\prime}}\right) \frac{\partial x^{p^{\prime}}}{\partial \xi^{r}} \frac{\partial \mathcal{F}^{j^{\prime}}}{\partial \xi^{i}}\right]\right\}
\end{aligned}
$$

Obtém-se:

$$
\delta S_{j^{\prime}}^{i} \frac{\partial \mathcal{F}^{j^{\prime}}}{\partial \xi^{i}}=\epsilon_{j^{\prime} p^{\prime} q^{\prime}} \epsilon^{i r s}\left\{\frac{\partial}{\partial \xi^{r}}\left[\delta\left(x^{p^{\prime}}\right) \frac{\partial x^{q^{\prime}}}{\partial \xi^{s}} \frac{\partial \mathcal{F}^{j^{\prime}}}{\partial \xi^{i}}\right]\right\}
$$


Mas,

$$
\delta x^{p^{\prime}}=\frac{\partial x^{p^{\prime}}}{\partial \xi^{k}} \delta \xi^{k}
$$

Portanto:

$$
\begin{aligned}
\delta S_{j^{\prime}}^{i} \frac{\partial \mathcal{F}^{j^{\prime}}}{\partial \xi^{i}} & =\epsilon_{j^{\prime} p^{\prime} q^{\prime}} \epsilon^{i r s} \frac{\partial}{\partial \xi^{r}}\left(\frac{\partial x^{p^{\prime}}}{\partial \xi^{k}} \frac{\partial x^{q^{\prime}}}{\partial \xi^{s}} \frac{\partial \mathcal{F}^{j^{\prime}}}{\partial \xi^{i}} \delta \xi^{k}\right)= \\
& =\frac{\partial}{\partial \xi^{r}}\left[\epsilon_{j^{\prime} p^{\prime} q^{\prime}} \epsilon^{i r s}\left(\beta_{k}^{p^{\prime}} \beta_{s}^{q^{\prime}} \frac{\partial \mathcal{F}^{j^{\prime}}}{\partial \xi^{i}} \delta \xi^{k}\right)\right]
\end{aligned}
$$

Lembrando que é possível escrever:

$$
\beta_{k}^{p^{\prime}} \beta_{s}^{q^{\prime}}=\frac{1}{2}\left(\beta_{k}^{p^{\prime}} \beta_{s}^{q^{\prime}}-\beta_{s}^{p^{\prime}} \beta_{k}^{q^{\prime}}\right)+\frac{1}{2}\left(\beta_{k}^{p^{\prime}} \beta_{s}^{q^{\prime}}+\beta_{s}^{p^{\prime}} \beta_{k}^{q^{\prime}}\right)
$$

E, ainda:

$$
\begin{aligned}
2 \epsilon^{j^{\prime} m^{\prime} n^{\prime}} \epsilon_{r t l} S_{j^{\prime}}^{r} & =\epsilon^{j^{\prime} m^{\prime} n^{\prime}} \epsilon_{j^{\prime} p^{\prime} q^{\prime}} \epsilon_{r t l} \epsilon^{r s i} \beta_{s}^{p^{\prime}} \beta_{i}^{q^{\prime}}= \\
& =\left(\delta_{p^{\prime}}^{m^{\prime}} \delta_{q^{\prime}}^{n^{\prime}}-\delta_{q^{\prime}}^{m^{\prime}} \delta_{p^{\prime}}^{n^{\prime}}\right)\left(\delta_{t}^{s} \delta_{l}^{i}-\delta_{t}^{i} \delta_{l}^{s}\right) \beta_{s}^{r^{\prime}} \beta_{i}^{q^{\prime}}= \\
& =\left(\delta_{p^{\prime}}^{m^{\prime}} \delta_{q^{\prime}}^{n^{\prime}} \delta_{l}^{s} \delta_{l}^{i}-\delta_{p^{\prime}}^{m^{\prime}} \delta_{q^{\prime}}^{n^{\prime}} \delta_{t}^{i} \delta_{l}^{s}-\delta_{q^{\prime}}^{m^{\prime}} \delta_{p^{\prime}}^{n^{\prime}} \delta_{t}^{s} \delta_{l}^{i}+\delta_{q^{\prime}}^{m^{\prime}} \delta_{p^{\prime}}^{n^{\prime}} \delta_{t}^{i} \delta_{l}^{s}\right) \beta_{s}^{p^{\prime}} \beta_{i}^{q^{\prime}}= \\
& =\beta_{t}^{m^{\prime}} \beta_{l}^{n^{\prime}}-\beta_{l}^{m^{\prime}} \beta_{t}^{n^{\prime}}-\beta_{t}^{n^{\prime}} \beta_{l}^{m^{\prime}}+\beta_{l}^{n^{\prime}} \beta_{t}^{m^{\prime}}= \\
& =2\left(\beta_{t}^{m^{\prime}} \beta_{l}^{n^{\prime}}-\beta_{l}^{m^{\prime}} \beta_{t}^{n^{\prime}}\right)
\end{aligned}
$$

Então, substituindo (B.9) em (B.8), obtém-se:

$$
\beta_{k}^{p^{\prime}} \beta_{s}^{q^{\prime}}=\frac{1}{2} \epsilon^{t^{\prime} p^{\prime} q^{\prime}} \epsilon_{n k s} S_{t^{\prime}}^{n}+\frac{1}{2}\left(\beta_{k}^{p^{\prime}} \beta_{s}^{q^{\prime}}+\beta_{s}^{p^{\prime}} \beta_{k}^{q^{\prime}}\right)
$$

Mas,

$$
S_{j^{\prime}}^{r}=\frac{1}{2} \epsilon_{j^{\prime} p^{\prime} q^{\prime}} \epsilon^{r s i} \beta_{s}^{p^{\prime}} \beta_{i}^{q^{\prime}}
$$

Portanto, a equação (B.7) resulta em:

$$
\begin{aligned}
\delta S_{j^{\prime}}^{i} \frac{\partial \mathcal{F}_{\alpha}^{j^{\prime}}}{\partial \xi^{i}} & =\frac{\partial}{\partial \xi^{r}}\left\{\epsilon_{j^{\prime} p^{\prime} q^{\prime}} \epsilon^{i r s}\left[\frac{1}{2} \epsilon^{t^{\prime} p^{\prime} q^{\prime}} \epsilon_{n k s} S_{t^{\prime}}^{n}+\frac{1}{2}\left(\beta_{k}^{p^{\prime}} \beta_{s}^{q^{\prime}}+\beta_{s}^{p^{\prime}} \beta_{k}^{q^{\prime}}\right)\right] \frac{\partial \mathcal{F}^{j^{\prime}}}{\partial \xi^{i}} \delta \xi^{k}\right\} \\
& =\frac{\partial}{\partial \xi^{r}}\left[\epsilon_{j^{\prime} p^{\prime} q^{\prime}} \epsilon^{i r s}\left(\frac{1}{2} \epsilon^{t^{\prime} p^{\prime} q^{\prime}} \epsilon_{n k s} S_{t^{\prime}}^{n}\right) \frac{\partial \mathcal{F}^{j^{\prime}}}{\partial \xi^{i}} \delta \xi^{k}\right] \\
& =\frac{\partial}{\partial \xi^{r}}\left[\epsilon_{j^{\prime} p^{\prime} q^{\prime}} \epsilon^{t^{\prime} p^{\prime} q^{\prime}} \epsilon^{i r s} \epsilon_{n k s} \frac{1}{2} S_{t^{\prime}}^{n} \frac{\partial \mathcal{F}_{\alpha}^{j^{\prime}}}{\partial \xi^{i}} \delta \xi^{k}\right]= \\
& =\frac{\partial}{\partial \xi^{r}}\left[2 \delta_{j^{\prime}}^{t^{\prime}} \epsilon^{s i r} \epsilon_{s n k} \frac{1}{2} S_{t^{\prime}}^{n} \frac{\partial \mathcal{F}_{\alpha}^{j^{\prime}}}{\partial \xi^{i}} \delta \xi^{k}\right]= \\
& =\frac{\partial}{\partial \xi^{r}}\left[\delta_{j^{\prime}}^{t^{\prime}}\left(\delta_{n}^{i} \delta_{k}^{r}-\delta_{k}^{i} \delta_{n}^{r}\right) S_{t^{\prime}}^{n} \frac{\partial \mathcal{F}_{\alpha}^{j^{\prime}}}{\partial \xi^{i}} \delta \xi^{k}\right]= \\
& =\frac{\partial}{\partial \xi}\left[\left(\delta_{n}^{i} \delta_{k}^{r}-\delta_{k}^{i} \delta_{n}^{r}\right) S_{j^{\prime}}^{n} \frac{\partial \mathcal{F}_{\alpha}^{j^{\prime}}}{\partial \xi^{i}} \delta \xi^{k}\right]
\end{aligned}
$$


Então:

$$
\delta S_{j^{\prime}}^{i} \frac{\partial \mathcal{F}_{\alpha}^{j^{\prime}}}{\partial \xi^{i}}=\frac{\partial}{\partial \xi^{r}}\left[S_{j^{\prime}}^{i} \frac{\partial \mathcal{F}_{\alpha}^{\gamma^{\prime}}}{\partial \xi^{i}} \delta \xi^{r}-S_{j^{\prime}}^{r} \frac{\partial \mathcal{F}_{\alpha}^{j^{\prime}}}{\partial \xi^{i}} \delta \xi^{i}\right]
$$

Observe que o termo cancelado na última equação corresponde simplesmente às equações de Euler em estado estacionário e, portanto, são nulas. Assim, finalmente, obtém-se:

$$
\delta S_{j^{\prime}}^{i} \frac{\partial \mathcal{F}_{\alpha}^{j^{\prime}}}{\partial \xi^{i}}=-\frac{\partial}{\partial \xi^{r}}\left(S_{j^{\prime}}^{r} \frac{\partial \mathcal{F}_{\alpha}^{j^{\prime}}}{\partial \xi^{i}} \delta \xi^{i}\right)
$$




\section{APÊNDICE C - DEFINIÇÃO DOS COEFICIENTES NA FormulaÇÃo Característica das EquaÇÕES Adjuntas}

A tabela C.1 a seguir mostra os valores atribuídos aos coeficientes $C_{i j k}$ do sistema (4.15), que representa a formulação característica adjunta baseada nas equações de Euler bidimensionais. Os índices dos coeficientes são determinados da seguinte maneira: o primeiro indica a equação do sistema característico; o segundo, refere-se à variável adjunta a que está relacionado; e o último, ao tipo de derivada parcial. Assim, $C_{23 x}$, por exemplo, é o coeficiente do termo $\frac{\partial \psi_{3}}{\partial x}$ da segunda equação do sistema (4.15).

Vale ressaltar que, no caso, todos os coeficientes são constantes no tempo durante a solução adjunta para cada elemento da malha, uma vez que são formados por expressões matemáticas que envolvem apenas propriedades do escoamento (lembrando que a solução adjunta é determinada a partir de uma solução estacionária do escoemanto). Entretanto, eles variam de elemento para elemento. A definição destes coeficientes tem como finalidade facilitar a vizualização do sistema característico (que possuí termos bastante complicados) como um todo, mostrando que apesar de sua aparência "assustadora", ele nada mais é do que um sistema linear, que pode ter sua implementação numérica facilitada ao definir os termos $C_{i j k}$ propostos na tabela C.1. 


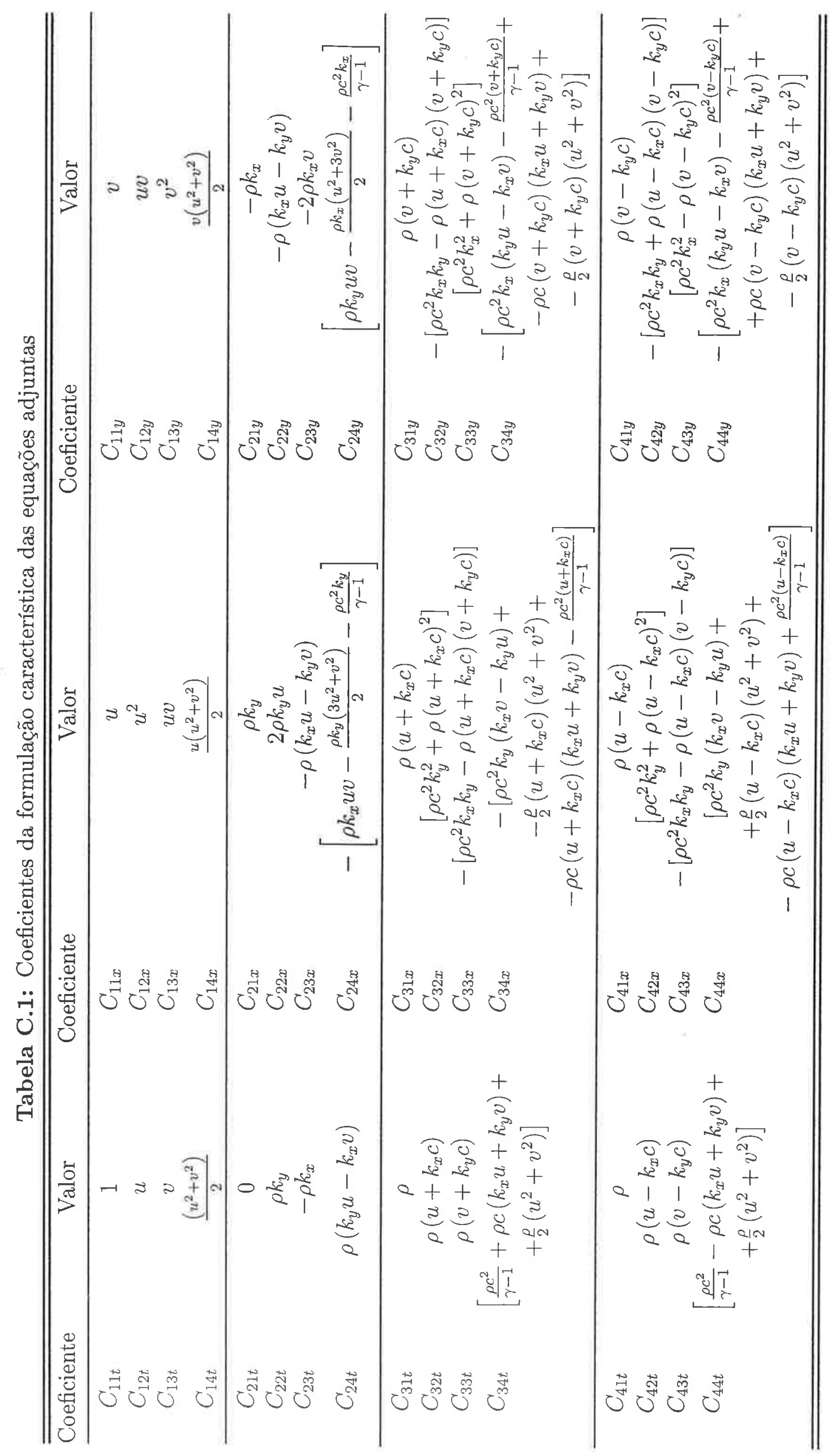

